\title{
miRNAs in protection and regeneration of dopaminergic midbrain neurons
}

\author{
Dissertation \\ in partial fulfillment for the award of the degree \\ "Doctor rerum naturalium" \\ of the Georg-August-University of Göttingen
}

within the doctoral program "Molecular Physiology of the Brain"

of the "Georg-August-University School of Science (GAUSS)"

submitted by

Anna-Elisa Roser

born in

Langenhagen (Germany) 


\section{Thesis Committee}

Prof. Dr. Paul Lingor (Department of Neurology, University Medical Center Göttingen)

Prof. Dr. André Fischer (Department of Psychiatry and Psychotherapy, University Medical Center Göttingen)

Prof. Dr. Tiago Fleming Outeiro (NeuroDegeneration and Restaurative Research, University Medical Center Göttingen)

\section{Members of the Examination Board}

$1^{\text {st }}$ Referee: Prof. Dr. Paul Lingor (Department of Neurology, University Medical Center Göttingen)

$2^{\text {nd }}$ Referee: Prof. Dr. André Fischer (Department of Psychiatry and Psychotherapy, University Medical Center Göttingen)

Prof. Dr. Tiago Fleming Outeiro (NeuroDegeneration and Restaurative Research, University Medical Center Göttingen)

Prof. Dr. Silvio Rizzoli (Department of Neuro- and Sensory Physiology, University Medical Center Göttingen)

Prof. Dr. Michael Hörner (Department of Cellular Neurobiology, Johann-FriedrichBlumenbach Institut, Georg-August-University Göttingen)

Dr. Sebastian Kügler (Department of Neurology, University Medical Center Göttingen) 



\section{Declaration}

Hereby I declare that the thesis entitled:

"miRNAs in protection and regeneration of dopaminergic midbrain neurons"

has been written independently and with no other sources and aids than quoted.

Göttingen, February 2016

Anna-Elisa Roser 

Meinen Eltern

Unsere Wünsche sind Vorgefühle der Fähigkeiten, die in uns liegen, Vorboten desjenigen, was wir zu leisten im Stande sein werden.

Johann Wolfgang von Goethe (1749-1832) 



\section{Table of contents}

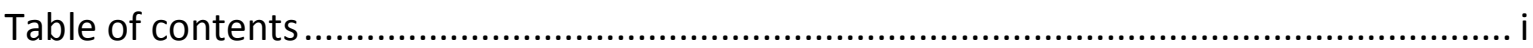

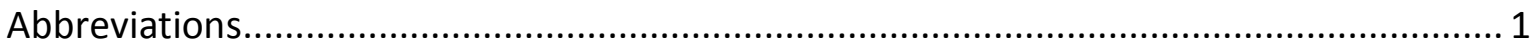

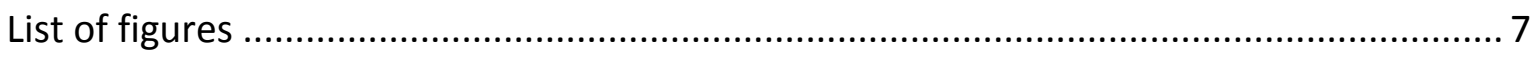

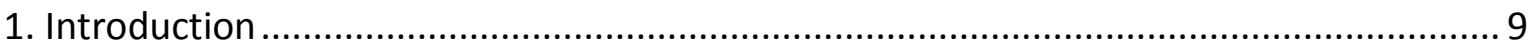

1.1 Neurodegenerative diseases - The costs of an aging society ............................... 9

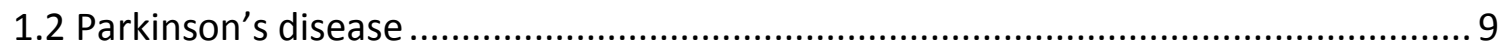

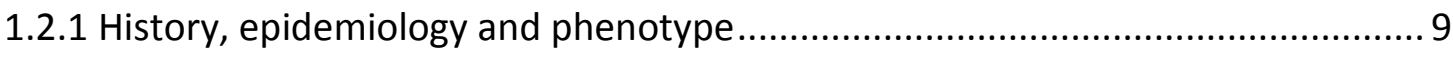

1.2.2 Disease progression and pathophysiology..................................................... 10

1.2.3 Etiology and pathogenesis: Possible causes for PD ......................................... 12

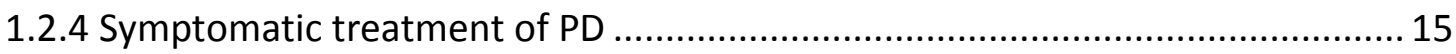

1.2.5 The need for regenerative or neuroprotective therapies - Approaches and

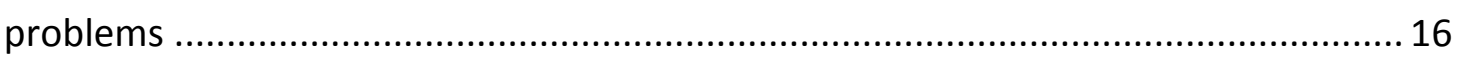

1.3 miRNAs - Biogenesis, function and role in neurodegenerative diseases ................. 19

1.3.1 Discovery, biogenesis and function of miRNAs............................................. 19

1.3.2 The role of miRNAs in neurodegenerative diseases ........................................ 22

1.3.3 The role of miRNAs in dopaminergic neuron biology .................................... 24

1.3.4 The potential of miRNAs providing neuronal protection and regeneration ...... 26

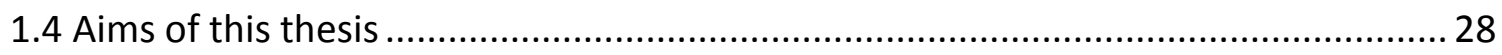

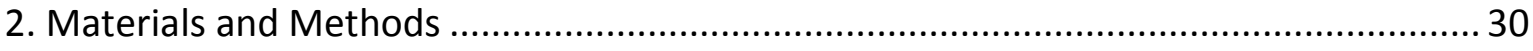

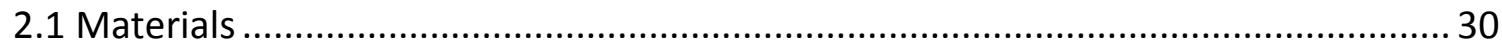

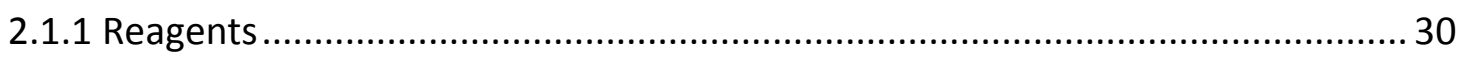

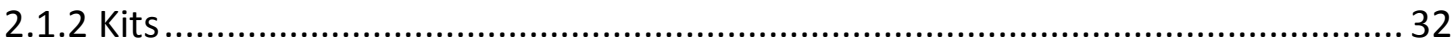

2.1.3 Buffers, solutions and cell culture medium ................................................. 32

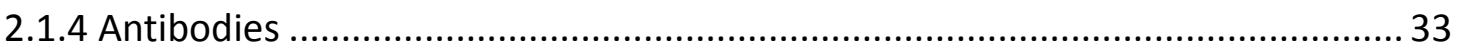

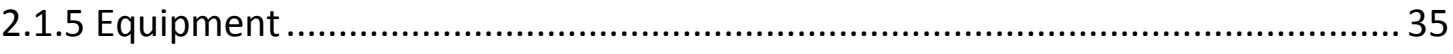

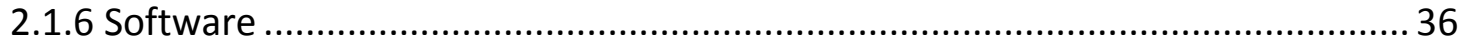

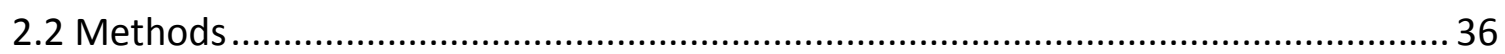

2.2.1 Animal surgery, injections and tissue processing ............................................ 36

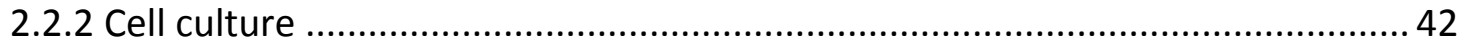


2.2.3 Molecular biology and biochemical techniques

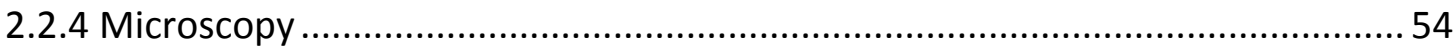

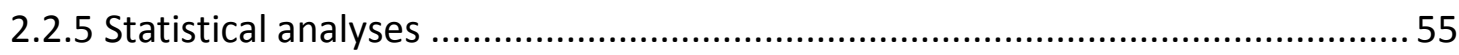

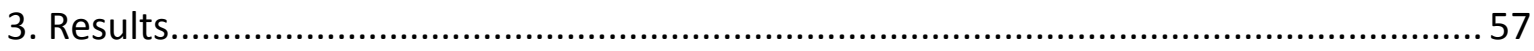

3.1 miRNAs in maturation and neurite outgrowth of primary midbrain neurons (PMN) -

Providing tools for neuronal protection and regeneration?

3.1.1 miRNA expression levels in developing PMNs - Major changes occur during

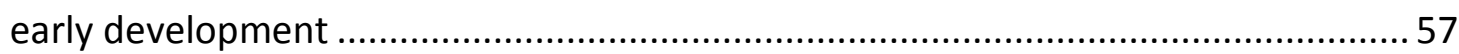

3.1.2 GDNF treatment lead to specific changes in the miRNAome of PMNs - Only miR-182 and miR-183 are significantly regulated....

3.1.3 Functional annotation of miR-182 and miR-183 target genes - Potential role in dopaminergic neuron biology

3.2 miRNA expression changes upon dopaminergic neuron degeneration and regeneration - Insights from the 6-OHDA mouse model for PD .................................66 66

3.2.1 The miRNAome of the murine SNpc containing midbrain................................66

3.2.2 Changes in miRNA expression of the murine midbrain upon degeneration of the

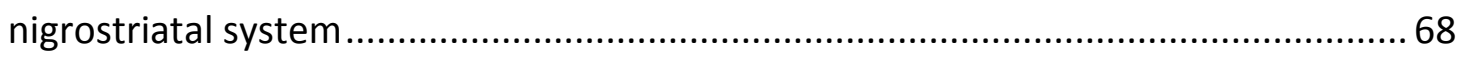

3.2.3 miRNAs involved in spontaneous regeneration of SNpc dopaminergic neurons after 6-OHDA lesion

3.3 Transfection of GDNF-regulated miR-182 and miR-183 - Beneficial effects on dopaminergic PMNs in vitro?

3.3.1 Increase in miR-182 and miR-183 leads to longer neurites in dopaminergic PMNs

3.3.2 Increased miR-182 and miR-183 protects dopaminergic PMNs from MPP+induced neurotoxicity

3.3.3 Increased miR-182 and miR-183 levels improve neurite regeneration in dopaminergic PMN.

3.4 Neurite outgrowth, neuroprotection and neurite regeneration - Mechanisms underlying the beneficial effects of miR-182 and miR-183 increase in dopaminergic PMNs

3.4.1 Increased neurite growth, regeneration and neuronal survival are not an effect of miRNA increase per se

3.4.2 Increased miR-182 and miR-183 levels lead to a reduction in Foxo3 and Foxo1 protein expression.

3.4.3 Effects of increased miR-182 and miR-183 on survival and growth associated signaling pathways 
3.5 Effects of increased miR-182 and miR-183 levels in the acute MPTP mouse model for PD .

3.5.1 Stereotactical injection of fluorescently labeled NC siRNA leads to transfection of dopaminergic neurons in the murine SNpc

3.5.2 Lipid-based transfection reagent does not lead to increased microglial invasion in the murine SNpc....

3.5.3 Injection of miR-182 and miR-183 mimics into the murine SNpc leads to a stable increase in miR-182 and miR-183 levels detected by qRT-PCR .

3.5.4 Increased miR-182 levels lead to a higher survival of dopaminergic neurons in the SNpc of mice after MPTP intoxication

3.5.5 Increased nigral miR-182 levels lead to attenuated dopaminergic fiber loss and increased dopamine levels in the striatum of MPTP-intoxicated mice 95

3.5.6 Increased miR-182 and miR-183 levels lead to a partial restoration of motor deficits 98

4. Discussion 102

4.1 miRNAs in maturation and neurite outgrowth of PMNs 102

4.1.1 miRNA expression in developing PMNs - major changes occur during early development 103

4.1.2 The regulated miRNAs are important for neuronal differentiation and function

4.1.3 GDNF treatment leads to a specific regulation of miR-182 and miR-183 expression 105

4.2 miRNA expression changes in the degenerating and regenerating murine SN ...... 107

4.2.1 miRNA expression changes in the degeneration model 108

4.2.2 miRNAs involved in spontaneous recovery of SNpc dopaminergic neurons after

6-OHDA lesion 110

4.3 Effects of increased miR-182 and miR-183 levels on dopaminergic PMNs........

4.3.1 Increased miR-182 and miR-183 levels lead to longer neurites and increased neurite regeneration in dopaminergic PMNs

4.3.2 Increased miR-182 and miR-183 levels protect dopaminergic PMNs from MPP ${ }^{+}$ induced degeneration

4.4 Effects of increased miR-182 and miR-183 levels in the MPTP mouse model for PD

4.4.1 Increased levels of miR-182 levels lead to protection of the nigrostriatal system in MPTP-intoxicated mice and partially rescues behavioral deficits ........................ 118

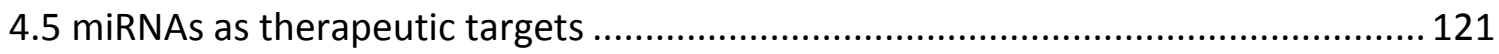


5. Concluding remarks. 123

6. Summary..... 124

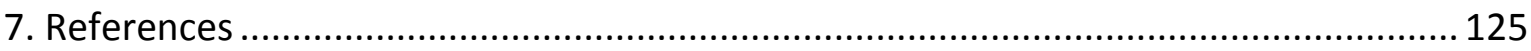

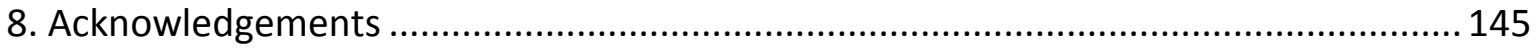

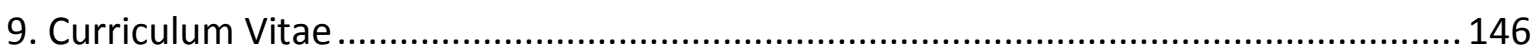




\section{Abbreviations}

3'UTR: three prime untranslated region

6-OHDA: 6-hydroxydopamine

AAV: adeno-associated virus

$A \beta$ : amyeloid $\beta$

AD: Alzheimer's disease

ALS: amyotrophic lateral sclerosis

AP: anterior-posterior

APS: ammonium peroxide sulfate

ATP: adenosine triphosphate

ATP13A2: ATPase type 13A2

BCL-2: B-cell lymphoma 2

BDNF: brain derived neurotrophic factor

BSA: bovine serum albumin

$\mathrm{CaCl}_{2}$ : calcium chloride

CCD: charge-coupled device

CDNF: cerebral dopaminergic neurotrophic factor

$\mathrm{C}_{6} \mathrm{H}_{8} \mathrm{O}_{7} * \mathrm{H}_{2} \mathrm{O}$ : citric acid

$\mathrm{CN}$ : centromedian nucleus

CNS: central nervous system

$\mathrm{CO}_{2}$ : carbon dioxide

CSF: cerebrospinal fluid

Cy: cyanine

DA: dopamine

DAB: 3,3'-diaminobenzidine 
DAPI: 4',6-diamidino-2-phenylindole

DAT: dopamine transporter

DAMP: damaged associated molecular pattern

DBS: deep brain stimulation

DIV: days in vitro

DNA: deoxyribonucleic acid

DOPAC: 3,4-dihydroxyphenylacetic acid

DPX: distrene-plastilizer-xylene

dsRNA: double-stranded RNA

DV: dorso-ventral

ECL: enhanced chemiluminescence

EDTA: ethylenediaminetetraacetic acid

Erk: extracellular-signal regulated kinase

ES: embryonic stem cells

fc: fold change

FCS: fetal calf serum

FGF20: fibroblast growth factor 20

GAPDH: Glycerinaldehyd-3-phosphate dehydrogenase

GDF5: growth / differentiation factor 5

GDNF: glial cell-line derived neurotrophic factor

GPe: external globus pallidus

GPi: internal globus pallidus

HBSS: Hank's balanced salt solution

$\mathrm{H}_{3} \mathrm{BO}_{3}$ : boric acid

$\mathrm{HCl}$ : hydrochloric acid

$\mathrm{HClO}_{4}$ : perchloric acid 
$\mathrm{H}_{2} \mathrm{C}_{6} \mathrm{O}$ : ethanol

HD: Huntington's disease

HDAC: NAD-dependent histone deacytelase

HEPES: 2-[4-(2-hydroxyethyl) piperazin-1-yl] ethanesulfonic acid

HiP: HiPerfect tranfection reagent

$\mathrm{HNO}_{3}$ : nitric acid

$\mathrm{H}_{2} \mathrm{O}$ : water

$\mathrm{H}_{2} \mathrm{O}_{2}$ : hydrogen peroxide

HPLC: high performance liquid chromatography

HRP: horseradish peroxidase

HVA: homovanillic acid

Iba1: ionized calcium binding adapter molecule 1

ICC: immunocytochemistry

IHC: immunohistochemistry

IPS: induced pluripotent stem cells

L-AA: L-ascorbic acid

LB: Lewy body

LRRK2: leucine-rich repeat kinase 2

MANF: mesencephalic astrocyte-derived neurotrophic factor

Mapk: mitogen-associated protein kinase

meCP2: methyl CpG binding protein 2

miR: MiRNA

miRNA: microRNA

ML: medio-lateral

MAOB: monoamine oxidase $B$

$\mathrm{MPP}^{+}$: 1-methyl-4-phenylpyridinium ion 
MPTP: 1-methyl-4-phenyl-1,2,3,6-tetrahydropyridine

mRNA: messenger RNA

$\mathrm{Na}_{2} \mathrm{~B}_{4} \mathrm{O}_{7}$ : sodium tetraborate

$\mathrm{NaCl}$ : sodium chloride

$\mathrm{NaHCO}_{3}$ : sodium hydrogen carbonate

$\mathrm{Na}_{2} \mathrm{HPO}_{4}$ : sodium hydrogen phosphate

$\mathrm{NaN}_{3}$ : sodium azide

$\mathrm{NaO}_{2} \mathrm{C}_{2} \mathrm{H}_{3}$ : sodium acetate

$\mathrm{NaOH}$ : sodium hydroxide

ncRNA: non-coding RNA

NC siRNA: negative control siRNA

NDD: neurodegenerative disorder

NGF: nerve growth factor

NGS: normal goat serum

$\mathrm{NH}_{4} \mathrm{Cl}$ : ammonium chloride

nt: nucleotides

OFT: open field test

ON: over night

PAMP: pathogen associated molecular pattern

PBS: phosphate buffered saline

PCR: polymerase chain reaction

PD: Parkinson's disease

PFA: paraformaldehyde

PI3K: phosphoinositide-3-kinase

piRNA: piwi-interacting RNA

PLO: poly-L-ornithin 
PMN: primary midbrain neurons

PPN: pedunculopontine nucleus

PNS: peripheral nervous system

qPCR: quantitative PCR

RISC: RNA-induced silencing complex

RNA: ribonucleic acid

ROCK: Rho kinase

ROS: reactive oxygen species

rpm: rounds per minute

rRNA: ribosomal RNA

$\mathrm{RT}$ : room temperature

SDS: sodium dodecyl sulfate

SDS-page: SDS-polyacrylamide gel electrophoresis

SEM: standard error of the mean

shRNA: small hairpin RNA

siRNA: small interfering RNA

snoRNA: small nucleolar RNA

SNP: single nucleotide polymorphism

SNpc: substantia nigra pars compacta

SNpr/SNr: substantia nigra pars reticulate

Stat3: signal transducer and activator of transcription 3

STN: subthalamic nucleus

Taok1: TAO-protein kinase 1

TBS: Tris-buffered saline

TBS-T: TBS-Tween20

TEMED: tetramethylethylenediamine 
TH: tyrosine hydroxylase

Tris: 2-Amino-2-hydroxymethyl-propane-1,3-diol

tRNA: transfer RNA

TTX: tetrodotoxin

UPS: ubiquitin-proteasome system

VA/VL: thalamus

VEEV: Venezuelan equine encephalitis virus

VTA: ventral tegmental area 


\section{List of figures}

Figure 1.2/1: Diagram of the basal ganglia circuits in (a) healthy subjects and (b) Parkinson's disease.

Figure 1.3/1: The canonical miRNA biogenesis pathway. 21

Figure 1.3/2: The non-canonical miRNA biogenesis pathway. 22

Figure 2.2/1: Illustration of the injection sites for stereotactic injection. 38

Figure 3.1/1: Small RNA sequencing reveals changes in the miRNAome of PMNs during maturation.

Figure 3.1/2: Massive regulation of miRNA expression during maturation of PMNs and functional analysis of regulated miRNAs.

Figure 3.1/3: GDNF treatment of PMNs leads to a specific upregulation of miR-182 and miR-183.

Figure 3.1/4: Functional analysis of miR-182 (left panel) and miR-183 (right panel) target genes (TargetScan mouse 6.2).

Figure 3.2/1: Small RNA sequencing of the midbrain in the 6-OHDA mouse model for PD.

Figure 3.2/2: Significantly regulated miRNAs in the murine midbrain 4 weeks (degeneration) and 12 weeks (regeneration) after striatal 6-OHDA lesion and functional analysis of their target genes.

Figure 3.3/1: Increased levels of miR-182 and miR-183 result in longer neurites in dopaminergic neurons in PMN cultures.

Figure 3.3/2: Increase in miR-182 and miR-183 leads to a higher survival of dopaminergic $\mathrm{PMNs}$ after $\mathrm{MPP}^{+}$treatment.

Figure 3.3/3: miR-182 and miR-183 transfection results in increased regeneration of $\mathrm{TH}+$ neurites after mechanical scratch lesion.

Figure 3.4/1: Effects on neuronal survival and neurite growth and regeneration are not caused by miRNA overexpression per se.

Figure 3.4/2: Increased levels of miR-182 and miR-183 lead to a decrease in Foxo protein levels.

Figure 3.4/3: Increased miR-182 and miR-183 lead to increased phosphorylation of Akt. 
Figure 3.4/4: Higher miR-182 and miR-183 levels lead to trend of increased Mapk (Erk) signaling.

Figure 3.4/5: Effect of miR-182 and miR-183 increase on Bcl2, Stat3 and phospho-S6 protein levels.

Figure 3.5/1: Transfection of SN dopaminergic neurons of mice with fluorescently labeled NC siRNA in vivo.

Figure 3.5/2: Transfection using a lipid-based transfection reagent is not increasing microglial invasion around the injection site.

Figure 3.5/3: Increase in miRNA levels is stable several days after injection of synthetic miRNA mimics into the murine $\mathrm{SN}$ in vivo.

Figure 3.5/4: Increase in miR-182 levels leads to an increase in survival of dopaminergic $\mathrm{TH}+$ neurons in the MPTP mouse model.

Figure 3.5/5: Increase in miR-182 levels leads to a higher dopaminergic striatal fiber density in the MPTP mouse model. 96

Figure 3.5/6: miR-182 increase leads to higher levels of striatal dopamine (DA) in the MPTP mouse model.

Figure 3.5/7: Effect of increased miR-182 and miR-183 levels on motor behavior in the MPTP mouse model. 99

Figure 4.3/1: Proposed model for GDNF and miR-182/miR-183 interactions and their effects on dopaminergic neurons. 116 
1. Introduction

\section{Introduction}

\subsection{Neurodegenerative diseases - The costs of an aging society}

Characteristic for neurodegenerative diseases is the progressive degeneration of neurons in the central or peripheral nervous system (CNS, PNS), resulting in a reduced innervation of target structures and leading to the loss of sensory, motor and/or higher cognitive functions, which causes progressive disability in affected persons. The most common neurodegenerative disorders are Alzheimer's disease (AD) and Parkinson's disease (PD), which mostly occur sporadically, but can also be inherited. In both diseases the neurodegeneration is caused by multifactorial mechanisms, e.g. protein misfolding and aggregation as well as mitochondrial dysfunction and activation of programmed cell death pathways (Bredesen et al. 2006; Skovronsky et al. 2006). Increasing life expectancy in the industrialized countries resulted in a higher incidence and prevalence of neurodegenerative disorders. The exact pathomechanisms remain unclear and thus, no curative therapies exist. The socioeconomic costs of neurodegenerative diseases are high and increase with disease progression (Skovronsky et al. 2006), thus the investigation of underlying pathomechanisms and the development of early diagnostic tools as well as therapeutic strategies that prevent further neurodegeneration and stop disease progression are of major importance.

\subsection{Parkinson's disease}

\subsubsection{History, epidemiology and phenotype}

The first scientific description of PD was published in 1817 as "An essay on the shaking palsy" by the British physician James Parkinson. In this essay he described patients that showed the core motor clinical features of the disease, which was later named after him, including rigidity, postural instability, resting tremor and bradykinesia. The degeneration of dopaminergic neurons in the substantia nigra pars compacta (SNpc) was confirmed in 1919 by Tretiakoff (Lees et al. 2008) and in 1959 the hypothesis arose that PD symptoms are caused by a lack of the neurotransmitter dopamine (Lees 2007). 
Lewy bodies were described in 1912 by Frederic Lewy, but it took another 85 years till the discovery of their major component, aggregated alpha-synuclein (Spillantini et al. 1997).

$P D$ is the second most frequent neurodegenerative disease after Alzheimer's disease. In industrialized countries its prevalence is about $0.3 \%$ of the total population. As PD is an age-related disorder that has an average disease onset at around 60 years, its prevalence increases to $1-2 \%$ in people older than 60 years of age (de Lau \& Breteler 2006; Dexter \& Jenner 2013) and to $4 \%$ in people older than 85 (Nuytemans et al. 2010). Men are more often affected than women.

Approximately $5 \%$ of all PD cases are inherited (familial PD) and caused by mutations in different genes, but duplications and triplications of alpha-synuclein have also been described; so far 17 autosomal dominant and autosomal recessive mutations have been identified (Dexter \& Jenner 2013). However, the majority of PD cases occur sporadically without identifiable cause (idiopathic or sporadic PD). Nevertheless, many of the mechanisms underlying familial PD are also found in sporadic PD, e.g. oxidative stress, mitochondrial dysfunction and protein misfolding (Dexter \& Jenner 2013).

The clinical diagnosis of PD is still strongly dependent on impaired motor functions. Diagnosis is established when the motor symptoms start to occur, which is comparably late in pathophysiological disease progression. The motor symptoms of PD include rigidity, postural instability, bradykinesia, resting tremor, flexed posture, freezing, mask-like facial expression and problems to speak and swallow (Jankovic 2008). In addition to these, also non-motor symptoms like autonomic dysfunction, olfactory dysfunction (hyposmia), sleep disorders and psychiatric problems, like dementia and depression, are found in PD (Jankovic 2008; Smith et al. 2012). Non-motor symptoms were a long time not recognized as symptoms of PD but are getting more into focus nowadays. For instance, rapid eye movement sleep behavior disorder and hyposmia are discussed as early symptoms or pre-parkinsonian states (Borek et al. 2007; Ponsen et al. 2004).

\subsubsection{Disease progression and pathophysiology}

One of the major shortcomings in PD treatment is its late diagnosis, as the clinical manifestation of the disease occurs late in disease progression. Even though the classical 
hallmark of PD is the degeneration of dopaminergic neurons in the SNpc that is responsible for the classical motor symptoms, the intraneuronal pathology starts in the medulla oblongata and spreads over the brain stem and the basal ganglia to the neocortex, explaining the occurrence of non-motor symptoms and underlining that PD is a systemic disorder (Braak et al. 2003; Pereira et al. 2012). Nevertheless, dopaminergic neurons in the SNpc are particularly prone to neurodegeneration and their loss leads to the progressive motor symptoms characteristically for PD. At time of clinical manifestation, the striatal loss of dopaminerigc axon terminals is stronger than the degeneration of nigral cell bodies, which leads to the assumption that degeneration starts in the terminals leading to axonal degeneration and in its progression to neuronal cell death (Burke \& O'Malley 2013; Tönges et al. 2012).

The neuromelanin-containing dopaminergic neurons in the SNpc are part of the basal ganglia and thus involved in the regulation of voluntary movements. Their long unmyelinated axons project into the striatum where the dopaminergic terminals provide input to the spiny striatal neurons and modulate their activity by targeting the dopamine receptors $D_{1}$ and $D_{2}$. Excitatory input is mediated by activation of D1 receptors on the GABAergic spiny cells that target the internal globus pallidus (GPi) directly, therefore called direct pathway, leading to its inhibition and an excitation of the thalamus and the motor cortex. D2 receptors on spiny neurons mediate inhibitory input to the external globus pallidus (GPe) which in turn lead to less excitatory outputs of the subthalamic nucleus that projects to the GPi and activates it via the indirect pathway. The GPi projects to the ventral nucleus of the thalamus which in turn has efferent projections to the motor cortex that controls motor behavior and among others provides input to the spinal cord, the striatum and the substantia nigra pars reticulata (SNpr) (Kandel et al. 2000). These complex circuits are crucial for voluntary movement generation and a disturbance in transmitter levels may lead to motor dysfunctions. Degeneration of dopaminergic terminals leads to a depletion of striatal dopamine and therefore to an increased activation of the GPi that in turn leads to a decreased activity of the thalamus and motor cortex (summarized in figure 1.2/1) (Obeso et al. 2000; Smith et al. 2012). 


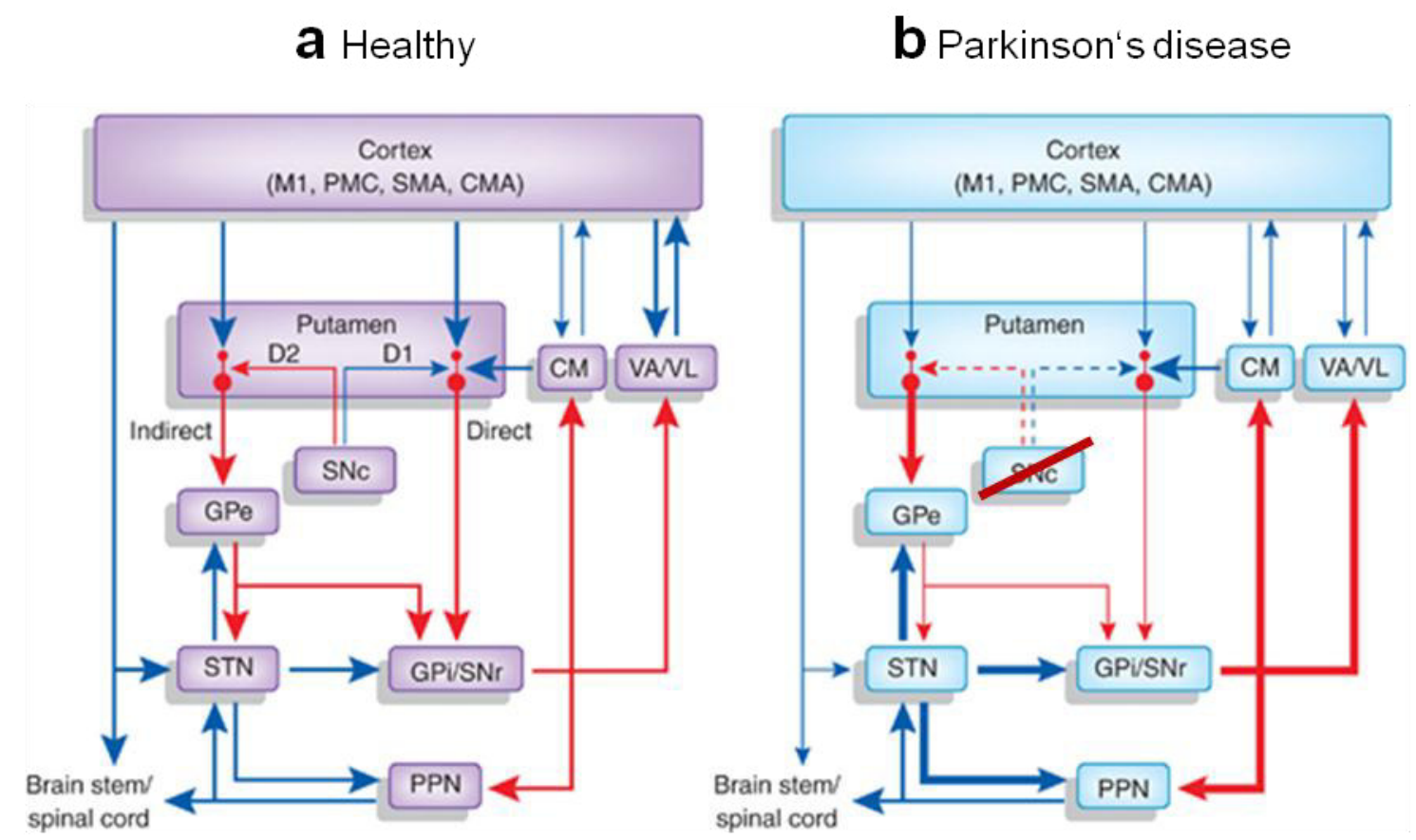

Figure 1.2/1: Diagram of the basal ganglia circuits in (a) healthy subjects and (b) Parkinson's disease. The thickness of arrows indicates signal strength. Blue arrows represent activating input; read arrows indicate inhibiting input. Loss of SNpc signals in PD results in less cortical activation and thus to the cardinal motor symptoms in PD (GPe=external globus pallidus; GPi=internal globus pallidus; $\mathrm{STN}=$ subthalamic nucleus; $\mathrm{SNr}=$ substantia nigra reticulate; PPN=pedunculopontine nucleus; $C M=$ centromedian nucleus; VA/VL=thalamus). (Smith et al. 2012, modified)

\subsubsection{Etiology and pathogenesis: Possible causes for PD}

There are several genes known to be involved in the formation of the rare familial forms of PD. Among them are mutations of the alpha-synuclein gene, the E3 ligase Parkin, LRRK2, DJ-1, Pink1 and ATP13A2 (Dexter \& Jenner 2013). The causes leading to the formation of idiopathic PD are not fully understood. In recent years it has become clear that a variety of factors play a role, among them genetic predisposition and environmental risk factors (Schapira \& Jenner 2011). The cellular mechanisms underlying degeneration of SNpc dopaminergic neurons are extensively studied and the most attention was paid to protein mishandling and aggregation and mitochondrial dysfunction and oxidative stress. 
1. Introduction

\section{Protein mishandling and aggregation}

One major hallmark of PD are cytoplasmatic inclusions, so called Lewy bodies (LBs), that are found in dopaminergic neurons as well as other affected brain areas in PD patients (Braak et al. 2003; Forman et al. 2004). LBs consist mainly of aggregated alphasynuclein, neurofilaments, and ubiquitin (Spillantini et al. 1997). The role of LBs in the development of PD is still under debate and it is not clear whether they are detrimental and lead to neuronal cell death (Braak et al. 2003; Braak et al. 2003; Lu et al. 2005), or if they have protective properties by sequestering toxic material from the cytoplasm (Parkkinen et al. 2011; Tompkins \& Hill 1997). Nevertheless, the presence of these large inclusion bodies in neurons of patients gives evidence that protein handling in affected neurons is malfunctioning. Another interesting point is the presence of alpha-synuclein in idiopathic PD cases. In familial PD, there are 5 alpha-synuclein mutations known till today (Proukakis et al. 2013), and together with polymorphisms of SNCA they lead to oligomerization, fibril formation and aggregation of alpha-synuclein (Lázaro et al. 2014). Interestingly, patients with idiopathic PD that do not have any alpha-synuclein mutation, show also aggregation and Lewy body formation, which again leads to the assumption that this is caused not only by excess protein but that malfunctioning protein degradation pathways are affected (Moore et al. 2003). A major player in protein degradation is the ubiqitin-proteasome system (UPS) which uses ubiqitin as a marker that targets cytosolic and nuclear proteins for proteolysis (Cooper 2000). Growing evidence suggests involvement of UPS malfunction in the formation of idiopathic and also familial PD (Giasson \& Lee 2003; Moore et al. 2003). Evidence ranges from mutations in parkin, a gene encoding for the E3 ligase Parkin that is responsible for substrate recruitment, in familial PD (Kitada et al. 1998), to deficits in the 20/26s proteasome in idiopathic PD patients (McNaught et al. 2002; McNaught et al. 2003) and occurrence of LB-like inclusions after UPS inhibition in rats (McNaught et al. 2004). Nevertheless, further investigations are necessary to explain the role of the UPS in PD.

\section{Mitochondrial dysfunction and oxidative stress}

Neurons are specifically dependent on mitochondrial function. They have a very high energy demand because of their high metabolic activity and their size and polarity requires an efficient mitochondrial transport system to sides of high energetic activity like 
synapses (Exner et al. 2012). Dopaminergic neurons in particular are highly susceptible for oxidative stress due to dopamine catabolism and require mitochondrial control systems. Thus, it is not surprising that mitochondrial dysfunction can lead to neurodegeneration. The idea that mitochondrial dysfunction could be involved in the formation of idiopathic PD was raised in the 1980's with the discovery of 1-methyl-4-phenyl-1,2,3,6tetrahydropyridine (MPTP). MPTP was a contaminant of illegal MPPP synthesis, a drug that was used intravenously and caused, if contaminated with MPTP, a parkinsonian syndrome. MPTP can cross the blood-brain-barrier and is then metabolized within glial cells to its active component $\mathrm{MPP}^{+}$. $\mathrm{MPP}^{+}$is transported into dopaminergic neurons via the dopamine transporter (DAT), where it inhibits complex I of the mitochondrial respiratory chain (Gerlach et al. 1991; Przedborski \& Jackson-Lewis 1998). Even though the MPTP induced Parkinsonism is acute and toxin induced and therefore different from the slow progression of idiopathic PD, there are still shared features and till today complex I inhibitors (MPTP, 6-OHDA, rotenone) are used to model PD (Bezard \& Przedborski 2011). Also in favor of the involvement of mitochondrial dysfunction due to complex I inhibition are reports that complex I activity is strongly reduced in the SNpc of PD patients (Janetzky et al. 1994; Mann et al. 1994; Schapira et al. 1990). Inhibition of complex I leads to a decreased ATP production and decreases the production of reactive oxygen species (ROS), leading to energy failure and oxidative stress. Oxidative stress in turn leads to damage of the mitochondrial DNA and enzymes of the respiratory chain, triggering further ROS production (Henchcliffe \& Beal 2008; Lin \& Beal 2006). Another factor emphasizing the potential role of mitochondria in the formation of PD is that many of the genes involved in the formation of familial PD directly or indirectly involve mitochondria (e.g. alpha-synuclein, LRRK2, DJ-1, Pink1 and parkin) (Lin \& Beal 2006).

Dopaminergic neurons have per se a higher oxidative burden than other neurons, because they produce $\mathrm{H}_{2} \mathrm{O}_{2}$ during the recycling of dopamine, which can interact with transition metals and form hydroxyl radicals. Furthermore the spontaneous autoxidation of dopamine leads to the formation of toxic ROS and $\mathrm{H}_{2} \mathrm{O}_{2}$ (Sidhu et al. 2004).

Another factor suggested for excessive production of ROS in PD is the increased occurrence of transition metals. Especially elevated levels of free iron lead to an abnormal oxidation of dopamine to the toxic species 6-OHDA and dopamine-quinone. These species could induce dopaminergic neurotoxicity and further release of iron from the iron storage 
pool resulting in a vicious circle of mitochondrial impairment, oxidative stress and further imbalance in iron homeostasis (Jomova et al. 2010).

Oxidative stress could also be induced by non-neuronal cells. Infiltration of microglial cells into affected brain regions is characteristic for neurodegenerative diseases. In the healthy brain, microglia are evenly distributed and constantly active, scanning the parenchyma for pathogen- and damage-associated molecular patterns (PAMPs and DAMPs). If PAMPs or DAMPs are detected microglial cells infiltrate the respective tissue and change their activation status from 'surveillance' to 'phagocytotic' to remove cellular debris. Furthermore they release different cytokines and chemokines that could enhance the inflammatory response or induce neuroprotection (Hanisch \& Kettenmann 2007; Kettenmann et al. 2011; Schwartz et al. 2006). Among the factors released by microglial cells are also ROS (Hanisch \& Kettenmann 2007). Reactive microglia are abundant in the SNpc of PD patients as well as in patients with MPTP induced parkinsonism and contribute to dopaminergic neuron degeneration (Hirsch \& Hunot 2009; McGeer \& McGeer 2008). Interestingly, the post mortem tissues of MPTP users show a prolonged activation of reactive microglia, even years after the acute intoxication (Langston et al. 1999). Further evidence for the involvement of microglial released ROS in dopaminergic neuron degeneration is given by different in vivo and in vitro studies (Le et al. 2001; Liberatore et al. 1999; Wu et al. 2002).

As indicated, there is a complex interplay between mitochondrial function, glial activation, production of ROS and protein mishandling involved in dopaminergic neurodegeneration in PD, and until today neither the precise mechanisms nor the causes are fully understood.

\subsubsection{Symptomatic treatment of PD}

Due to the lack of knowledge regarding the cause of PD, there is currently no curative therapy available. Patients are provided with symptomatic treatment that should attenuate the symptoms of PD. Till today the gold-standard in PD treatment is the pharmacological supplementation of dopamine by oral application of its precursor levodopa (L-DOPA) combined with a substance that inhibits its peripheral conversion to 
dopamine (decarboxylase inhibitor). In milder cases without advanced motor symptoms monoamine oxidase $B(M O A B)$ inhibitors are given to diminish dopamine degradation after release (Connolly \& Lang 2014). Major shortcomings of the dopamine replacement therapy are at one hand adverse effects like motor complications (e.g. levodopa induced dyskinesia), compulsive behavior, nausea and hallucinations, and at the other hand the inefficient targeting of dopamine-independent non-motor symptoms (e.g. depression and cognitive impairment) (Connolly \& Lang 2014; Hurtig 1997). Additionally, after years of dopamine replacement therapy, usually symptoms arise that are not responsive to the drugs.

Alternatives to pharmacological treatment are rare; one possibility is deep brain stimulation (DBS). DBS requires the surgical implantation of electrodes into the subthalamic nucleus, the GPi or the thalamus. Via high frequency stimulation of the respective area PD symptoms are diminished (Benabid et al. 2009; Bronstein et al. 2011). Because of the surgical risks, DBS is only applied in rather advanced cases of PD.

\subsubsection{The need for regenerative or neuroprotective therapies - Approaches and problems}

As mentioned above, PD can be diagnosed with the onset of clinical motor symptoms. A problem is that the first motor symptoms occur late in disease progression when approximately $60 \%$ of the nigrostriatal projections are already degenerated (Jellinger 2012). By now, there is no reliable biomarker known that might predict disease onset. Thus, it is of major importance to develop treatment strategies that prevent further degeneration of remaining dopaminergic SNpc neurons or induce regeneration of the nigrostriatal system.

One major problem in the injured or diseased CNS is the prevention of neuronal regeneration by extracellular cues (e.g. glial responses) and a limited intrinsic regeneration capacity in adult neurons (Fitch \& Silver 2008). Nevertheless, there is evidence that also adult neurons have the ability to sprout and thus, regeneration of the adult CNS is in principle possible. In toxin-induced animal models for PD spontaneous regrowth of dopaminergic nigrostriatal projections could be observed in different species. 
In rats that received 6-OHDA injections in the SNpc vigorous axonal sprouting could be observed 4 months post-lesion (Finkelstein et al. 2000). In MPTP-intoxicated monkeys and mice, the extent of spontaneous regeneration was linked to the dimension of degeneration (Elsworth et al. 1999; Mitsumoto et al. 1998). These examples demonstrate that the adult dopaminergic nigrostriatal system in principle has the ability to regenerate after a lesion, but by now it is not known whether compensatory sprouting occurs in PD patients or how it could be facilitated or induced.

Recently, several approaches to induce regeneration of the nigrostriatal system or avoid further degeneration of SNpc neurons were proposed. Among them was extensive research on grafting of fetal dopaminergic neurons or embryonic/induced pluripotent stem cells (ES; IPS). Implantation of fetal dopaminergic neurons into toxin-induced animal models for PD led to improved motor symptoms and increased striatal innervation (Bankiewicz et al. 1990; Redmond et al. 1986). After these promising preclinical studies grafts of fetal dopaminergic neurons were also implanted into the brains of patients with PD. The grafts can survive in the brains of PD patients but the degree of symptomatic improvement is highly variable and strongly dependent on different factors. Additionally, severe side effects like dyskinesia can occur (Freed et al. 2001; Piccini et al. 2005). With the emergence of advanced stem cell technology transplantation of undifferentiated ES or IPS became a novel possibility in PD research. Stem cells are the optimal source for cell replacement therapy because they are self-renewing and multipotent. It has been shown that mouse ES transplanted to 6-OHDA lesioned rats can spontaneously differentiate into dopaminergic neurons (Bjorklund et al. 2002). Transplantation of neuronal precursor cells derived from monkey ES attenuated MPTP-induced neurological symptoms in monkeys (Takagi et al. 2005). As undifferentiated ES always bear the risk of tumor formation after transplantation there is the aim to differentiate dopaminergic neurons out of IPS. These cells can be sorted and might improve the safety of cell replacement therapies (Sundberg et al. 2013).

Another important approach to facilitate regeneration and provide neuroprotection of the nigrostriatal system is the application or expression of neurotrophic factors. Neurotrophic factors are important for the development and maintenance of neurons. Several neurotrophic factors have been investigated for their neuroprotective potential for dopaminergic neurons. The neurotrophins that have a 
selective effect on dopaminergic neurons are glial cell-line derived neurotrophic factor (GDNF), neurturin, growth / differentiation factor 5 (GDF5), mesencephalic astrocytederived neurotrophic factor (MANF) and cerebral dopaminergic neurotrophic factor (CDNF) (Sullivan \& Toulouse 2011). The most promising factor is GDNF, belonging to the GDNF family of ligands, which has been extensively studied in vivo and in vitro and was already tested in clinical trials. GDNF has been shown to promote the differentiation and survival of dopaminergic neurons in culture. Additionally, treatment with GDNF could prevent those neurons from degeneration upon 1-methyl-4-phenylpyridinium ion (MPP+) treatment in vitro (Hou et al. 1996; Krieglstein et al. 1995; Lin et al. 1993). Similar neuroprotection and functional effects could also been observed in different animal models of PD, consequently it acts also on the adult dopaminergic neurons (Cheng et al. 1998; Kearns \& Gash 1995; Kordower et al. 2000). The administration site and mode (transfusion of the recombinant protein or expression by viral vectors) are crucial for the effectiveness of GDNF delivery (Sullivan \& Toulouse 2011). After first successful trials of intrastriatal GDNF delivery in human PD patients (Patel et al. 2005; Slevin et al. 2005), another randomized placebo-controlled trial reported no relief of motor symptoms (Lang et al. 2006). In addition, safety issues were raised when antibodies against GDNF were found in approximately $10 \%$ of patients that received striatal GDNF infusion, which could lead to adverse effects (Tatarewicz et al. 2007). General consensus is that GDNF is a valuable treatment option, but needs further preclinical studies to overcome safety issues and develop sustainable effects.

Another possibility to achieve protection or regeneration of dopaminergic neurons is the pharmacological modulation of intrinsic signaling and disease relevant pathways. Potential candidates to prevent neurodegeneration are inhibition of apoptotic and necrotic cell death pathways as well as application of ROS scavengers and energy mimetics and inhibitors of alpha-synuclein toxicity (Dawson \& Dawson 2002). Furthermore modulation of kinase activity (e.g. Akt, JNK, LRRK2, MAPK, ROCK) is a promising treatment option for PD because there is evidence that inhibition or activation of certain kinases is preventing neurodegeneration in preclinical studies (Burke 2007; Deng et al. 2011; Saal et al. 2015; Tönges et al. 2012).

Despite all efforts, the development of an applicable tool to prevent dopaminergic neuron cell death and facilitate regeneration of the nigrostriatal system in PD was still not 
successful and new ideas for treatment strategies are urgently needed. It is known that endogenous microRNAs (miRNAs) exert different functions in the CNS. As they usually target not only one but multiple proteins and pathways, they might be powerful tools for understanding PD pathology and development of new treatment strategies.

\section{3 miRNAs - Biogenesis, function and role in neurodegenerative diseases}

\subsubsection{Discovery, biogenesis and function of miRNAs}

Approximately $1.5 \%$ of the human genome accounts for protein-coding sequences, the remaining genome is associated with introns, regulatory DNA sequences, retrotransposons and non-coding RNAs or by now without known function. Non-coding RNA genes produce regulatory RNA species that play an important role in translation, post-transcriptional regulation of gene expression and modification of RNA. By now different classes of non-coding RNAs have been revealed, among them ribosomal RNA (rRNA), transfer RNA (tRNA), long non-coding RNA (long ncRNA) and small ncRNAs (Eddy 2001). The group of small ncRNAs is comprised of different species, including small interfering RNA (siRNA), small nucleolar RNA (snoRNA), piwi-interacting RNA (piRNA) and miRNA (Stefani \& Slack 2008).

The first miRNA was discovered and described in Caenorhabditis elegans by Lee et al. in 1993. They found that lin-4, a gene known to be important in larval development, was not protein-coding but produced a pair of small RNAs. They also noticed that one of these RNAs has antisense complementarity to the 3'UTR of the lin-14 gene. The idea and discovery that this let-4 RNA might post-transcriptionally regulate the expression of the lin-14 protein was brought up and published by Arasu and colleagues (1991) and Wightman and colleagues (1993) from the Ruvkun lab. Since then a rising number of miRNAs has been discovered in animals, plants and some viruses and it became clear that they play a role in a variety of biological functions and might regulate the majority of protein-coding genes.

miRNAs are between 21 and 26 nucleotides (nt) long and transcribed from miRNA genes either individually or in clusters of 2-7 miRNAs, or are excised from the introns or 3 '-UTR of protein-coding genes or exons and introns from non-coding genes. In mammals 
miRNA genes are also found in repetitive regions, suggesting that transposable elements play a role in creation of new miRNA genes (Mendes et al. 2009).

miRNA biogenesis takes place in the nucleus (transcription and primary processing) and the cytoplasm (further processing and incorporation into RNA-induced silencing complex (RISC)). There are different ways how mature miRNAs can be generated, but the majority is derived from the so-called canonical pathway which is dependent on the RNase III enzymes Drosha and Dicer (Ghildiyal \& Zamore 2009). The miRNA gene is typically transcribed by the RNA Polymerase II (in some cases RNA polymerase III), resulting in a long primary transcript with hairpin structure, the so-called pri-miRNA. This pri-miRNA is cleaved at its base by the nuclear protein complex formed out of Drosha and the double stranded RNA (dsRNA) binding protein DGCR8, releasing a hairpin that is usually between 55 and 70 nt long, the pre-miRNA (Yang \& Lai 2011; Yeom et al. 2006). The pre-miRNA is exported to the cytoplasm by Exportin-5, which recognizes its $3^{\prime}$ overhangs. In the cytoplasm the helical turns of the hairpin are cleaved by Dicer, leading to the formation of an unstable $~ 22 \mathrm{nt}$ long miRNA duplex structure. The unwinding of the duplex structure usually happens in association with Ago proteins, which together with the mature miRNA form the RNA silencing effector RNA induced silencing complex (RISC) (Kim \& Kim 2012). RISC can post-transcriptionally regulate protein expression in different ways: When the miRNA:mRNA pair shows perfect complementarity it leads to degradation of the mRNA. When there is a not sufficient complementarity for cleavage this leads to translational repression or deadenylation (summarized in figure 1.3/1) (Fabian et al. 2010; Yang \& Lai 2011). 
1. Introduction

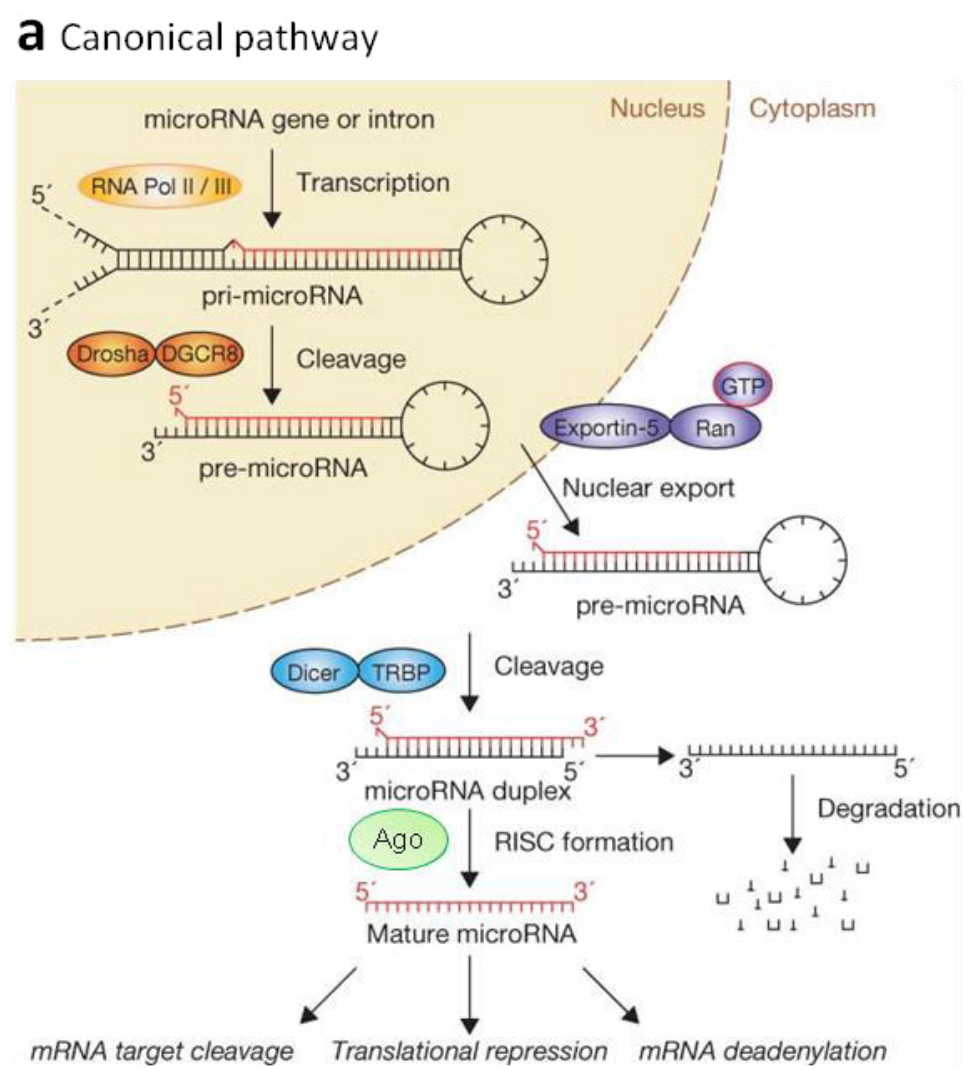

Figure 1.3/1: The canonical miRNA biogenesis pathway. miRNA genes are transcribed by RNA polymerase II (or III; RNA Pol II/III) and processed by Drosha/DGCR8. The pre-miRNA is exported to the cytoplasm where it is further processed and incorporated into Ago for RISC formation (Modified from Winter et al. 2009).

In addition, miRNAs can derive from different non-canonical pathways that are either Drosha/DGCR8-independent but Dicer-dependent or vice versa. Drosha/DGCR8 independent are pre-miRNAs deriving from "mirtrons" (RNA duplexes mapped to short introns), tRNAs, box H/ACA- and box C/D snoRNAs and endogenous shRNAs (summarized in figure $1.3 / 2$ a). An example for Dicer-independent miRNA biogenesis is miR-451; the pri-miR-451 is processed by Drosha/DGCR8 resulting in a pre-miRNA that is with $~ 18 \mathrm{nt}$ to short for Dicer processing. This pre-miR-451 is directly incorporated into Ago2 where it is sliced, resulting in a mature miR-451-RISC (see figure $1.3 / 2$ b) (Yang \& Lai 2011). Keeping in mind the fast progression of research in this field it would not be surprising if even more miRNA biogenesis pathways emerge in future studies.

Since their discovery substantial knowledge has been accumulated, giving evidence that miRNAs are involved in almost every biological process and developmental program. 
b Dicer-independent

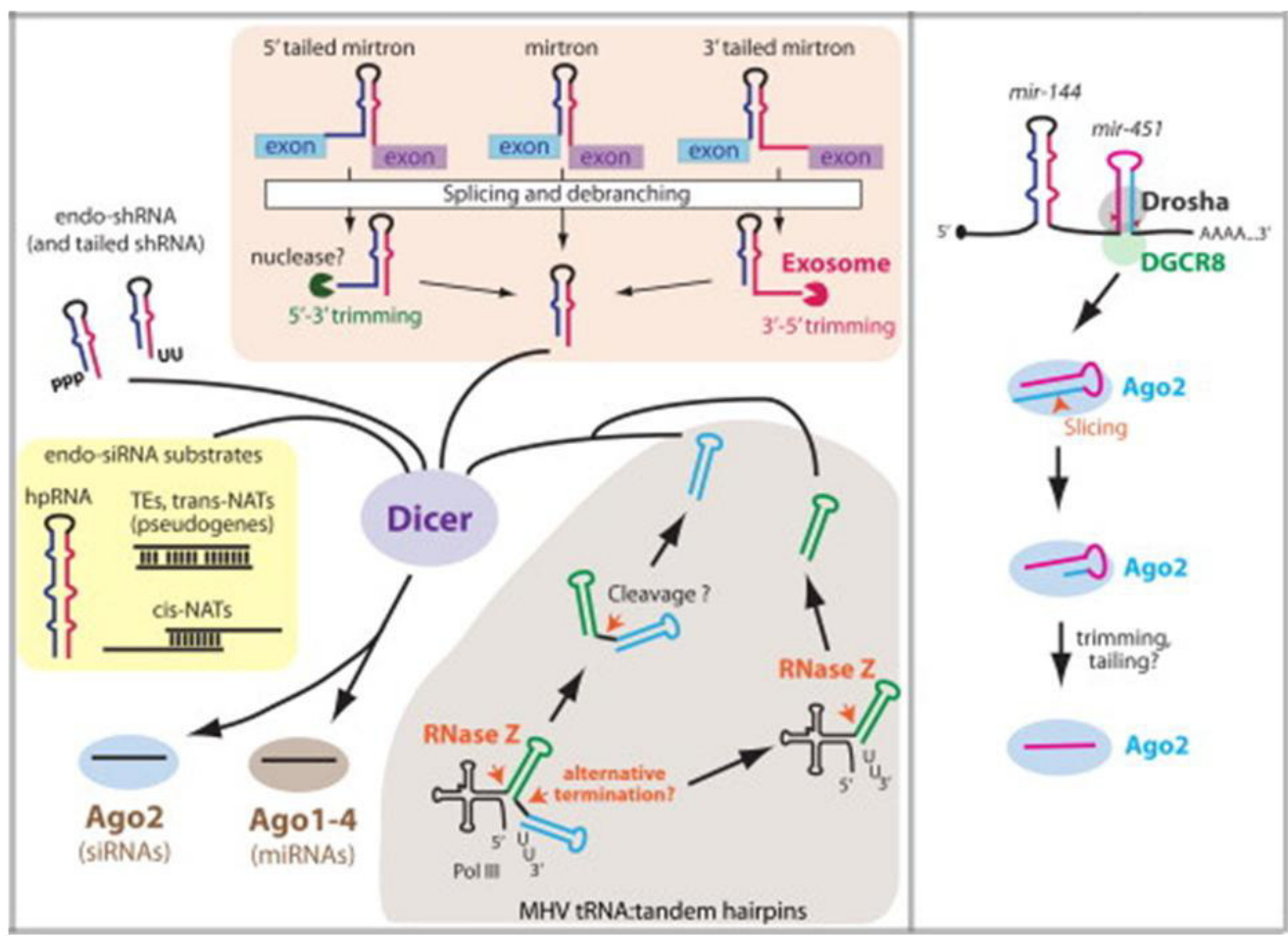

Figure 1.3/2: Non-canonical miRNA biogenesis pathways. (a) Drosha/DGCR8-independent miRNA biogenesis pathways. (b) Dicer-independent miRNA biogenesis (modified from Yang \& Lai 2011).

\subsubsection{The role of miRNAs in neurodegenerative diseases}

The importance of miRNA-mediated regulation of protein expression in nervous system development and maintenance is well-established (Kapsimali et al. 2007; Miska et al. 2004). Initial studies in animals lacking proteins of the miRNA biogenesis machinery (e.g. Dicer, DGCR8) showed that miRNAs are crucial for brain development and long-term neuronal integrity (Davis et al. 2008; Kim et al. 2007; Schaefer et al. 2007). In addition several brain specific miRNAs have been discovered; miR-124 for example counteracts REST and induces neuronal fate and is important for CNS development, miR-134 is important for dendritic spine formation and miR-132 is involved in neurite outgrowth (Magill et al. 2010; Makeyev et al. 2007; Schratt et al. 2006; Visvanathan et al. 2007).

Evidence that miRNA dysregulation occurs in neurodegenerative diseases was given by miRNA profiling studies of human brain tissue of patients and analysis of animal models. In Huntington's disease (HD) patients it was shown that miR-9 expression in the 
cerebral cortex is decreasing with disease progression. miR-9 is regulating REST, which is usually suppressing the expression of neuronal genes in non-neuronal cells. With decreasing miR-9 levels REST accumulates in the cytoplasm and translocates to the nucleus where it suppresses the expression of neuronal genes and thus might induce neurodegeneration (Packer et al. 2008). Another study showed that neuronal miR-132 is downregulated in the brains of HD patients and in a mouse model for HD (Johnson et al. 2008). Also pre-symptomatic HD patients show elevated levels of miR-34b in their blood plasma, indicating the potential of miRNAs as biomarkers (Gaughwin et al. 2011).

In the hippocampus of Alzheimer's disease (AD) patients as well as in the APPPS121 mouse model miR-34c was significantly increased. This miRNA targets Sirt1, a protein that is involved in memory function and learning (Zovoilis et al. 2011). Another miRNA deregulated in $A D$ is miR-29a which showed decreased expression in brains of patients compared to controls (Hébert et al. 2008; Shioya et al. 2010). miR-29a is regulating the protein expression of BACE-1, a protein that is involved in the cleavage of amyloid precursor protein (APP) and generation of toxic amyloid $\beta(A \beta)$-species. Thus, down regulation of miR-29a might lead to aberrant BACE-1 and therefore to increased A $\beta$ levels (Hébert et al. 2008). Decrease in miR-107 in AD at an early stage of pathology could be shown in different studies; interestingly this miRNA is also involved in the regulation of BACE-1 expression (Nelson \& Wang 2010; Wang et al. 2008). Additionally, several studies report that miRNA levels in CSF and plasma are changed in AD patients, suggesting a potential role as a diagnostic marker (Alexandrov et al. 2012; Cogswell et al. 2008; Müller et al. 2014).

Deregulation in miRNA expression is also shown in the brain of PD patients. miRNA profiling showed decreased expression of miR-34b and miR-34c in the amygdala, frontal cortex, SN and cerebellum of PD patients in motor stages (Braak stages 4 \& 5) as well as in pre-motor stages (Braak stages 1-3) of the disease. This reduction in miR-34b and miR-34c was coupled to an altered mitochondrial function, oxidative stress and reduction in cellular ATP content (Miñones-Moyano et al. 2011). miR-133b is important for dopaminergic neurons and usually enriched in the midbrain but markedly decreased in the midbrain of PD patients (Kim et al. 2007). It plays a crucial role in dopaminergic neuron development (see 1.3.3) and thus it is reasonable to suggest its involvement in the pathogenesis of PD. 
In addition to these profiling studies, different miRNAs have been discovered that regulate PD-associated genes. Expression of alpha-synuclein, a protein that plays a critical role in the formation of familial PD and is found in Lewy bodies, is post-transcriptionally regulated by miR-7. In the MPTP mouse model for PD as well as in a cell culture model using $\mathrm{MPP}^{+}$-toxicity miR-7 is down regulated, which could explain the increased alphasynuclein expression in these models. Furthermore, the authors could show that cells are protected by miR-7 from alpha-synuclein mediated oxidative stress (Junn et al. 2009). This interaction was confirmed in cortical neurons and HEK cells (Doxakis 2010). Another miRNA that regulates alpha-synuclein expression in cortical cell culture and HEK cells is miR-153 (Doxakis 2010). Increased alpha-synuclein expression could also be caused by increased fibroblast growth factor 20 (FGF20) expression resulting from a single nucleotide polymorphism (SNP) in the FGF20 gene that disrupts the binding side for miR433. These kind of SNP in the FGF20 gene is associated with a higher risk for PD (Wang et al. 2008). In Drosophila dopaminergic neurons it was shown that pathogenic LRRK2 inhibits let-7 and miR-184* which leads to increased expression of the transcription factors E3F1 and DP resulting in cell death. When let-7 and miR-184* were inhibited artificially in wildtype flies this resulted in a phenotype similar to animals carrying pathogenic LRRK2 (Gehrke et al. 2010). Beside the miRNAs that have a direct regulatory effect on PD-associated genes, there are also miRNAs associated with pathways affected in PD. Among others miR-98, miR-124, miR-130, miR-142 and miR-204 might be regulators of genes involved in the autophagy-lysosomal pathway (Jegga et al. 2011), regarding the importance of this pathway in PD it is possible that these miRNAs play a critical role in PD pathogenesis.

\subsubsection{The role of miRNAs in dopaminergic neuron biology}

In order to develop new therapeutic strategies for PD it is critical to understand the factors involved in midbrain dopaminergic neuron development, maintenance and also degeneration. It was demonstrated by several studies that miRNAs are important for the development and survival of neurons (De Pietri Tonelli et al. 2008; Makeyev et al. 2007; Olde Loohuis et al. 2012). The crucial role of miRNAs especially for dopaminergic 
neuron development and survival was demonstrated in vitro and in vivo with Dicer deficient models. siRNA-mediated Dicer knockdown in Drosophila led to loss of dopaminergic neurons and deficits in motor behavior (Gehrke et al. 2010). In murine ES cells elimination of miRNAs by Dicer depletion resulted in inhibited differentiation and loss of TH-positive (differentiated) cells under appropriate differentiation signals compared to a wildtype control ES cell line. When Dicer deficient ES cells were transfected with small RNAs isolated from mouse embryonic midbrain the phenotype was rescued (Kim et al. 2007). A conditional knockout of dicer in post-mitotic dopaminergic neurons led to a progressive loss of midbrain TH-positive neurons and their striatal projections that became first apparent in animals 2 weeks of age and was almost complete in 6 week old animals. This was accompanied by induction of apoptosis in the SN and dramatically reduced locomotion in the open field test (Kim et al. 2007). Wnt1cre-mediated conditional Dicer knockout in mice led to malformation of the midbrain and the cerebellum and an almost complete elimination of midbrain dopaminergic neurons due to impaired differentiation confirming the importance of miRNAs for dopaminergic neurons (Huang et al. 2010).

Midbrain enriched miR-133b is deficient in the midbrain of PD patients and other dopaminergic neuron deficient models (6-OHDA mice and Aphakia mice deficient for Ptx3 (Hwang et al. 2003)). Even though Aphakia mice maintain a population of dopaminergic neurons within the VTA they showed a prominent miR-133b deficiency, suggesting that miR-133b expression is directly regulated by the Pitx3 transcription factor. This was confirmed by an increase in miR-133b after Pitx3 overexpression in ES cells. Overexpression of miR-133b in ES cells and primary midbrain neuronal cultures (PMN) resulted in a significant decrease of the dopamine marker DAT, whereas early differentiation markers like Nurr1 appeared unaltered. Also dopamine release in miR$133 \mathrm{~b}$ overexpressing cultures was decreased. Inhibition of miR-133b by 2'OMe-modified RNA oligonucleotides in this cell culture models led to an increased expression of DAT and TH. The authors could show that Pitx3 is a target for miR-133b, thus miR-133b and Pitx 3 create a negative feedback loop in which Pitx3 increases miR-133b expression, whereas miR-133b inhibits Pitx3 translation. This results in the regulation of the final differentiation of midbrain dopaminergic neurons and their activity (Kim et al. 2007). Interestingly, these effects could not be observed in a miR-133b null mutant mouse 
model, which showed normal development and function of dopaminergic neurons in the midbrain, suggesting that maybe other members of the miR-133 family compensate for the loss of miR-133b or that other compensatory mechanisms are involved (Heyer et al. 2012).

\subsubsection{The potential of miRNAs providing neuronal protection and regeneration}

Despite that the role of miRNAs in central nervous system function is not yet fully understood there are already numerous studies indicating that they play a role in neuroprotection and regeneration. The neuroprotective effect of miRNAs is best studied in stroke and epilepsy models; nevertheless it was also shown in models of neurodegenerative diseases, e.g. HD. Increased levels of miR-22 decreased neurodegeneration in primary cortical and primary striatal neuron cultures treated with a mutated human huntingtin fragment. Additionally, in neuronal cultures exposed to a complex II/III inhibitor increased miR-22 levels led to a higher number of surviving neurons. The beneficial effects of increased miR-22 levels are mediated by a reduced caspase activation and targeting of the pro-apoptotic factors MAPK14/p38 and Tp53inp1. Interestingly, it was shown that miR-22 is downregulated in the brains of $A D$ and HD patients (Jovicic et al. 2013). Viral overexpression of miR-22 resulted in reduced inflammation and neuroprotection in a rat model of focal cerebral ischemia and reperfusion as well as in primary cortical neurons (Yu et al. 2015). Neuronal injury in stroke and neurodegenerative disorders is e.g. mediated by overstimulation of ionotropic glutamate receptors due to extracellular accumulation of glutamate. This response is regulated by miR-223, which targets glutamate receptor subunits. Increased miR-223 was neuroprotective in a mouse model for transient global ischemia, whereas inhibition of miR-223 led to increased neuronal apoptosis (Harraz et al. 2012). In a mouse model for epilepsy, seizure preconditioning by kainic acid led to upregulation of 25 miRNAs, among them miR-184. Inhibition of miR-184 expression in preconditioned animals resulted in a significant increase of seizure-induced neuronal cell death after status epilepticus. The authors conclude that the increase in miR-184 levels upon preconditioning contributes to the neuroprotective effects of seizure preconditioning and may contribute to epileptic 
tolerance development (McKiernan et al. 2012). Another example for miRNAs involved in epilepsy is miR-134. miR-134 is up regulated in animal models and patients with temporal lobe epilepsy, inhibiting miR-134 in the hippocampus of mice by antagomirs, rendered mice refractory to seizures and decreased neuronal injury after status epilepticus (Jimenez-Mateos et al. 2012). A study on the interplay between miR-125b and tauphopshorylation revealed miR-125b potential role in AD pathology. miR-125b expression is increased in the brains of $A D$ patients, leading to the assumption that inhibition of this miRNA in AD might have neuroprotective effects (Banzhaf-Strathmann et al. 2014).

Regarding the regeneration of nervous system structures there are indications that miRNAs are involved in regeneration of axons and synapses. In a mouse model of spinal cord injury miR-486 was shown to be up regulated. miR-486 directly targets NeuroD6 which is involved in the regulation of ROS homeostasis. Inhibition of miR-486 expression induced the expression of NeuroD6 and led to functional recovery in injured animals (Jee et al. 2012). Another example for miRNAs involved in neurite regeneration is miR-222. Increased miR-222 resulted in increased axonal outgrowth after sciatic nerve transection in rats by targeting PTEN (Zhou et al. 2012). Regarding regeneration in neurodegenerative disorders it was shown in a mouse model of ALS that miR-206 significantly delays disease progression. Furthermore, it is required for the regeneration of neuromuscular synapses after nerve injury (Williams et al. 2009).

Even though the role of miRNAs in neuroprotection and neuroregeneration is still emerging, there is already evidence that these small non-coding RNAs are powerful regulators of major pathways in the healthy and diseased nervous system. 


\subsection{Aims of this thesis}

Even though in recent years miRNAs in the context of neurodegenerative disorders were extensively studied, their role in Parkinson's disease is still not fully elucidated. Understanding miRNA-dependent regulations in dopaminergic neuron biology and PD can provide valuable insights into PD pathogenesis and also might lead to new therapeutic strategies for neuroprotection and regeneration of the nigrostriatal system. Thus, the aim of my work was to elucidate miRNA expression changes in primary midbrain neurons (PMN) upon development and neurite outgrowth and in the degenerating and regenerating substantia nigra. Furthermore, I wanted to exploit the neuroprotective and neuroregenerative potential of miRNAs in dopaminergic neurons in vitro and in vivo. Therefore, I formulated the following questions:

1. How does miRNA expression change in PMNs during development and neurite outgrowth? Does the pro-dopaminergic growth factor GDNF have influence on miRNA expression in PMNs?

In order to address these questions small RNA sequencing and differential expression analyses of (GDNF-treated) PMN cultures at different developmental stages was performed. As we assume that the cellular mechanisms that drive neurite outgrowth and differentiation during development are the same as those that play a role in regenerative responses, this study was performed with the goal to find miRNAs with a therapeutic potential.

2. How does miRNA expression in the midbrain change during degeneration and regeneration of the nigrostriatal system in the 6-OHDA mouse model for PD?

For analyses of miRNA expression changes during degeneration and regeneration of the nigrostriatal system we employed the 6-OHDA mouse model for PD and performed small RNA sequencing of the midbrain's small non-coding RNAs and differential expression analyses to get a better understanding of the mechanisms involved in degeneration and regeneration of the nigrostriatal system in vivo. 
3. Could changes in the levels of specific miRNA lead to protection and regeneration of dopaminergic neurons in vitro and in vivo?

In order to investigate the influence of increased levels of specific miRNAs, I used synthetic miRNA mimics and investigated their effects on dopaminergic neurons in PMNs in vitro and the MPTP mouse model for PD in vivo. 


\section{Materials and Methods}

\subsection{Materials}

\subsubsection{Reagents}

6-OHDA

$0.9 \%$ Saline

APS

Acrylamide

B-27 Supplement

Bepanthene

Bromphenol blue

BSA

Citric acid $\left(\mathrm{C}_{6} \mathrm{H}_{8} \mathrm{O}_{7} * \mathrm{H}_{2} \mathrm{O}\right)$

DAKO Diluent

DAPI

DEPC

DEPC water

Dermabond

DPX

EDTA

Ethanol absolute

FCS

Glucose

Glutamine

Glycerol

Glycine

GlycoBlue Coprecipitant

$\mathrm{H}_{3} \mathrm{BO}_{3}$

HBSS

HEPES
Sigma Aldrich (Taufkirchen, Germany)

Braun (Melsungen, Germany)

Sigma Aldrich (Taufkirchen, Germany)

Applichem (Darmstadt, Germany)

Gibco (Karlsruhe, Germany)

Braun (Melsungen, Germany)

Sigma Aldrich (Taufkirchen, Germany)

Applichem (Darmstadt, Germany)

Roth (Karlsruhe, Germany)

DAKO (Hamburg, Germany)

Sigma Aldrich (Taufkirchen, Germany)

Sigma Aldrich (Taufkirchen, Germany)

Sigma Aldrich (Taufkirchen, Germany)

Ethicon (Norderstedt, Germany)

Fluka (Steinheim, Germany)

Applichem (Darmstadt, Germany)

Applichem (Darmstadt, Germany)

Biochrom; PAA (Berlin; Pasching, Germany)

Merck (Darmstadt, Germany)

Lonza (Cologne, Germany)

Roth (Karlsruhe, Germany)

Applichem (Darmstadt, Germany)

ThermoFisher Scientific (Waltham, MA, USA)

Sigma Aldrich (Taufkirchen, Germany)

Gibco (Karlsruhe, Germany)

Applichem (Darmstadt, Germany) 


\begin{tabular}{|c|c|}
\hline HPLC water & Merck (Darmstadt, Germany) \\
\hline Ketamine & Medistar (Ascheberg, Germany) \\
\hline Laminin & Sigma Aldrich (Taufkirchen, Germany) \\
\hline L-AA & Sigma Aldrich (Taufkirchen, Germany) \\
\hline Luminol & Calbiochem (Darmstadt, Germany) \\
\hline Methanol & Applichem (Darmstadt, Germany) \\
\hline Mineral oil & Sigma Aldrich (Taufkirchen, Germany) \\
\hline Mowiol - 488 & Sigma Aldrich (Taufkirchen, Germany) \\
\hline MPTP hydrochloride & Sigma Aldrich (Taufkirchen, Germany) \\
\hline $\mathrm{MPP}^{+}$ & Sigma Aldrich (Taufkirchen, Germany) \\
\hline $\mathrm{Na}_{2} \mathrm{~B}_{4} \mathrm{O}_{7} * 10 \mathrm{H}_{2} \mathrm{O}$ & Sigma Aldrich (Taufkirchen, Germany) \\
\hline $\mathrm{NaO}_{2} \mathrm{C}_{2} \mathrm{H}_{3}$ & Roth (Karlsruhe, Germany) \\
\hline $\mathrm{NaHCO}_{3}$ & Merck (Darmstadt, Germany) \\
\hline $\mathrm{NaN}_{3}$ & Sigma Aldrich (Taufkirchen, Germany) \\
\hline $\mathrm{NaO}_{2} \mathrm{C}_{2} \mathrm{H}$ & Roth (Karlsruhe, Germany) \\
\hline $\mathrm{NaOH}$ & Applichem (Darmstadt, Germany) \\
\hline Neurobasal - A medium & Gibco (Karlsruhe, Germany) \\
\hline NGS & Biochrom; PAA (Berlin; Pasching, Germany) \\
\hline $\mathrm{NH}_{4} \mathrm{Cl}$ & Merck (Darmstadt, Germany) \\
\hline Non - fat dried milk & Applichem (Darmstadt, Germany) \\
\hline$p$ - cumaric acid & Applichem (Darmstadt, Germany) \\
\hline PFA & Applichem (Darmstadt, Germany) \\
\hline PBS & Applichem (Darmstadt, Germany) \\
\hline PLO & Sigma Aldrich (Taufkirchen, Germany) \\
\hline Precision Plus Dual Color Standard & BIO-RAD (Munich, Germany) \\
\hline SDS & Applichem (Darmstadt, Germany) \\
\hline Sodium octosulfonic acid & Fluka (Steinheim, Germany) \\
\hline Sterofundin & Braun (Melsungen, Germany) \\
\hline Sucrose & Applichem (Darmstadt, Germany) \\
\hline TEMED & Roth (Karlsruhe, Germany) \\
\hline Thionine acetate & Sigma Aldrich (Taufkirchen, Germany) \\
\hline TRI Reagent & Sigma Aldrich (Taufkirchen, Germany) \\
\hline
\end{tabular}


Tris base

Tris $\mathrm{HCl}$

TritonX 100

Trypsin

Tween20

Xylazine

Xylene

\subsubsection{Kits}

DAB Peroxidase Substrate Kit SK-4100

miScript II RT Kit

miScript SYBR Green PCR Kit

TruSeq Small RNA Sample Prep Kit
Applichem (Darmstadt, Germany)

Applichem (Darmstadt, Germany)

Applichem (Darmstadt, Germany)

Biochrom; PAA (Berlin; Pasching, Germany)

Applichem (Darmstadt, Germany)

Ecuphar (Greifswald, Germany)

Sigma Aldrich (Taufkirchen, Germany)

VECTASTAIN ABC Peroxidase Standard Kit PK-4000 Biozol (Eching, Germany)

\subsubsection{Buffers, solutions and cell culture medium}

Antibody solution for immunohistochemistry (IHC): $1 \%$ NGS, $0.1 \%$ TritonX 100 in PBS

Primary antibody solution for Western blot: $5 \%$ BSA in TBS-T

APS (10 \%) solution: Ammonium persulfate in distilled $\mathrm{H}_{2} \mathrm{O}$

Blocking solution for IHC: 10 \% NGS, $0.1 \%$ TritonX 100 in PBS

Blocking solution for Western blot: $5 \%$ non-fat dried milk in TBS-T

Borate buffer 100mM: solution 1: $19.7 \mathrm{~g} \mathrm{Na}_{2} \mathrm{~B}_{4} \mathrm{O}_{7} * 10 \mathrm{H}_{2} \mathrm{O}$ in $500 \mathrm{ml}$ distilled water; solution 2: $3.09 \mathrm{~g} \mathrm{H}_{3} \mathrm{BO}_{3}$ in $500 \mathrm{ml}$ distilled water. Adjust the $\mathrm{pH}$ of solution 2 with solution 1 to 8.5

Culture medium: 2 \% B27-supplement, 1 \% glutamine, $0.1 \% 200$ mM L-AA in NeurobasalA medium

DAPI solution: $1 \mu \mathrm{g} / \mathrm{ml}$ DAPI in PBS 
ECL-1: $10 \mu \mathrm{l} / \mathrm{ml} 250 \mathrm{mM}$ luminol, $4.4 \mu \mathrm{l} / \mathrm{ml} 90 \mathrm{mM}$-cumaric acid, $100 \mu \mathrm{l} / \mathrm{ml} 1 \mathrm{M}$ Tris pH 8.5 in distilled water

ECL-2: $0.9 \mu \mathrm{l} / \mathrm{ml} 30 \% \mathrm{H}_{2} \mathrm{O}_{2}, 100 \mu \mathrm{l} 1 \mathrm{M}$ Tris $\mathrm{pH} 8.5$ in distilled water

Gelelectrophoresis buffer: 192 mM glycine, 0.1 \% SDS, 25 mM Tris HCl, pH 8.3

HPLC buffer: $6.973 \mathrm{~g} / \mathrm{I} \mathrm{NaO} \mathrm{C}_{2} \mathrm{H}_{3}, 7.365 \mathrm{~g} / \mathrm{I} \mathrm{C}_{6} \mathrm{H}_{8} \mathrm{O}_{7} * \mathrm{H}_{2} \mathrm{O}, 0.105 \mathrm{~g} /$ I sodium octosulfonic acid, $0.048 \mathrm{~g}$ EDTA. Adjust $\mathrm{pH}$ to 4.3 using $1 \mathrm{M} \mathrm{C}_{6} \mathrm{H}_{8} \mathrm{O}_{7}, 105 \mathrm{ml} / \mathrm{I} \mathrm{CH} 4 \mathrm{O}$ in $800 \mathrm{ml} \mathrm{HPLC}$ water

Laemmli buffer: 312.5 mM Tris pH 6.8, 10 \% SDS, 50 \% Glycerin, 0.005 \% Bromphenolblau, $100 \mathrm{mM}$ DTT

Mowiol: 6 g glycerol, $2.4 \mathrm{~g}$ mowiol, $6 \mathrm{ml} \mathrm{H} \mathrm{H}_{2} \mathrm{O}, 12 \mathrm{ml}$ Tris $\mathrm{pH} 7.2$

PBS: $9.5 \mathrm{mg} / \mathrm{ml}$ PBS in distilled water

PBS-T: $0.1 \%$ TritonX 100 in PBS

PFA (4 \%) solution: $40 \mathrm{mg} / \mathrm{ml}$ PFA, $9.5 \mathrm{mg} / \mathrm{ml}$ PBS, 1-3 pellets $\mathrm{NaOH}$ in distilled water

RIPA buffer: $10 \mathrm{mM}$ Hepes, $142 \mathrm{nM} \mathrm{KCl}, 5 \mathrm{mM} \mathrm{MgCl}, 2.1 \mathrm{mM}$ EGTA and IGEPAL including complete proteasome inhibitor and phosphatase inhibitor

Running phase gel (10 \%): $2.83 \mathrm{ml} 30 \%$ acrylamide bisacryl, 2.1254 x Tris pH 8.8, $3.54 \mathrm{ml}$ distilled water, $4.25 \mu$ I TEMED, $42.5 \mu \mathrm{l} 10 \%$ APS

Secondary antibody solution: $5 \%$ non-fat dried milk in TBS-T

Stacking phase gel: $0.65 \mathrm{ml} 30 \%$ acrylamid bisacryl, $1.25 \mathrm{ml} 4 \times$ Tris $\mathrm{pH}$ 6.8, $3.05 \mathrm{ml}$ distilled water, $5 \mu$ I TEMED, $25 \mu \mathrm{l} 10 \%$ APS

TBS: $10 \mathrm{mM}$ Tris $\mathrm{HCl}, 150 \mathrm{mM} \mathrm{NaCl}$ in distilled water

TBS-T: $0.1 \%$ Tween20 in TBS, pH 7.6

Transfer buffer: $192 \mathrm{mM}$ glycine, $20 \%$ methanol, $25 \mathrm{mM}$ Tris $\mathrm{HCl}, \mathrm{pH} 8.3$

Tris: $10 \mathrm{mM}$ Tris-buffered saline $\mathrm{pH} 8.0$

\subsubsection{Antibodies}

\section{Primary antibodies}

Anti-Akt, from rabbit, polyclonal (Cell Signaling Technology, Cambridge, UK)

Anti-phospho-Akt, from rabbit, polyclonal (Cell Signaling Technology, Cambridge, UK) 
Anti-B-cell lymphoma 2 (anti-Bc/2), from mouse, monoclonal (Santa Cruz Biotech, Dallas, TX, USA)

Anti-dopamine transporter, from rat, polyclonal (Merck Millipore, Darmstadt, Germany) Anti-Glycerinaldehyd-3-phosphate dehydrogenase (anti-Gapdh), from mouse, monoclonal (HyTest Ltd., Turku, Finland)

Anti-FoxO1, from rabbit, monoclonal (Cell Signaling Technology, Cambridge, UK) Anti-FoxO3a, from rabbit, monoclonal (Cell Signaling Technology, Cambridge, UK) Anti-lonized calcium binding adapter molecule 1 (anti-Iba1), from rabbit, polyclonal (Wako Pure Chemical Industries, Neuss, Germany)

Anti-p44/42 Mitogen-activated protein kinase (anti-Erk1/2), from rabbit, polyclonal (Cell Signaling Technology, Cambridge, UK)

Anti-phospho-p44/42 Mitogen-activated protein kinase (anti-pErk1/2), from mouse, monoclonal (Cell Signaling Technology, Cambridge, UK)

Anti-phospho-S6 ribosomal protein (anti-phospho S6), from rabbit, polyclonal (Cell Signaling Technology, Cambridge, UK)

Anti-Signal transducer and activator of transcription 3 (anti-Stat3), from rabbit, polyclonal (Santa Cruz Biotech, Dallas, TX, USA)

Anti-TAO protein kinase 1 (anti-Taok1), from rabbit, polyclonal (Abcam, Cambridge, UK) Anti-ßTubulin (anti-Tubulin), from mouse, monoclonal (Sigma Aldrich, Taufkirchen, Germany)

Anti-tyrosine hydroxylase (anti-TH), from rabbit, polyclonal (Zytomed, Berlin, Germany)

\section{Secondary antibodies}

Anti-rabbit Alexa Fluor 488, from goat (Dianova, Hamburg, Germany)

Anti-rabbit biotinylated, from goat (Dianova, Hamburg, Germany)

Anti-rat biotinylated, from goat (Dianova, Hamburg, Germany)

Anti-rabbit Cy3, from goat (Dianova, Hamburg, Germany)

Anti-rabbit Dy Light 488, from goat (Dianova, Hamburg, Germany)

Anti-mouse horse radish peroxidase (HRP) conjugated, from goat (Cell Signaling Technology, Cambridge, UK)

Anti-rabbit HRP conjugated, from goat (Cell Signaling Technology, Cambridge, UK) 


\subsubsection{Equipment}

96 well micro test plate

6-, 12- and 24-well cell culture plates

Bead mill homogenizer Precellys 24

Bioanalyzer 2100

Brain matrix mouse

Cage incubator

Camera Legria HFM36

Ceramin beads $1.4 \mathrm{~mm}$

CFX96 Touch Real-Time PCR Detection System

Coulochem II electrochemical detector

Cover slides $24 \times 60 \mathrm{~mm}$

Cover slides diameter $12 \mathrm{~mm}$

Cryostat CM $3050 \mathrm{~S}$

Cryomatrix

Curix 60 Developer

Cyclone 25 PCR thermocycler

Electrophoresis power supply

Gilson pipettes

Glass capillaries

Guard cell

IVC animal cages

Micro-centrifuge 5415R

Micro Injector Nanoliter 2000 Pump Head Mini drill with $0.8 \mathrm{~mm}$ drill head

Mini-PROTEAN Tetra-Cell system

Mouse jaw holder with ear bars

Multipipette puller

NanoDrop 1000

Peristaltic perfusion pump

PVDC transfer membrane
Sarstedt (Nümbrecht, Germany)

Sarstedt (Nümbrecht, Germany)

Peqlab (Erlangen, Germany)

Agilent (Santa Clara, CA, USA)

World Precision Instruments (Berlin, Germany)

Okolab (Pozzuoli, Italy)

Canon (Krefeld, Germany)

Peqlab (Erlangen, Germany)

BIO-RAD (Munich, Germany)

ESA (Bedfort, CA, USA)

Menzel (Braunschweig, Germany)

Menzel (Braunschweig, Germany)

Leica Microsystems (Mannheim, Germany)

Thermo Scientific (Waltham, CA, USA)

Agfa (Cologne, Germany)

Peqlab (Erlangen, Germany)

GE Healthcare (Chalfton St. Gilles, UK)

Gilson (Villiers de belle, France)

World Precision Instruments (Berlin, Germany)

ESA (Bedfort, CA, USA)

Tecniplast (Hohenpeißenberg, Germany)

Eppendorf (Hamburg, Germany)

World Precision Instruments (Berlin, Germany)

Dremel (Leinfelden-Echterdingen, Germany)

BIO-RAD (Munich, Germany)

World Precision Instruments (Berlin, Germany)

World Precision Instruments (Berlin, Germany)

Thermo Scientific (Waltham, CA, USA)

Idex (Wertheim, Germany)

Applichem (Darmstadt, Germany) 
Spacer plates / short plates

Spectra / Por7 MWCO 1000 membranes

Stereotactic frame

SuperFrost Plus Microscope Slides

SYS Micro4 controller

Whatman blotting paper
BIO-RAD (Munich, Germany)

Carl Roth (Karlsruhe, Germany)

David Kopf Instruments (Tujunga, CA, USA)

Menzel (Braunschweig, Germany)

World Precision Instruments (Berlin, Germany)

GE Healthcare (Chalfton St. Gilles, UK)

\subsubsection{Software}

AxioVision 4.6 (Carl Zeiss Microimaging)

CFX Manager Software (BIO-RAD)

CorelDRAW X6 (Corel Corporation)

Cromeleon Chromatography Data System (Dionex by Thermo Fisher Scientific GmbH) ImageJ $1.47 \mathrm{v}$ (N.I.H.)

KyPlot 2.0 (KyensLab Incorporated)

Observer XT (Ethovision)

R 3.1.3 (R-project)

Sigma Plot 10.0 (Systat Software GmbH)

Stereo Investigator 9.0 (Micro Bright Field Inc.)

\subsection{Methods}

\subsubsection{Animal surgery, injections and tissue processing}

\subsubsection{Animals}

For preparation of primary midbrain neuron cultures female $\mathrm{C} 57 \mathrm{~B} / 6 / \mathrm{J}$ mice (Charles River or central animal facility, University Medicine Göttingen) were used as donor animals for embryonic day (E) 12.5 embryos.

All animals used for in vivo experiments were 8-10 weeks old male C57BI6/J mice (Charles River) that were housed in groups of 5 animals in individually ventilated cages 
(IVC; 365 × $207 \times 140 \mathrm{~mm}$ ). Animals were housed under a 12 hours light dark/ cycle with food and water ad libitum. Three days prior to surgeries mice received metamizol (1.5 $\mathrm{mg} / \mathrm{ml}$ ) in drinking water to ensure optimal analgesic conditions. All experiments were performed in accordance to the national German animal protection law under the grant no. $13 / 1118$ approved by the local authorities.

\subsubsection{Stereotactic injection of 6-OHDA}

To prepare the micro injector a self-pulled glass capillary was filled with mineral oil and connected to the micro injector. Withdrawal of substances was performed under control of a SYS-Micro4 Controller. An air bubble of $500 \mathrm{nl}$ was withdrawn in order to separate the mineral oil from the substance that should be injected. 6-Hydroxydopamine solution (6-OHDA) was freshly prepared by dissolving the substance in $0.2 \%$ L-ascorbic acid (LAA) in phosphate-buffered saline (PBS) at a concentration of $2 \mu \mathrm{g} / \mu \mathrm{l}$ and withdrawn into the capillary.

Animals were anesthetized with a mixture of ketamine $(150 \mathrm{mg} / \mathrm{kg}$ body weight) and xylazine (10 mg/kg body weight) in Sterofundin infusion solution. To control anesthesia toe pinch and eyelid-closure reflexes were checked. The mouse was placed in a stereotactic frame and fixed by ear bars and jaw holder, the eyes were protected with Bepanthen eye salve (figure 2.2/1 a). The scalp was desinfected and a longitudinal cut along the midline was executed with a scalpel. The skin and connective tissue were carefully held at the sites by small hooks, so that bregma and lambda were exposed (figure 2.2/1 a). The coordinates for injection in the right striatum relative to bregma were: Anterior - posterior $(\mathrm{AP})+0.04 \mathrm{~cm}$; medio - lateral $(\mathrm{ML})-0.18 \mathrm{~cm}$; dorso - ventral (DV) $-0.35 \mathrm{~cm}$ (Alvarez-Fischer et al. 2008; Paxinos \& Franklin 2004). The bregma and lambda coordinates were measured and drilling coordinates were set. A minimal trepanation of the skull was performed using an electrical mini drill equipped with a 0.8 $\mathrm{mm}$ drill head. The capillary was brought to the right position and depth and $2 \mu \mathrm{l}$ 6-OHDA solution were injected at a flow rate of $0.5 \mu \mathrm{l}$ per minute. After the injection the capillary was left in place for 4 minutes in order to prevent reflux via the injection tract. After the needle was removed the scalp was closed using surgical tissue adhesive (Dermabond). 
The animal received a subcutaneous injection of $1 \mathrm{ml} 37^{\circ} \mathrm{C}$ warm Sterofundin and was placed on a warming pad until awakening. In the subsequent days the animal was treated with metamizol, monitored closely and received mashed food.
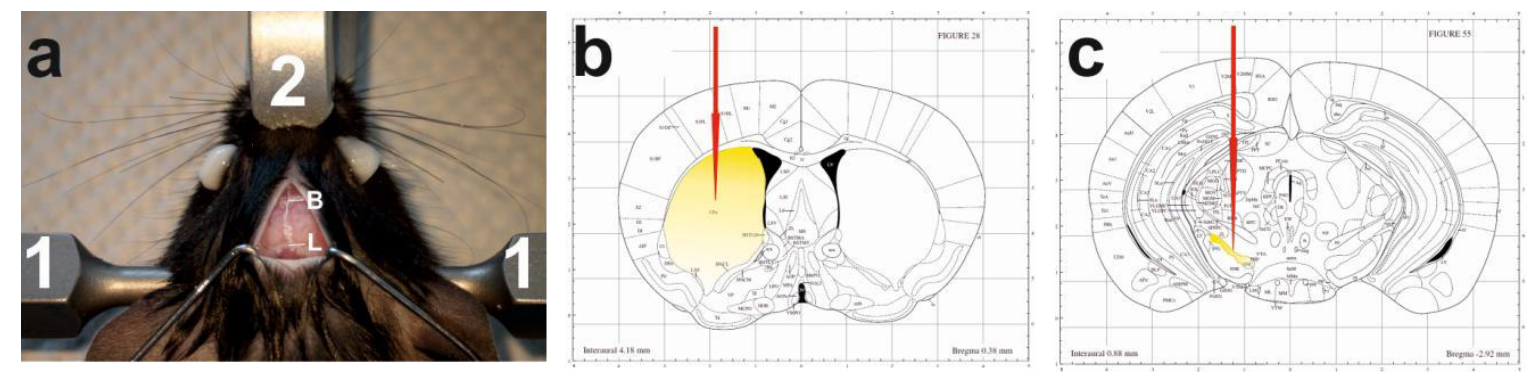

Figure 2.2/1: Illustration of the injection sites for stereotactic injection. a shows the animal fixed with ear bars (1) and jaw holder (2), the skin is opened and Bregma (B) and Lambda (L) are exposed. b and $\mathbf{c}$ illustrate the injection coordinates in the putaminal striatum (yellow area in b) for the 6-OHDA injection and the midbrain with the SNpc (yellow area in c) for the oligo injection (adapted from Paxinos \& Franklin, 2001).

\subsubsection{Stereotactic injection of synthetic miRNA mimics or scrambled negative control siRNA}

For stereotactic injections of synthetic miRNA mimics for mmu-miR-182-5p and mmu-miR-183-5p or negative control scrambled siRNA (NC siRNA; facultative with fluorescent label) (Qiagen) in the right substantia nigra (SN) of mice, HPLC-purified miRNA mimics or siRNA ready for in vivo applications were employed. To prepare oligos for injection all handling was done under aseptic conditions. Sterile cold PBS was added to the oligo tube to a final concentration of $125 \mu \mathrm{M}(250 \mu \mathrm{M}$ or $50 \mu \mathrm{M}$ respectively for fluorescently labelled NC siRNA), mixed and incubated at RT for $3 \mathrm{~min}$. The solution was portioned in suitable aliquots; snap frozen and stored at $-80^{\circ} \mathrm{C}$ for not longer than a month. After thawing, oligo solutions were not re-frozen. Immediately before injections the oligos were thawed on ice. In order to form transfection complexes $1.35 \mu \mathrm{l} \mathrm{HiPerfect}$ transfection reagent per $10 \mu \mathrm{l}$ oligo were added, mixed intensively, left at RT for $5 \mathrm{~min}$ and placed on ice. Directly before filling of the capillary, the oligo - HiPerfect mixture was warmed in the hand for 1 min and mixed. 
The animal was anesthetized and prepared for surgery as described above. After fixation of the head and opening of the scalp the coordinates for an injection in the right SN relative to bregma were set (AP: $-0.29 \mathrm{~cm}$; ML: $-0.12 \mathrm{~cm}$; DV: $-0.45 \mathrm{~cm}$ (AlvarezFischer et al. 2008; Paxinos \& Franklin 2004)). $2 \mu$ oligo - HiPerfect mixture were injected at an injection rate of $150 \mathrm{nl}$ per minute. The needle was left in place for 4 min after the injection to prevent reflux via the trajectory tract. After removing the capillary, the animals were treated as mentioned above.

\subsubsection{A subchronic mouse model of PD - Intraperitoneal injections of 1-methyl- 4-phenyl-1,2,3,6-tetrahydropyridin (MPTP)}

In order to investigate the neuroprotective and -regenerative effect of increased mmu-miR-182-5p and mmu-miR-183-5p levels, the subchronic mouse model of PD was applied. Therefore the animals received intraperitoneal injections of $30 \mathrm{mg} / \mathrm{kg}$ body weight MPTP on 5 consecutive days beginning $48 \mathrm{~h}$ after injection of the respective oligo into the SN. For injections MPTP was dissolved in $0.9 \%$ saline, control animals received just saline. Handling of MPTP and MPTP injected mice was performed according to appropriate guidelines (Przedborski et al. 2001).

\subsubsection{Behavioral tests}

In order to assess motor behavior of animals after miRNA injections and MPTP treatment, mice had to perform the open field test and the cylinder rearing test 13 days after the first MPTP injection. All animal experiments were performed in a brightly lit room.

\subsection{Open field test}

General motor behavior was analyzed using the open field test. Animals were placed in the center of an $80 \mathrm{~cm} \times 80 \mathrm{~cm}$ open arena with side walls of $20 \mathrm{~cm}$ height for 5 
min. A camera was placed above the arena and recorded all behavior of the animal. Videos were analyzed using The Observer XT software and total distance moved, the time spent moving, and the relative time spent in the center of the arena were quantified. By this, the animals' movement was automatically tracked and the arena was fielded in to a $68 \mathrm{~cm} \times 68 \mathrm{~cm}$ sized center area surrounded by a $12 \mathrm{~cm}$ broad margin area.

\subsection{Cylinder rearing test}

In order to analyze a potential effect of miRNA mimic injection, forelimb use during natural exploratory behavior was assessed using the cylinder rearing test (Schallert et al. 2000; Tönges et al. 2012). To this, each animal was placed in a transparent $25 \mathrm{~cm}$ high Plexiglas cylinder that measured $11.5 \mathrm{~cm}$ in diameter. Behind the cylinder a $90^{\circ}$ angulated mirror was placed to allow observation of forelimb movements when the animal turns away from the observer. All behavior was recorded by a camera. The test duration was 5 minutes, beginning with the placement of the animal. During a full rear the first contact of a forelimb was recorded as an independent wall placement event for the respective limb. If the animal used a single paw to assist a rear the rating criteria were "right" and "left" according to the forelimb used. If the animal assisted a rear with both paws, the event was rated as "both" and if the rear was performed without forelimb use it was rated as "free". Rating of the recorded behavior was done manually.

\subsubsection{Animal euthanasia and tissue processing}

\subsection{Transcardial perfusion of mice}

For histological analysis of tissues, a rapid and thorough fixation of specimens is essential. For preservation of morphological structures and inhibition of biochemical processes that lead to degradation of cells transcardial perfusion with $4 \%$ paraformaldehyde (PFA) in PBS is the common method. To this, animals were deeply anesthetized by $\mathrm{CO}_{2}$ administration. After anesthesia the abdominal skin was opened by a longitudinal cut and removed. Then the thoracic diaphragm and the ribs were bilaterally 
cut to open the thorax. The heart was exposed, the pericardium removed and a perfusion cannula connected to a perfusion pump filled with ice-cold sterile PBS was inserted into the left ventricle of the still beating heart. Shortly after inserting the cannula the right auricle was opened by a small cut in order to allow the blood to void and enable the PBS to clean the whole cardiovascular system from blood, including the brain. Perfusion was performed with PBS for 5 min, followed by a 5 min perfusion with ice-cold $4 \%$ PFA in PBS to fixate the tissue. Optimal perfusion was indicated by a twitching of the tail and a color change of the liver from red to yellow-white. After perfusion, the brain was removed and transferred into $4 \%$ PFA in PBS for two days at $4^{\circ} \mathrm{C}$ for post-fixation. Afterwards, brains were transferred into $30 \%$ sucrose-PBS solution for another two days to dehydrate the tissue. Dehydrated brains were stored at $-80^{\circ} \mathrm{C}$ until cryosectioning.

\subsection{Cryosectioning of mouse brains}

PFA-fixed dehydrated still frozen brains were embedded in Cryomatrix coronally cryosectioned into $30 \mu \mathrm{m}$ thick sections using a Leica Cryostat CM3050S. Precisely the area of the striatum (Bregma AP +1.18 to $-0.70 \mathrm{~mm}$ ) and the midbrain (Bregma AP 2.18 to $-4.04 \mathrm{~mm}$ ) were cut and sections were stored free floating in $0.1 \%$ sodium azide in PBS at $4^{\circ} \mathrm{C}$ until immunohistochemistry.

\subsection{Preparation of the striatum and the substantia nigra from fresh brain tissue}

For neurochemical analysis of dopamine and its metabolites in the striatum or isolation of RNA or proteins from the SN the brain tissue should be native and not fixed. Here, animals were sacrificed by cervical dislocation. After decapitation, the brain was removed and the areas of interest were isolated, snap frozen in liquid nitrogen and stored at $-80^{\circ} \mathrm{C}$ till further processing. 


\subsubsection{Cell culture}

To investigate miRNA expression during development of primary neurons, the effect of glial cell-line derived neurotrophic factor (GDNF) on miRNA expression levels, as well as the effect of manipulation of miRNA expression on neurite growth, neuroprotection, neurite regeneration and protein expression, murine primary midbrain neuron cultures were employed. Donor animals were provided by the central animal facility of the UMG or Charles River.

\subsubsection{Preparation of cover slips and coating of culture plates}

For immunocytochemistry, primary midbrain neurons were seeded onto glass coverslips (diameter: $12 \mathrm{~mm}$ ). Those were cleaned and degreased by shaking in acetone for $5 \mathrm{~min}$, followed by 3 washing steps in pure ethanol for $30 \mathrm{~min}$ each. Subsequently the coverslips are rinsed 3 times in double distilled water. After drying the degreased coverslips were baked at $180^{\circ} \mathrm{C}$ for $4 \mathrm{~h}$.

Cell culture plates and coverslips were coated with poly-I-ornithine (PLO) and laminin under sterile conditions. To this, poly-l-ornithine in $0.15 \mathrm{M}$ sodium borate buffer $(1 \mathrm{mg} / \mathrm{ml})$ was diluted to a final concentration of $0.1 \mathrm{mg} / \mathrm{ml}$ in sterile double-distilled water and an adequate amount, depending on the size of the culture plate, pipetted into the wells of the culture plate, and incubated for at least $4 \mathrm{~h}$ at RT. Afterwards, the cell culture plates were rinsed 3 times with sterile water. Laminin $(1 \mu \mathrm{g} / \mathrm{ml})$ in Neurobasal culture medium without supplements was transferred to the wells and incubated for at least $1 \mathrm{~h}$ in an incubator at $37^{\circ} \mathrm{C}$ with $5 \% \mathrm{CO}_{2}$ and $95 \%$ humidity. Subsequently, laminin solution was removed and wells were washed 3 times with Neurobasal culture medium without supplements.

\subsubsection{Primary midbrain neuron culture}

Primary midbrain neuron cultures were prepared under serum free conditions from embryonic day 12.5 (E12.5) C57BI6/J mice. Pregnant female mice were sacrificed with carbon dioxide $\left(\mathrm{CO}_{2}\right)$ and the uteri were transferred into cold Hank's balanced salt 
solution (HBSS). The following steps were all performed under aseptic conditions. The embryos were removed and placed into ice cold HBSS. The ventral mesencephalon was isolated and the meninges removed. Midbrains were collected in ice cold HBSS and centrifuged at $800 \mathrm{rpm}$ for $1 \mathrm{~min}$. The pellet was then incubated in $1 \mathrm{ml} 0.25 \%$ trypsin for $12 \mathrm{~min}$ at $37^{\circ} \mathrm{C}$. After addition of $50 \mu$ DNAse $(5 \mathrm{mg} / \mathrm{ml})$ the tissue was centrifuged for 2 $\mathrm{min}$ at $800 \mathrm{rpm}$. Afterwards the supernatant was removed and $1 \mathrm{ml}$ fetal calf serum (FCS) was added and the tissue was dissociated by gentle trituration with a fire polished Pasteur pipette. The cell suspension was centrifuged for $4 \mathrm{~min}$ at $800 \mathrm{rpm}$ and the resulting pellet was resuspended in culture medium. Neurons were counted with a Neubauer counting chamber and were seeded into poly-I-ornithine and laminine coated cell culture plates or glass coverslips.

\subsubsection{Transfection of primary midbrain neurons}

In order to achieve elevated miRNA levels, primary midbrain neurons were transfected with a synthetic miRNA mimic or a scrambled negative control siRNA on day in vitro (DIV) 1. We employed synthetic miRNA mimics, which mimic the mature endogenous miRNA after transfection for mmu-miR-182-5p and mmu-miR-183-5p (see sequences below). As a control we used a validated scrambled negative control siRNA (NC siRNA; AllStars Negative Control siRNA). This scrambled siRNA has no homology to any known mammalian gene, for its validation several cell based assays as well as Affymetrix Gene Chip arrays were employed and resulted in minimal nonspecific effects on gene expression and phenotype. To ensure that observed effects are not the result of increased miRNA levels per se we used a mimic for mmu-miR-1a-3p as an additional control. This miRNA has been shown to have no effect on neurite growth before (Vo et al. 2005). To compare the effect of elevated miRNA levels with the effect of GDNF treatment, an experimental group was included were cells were transfected with NC siRNA and treated with GDNF at a final concentration of $10 \mathrm{ng} / \mathrm{ml}$. In order to form transfection complexes, miRNA mimics or NC siRNA were diluted in culture medium and HiPerfect transfection reagent was added ( $2 \mu \mathrm{l}$ per $100 \mu \mathrm{l}$ transfection complexes). The amount of the different components was dependent on cell density and the size of the 
cell culture plate (table 2.2/1). The solution was mixed extensively for $1 \mathrm{~min}$ and incubated for $10 \mathrm{~min}$ at RT to allow the formation of transfection complexes. For optimization of transfection, cells were transfected with miRNA mimics or NC siRNA at a final concentration of $100 \mathrm{pM}, 5 \mathrm{nM}$ and $50 \mathrm{nM}$. All experiments concerning the effect of miRNA mimics were conducted at a final oligo concentration of $5 \mathrm{nM}$.

After incubation, the mixture was gently added droplet-wise to the medium of the cultured midbrain neurons. Three hours after addition of the transfection complexes, half of the culture medium was removed and the same amount fresh culture medium was added.

Table 2.2/1: Cell numbers in different plate formats

\begin{tabular}{l|l|l|l} 
Plate format & Cell number & Cell density per $\mathrm{cm}^{2}$ & Final amount of medium \\
\hline 6 well & $3 \times 10^{6}$ & $3 \times 10^{5}$ & $2400 \mu \mathrm{l}$ \\
12 well & $1.5 \times 10^{6}$ & $3 \times 10^{5}$ & $1200 \mu \mathrm{l}$ \\
24 well & 650.000 & $3 \times 10^{5}$ & $600 \mu \mathrm{l}$
\end{tabular}

\subsubsection{1-Methyl-4-phenylpyridinium (MPP ${ }^{+}$treatment}

To investigate the potential neuroprotective effect of increased mmu-miR-182 and mmu-miR-183 levels, transfected midbrain neuron cultures were treated with MPP . $^{+}$ $\mathrm{MPP}^{+}$is the active metabolite of the dopaminergic neurotoxin MPTP. In vivo, the lipophilic MPTP can cross the blood-brain-barrier and enter the brain tissues, where it is converted by the enzyme monoamine oxidase $\mathrm{B}(\mathrm{MAO}-\mathrm{B})$ in astrocytes to $\mathrm{MPP}^{+}$. $\mathrm{MPP}^{+}$has a strong selective toxicity for dopaminergic neurons, because its uptake into the cell is mediated by the dopamine transporter (DAT), which is present on this neuronal subtype. After entering the cell, $\mathrm{MPP}^{+}$accumulates in the mitochondria, where it inhibits $\mathrm{NADH}$ dehydrogenase (complex I) leading to a blockage of mitochondrial respiration, resulting in the formation of reactive oxygen species (ROS) and consequently to oxidative stress (Kopin 1992). 24 hours post-transfection (DIV2), $\mathrm{MPP}^{+}$was carefully added to the culture medium at a final concentration of $2 \mu \mathrm{M}$. For control groups the same amount of PBS was added to the culture medium. After $24 \mathrm{~h}$ (DIV3) the complete culture medium was 
removed and fresh culture medium without $\mathrm{MPP}^{+}$was added. Cells were cultured for another $24 \mathrm{~h}$ (DIV4), then rinsed with PBS and fixed with $4 \%$ PFA in PBS for 10 min at RT. Afterwards coverslips were rinsed with PBS and stored in PBS until staining.

\subsubsection{Scratch lesion}

For assessment of potential effects of elevated miRNA levels on neurite regeneration in dopaminergic neurons, a mechanical scratch lesion was applied to primary midbrain neuron cultures. One day post-transfection (DIV2) a $200 \mu$ l pipette tip was quickly scratched 2 times across each coverslip, resulting in a transection of neurites along the scratch border. Two days later (DIV4) coverslips were rinsed with PBS, fixed and stored until staining as mentioned above.

\subsubsection{Molecular biology and biochemical techniques}

\subsubsection{Isolation of total RNA from cell cultures and brain tissue}

For Isolation of total RNA from cell cultures and brain tissues TRI-reagent was used. TRI-reagent is a combination of phenol and guanidine isothiocyanate that allows simultaneous isolation of RNA, DNA and proteins from one sample (Simms et al. 1993). Unless otherwise noted all procedures were performed at RT. For isolation of total RNA from cell cultures $1 \mathrm{ml}$ TRI-reagent per $10 \mathrm{~cm}^{2}$ cell culture surface was employed. After removal of culture medium, cells were rinsed with cold PBS and $500 \mu \mathrm{l}$ TRI-reagent was given to each well and allowed to incubate for $3 \mathrm{~min}$. Afterwards the TRI-reagent-cellIysate was transferred to an Eppendorf cup, another $500 \mu \mathrm{l} \mathrm{TRI-reagent} \mathrm{were} \mathrm{added} \mathrm{and}$ the suspension was homogenized by pipetting repeatedly up and down. The suspension was allowed to stand for another $3 \mathrm{~min}$ to ensure a complete lysis of cell material. Subsequently, $100 \mu \mathrm{l}$ 1-Bromo-3-chlorpropane were added and the mixture was extensively mixed by shaking for $15 \mathrm{sec}$ and incubated for $3 \mathrm{~min}$ at RT. To allow separation of the organic-, aqueous- and interphase the mixture was centrifuged for 15 min at $12.000 \times$ g at $4^{\circ} \mathrm{C}$. The aqueous phase containing the RNA was transferred to a new 
tube whereas the organic- and interphase were stored at $-80^{\circ} \mathrm{C}$ for eventual protein isolation. $500 \mu \mathrm{l}$ isopropanol and 15-20 $\mu \mathrm{g}$ GlycoBlue were added to the aqueous phase, mixed vigorously and stored at $-20^{\circ} \mathrm{C}$ for at least 90 min or overnight (ON) to allow RNA precipitation. This was followed by centrifugation for $25-30 \mathrm{~min}$ at $12.000 \mathrm{x} \mathrm{g}$ at $4^{\circ} \mathrm{C}$. Afterwards the supernatant was carefully discarded and the RNA pellet was washed twice with $75 \%$ ethanol followed by 5 min centrifugation at $12.000 \times \mathrm{g}$ at $4^{\circ} \mathrm{C}$ and dissolved in 20-30 $\mu \mathrm{l} \mathrm{DEPC-} \mathrm{H}_{2} \mathrm{O}$.

For isolation of total RNA from brain tissue, frozen tissue was homogenized in 500 $\mu$ l TRI-reagent with the help of a sterile micropistil. After homogenization another $500 \mu \mathrm{l}$ TRI-reagent were added and the mixture was allowed to incubate for $5 \mathrm{~min}$. All following steps were similar to those for total RNA isolation from cell cultures.

\subsubsection{Preparation of protein lysates from cell cultures}

To prepare protein lysates for Western blotting culture medium was removed and cells were placed on ice and rinsed with cold $5 \%$ glucose solution. After removal of glucose solution ice-cold RIPA buffer was added to the cells and incubated for 10 min on ice to allow cell lysis. After incubation cells were scraped off the well using a silicon cell scraper and transferred into a tube. Lysates were sonicated on ice and protein concentration was measured using a BCA assay. After determination of protein concentrations Laemmli buffer containing $10 \%$ dithiothreitol (DTT) was added, followed by heating for $10 \mathrm{~min}$ at $95^{\circ} \mathrm{C}$.

\subsubsection{Isolation of proteins from brain tissue}

When brain tissue was used for simultaneous RNA and protein extraction, TRIreagent was used (see 2.2.3.1 for RNA protocol). For isolation of proteins from the organic phase after phase separation the remaining aqueous phase overlaying the interphase was carefully removed. The DNA from the interphase and organic phase was precipitated by addition of $300 \mu \mathrm{l} 100 \%$ ethanol. The sample was mixed by inversion and incubated for $5 \mathrm{~min}$ at RT. Subsequently it was centrifuged for $5 \mathrm{~min}$ at $2000 \times \mathrm{g}$ at $4^{\circ} \mathrm{C}$. 
The supernatant was transferred into a Spectrapor MW 1000 membrane, the DNA pellet was discarded. Samples were dialyzed against $0.1 \%$ SDS at $4^{\circ} \mathrm{C}$ overnight. After dialysis the white globular mass containing the protein was transferred into a new tube, resolved in $4 \mathrm{M}$ Urea in $0.5 \% \mathrm{SDS}$ and stored at $-20^{\circ} \mathrm{C}$ until addition of Laemmli buffer and Western blotting.

Protein lysates from brain tissue without prior RNA isolation were prepared when no RNA was needed. To this, the frozen tissue was homogenized manually in cold RIPA buffer using a micropistil, incubated for $10 \mathrm{~min}$ on ice, followed by sonication.

Protein concentrations were measured as described below, Laemmli buffer containing $10 \%$ DTT was added and samples were boiled at $95^{\circ} \mathrm{C}$ for $10 \mathrm{~min}$. Samples were stored at $-20^{\circ} \mathrm{C}$ until blotting.

\subsubsection{Determination of nucleic acid concentrations}

RNA concentration and purity were analyzed using a NanoDrop 1000 spectrophotometer by measuring the absorption at $260 \mathrm{~nm}$ (A260) according to manufacturer's instructions to determine RNA concentration and analyzing the 260/280 ratio and the $230 / 280$ ratio to check the RNA purity. Before RNA was used for preparation of small RNA libraries for sequencing, the RNA integrity was verified with Agilent RNA 6000 Nano Chip in the Agilent 2100 Bioanalyzer electrophoresis system. This system calculates the RNA integrity number (RIN) for a given sample. The RIN is an algorithm that assigns integrity values to RNA measurements. The RIN ranges from 1 to 10 , whereas 1 means the RNA is maximal degraded and 10 means that the RNA is intact. For preparation of small RNA libraries only samples with a RIN > 9 were used.

\subsubsection{Determination of Protein concentrations}

Protein concentrations were measured employing the Pierce BCA protein assay kit according to manufacturer's instructions. After 30 min incubation at $37^{\circ} \mathrm{C}$ absorption was measured at $562 \mathrm{~nm}$ (A562) using the Rainbow Spectra Tecan. 


\subsubsection{Reverse transcription}

Synthesis of complementary DNA (cDNA) from total RNA isolates was performed using the miScript II RT kit from Qiagen. This kit allows the reverse transcription of total RNA including miRNAs. Mature miRNAs are polyadenylated by poly $(A)$ polymerase and converted into cDNA by a reverse transcriptase with oligo-dt priming. For reverse transcription reactions $500 \mathrm{ng}$ total RNA were used. A reverse transcription master mix including 10 x HiSpec Buffer, 5 x Nucleics Mix and miScript Reverse Transcriptase Mix was prepared and added to $500 \mathrm{ng}$ total RNA in $6 \mu$ PCR-grade water. All steps were performed on ice. The total reaction volume was $10 \mu \mathrm{l}$ per reaction. The mixture was incubated in a Cyclone $25 \mathrm{PCR}$ thermocycler for $60 \mathrm{~min}$ at $37^{\circ} \mathrm{C}$ followed by a $5 \mathrm{~min}$ incubation step at $95^{\circ} \mathrm{C}$ to inactivate the miScript Reverse Transcriptase Mix. Resulting CDNA was diluted with $100 \mu \mathrm{l}$ PCR-grade water and stored at $-20^{\circ} \mathrm{C}$ until quantitative real time PCR.

\subsubsection{Quantitative real time PCR (qRT-PCR)}

In order to determine miRNA expression levels quantitative real time PCR was conducted in the CFX96 Touch Real-Time PCR Detection System. For miRNA expression analyses, optimized mature miRNA-specific primers (Qiagen, Hilden, Germany) and cDNA at a dilution of 1:10 were used (table 2.2/2). miRNA expression was normalized to the endogenous control RNU6. Quantitative real time PCR was carried out using the miScript SYBR Green PCR kit according to manufacturer's instructions with cycling conditions as described in table 2.2/3. A dissociation (melting) curve analysis was included in every run. Relative expression levels were calculated using the $\Delta \Delta \mathrm{Ct}$ method.

Table 2.2/2: miRNA specific primers used for qPCR

\begin{tabular}{l|l} 
Small RNA amplified & Primer \\
\hline mmu-miR-182-5p & Mm_miR-182_2 miScript Primer Assay \\
mmu-miR-183-5p & Mm_miR-183_1 miScript Primer Assay \\
RNU6 & Hs_RNU6-2_11 miScript Primer Assay
\end{tabular}


Table 2.2/3: qPCR cycling conditions

\begin{tabular}{l|l|l} 
Step & Time & Temperature \\
\hline Initial activation & $15 \mathrm{~min}$ & $95^{\circ} \mathrm{C}$ \\
3 Step cycling $(40 \mathrm{x}):$ & & \\
Denaturation & $15 \mathrm{~s}$ & $94^{\circ} \mathrm{C}$ \\
Annealing & $30 \mathrm{~s}$ & $55^{\circ} \mathrm{C}$ \\
Extension & $30 \mathrm{~s}$ & $70{ }^{\circ} \mathrm{C}$
\end{tabular}

\subsubsection{Small RNA sequencing and analysis of sequencing data}

Small RNA sequencing was performed in the lab of Prof. Dr. André Fischer (DZNE, Göttingen) on an Illumina HiSeq 2000 system. Small RNA libraries were prepared from 1 $\mu \mathrm{g}$ total RNA using the Illumina TruSeq Small RNA Sample Preparation kit. Primary analysis of sequencing data was performed by the groups of Prof. Dr. Stefan Bonn and Prof. Dr. André Fischer (DZNE, Göttingen).

For processing of sequencing data a customized software pipeline was used. For quality check and demultiplexing the CASAVA 1.8.2 software (Illumina) was used. For alignment the basic local alignment search tool (BLAST) was used. Reads were aligned to MiRBase version 19 with a cutoff score of $p<5 \times 10^{-7}$ and a single mismatch allowed.

In order to compare the miRNA expression levels between samples, a differential expression analysis was performed using $\mathrm{R}$ and the DESeq package. Heatmaps were created using the ggplot2 package.

Computational miRNA target prediction analysis was performed using TargetScanMouse 6.2 (Lewis et al. 2005). For functional annotation of predicted miRNA targets the data mining environment provided by the DAVID platform (Huang et al. 2009) was used. The functional annotation module was applied for gene ontology terms using an EASE score of 0.1 and a minimum number of 2 counts. For checking enrichment of miRNA targets in specific Kyoto Encyclopedia of Genes and Genomes (KEGG) pathways DIANA mirPath was employed (Papadopoulos et al. 2009). In order to search for already experimentally validated target genes, the miRTarBase database (Hsu et al. 2014) was used. 


\subsubsection{Sodium dodecyl sulfate-polyacrylamide gel electrophoresis (SDS-PAGE)}

In order to separate proteins according to their molecular weight SDS-PAGE was performed. During SDS-PAGE proteins are separated by gel electrophoresis in presence of the denaturating detergent SDS, which allows a separation by size that excludes the effects of electric charge and conformation. In order to collect proteins with different sizes to allow movement through the gel at the same rate, a $5 \%$ stacking phase polyacrylamide gel with a $\mathrm{pH}$ of 6.8 was polymerized over the resolving gel. Depending on the size of the protein of interest, resolving phase gels with a polyacrylamide content between 8 and $15 \%$ and a pH of 8.8 were used. For electrophoresis the BIO-RAD MiniPROTEAN Tetra-Cell system was utilized. Gels were placed into the electrophoresis chamber and filled with electrophoresis buffer. Depending on the protein that should be analyzed, 15 - $20 \mu \mathrm{g}$ protein were loaded into the gel pockets. Initially, electrophoresis was carried out at $50 \mathrm{~V}$ for $20 \mathrm{~min}$ to allow samples to enter the stacking gel. This was followed by electrophoresis at $100 \mathrm{~V}$ till the smallest proteins were reaching the end of the resolving gel. As a marker for molecular weight of the proteins BIO-RAD Precision Plus Dual Color Standard was used.

\subsubsection{Immunoblotting}

After electrophoresis the proteins were transferred to a PVDC membrane using the BIO-RAD Mini-PROTEAN Tetra Cell blotting system. To this, the membrane was activated in methanol for $30 \mathrm{sec}$ and placed next to the gel between two sheets of Whatman filter paper and sponge pads that were soaked in transfer buffer. The blotting cassette was inserted in the blotting system that was filled with ice cold transfer buffer. Protein transfer was performed by application of a current at constant voltage of $25 \mathrm{~V}$ overnight at $4^{\circ} \mathrm{C}$. After transfer, the membrane was incubated in blocking solution containing $5 \%$ non-fat milk in TBS-T for $60-90$ min at RT. Afterwards the membrane was rinsed 3 times for 10 min each and incubated with the primary antibody in TBS-T containing $2 \% \mathrm{BSA}$ over night at $4^{\circ} \mathrm{C}$. Subsequently, the membrane was rinsed 3 times in TBS-T for 10 min each and the secondary, horseradish peroxidase (HRP) coupled, goat anti-rabbit or goat anti-mouse antibody diluted in $1 \%$ non-fat milk in TBS-T was applied 
for $1 \mathrm{~h}$ at RT. The membrane was then washed 4 times for 10 min in TBS-T. For detection of protein bands, the membrane was incubated with a reagent for enhanced chemiluminescence (ECL) and the signal was produced by the HRP coupled to the secondary antibody. ECL-1 and ECL-2 were mixed in equal parts for at least 1 min directly before incubation on the membrane. The membrane was then exposed to autoradiography films for different times between 1 and $120 \mathrm{sec}$ using an autoradiography cassette and developed in a Curix 60 Developer.

\subsubsection{Neurochemical analysis of dopamine and dopamine metabolites in the murine striatum}

In order to investigate the functionality of dopaminergic terminals in the striatum, the content of dopamine (DA) and its metabolites homovanillic acid (HVA) and 3,4dihydroxyphenylacetic acid (DOPAC) were analyzed using high performance liquid chromatography (HPLC). To this, animals were sacrificed by cervical dislocation to exclude potential effects of anesthetic additives and allow immediate processing of the brain tissue. For isolation of the striatum, the brain was placed on an ice-cold surface, the two hemispheres were separated along the midline, and the right striatum was dissected. The tissue was transferred into $2 \mathrm{ml}$ cryovials containing $0.6-0.8 \mathrm{~g}$ of $1.4 \mathrm{~mm}$ Precellys ceramic beads and snap frozen in liquid nitrogen. $50 \mu \mathrm{l} 0.1 \mathrm{M} \mathrm{HClO}_{4}$ per mg striatal tissue were added to prevent enzymatic action and samples were homogenized using a bead mill homogenizer. Afterwards vials were centrifuged at 12.000 rounds per minute (rpm) at $4^{\circ} \mathrm{C}$ for $5 \mathrm{~min}$, the supernatant was transferred into a new tube and centrifuged at $13.000 \mathrm{rpm}$ at $4^{\circ} \mathrm{C}$ for $10 \mathrm{~min}$. For analysis of DA, HVA and DOPAC amounts, $50 \mu \mathrm{l}$ supernatant were transferred to HPLC vials and analyzed by HPLC.

The HPLC was performed in a system composed of a Guard cell, that was kept at $600 \mathrm{mV}$ to oxidize impurities in the eluent, a C18 reverse-phase HR-80 catecholamine column and an ESA Coulochem II electrochemical detector equipped with a 5011A analytic model detector $(\mathrm{E} 1=50 \mathrm{mV}, \mathrm{E} 2=400 \mathrm{mV}$ ). For sample loading the HPLC autosampler was cooled down to $6^{\circ} \mathrm{C}$ and samples were loaded. The autosampler automatically injected $20 \mu \mathrm{l}$ of each sample to the column. As mobile phase filtered and 
degased HPLC buffer running with a constant flow rate of $0.4 \mathrm{ml}$ per minute was used. During reverse-phase HPLC with ionizable substances it is important that the buffer is creating a stable $\mathrm{pH}$ to avoid changes in retention time and consequently in the chromatogram. Dopamine, HVA and DOPAC standards were run every 15 samples to allow an absolute measurement of the catecholamines in the samples. In order to prepare the standards, $10 \mu \mathrm{M}$ stock solutions were diluted with $0.1 \mathrm{M} \mathrm{HClO}_{4}$ to final concentrations of $0.15,0.3$ and $1.5 \mu \mathrm{M}$. Data was collected and processed by a Chromeleon Chromatography Data System. Analysis was performed by integrating the area under the specific peak curve and given in ng per mg wet tissue.

\subsubsection{Immunocytochemistry (ICC)}

In order to identify dopaminergic neurons in the primary midbrain neuron culture an immunolabeling against the dopaminergic marker tyrosine hydroxylase (TH) was performed. After fixation the coverslips were incubated for 10 minutes in $100 \mathrm{mM}$ ammoniumchloride in PBS to quench autofluorescence. Afterwards the coverslips were washed 3 times with PBS, followed by a permeabilization and blocking step for $10 \mathrm{~min}$ in Dako Antibody Diluent. After blocking, the coverslips were incubated with the primary polyclonal rabbit anti-tyrosine hydroxylase antibody (dilution: 1:1000 in Dako Antibody Diluent) for 90 minutes at RT. Subsequently, the coverslips were rinsed 3 times with PBS for 5 minutes each. Then the secondary Cy3-labeled goat anti-rabbit antibody (dilution: 1:500 in Dako Antibody Diluent) was applied and incubated for $30 \mathrm{~min}$ at $37^{\circ} \mathrm{C}$. After removing the antibody solution cells were washed 3 times in PBS and nuclear staining was performed using DAPI-solution for 5 minutes. After removing the DAPI-solution, coverslips were rinsed 3 times in PBS and mounted with Mowiol on microscope slides.

\subsubsection{Immunohistochemistry (IHC)}

In order to demonstrate transfection of dopaminergic neurons in the substantia nigra (SN) of mice with fluorescent NC siRNA $30 \mu \mathrm{m}$ thick coronal free floating sections of the midbrain were stained against TH. After washing the sections 3 times in PBS-T, 
unspecific antibody binding was blocked with $10 \%$ normal goat serum (NGS) in PBS-T for 30 min at RT. Afterwards sections were incubated with a primary rabbit anti-TH antibody diluted 1:1000 in $1 \% \mathrm{NGS}$ in PBS-T for $24 \mathrm{~h}$ at $4^{\circ} \mathrm{C}$ in order to label dopaminergic neurons. After removing the antibody solution sections were rinsed 3 times in PBS-T and incubated with a secondary goat anti-rabbit AlexaFluor 488 or DyLight 488 labeled antibody diluted 1:250 in PBS-T for $2 \mathrm{~h}$ at RT. Subsequently, the sections were rinsed 3 times with PBS-T and incubated with DAPI solution for $1 \mathrm{~min}$ for nuclear staining. After nuclear staining the sections were washed 3 times in PBS and collected on SuperFrost Plus microscope slides. After drying the sections at RT they were mounted with Mowiol.

To analyze the effect of the transfection reagent on microglial invasion of the substantia nigra after stereotactical injection of small RNAs, immunolabeling against the microglial marker lba1 was performed using the same protocol as described above, but with a primary rabbit anti-Iba1 antibody diluted 1:500 in $1 \%$ NGS in PBS-T.

In order to stereologically evaluate dopaminergic cell numbers in the SN, free floating sections were stained against $\mathrm{TH}$ followed by a Nissl staining. Sections were rinsed 3 times in TBS and endogenous peroxidases were quenched by incubation in $40 \%$ methanol and $1 \% \mathrm{H}_{2} \mathrm{O}_{2}$ in TBS for $15 \mathrm{~min}$. Afterwards sections were washed again in TBS and unspecific antibody binding was blocked with $10 \%$ NGS in TBS for 30 min at RT. Then the sections were incubated with a primary rabbit anti-TH antibody diluted 1:1000 in $1 \%$ NGS in TBS for $24 \mathrm{~h}$ at $4^{\circ} \mathrm{C}$. After removing the antibody solution the sections were washed 3 times in TBS, followed by incubation with a secondary biotinylated goat antirabbit antibody diluted 1:200 in TBS at RT for $2 \mathrm{~h}$ and treatment with the VECTASTAIN Elite $A B C$ Peroxidase Standard kit for $2 \mathrm{~h}$ at RT to enhance the signal. Afterwards sections were washed again 3 times for $5 \mathrm{~min}$ in TBS and the staining was visualized using the chromogen DAB (DAB Peroxidase Substrate kit) for $5 \mathrm{~min}$. The reaction was stopped by application of distilled water and sections were collected on SuperFrost Plus microscope slides. Slides were allowed to dry at RT and subsequently a Nissl staining was performed. To this, sections were rehydrated in distilled water for $5 \mathrm{~min}$ and incubated in thionine acetate for $7 \mathrm{~min}$, followed by a 2 min washing step in distilled water. Afterwards the tissue was dehydrated by incubation in ascending alcoholic solutions (70\%, $90 \%$ and 95 $\%$ ethanol) for $2 \mathrm{~min}$ each, and subsequent incubation in $100 \%$ isopropanol for $5 \mathrm{~min}$ and xylene 3 times for 5 min each. The moist sections were mounted with DPX. 
For analyses of striatal dopaminergic fiber density free floating sections were stained according to the DAB protocol described above for dopamine transporter (DAT), with the addition of nickel intensifier to increase the signal. There was no Nissl staining performed.

\subsubsection{Microscopy}

\subsubsection{Imaging and analysis of primary midbrain neuron cultures}

For investigation of dopaminergic neurite length, neuronal survival and neurite regeneration primary midbrain cultures on glass coverslips immunolabeled against $\mathrm{TH}$ were analyzed. To this, coverslips were imaged with a Zeiss Axioplan microscope equipped with a 16-bit greyscale CCD camera using the 20x objective and AxioVision SE64 4.9.1 Software with the MosaiX module. Per coverslip two $2.1 \mathrm{~mm}^{2}$ large areas were randomly imaged and analyzed. All images in one experiment were acquired with the same exposure time and for analyses the same color intensities were used. Images were analyzed using ImageJ 1.48 software. For analyses of dopaminergic neurite length and neurite regeneration the NeuronJ macro was applied and for counting of dopaminergic cell bodies in the MPP${ }^{+}$experiments the CellCounter tool was used.

\subsubsection{Stereological quantification of microglial invasion in the $\mathrm{SN}$ of mice}

In order to investigate the effect of HiPerfect transfection reagent on microglial invasion after stereotactical injection of small RNAs in the murine SN the number of Iba1immunoreactive (IR) cells within a diameter of $330 \mu \mathrm{m}$ around the injection side was assessed. To this, every $4^{\text {th }}$ section was analyzed stereologically using Stereo Investigator software on an Axioplan microscope equipped with a MBF Q-imagin camera. At 5x object magnification a circle with a diameter of $330 \mu \mathrm{m}$ with the injection side as center point was outlined and Iba1-positive cells were counted at 40x objective magnification using the optical fractionator function of the Stereo Investigator software. Cells were counted 
in frames sized $50 \times 50 \mu \mathrm{m}$ and the total number of Iba1-positive cells was extrapolated by the optical fractionator for the whole area of interest over all analyzed slides.

\subsubsection{Stereological quantification of dopaminergic neurons in the murine SN}

In order to analyze the number of dopaminergic (TH-positive) neurons and the total number of neurons (Nissl-positive cells) in the SN every $4^{\text {th }}$ DAB stained section over the whole SN was counted using Stereo Investigator software on an Axioplan microscope equipped with a MBF Q-imagin camera. At 2.5x objective magnification the SN was manually outlined and the Nissl-positive and TH-positive cells were counted at 40x objective magnification in counting frames sized $50 \times 50 \mu \mathrm{m}$ as described above for the whole unilateral SN per animal. The number of Nissl-positive cells was evaluated to exclude a mere downregulation of TH-expression and confirm a real loss of neurons.

\subsubsection{Measurement of the dopaminergic fiber density in mouse striata}

To analyze the density of striatal dopaminergic fibers frontal sections between bregma +0.62 and $-0.10 \mathrm{~mm}$ were stained against TH and DAT. All sections of one animal were imaged using an Axioplan microscope equipped with a 16-bit greyscale CCD camera using the $2.5 \times$ objective and AxioVision SE64 4.9.1 Software with the MosaiX module. The mean grey value of the striatum and cortex were measured using Image 1.48 with the freehand selection tool and further processing with background subtraction. Five sections per animal were analyzed and the mean TH-positive / DAT-positive signal was calculated in relation to the TH-positive signal / DAT-positive of the respective controls.

\subsubsection{Statistical analyses}

For statistical analysis Kyplot software (Version 2.0, KyensLab Incorporated, Tokyo, Japan) and SigmaPlot software (Version 11, Systat Software GmbH, Erkrath, Germany) were used. Parametric statistical methods were applied, as considered valid for most 
biological samples (Zar, J.H., Biostatistical Analysis, $4^{\text {th }}$ Edition. Prentice Hall, Upper Saddle River, NJ, US. 1999). For group comparisons, one-way ANOVA with Dunnet's post-hoc test was applied. The statistical tests and the number of experiments or animals used for analysis are indicated in the respective figure legends. Data are given as mean \pm SEM. Differences were considered significant with $p<0.05$ (* $p<0.05$; ** $p<0.01$; *** $p<$ 0.001). 


\section{Results}

\section{1 miRNAs in maturation and neurite outgrowth of primary midbrain neurons} (PMN) - Providing tools for neuronal protection and regeneration?

In order to analyze differences in miRNA expression levels upon maturation and neurite outgrowth of primary midbrain neurons (PMN) a massive parallel sequencing of small non-coding RNAs was performed. It was assumed that the cellular mechanisms that drive maturation and neurite outgrowth during development are similar to those that might enhance neuroprotection and regeneration after neuronal lesion. Detailed knowledge and understanding about the changes in miRNA levels during development of PMNs could provide new therapeutic strategies for the treatment of neurodegenerative diseases like PD, where these neurons degenerate. In addition to that, the effect of the pro-dopaminergic growth factor GDNF on miRNA expression in developing PMNs was analyzed.

\subsection{1 miRNA expression levels in developing PMNs - Major changes occur during early development}

For comparison of miRNA expression levels in developing PMNs total RNA was isolated at three different time points in vitro: At day 1 in vitro (DIV 1), which is shortly before or after the neurons become post-mitotic, at DIV 5, which represents an intermediate time point, and at DIV 10, were the PMN culture is mature (Figure 3.1/1 a). Cultures were partially treated with GDNF to analyze the effect of this pro-dopaminergic growth factor on miRNA expression levels. Massive parallel sequencing of small RNA libraries and differential expression analysis were performed.

Massive parallel sequencing of PMN small RNA libraries derived between 5 and 15 million reads per condition. From the total 1410 known miRNAs encoded in the mouse genome, 848 were detected in PMN cultures. Figure 3.1/1 b shows the proportion of sequence counts per miRNA with respect to the total number of counts attributed to miRNAs in PMNs at the respective maturation state. PMNs at DIV 1 (left panel) show high expression levels of miR-92a and miR-92b (indicated in red). 
a

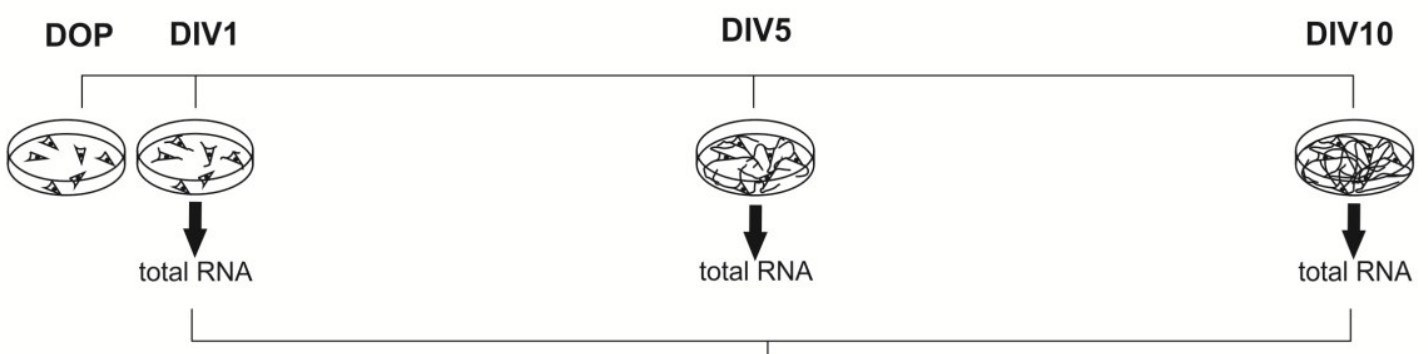

Small RNA Sequencing

b

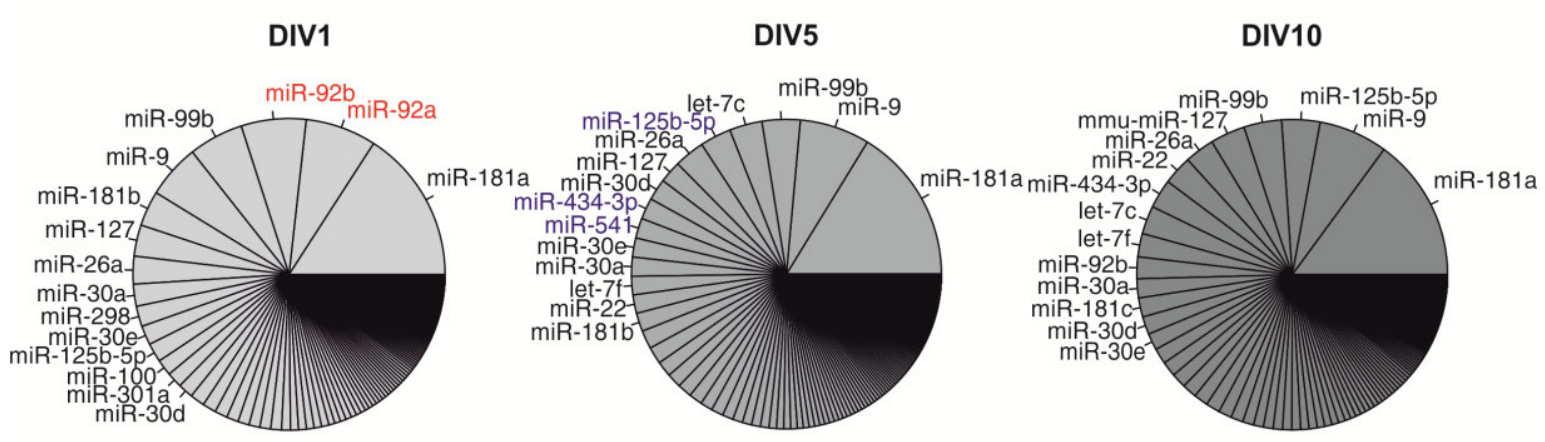

C
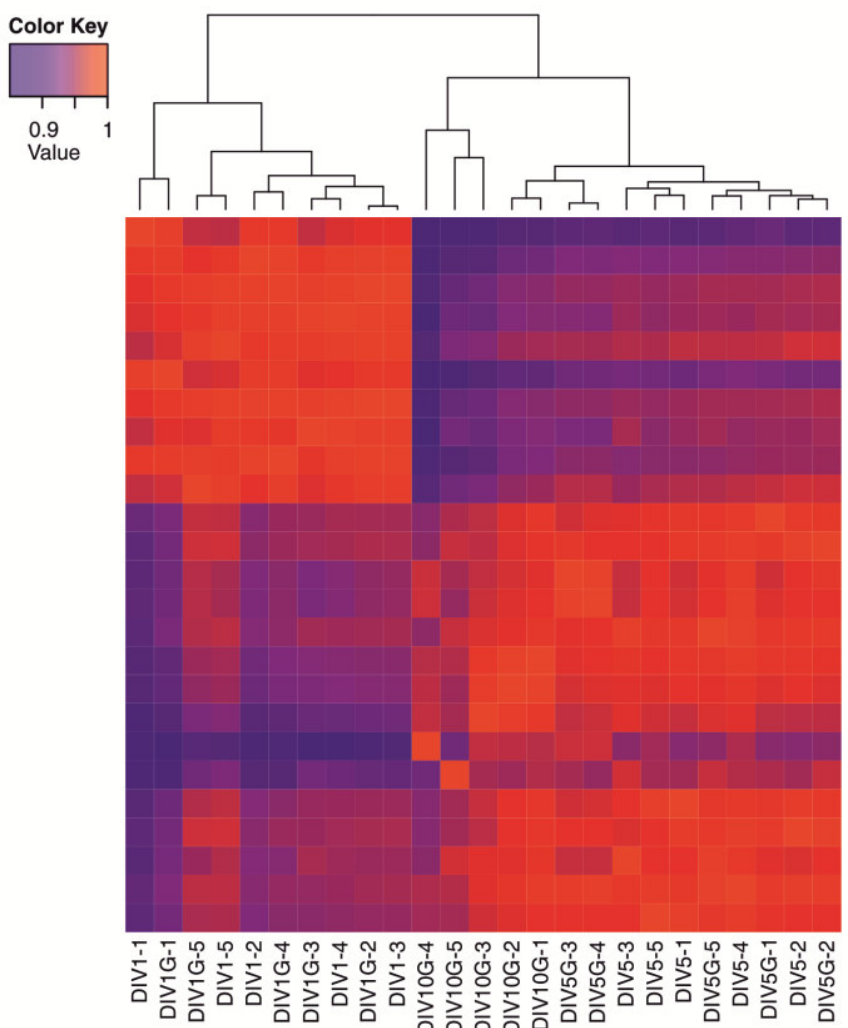

d

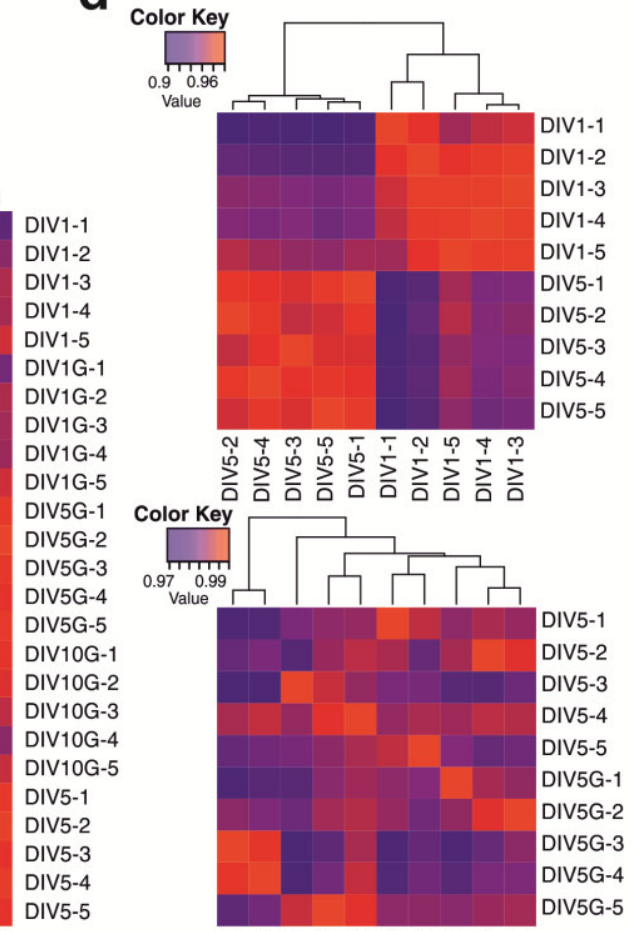

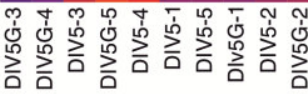

Figure 3.1/1: Small RNA sequencing reveals changes in the miRNAome of PMNs during maturation. (a) Experimental layout. Total RNA was isolated at day 1, day 5 and day 10 in vitro (DIV) (DOP = day of preparation). Additionally, cultures were treated with GDNF. (b) Proportion of sequence counts per miRNA with respect to the total number of counts attributed to miRNAs in PMNs at the respective maturation state. Red miRNA names indicate miRNAs expressed preferentially in neuronal precursors, blue miRNA names indicate miRNAs involved in differentiation and neurite 
growth. (c) Plot correlation displaying results of the differential expression analysis for all samples ( $n=5$ per condition). (d) Top panel: Plot correlation for the differential expression analysis comparing the miRNAomes of PMNs at DIV1 and DIV5. Bottom panel: Results for the differential expression analysis investigating the effect of GDNF treatment at DIV5.

These miRNAs are highly expressed in neuronal progenitor cells and predicted to maintain the progenitor state (Bian et al. 2013; Nielsen et al. 2009).

In comparison to this, at DIV 5 (middle panel) miR-92a and miR-92b are less expressed. Instead, several miRNAs known to be enriched in neurons (indicated in blue) are expressed at higher levels, e.g. miR-434-3p and miR-541 (Jovicic et al. 2013). The comparison between the miRNAomes at DIV 5 and DIV 10 (right panel) shows that PMNs at both time points have similar expression levels of neuron specific miRNAs.

The results for the differential expression analysis between all samples are shown in the correlation plot in Figure 3.1/1 c. It revealed that the major changes in miRNA expression occur during early development of PMNs, between DIV 1 and DIV 5, reflecting the already visible changes in the miRNAomes of PMNs at these time points as described above. The plot shows that samples are clearly clustered into two groups. One group contains all samples from DIV 1, independent if treated with GDNF or not. The second group contains all samples from DIV 5 and DIV 10. The upper panel of figure $3.1 / 1 \mathrm{~d}$ shows the plot correlation between samples of DIV 1 and DIV 5, the two conditions are notably clustered into two different groups, indicating strong differences in their miRNA expression profiles. The lower panel of figure 3.1/1 d displays the correlation plot for the comparison of DIV 5 samples treated with GDNF and DIV 5 samples that did not receive treatment with the pro-dopaminergic growth factor. Those samples are not clustered into different groups, but form a single cluster, indicating no major differences in miRNA expression patterns. These results show that the major changes in miRNA expression of PMNs occur between DIV 1 and DIV 5. Furthermore it becomes clear that GDNF leads only to minor changes in the expression of single miRNAs (see 3.1.2).

The comparison between miRNA expression patterns in PMNs at DIV 1 and DIV 5 revealed 163 miRNAs with significantly regulated expression levels. This equals a proportion of $19.62 \%$ (see figure $3.1 / 2$ a). Of the 15 most abundant miRNAs miR-434-3p was significantly upregulated at DIV 5, whereas miR-92a showed a significant decrease in expression at this time point. 
a

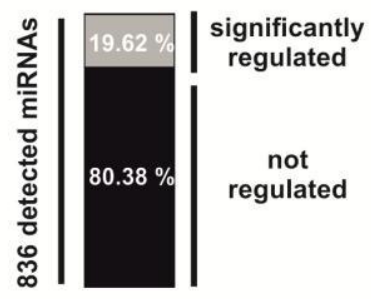

b

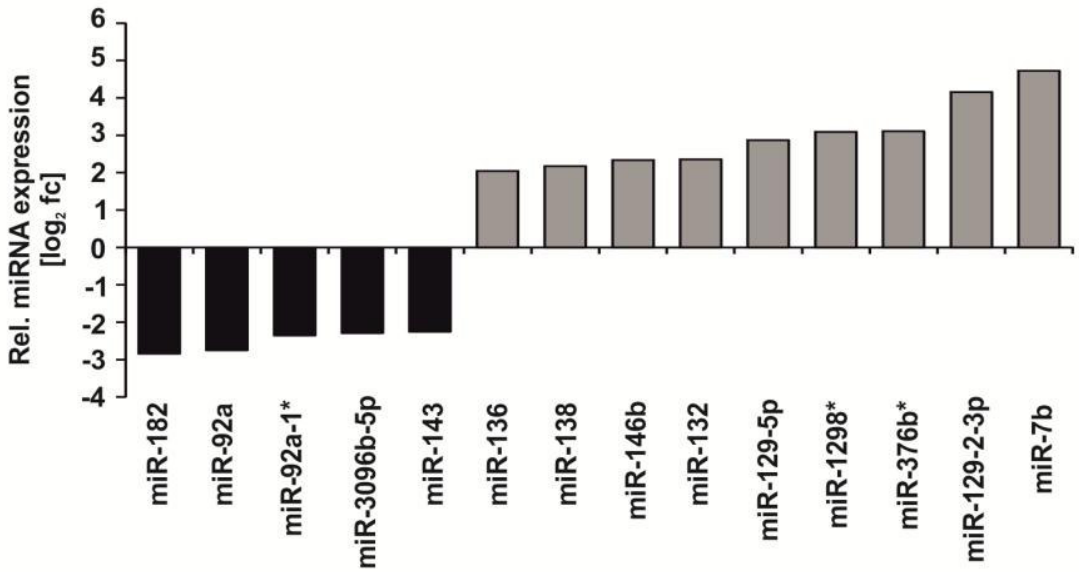

C Downregulated miRNAs

\begin{tabular}{ccc}
$\#$ & GO: Biological process & p-value \\
\hline 1 & Cellular process & $2.90 \mathrm{E}-28$ \\
2 & $\begin{array}{c}\text { Developmental process } \\
\text { Multicellular organismal } \\
\text { development }\end{array}$ & $2.20 \mathrm{E}-23$ \\
& $1.70 \mathrm{E}-22$ \\
4 & $\begin{array}{c}\text { Anatomical structure } \\
\text { morphogenesis }\end{array}$ & $2.19 \mathrm{E}-22$ \\
5 & Nervous system development & $5.20 \mathrm{E}-22$
\end{tabular}

\begin{tabular}{|c|c|c|}
\hline \# & $\begin{array}{c}\text { GO: Panther database: } \\
\text { Biological process }\end{array}$ & p-value \\
\hline 1 & BP00199:Neurogenesis & $2.60 \mathrm{E}-14$ \\
\hline 2 & $\begin{array}{l}\text { BP00246: Ectoderm } \\
\text { development }\end{array}$ & $9.40 \mathrm{E}-13$ \\
\hline 3 & $\begin{array}{l}\text { BP00111:Intracellular signaling } \\
\text { cascade }\end{array}$ & $1.00 \mathrm{E}-08$ \\
\hline 4 & $\begin{array}{c}\text { BP00285:Cell structure and } \\
\text { motility }\end{array}$ & $1.30 \mathrm{E}-08$ \\
\hline 5 & BP00040:mRNA transcription & $1.90 \mathrm{E}-08$ \\
\hline
\end{tabular}

\section{Upregulated miRNAs}

\begin{tabular}{|c|c|c|}
\hline \# & GO: Biological process & p-value \\
\hline 1 & Regulation of $\mathrm{m}$ & \\
\hline 2 & Regulation of gen & $3.10 \mathrm{E}-35$ \\
\hline 3 & $\begin{array}{l}\text { Regulation of macromolecule } \\
\text { biosvnthetic process }\end{array}$ & 4 १९ \\
\hline 4 & $\begin{array}{c}\text { Regulation of primary metabolic } \\
\text { process }\end{array}$ & $2.10 \mathrm{E}-3$ \\
\hline 5 & $\begin{array}{c}\text { Regulation of cellular metabolic } \\
\text { process }\end{array}$ & 3.4 \\
\hline
\end{tabular}

\begin{tabular}{ccc}
$\#$ & $\begin{array}{c}\text { GO: Panther database: } \\
\text { Biological process }\end{array}$ & p-value \\
\hline 1 & $\begin{array}{c}\text { BP00040:mRNA transcription } \\
\text { BP00044:mRNA transcription } \\
\text { regulation }\end{array}$ & $6.20 \mathrm{E}-20$ \\
2 & $\begin{array}{c}9.10 \mathrm{E}-16 \\
\text { BP00031:Nucleoside, nucleotide } \\
\text { and nucleic acid metabolism }\end{array}$ & $4.20 \mathrm{E}-15$ \\
4 & BP00199:Neurogenesis & $5.70 \mathrm{E}-14$ \\
5 & BP00246:Ectoderm development & $7.30 \mathrm{E}-14$
\end{tabular}

\begin{tabular}{ccc}
$\#$ & KEGG pathway & p-value \\
\hline 1 & Axon guidance & $1.30 \mathrm{E}-17$ \\
2 & Prostate cancer & $1.63 \mathrm{E}-12$ \\
3 & Acute myeloid leukemia & $2.13 \mathrm{E}-12$ \\
4 & Dorso-ventral axis formation & $1.07 \mathrm{E}-09$ \\
5 & Wnt signaling pathway & $2.27 \mathrm{E}-09$
\end{tabular}

Figure 3.1/2: Massive regulation of miRNA expression during maturation of PMNs and functional analysis of regulated miRNAs. (a) Proportion of regulated miRNAs among all miRNAs detected in PMNs. (b) Highly regulated miRNAs ( $\geq 4$ fold change in expression) with on average more than 500 reads per condition upon maturation and neurite outgrowth in PMNs. (c) Functional analysis of gene targets (TargetScan mouse 6.2) incorporating upregulated or 
downregulated miRNA seeds. Enrichment of gene ontology (GO) terms for biological processes as well as biological process terms from the PANTHER database and KEGG pathways were analyzed.

Out of the 163 significantly regulated miRNAs 47 were very strongly regulated ( $\geq 4$ fold), thereof the 14 most abundant miRNAs are displayed in figure $3.1 / 2 \mathrm{~b}$ with the relative expression levels given in $\log _{2}$ fold change.

In order to analyze the biological functions of these miRNAs, target prediction analyses were performed. Subsequently, the target gene lists of upregulated and downregulated miRNAs were combined, respectively, and a functional annotation to biological processes, biological process terms from the PANTHER database and KEGG pathways was performed. The results of these analyses are displayed in figure $3.1 / 2 \mathrm{c}$, showing the top five processes and pathways in which the miRNA target genes are involved. The left panel shows the results for the target genes of the downregulated miRNAs and the right panel for the upregulated miRNAs. According to the functional annotation data the target genes of upregulated as well as downregulated miRNAs are involved in key processes important for neuronal development. For the functional annotation of target genes of downregulated miRNAs the terms 'nervous system development', 'neurogenesis' and 'neurotrophin signaling pathway' were among the top five processes, indicating that changes in the expression of miRNAs targeting these genes have a high impact on the mentioned processes. In addition to this, more general terms like 'developmental process', 'cell structure and motility' as well as 'PI3K-Akt signaling pathway' were also listed, reflecting the impact of miRNA expression changes on cellular physiology and metabolism.

The functional annotation of target genes of upregulated miRNAs showed the terms 'axon guidance' and 'neurogenesis' as directly neuron-related terms, displaying the effect of expression changes of the listed miRNAs on neuronal development. Furthermore, the target genes of the upregulated miRNAs are involved in regulation of gene expression and transcription, as shown by the terms 'regulation of gene expression', 'mRNA transcription' and 'mRNA transcription regulation'.

In addition to these combined analyses, individual functional annotation analyses of the target genes of the above mentioned 14 miRNAs were performed in order to find 
miRNA candidates with a neuroprotective potential or influence on neurite development. As these results do not play a continuative role in this thesis they are not shown here.

\subsubsection{GDNF treatment lead to specific changes in the miRNAome of PMNs - Only miR-182 and miR-183 are significantly regulated}

In order to analyze the effect of GDNF treatment on miRNA expression levels in PMN cultures, miRNA expression was compared between PMN cultures treated with GDNF and control-treated cultures at DIV 1, DIV 5 and DIV 10. In contrast to the massive changes in miRNA expression during development, GDNF treatment led to a very specific regulation of just two miRNAs, miR-182 and miR-183.

In figure 3.1/3 a the downregulation of miR-182 and miR-183 between DIV 1 and DIV 5 analyzed by small RNA sequencing is displayed. The sequencing showed an almost 3-fold (-2.84 $\left.\log _{2} \mathrm{fc}\right)$ downregulation for miR-182 and a nearly 2-fold (-1.95 $\log _{2} \mathrm{fc}$ ) downregulation for miR-183 in PMNs at DIV 5 compared to PMNs at DIV 1. In contrast to this decrease of miR-182 and miR-183 levels in untreated PMNs, the small RNA sequencing of libraries derived from PMNs treated with GDNF showed an increase in the expression of both miRNAs at DIV 5 (figure $3.1 / 3 \mathrm{~b}$ ). The expression levels of miR-182 at DIV 5 increased 3-fold (3.02 fc) upon GDNF treatment, whereas miR-183 expression increased a little more than 2-fold (2.10 fc).

In order to confirm the data derived by massive parallel small RNA sequencing and to obtain more insight into the regulation of miR-182 and miR-183 expression upon GDNF treatment, total RNA from either GDNF- or control-treated cultures was isolated at DIV 1, DIV 3, DIV 5 and DIV 7 and qRT-PCR to analyze miR-182 and miR-183 expression was performed. Figure $3.1 / 3 \mathrm{c}$ displays the results of these analyses. Already at DIV 1 GDNF treatment led to an increase in the expression of both miRNAs (miR-182: $1.8 \pm 0.2 \mathrm{fc}$; miR183: $2.0 \pm 0.2 \mathrm{fc}$ ), even though this increase was not statistically significant. At DIV $3 \mathrm{miR}$ $182(2.0 \pm 0.2 \mathrm{fc})$ as well as miR-183 (2.4 $\pm 0.1 \mathrm{fc})$ expression levels were significantly higher in PMN cultures treated with GDNF. In addition to this, the sequencing data showing that GDNF leads to an increased expression of those miRNAs at DIV 5 was confirmed. Quantitative RT-PCR showed that at DIV 5 miR-182 expression significantly 
increased 2-fold (2.0 $\pm 0.1 \mathrm{fc})$ and miR-183 expression showed a $1.8 \pm 0.2$ fold increase. Also at DIV 7 GDNF treated PMN cultures showed a significant increase in miR-182 (1.6 \pm $0.1 \mathrm{fc})$ and miR-183 (2.1 $\pm 0.2 \mathrm{fc})$ expression levels.

a

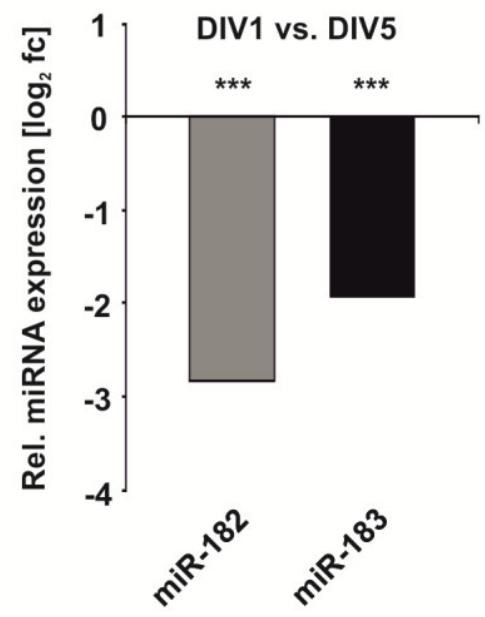

b

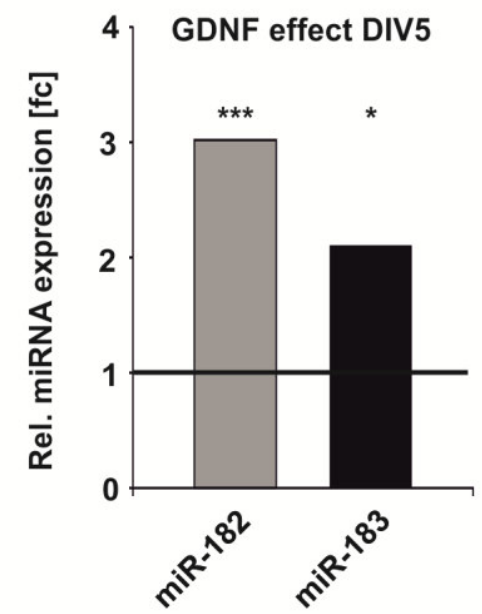

C

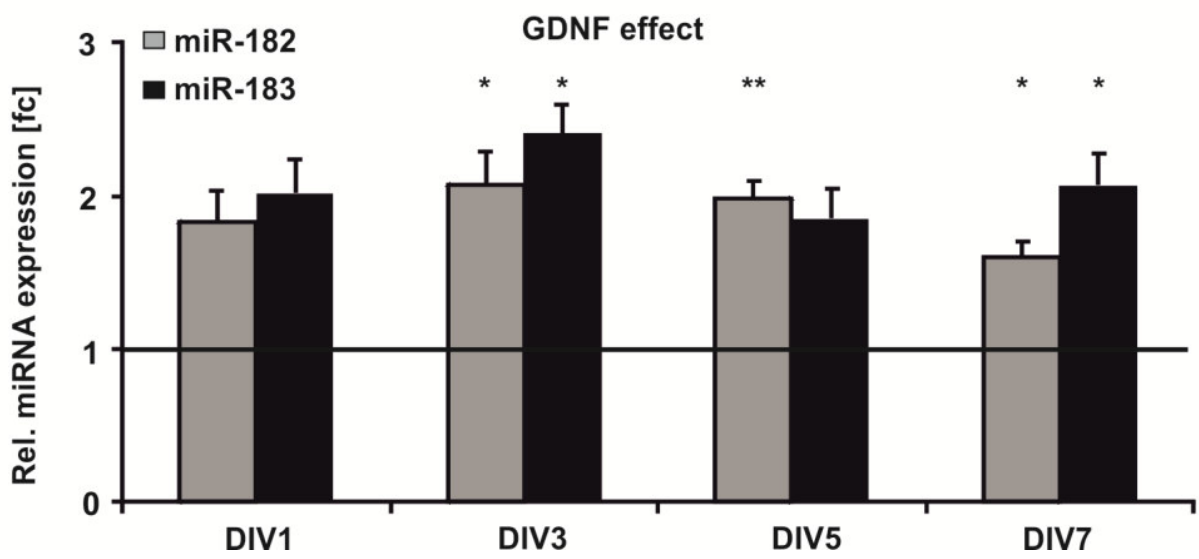

Figure 3.1/3: GDNF treatment of PMNs leads to a specific upregulation of miR-182 and miR-183. (a) Massive parallel sequencing of small RNA libraries shows a downregulation of miR-182 and miR-183 in PMNs upon maturation ( $\mathrm{n}=5$ ). (b) GDNF treatment of PMNs leads to a specific upregulation of miR-182 and miR-183 as revealed by small RNA sequencing. (c) qRT-PCR confirms upregulation of miR-182 and miR-183 in GDNF treated PMNs $(n=4)$.

\subsubsection{Functional annotation of miR-182 and miR-183 target genes - Potential role in dopaminergic neuron biology}

To gain a first insight in the role of miR-182 and miR-183 in dopaminergic neuron biology, a prediction analysis for genes targeted by miR-182 and miR-183 was performed. 
This was followed by the functional annotation of these genes to certain biological processes and KEGG-pathways.

In figure 3.1/4 a the results for the functional annotation analyses of miR-182 (left panel) and miR-183 (right panel) target genes are displayed, showing the top five terms for the gene ontology biological process analysis (top), the Panther database biological process terms (middle) and the KEGG pathway analysis (bottom).

a

Functional annotation of miR-182 targets

Functional annotation of miR-183 targets

\begin{tabular}{|c|c|c|c|c|c|}
\hline$\#$ & GO: Biological process & p-value & $\#$ & GO: Biological process & p-value \\
\hline 1 & Cell motion & $6.30 \mathrm{E}-10$ & 1 & Homophilic cell adhesion & $3.50 \mathrm{E}-09$ \\
\hline 2 & Cell projection organization & $3.50 \mathrm{E}-09$ & 2 & Cell-cell adhesion & $8.40 \mathrm{E}-07$ \\
\hline 3 & Neuron projection development & $5.90 \mathrm{E}-08$ & 3 & Cell adhesion & $6.70 \mathrm{E}-06$ \\
\hline 4 & $\begin{array}{l}\text { Cell morphogenesis involved in } \\
\text { neuron differentiation }\end{array}$ & $6.80 \mathrm{E}-08$ & 4 & Biological adhesion & $6.90 \mathrm{E}-06$ \\
\hline 5 & Neuron development & $1.20 \mathrm{E}-07$ & 5 & Phosphate metabolic process & $2.90 \mathrm{E}-05$ \\
\hline
\end{tabular}

\begin{tabular}{|c|c|c|c|c|c|}
\hline \# & $\begin{array}{l}\text { GO: Panther database: } \\
\text { Biological process }\end{array}$ & p-value & $\#$ & $\begin{array}{c}\text { GO: Panther database: } \\
\text { Biological process }\end{array}$ & p-value \\
\hline 1 & BP00199:Neurogenesis & 3.90E-09 & 1 & BP00124:Cell adhesion & 3.90E-09 \\
\hline 2 & $\begin{array}{l}\text { BP00246:Ectoderm } \\
\text { development }\end{array}$ & $5.20 \mathrm{E}-08$ & 2 & BP00242:Embryogenesis & $5.20 \mathrm{E}-08$ \\
\hline 3 & BP00063:Protein modification & $5.40 \mathrm{E}-07$ & 3 & $\begin{array}{l}\text { BP00064:Protein } \\
\text { phosphorvlation }\end{array}$ & $5.40 \mathrm{E}-07$ \\
\hline 4 & $\begin{array}{l}\text { BP00111:Intracellular signaling } \\
\text { cascade }\end{array}$ & $1.10 \mathrm{E}-06$ & 4 & $\begin{array}{l}\text { BP00285:Cell structure and } \\
\text { motility }\end{array}$ & $1.10 \mathrm{E}-06$ \\
\hline 5 & $\begin{array}{l}\text { BP00111:Intracellular signaling } \\
\text { cascade }\end{array}$ & $6.40 \mathrm{E}-06$ & 5 & $\begin{array}{l}\text { BP00120:Cell adhesion-mediated } \\
\text { signaling }\end{array}$ & $6.40 \mathrm{E}-06$ \\
\hline
\end{tabular}

\begin{tabular}{|c|c|c|c|c|c|}
\hline$\#$ & KEGG pathway & p-value & $\#$ & KEGG pathway & p-value \\
\hline 1 & Long-term depression & $8.51 \mathrm{E}-08$ & 1 & $\begin{array}{c}\text { Transcritional misregulation in } \\
\text { cancer }\end{array}$ & $5.13 \mathrm{E}-08$ \\
\hline 2 & Neurotrophin signaling pathway & $7.24 \mathrm{E}-06$ & 2 & Other glycan degradation & $1.38 \mathrm{E}-05$ \\
\hline 3 & $\begin{array}{l}\text { Bacterial invasion of epithelial } \\
\text { cells }\end{array}$ & 7.27E-06 & 3 & Wnt signaling pathway & $3.25 \mathrm{E}-05$ \\
\hline 4 & Oocyte meiosis & $5.06 \mathrm{E}-05$ & 4 & Dopaminergic synapse & $3.43 \mathrm{E}-05$ \\
\hline 5 & Long-term potentiation & $5.06 \mathrm{E}-05$ & 5 & Tight junction & $5.12 \mathrm{E}-09$ \\
\hline
\end{tabular}

Figure 3.1/4: Functional analysis of miR-182 (left panel) and miR-183 (right panel) target genes (TargetScan mouse 6.2). (a) Analyses of enrichment of gene ontology (GO) terms for biological processes as well as biological process terms from the PANTHER database and KEGG pathways. 
According to the gene ontology analysis, the target genes of miR-182 are involved in 'cell motion', 'cell projection organization', 'neuron projection development', 'cell morphogenesis involved in neuron development' and 'neuron development'. In addition to this the Panther and KEGG pathway analysis revealed terms like 'neurogenesis', 'intracellular signaling cascade', 'long-term depression' and 'long-term potentiation' as well as 'neurotrophin signaling pathway', the latter underlining the connection with GDNF. Taken together the functional annotation analyses of miR-182 target genes indicate a potential important role of this miR-182 in dopaminergic neuron development and differentiation.

For miR-183 target genes the gene ontology biological process analysis revealed terms like 'cell adhesion', 'cell-cell adhesion' and 'biological adhesion'. This is complemented by the result of the Panther database analysis, which showed terms like 'cell structure and motility' and 'cell-adhesion mediated signaling' as well as by the results of the KEGG pathway analysis, which revealed terms like 'dopaminergic synapse' and 'Wnt signaling pathway' among others. The functional annotation analyses of miR-183 target genes show that this miRNA also might have important functions in the developmental biology of dopaminergic neurons.

For a better understanding of the role of miR-182 and miR-183 in dopaminergic neurons these two miRNAs were subject of an extensive characterization in vitro and in vivo. The results of these experiments are described from chapter 3.3.1 on. 


\section{2 miRNA expression changes upon dopaminergic neuron degeneration and regeneration - Insights from the 6-OHDA mouse model for PD}

In order to analyze the role of miRNAs upon degeneration and regeneration of dopaminergic neurons in vivo, a massive parallel sequencing of small non-coding RNA libraries derived from SNpc containing midbrain samples of striatal 6-OHDA lesioned animals was performed. In addition one group of animals received daily physical training. Spontaneous regeneration in the nigrostriatal system of rodents after toxic impairment is well described (Höglinger et al. 2004; Stanic et al. 2003). Furthermore it is known that physical activity is neuroprotective and enhances neuroregeneration (Tillerson et al. 2003; Tillerson et al. 2002). Detailed knowledge about miRNAs involved in the degeneration and regeneration of the murine nigrostriatal system could help to gain further insight into the pathomechanism of dopaminergic neurodegeneration as well as to find potential new therapeutic targets that might enhance neuroregeneration or inhibit further degeneration of dopaminergic neurons.

\subsubsection{The miRNAome of the murine SNpc containing midbrain}

The animals received a stereotactic injection of $4 \mu \mathrm{g}$ 6-OHDA into their right striatum, leading to the progressive die-back of dopaminergic fibres and subsequently neuronal cell bodies in the SNpc. In order to analyze the changes in the miRNAome during degeneration, animals were sacrificed 4 weeks (degeneration model) after striatal injection, which is the time point when maximal degeneration occurred. For analysis of the miRNAome upon regeneration, animals were sacrificed 12 weeks (regeneration model) after the striatal 6-OHDA injection. At this time point the regenerative response begins (figure 3.2/1 a).

Small RNA sequencing of murine SNpc containing midbrain samples yielded between 3.3 and 21 million reads per condition. Among all small non-coding RNA reads 71.14 percent were belonging to mature miRNAs, whereas the other 28.86 percent were other small non-coding RNAs (figure 3.2/1 b). Out of 1410 known mature murine miRNAs, 780 were detected in the SNpc containing midbrain (figure 3.2/1 c). 
a

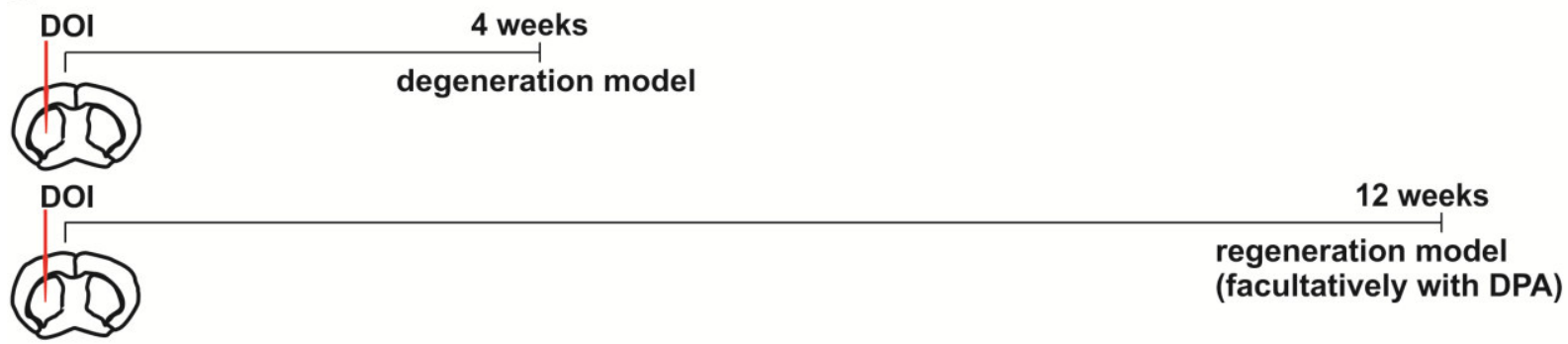

b

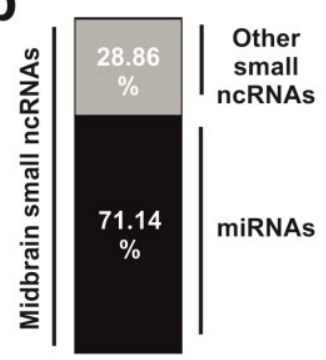

C

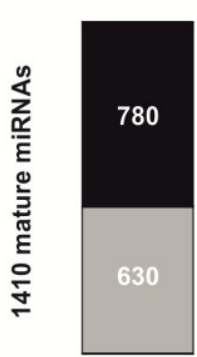

Detected in midbrain

$\square$ Not detected in midbrain d

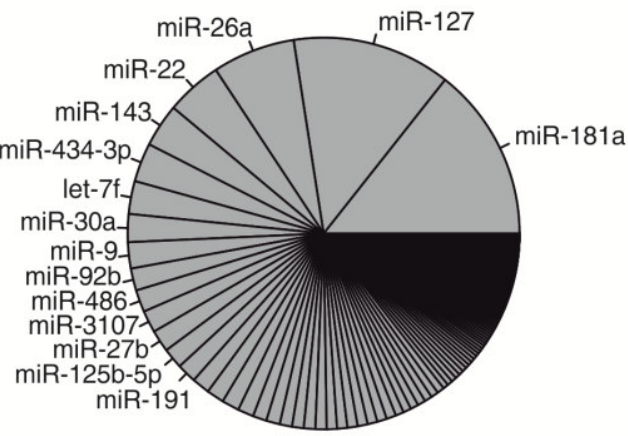

e
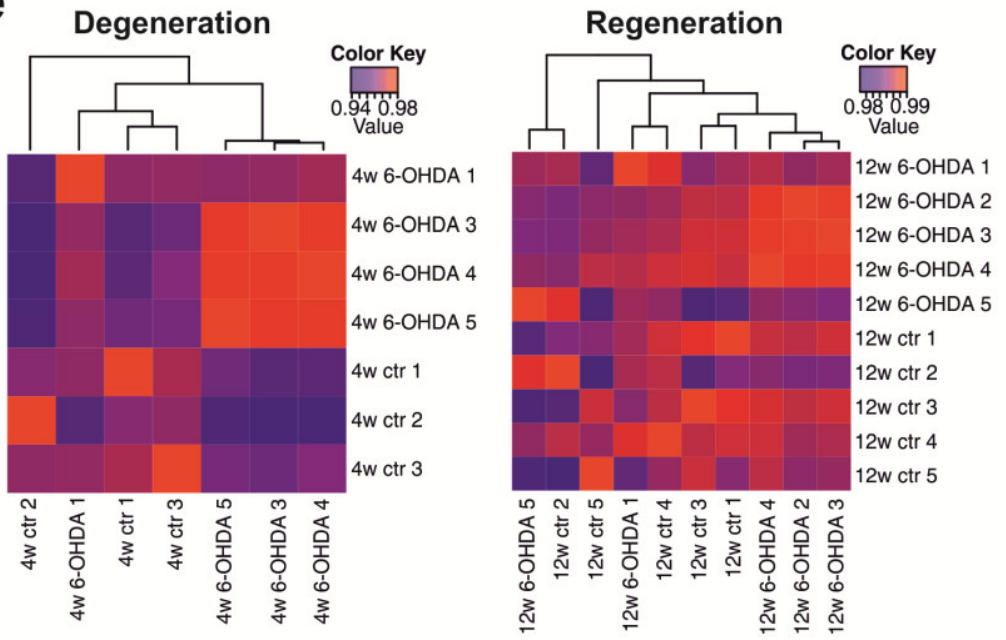

Physical Activity
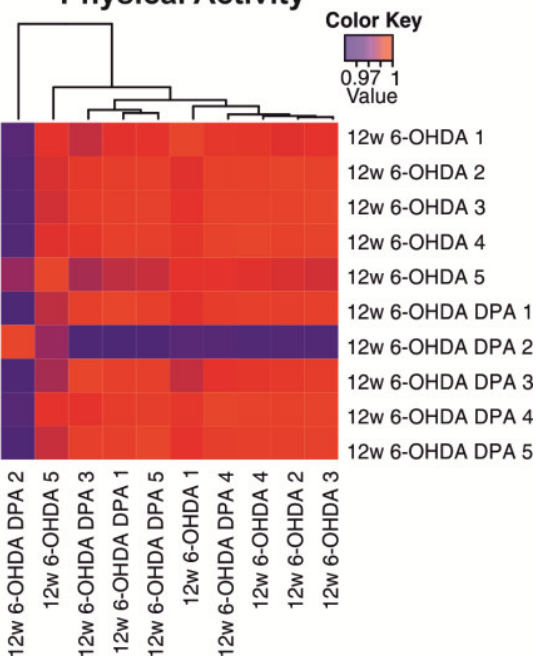

Figure 3.2/1: Small RNA sequencing of the midbrain in the 6-OHDA mouse model for PD. (a) Experimental layout for the 4 weeks post unilateral striatal 6-OHDA injection degeneration model and the 12 weeks post unilateral striatal 6OHDA injection regeneration model. (b) Contribution of miRNAs to the total number of small (18 - $26 \mathrm{nt}$ ) non conding RNAs detected in the murine midbrain by small RNA sequencing. (c) Proportion of detected mature miRNAs regarding the total number of known mature murine miRNAs in miRBase. (d) Proportion of sequence counts per miRNA with respect to total number of counts attributed to miRNAs in the untreated murine midbrain. (e) Left panel: Results of the differential expression analysis comparing the miRNAomes of untreated midbrains with the miRNAomes of midbrains of the 4 weeks 6-OHDA degeneration model. Middle panel: Plot correlation displaying the results of the differential expression analysis comparing the miRNAomes of untreated midbrain samples with the miRNAomes of midbrains 12 weeks after striatal 6-OHDA injection (regeneration model). Right panel: Plot correlation showing the results of the 
differential expression analysis comparing the midbrain miRNA expression 12 weeks after striatal 6-OHDA injection between animals receiving DPA or not ( $n=5$ animals per condition).

In figure 3.2/1 d the proportion of sequence counts per miRNA with respect to total number of counts attributed to miRNAs in the untreated murine midbrain is displayed. The most abundant miRNA in the murine brain is miR-9 (Chiang et al. 2010), in contrast to this, in the midbrain of unlesioned mice, brain-specific miR-181a showed the highest expression. Also other brain miRNAs as miR-127, miR-9, miR-99b and miR-125b are expressed at high levels.

\subsubsection{Changes in miRNA expression of the murine midbrain upon degeneration of the nigrostriatal system}

In order to analyze the changes in the miRNAome of the murine midbrain upon degeneration of the nigrostriatal tract, a differential expression analysis comparing the miRNAome of unlesioned animals with the miRNAome of degeneration model animals (4 weeks after striatal 6-OHDA injection) was performed. The plot correlation in figure 3.2/1 e (left panel) shows that miRNA expression was regulated 4 weeks after 6-OHDA lesion; with the exception of one sample the 4 weeks 6-OHDA samples are clustered into one group, whereas the samples from unlesioned samples are clustered in a second group.

The differential expression analysis revealed that out of 780 detected miRNAs the expression of 5 miRNAs was significantly changed in the degeneration model compared to unlesioned control animals. miR-501-3p, miR-100 and miR-331-3p were significantly upregulated during degeneration, whereas miR-486 and miR-3107 showed a decreased expression (figure 3.2/2 a). To understand which pathways are mainly affected by the differential expression of these miRNAs, a target prediction analysis followed by an annotation of these genes to KEGG pathways was performed. Figure 3.2/2 c (left panel) shows the top five KEGG pathways involving genes that are affected by those miRNAs. Among them are very general pathways important for neuronal differentiation, cellular function and survival as 'MAPK signaling pathway', 'Wnt signaling pathway' and 'mTOR signaling pathway' as well as 'axon guidance'. These results reflect the impact of 6-OHDA 
on the physiology of dopaminergic neurons and confirms the cellular mechanisms that are affected during neurodegeneration.

a

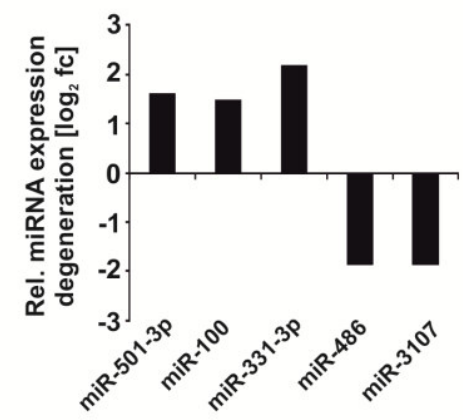

b

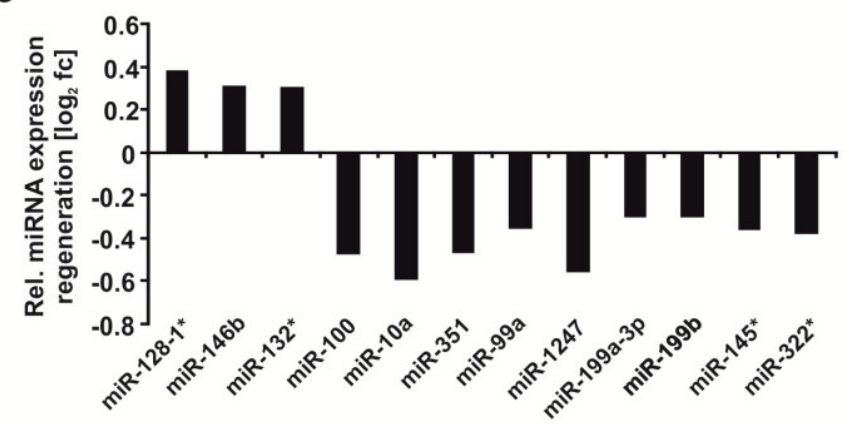

C Regulated miRNAs targets enrichment in KEGG pathways

\begin{tabular}{|c|c|c|c|c|c|}
\hline \multicolumn{3}{|c|}{ Degeneration } & \multicolumn{3}{|c|}{ Regeneration } \\
\hline$\#$ & KEGG pathway & p-value & $\#$ & KEGG pathway & p-value \\
\hline 1 & MAPK signaling pathway & $1.05 \mathrm{E}-05$ & 1 & Mucin type O-Glycan biosynthesis & $1.62 \mathrm{E}-14$ \\
\hline 2 & Wnt signaling pathway & $1.05 \mathrm{E}-05$ & 2 & ErbB signaling pathway & $7.48 \mathrm{E}-08$ \\
\hline 3 & mTOR signaling pathway & $1.64 \mathrm{E}-05$ & 3 & Glioma & $2.62 \mathrm{E}-06$ \\
\hline 4 & Axon guidance & $1.64 \mathrm{E}-05$ & 4 & Wnt signaling pathway & $3.37 \mathrm{E}-06$ \\
\hline 5 & Prostate cancer & $1.64 \mathrm{E}-05$ & 5 & Neurotrophin signaling pathway & $3.41 \mathrm{E}-05$ \\
\hline
\end{tabular}

Figure 3.2/2: Significantly regulated miRNAs in the murine midbrain 4 weeks (degeneration) and 12 weeks (regeneration) after striatal 6-OHDA lesion and functional analysis of their target genes. (a) Differentially expressed miRNAs in the murine midbrain 4 weeks after striatal 6-OHDA lesion (degeneration). (b) Significantly regulated miRNAs 12 weeks after striatal 6-OHDA lesion (regeneration). (c) Enrichment for KEGG pathways among miRNA target genes.

\subsection{3 miRNAs involved in spontaneous regeneration of SNpc dopaminergic neurons after 6-OHDA lesion}

In order to compare miRNA expression levels in the midbrain of unlesioned mice with animals starting spontaneous recovery (regeneration) 12 weeks after striatal 6OHDA lesion, a differential expression analysis was performed. In addition to this, the effect of daily physical training on miRNA expression was analyzed. 
The plot correlation in figure 3.2/1 e (middle panel) displays the results of the differential expression analysis comparing the miRNAome of unlesioned animals with the miRNAome of animals 12 weeks after 6-OHDA lesion (regeneration). There is no clustering of the samples in the two different conditions visible indicating that only minor changes in the miRNAome occur during regeneration. Out of 780 miRNAs detected in the murine SNpc containing midbrain by small RNA sequencing, 12 miRNAs were significantly changed in their expression. Compared to the expression changes upon degeneration of dopaminergic neurons, the changes are milder during regeneration. Three miRNAs were upregulated including miR-132*, miR-128-1* and miR-146b, whereas 9 miRNAs were downregulated during regeneration of the nigrostriatal tract. Among the downregulated miRNAs were miR-100, which is up regulated upon degeneration, miR-199a, miR-199b and miR-99a (figure $3.2 / 2$ b). Analysis of enrichment of KEGG pathways among miRNA target genes revealed that especially proteins involved in growth associated pathways as 'ErbB signaling pathway', 'glioma' and 'neurotrophin signaling pathway' are affected by the regulated miRNAs. This reflects the cellular mechanisms involved in a regenerative response.

In figure 3.2/1 e (right panel) the results of the differential expression analysis concerning the effect of physical activity on the miRNAome 12 weeks after 6-OHDA lesion are displayed. Compared to animals without additional daily training, there was no significant change in miRNA expression in the midbrain of trained animals. Thus, the effect of physical exercise seems not to be mediated by global changes in miRNA expression. 


\subsection{Transfection of GDNF-regulated miR-182 and miR-183 - Beneficial effects on dopaminergic PMNs in vitro?}

The previous experiments showed that GDNF treatment of PMNs resulted in an increase in miR-182 and miR-183. In order to analyze the effects of these miRNAs on dopaminergic PMNs, cultures were transfected with synthetic miR-182 and miR-183 mimics or a scrambled NC siRNA as control, which has no homology to any known mammalian gene and thus, even incorporated into RISC, has no effect on protein expression. The focus of this study was put on neurite length, neurite regeneration and neuroprotection in dopaminergic PMNs. Furthermore, the miRNA-mediated effects were compared to the effects of GDNF treatment.

\subsubsection{Increase in miR-182 and miR-183 leads to longer neurites in dopaminergic PMNs}

In order to find the optimal concentration of miRNA mimic for transfection of PMNs, cells were transfected at DIV 1 with different concentrations (100 pM, $5 \mathrm{nM}$ and $50 \mathrm{nM}$ ) of synthetic miRNA mimic. $24 \mathrm{~h}$ and $72 \mathrm{~h}$ later total RNA was isolated from transfected cultures and miR-182 and miR-183 levels were quantified by qRT-PCR. In figure 3.3/1 b the results of the qRT-PCR $24 \mathrm{~h}$ post-transfection are displayed. The left panel shows the miR-182 levels after transfection with indicated concentrations of miR182 mimic relative to expression levels in NC siRNA transfected cells given in $\log _{2}$ fold change and analyzed by the $\Delta \Delta$-Ct method. Even transfection with the lowest dose of 100 pM miR-182 mimic led to a strong increase in miR-182 levels $\left(9.6 \pm 0.1 \log _{2} \mathrm{fc}\right)$. Further increase in miR-182 mimic used for transfection led to additional dose dependent increase in miR-182 levels detected by qRT-PCR ( $5 \mathrm{nM}=12.9 \pm 0.7 \log _{2} \mathrm{fc} ; 50 \mathrm{nM}=13.9 \pm$ $0.1 \log _{2} \mathrm{fc}$ ). The right panel in figure $3.3 / 1 \mathrm{~b}$ shows the qRT-PCR results for PMNs transfected with different concentrations of miR-183 mimic $24 \mathrm{~h}$ post-transfection. Similar to PMNs transfected with miR-182, miR-183 transfection showed a dose dependent increase in miR-183 levels detected by qRT-PCR (100 pM $=8.6 \pm 0.3 \log _{2} \mathrm{fc} ; 5$ $\mathrm{nM}=12.0 \pm 0.6 \log _{2} \mathrm{fc} ; 50 \mathrm{nM}=14.1 \pm 0.5 \log _{2} \mathrm{fc}$ ). Increased miRNA levels were stable 
also $72 \mathrm{~h}$ post-transfection. For further analyses the $5 \mathrm{nM}$ dosage was chosen, as it had no toxic effects and guaranteed optimal transfection.

a

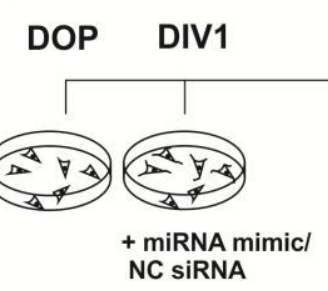

b

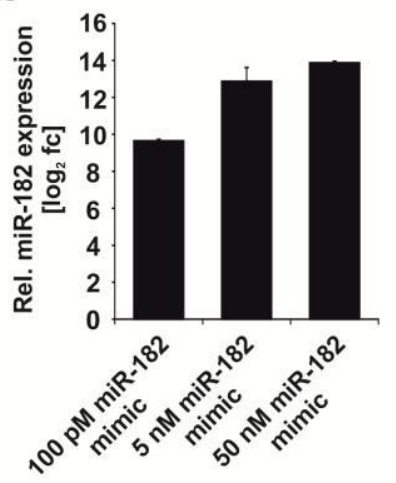

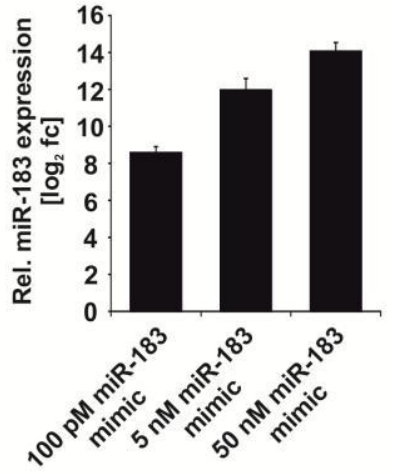

C

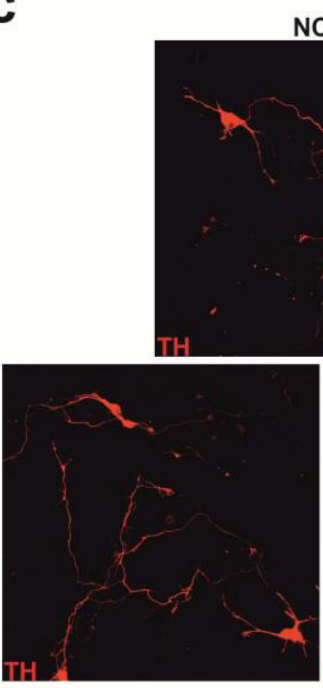

miR-182 mimic
NC
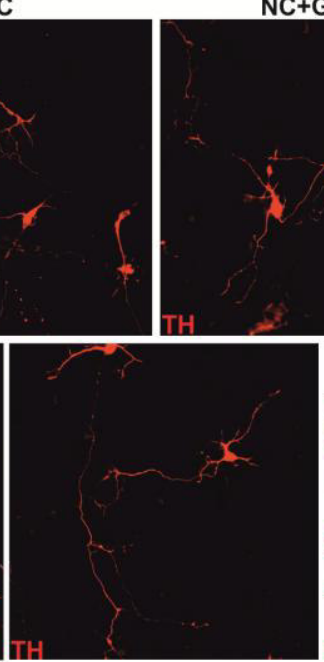

miR-183 mimic
NC+GDNF
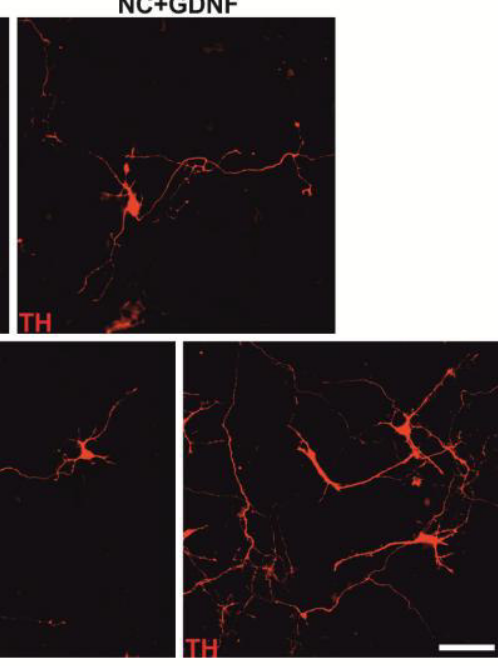

miR-182/ miR-183 mimic d

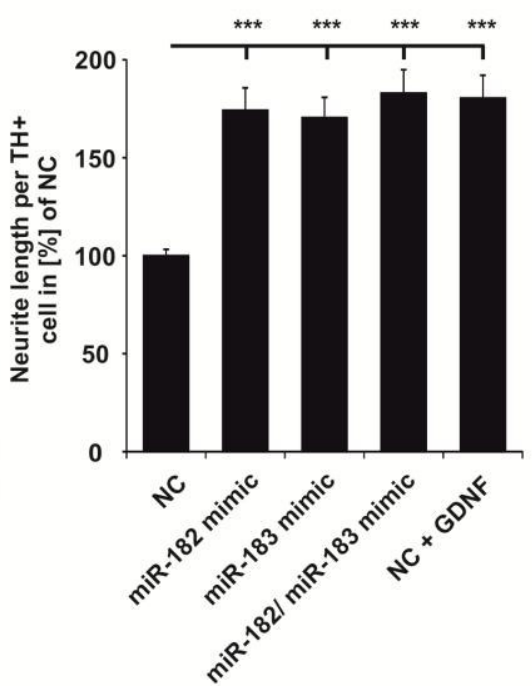

Figure 3.3/1: Increased levels of miR-182 and miR-183 result in longer neurites in dopaminergic neurons in PMN cultures. (a) Experimental layout for transfection experiments. At DIV1 PMN cultures were transfected with a synthetic miRNA mimic or a scrambled NC siRNA (NC). At DIV4 (72 h post-transfection) cultures were fixated for analysis of neurite length. (b) Relative miR-182 (left panel) and miR-183 (right panel) expression in cultures transfected different concentrations of the respective mimic $24 \mathrm{~h}$ post-transfection compared to cultures transfected with NC ( $\mathrm{n}=3$ independent cultures). (c) Representative micrographs of dopaminergic PMN transfected with NC, the respective miRNA mimics or NC and treated with GDNF [10 $\mathrm{ng} / \mathrm{ml}$ ]. PMN were immunostained against TH (scale bar $=50 \mu \mathrm{m}$ ). (d) Quantification of the total neurite length per TH + cell normalized to NC transfected cultures $(n=3$ independent cultures; 2 coverslips per condition; 2 areas of $2000 \mu \mathrm{m}^{2}$ per coverslip; at least $50 \mathrm{TH}+$ neurons per coverslip; mean \pm SEM; one-way ANOVA with Dunnett's post-hoc test).

To analyze the effect of increased miR-182 and miR-183 on dopaminergic PMNs, cultures were transfected at DIV 1 with $5 \mathrm{nM}$ negative control siRNA (NC siRNA) or miRNA 
mimic. For comparison of miRNA effects with GDNF effects, PMNs were transfected with $5 \mathrm{nM}$ NC siRNA and treated with $10 \mathrm{ng} / \mathrm{ml}$ GDNF. At DIV 4 (72 h post-transfection) PMN cultures were fixed and immunostained against TH to label the dopaminergic neurons. Figure 3.3/1 c shows representative micrographs of $\mathrm{TH}$-immunolabelled dopaminergic neurons in the different experimental conditions. The total neurite length per TH-positive neuron relative to NC siRNA transfected neurons was quantified and is displayed in figure 3.3/1 d. After miR-182 mimic transfection dopaminergic PMNs showed a significant increase in neurite length compared to cultures transfected with NC siRNA $(174.3 \pm 11.3$ \%). Also miR-183 mimic transfection led to significantly longer neurites in dopaminergic PMNs (170.4 $\pm 10.3 \%)$. A combined transfection of miR-182 and miR-183 mimics showed just a small additional increase in neurite length of dopaminergic PMNs compared to the singular transfection $(183.9 \pm 12.0 \%)$. Thus, there was no additive effect of both miRNAs visible. Nevertheless, also the effect of the combined transfection on dopaminergic neurite length was significant compared to the effect of NC. PMN cultures transfected with NC siRNA and treated with GDNF also showed a significant increase in neurite length of dopaminergic neurons (180.4 $\pm 11.6 \%)$. Interestingly, the effect mediated by increased miR-182 and miR-183 resembled the effect of GDNF treatment on neurite length of dopaminergic cells.

\subsubsection{Increased miR-182 and miR-183 protect dopaminergic PMNs from MPP ${ }^{+}$ induced neurotoxicity}

In order to analyze a potential neuroprotective effect of increased miR-182 and miR-183 levels in dopaminergic PMNs, the $\mathrm{MPP}^{+}$neurotoxicity model was applied. PMN cultures were transfected with miRNA mimics or NC siRNA at DIV 1 . At DIV 2 (24 h posttransfection) $\mathrm{MPP}^{+}$was supplied to the cell culture medium at a final concentration of 2 $\mu \mathrm{M}$ for $24 \mathrm{~h}$. At DIV 3, cell culture medium was removed completely and fresh medium was added. One day after the medium change (DIV 4) the cells were fixed and dopaminergic cell survival was analyzed after TH immunostaining (figure 3.3/2 a).

In figure $3.3 / 2$ b representative micrographs of all experimental conditions are shown. 


\section{a}

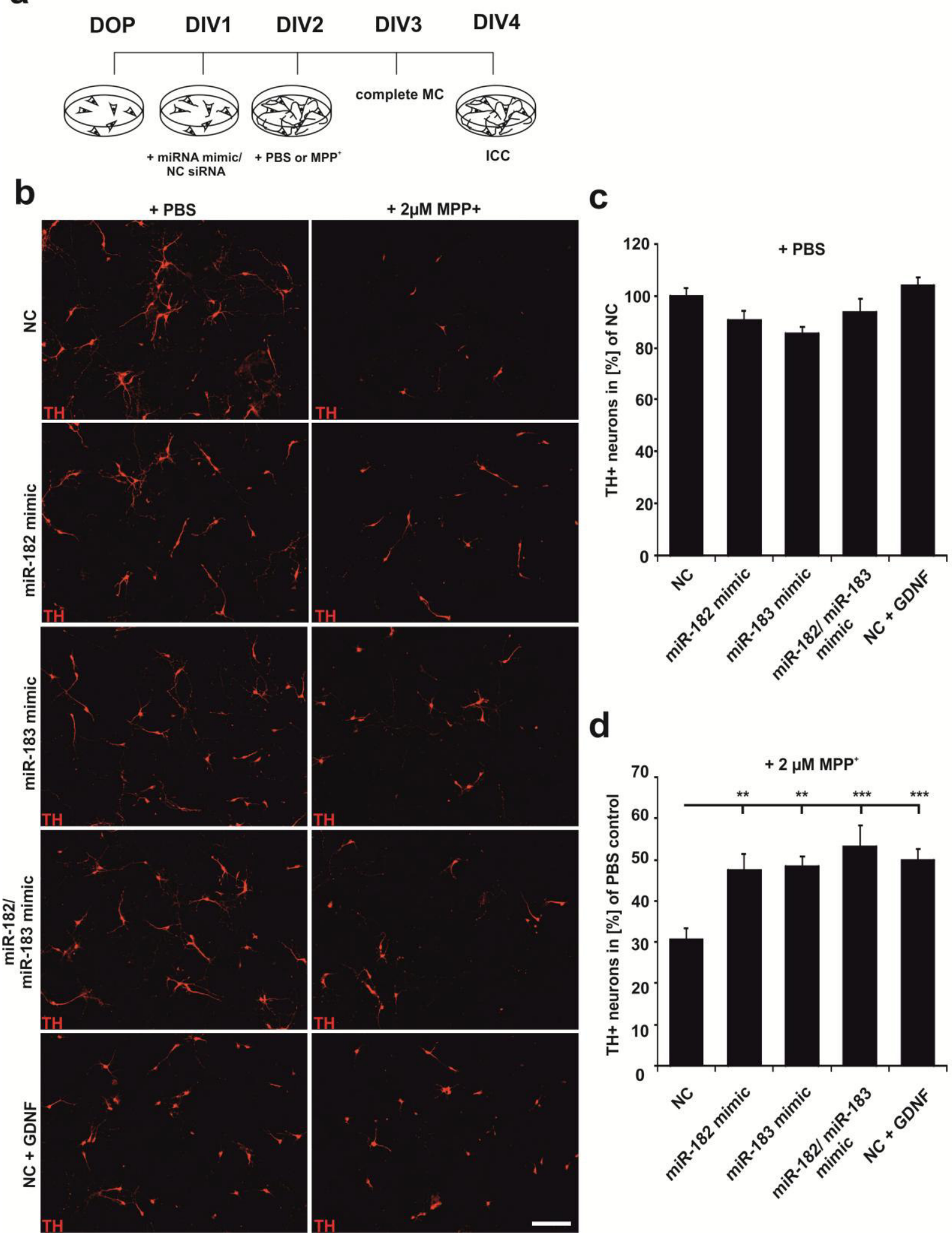

Figure 3.3/2: Increase in miR-182 and miR-183 leads to a higher survival of dopaminergic PMNs after MPP ${ }^{+}$ treatment. (a) Time course of experimental procedure. (b) Representative micrographs of PMN cultures transfected with miRNA mimic, NC or NC + GDNF treatment and treated with PBS (left panel) or $2 \mu \mathrm{M} \mathrm{MPP}{ }^{+}$(right panel) for $24 \mathrm{~h}$. Cultures were immunostained against TH (scale bar $=100 \mu \mathrm{m}$ ). (c) Quantification of TH + PMNs after transfection with miRNA mimics, NC or treatment with $10 \mathrm{ng} / \mathrm{ml}$ GDNF and with addition of PBS for $24 \mathrm{~h}$ as an experimental control. (d) Relative quantification of surviving TH + PMNs after transfection with miRNA mimic, NC or treatment with GDNF and 
addition of $2 \mu \mathrm{M} \mathrm{MPP}+$ for $24 \mathrm{~h}$ normalized to PBS treated cells ( $\mathrm{n}=3$ independent cultures; 2 coverslips per condition; 2 areas of $2000 \mu \mathrm{m}^{2}$ per coverslip). Data is given as mean \pm SEM; one-way ANOVA and Dunnett's test post-hoc test.

The left panel shows micrographs of PMN cultures as controls, transfected with the different oligos and treated with PBS instead of $\mathrm{MPP}^{+}$. The corresponding quantification is displayed in figure $3.3 / 2 \mathrm{c}$. The relative number of $\mathrm{TH}$ positive neurons normalized to the dopaminergic neuron number in PMN cultures transfected with NC siRNA is shown.

There was no significant difference between the number of TH positive PMNs in NC siRNA transfected PMNs and miR-182 (90.6 $\pm 7.0 \%)$ and miR-183 mimic (85.4 $\pm 5.1 \%)$ transfected cultures. The combined transfection with both miRNA mimics had also no significant effect on dopaminergic cell numbers in PBS treated PMNs (93.6 $\pm 7.9 \%)$. The application of GDNF on NC siRNA transfected PMNs had also no significant effect on dopaminergic neuron cell numbers compared to NC siRNA transfected PMNs (104.3 \pm 7.3 $\%)$.

Representative micrographs of PMNs transfected with the different oligos and treated with the neurotoxin $\mathrm{MPP}^{+}$are displayed in the right panel of figure $3.3 / 2 \mathrm{~b}$. The quantification of TH positive neurons after $\mathrm{MPP}^{+}$treatment (figure 3.3/2 d) shows that in PMNs transfected with NC siRNA only $30.5 \pm 2.9 \%$ of the dopaminergic cells survived the $\mathrm{MPP}^{+}$treatment. Cultures transfected with miR-182 mimic showed a significantly higher survival of $47.6 \pm 3.8 \%$ compared to NC siRNA transfected PMNs. A significant neuroprotective effect is also visible in PMNs transfected with miR-183 mimic, where 48.2 $\pm 2.7 \%$ of dopaminergic neurons survived. Again the combined transfection of miR-182 and miR-183 mimic led to no additional effect, but still there was a significantly higher survival of TH positive neurons $(53.3 \pm 5.1 \%)$ compared to NC siRNA treated cells. PMNs transfected with NC siRNA and treated with GDNF showed also a significantly higher number of surviving dopaminergic neurons. With $49.4 \pm 2.9 \%$ survival, the neuroprotective effect of GDNF treatment is again comparable to the effects of increased miR-182 and miR-183 levels on dopaminergic neurons. 


\subsubsection{Increased miR-182 and miR-183 levels improve neurite regeneration in dopaminergic PMN}

For assessment of the neurite regeneration stimulatory potential of miR-182 and miR-183 increase, a mechanical neurite transection of PMN cultures was performed. At DIV 1 cells were transfected with either miRNA mimics or NC siRNA. For comparison of miRNA and GDNF effects another condition was included in which PMNs were transfected with NC siRNA and treated with GDNF. 24 h post-transfection (DIV 2) the PMN neurites were mechanically transected by application of a scratch lesion that was performed using a $200 \mu$ pipette tip. Each coverslip received two scratch lesions that were applied in the same orientation and covered the diameter of the coverslip. Two days after the neurite transection (DIV 4) PMNs were fixed and TH immunolabelling was performed (experimental layout see figure $3.3 / 3$ a). Neurite regeneration was evaluated by measuring the length of dopaminergic neurites crossing the scratch border.

In NC siRNA transfected cultures a limited number of dopaminergic neurites are regenerating across the scratch border as shown in representative pictures in figure $3.3 / 3$ b.

In comparison, miR-182 mimic transfected PMNs showed a strong significant increase in neurite regeneration $(315.8 \pm 51.0 \%)$ as indicated in figure $3.3 / 3 \mathrm{c}$ which shows the total length of TH-positive neurites growing over the scratch border. Also in PMNs transfected with miR-183 mimics a significant increase in dopaminergic neurite regeneration was observed $(221.3 \pm 27.1 \%)$. The combined transfection of both miRNAs led also to significant increase in TH-positive neurites growing over the scratch border (327.2 \pm 34.7 $\%)$, although again no additive effect of both miRNAs was visible. GDNF treatment also increased the regenerative growth of dopaminergic neurites $(255.1 \pm 34.6 \%)$ in PMNs after scratch lesion, which in this case is not as high as in cultures transfected with miR182 mimics. The quantification shown in figure $3.3 / 3 \mathrm{c}$ is not taking into account if the regenerating neurites are longer or if there are just more regenerating dopaminergic neurites. In order to exclude the bias of neurite number, a second quantification was performed. This time, only the length of the 10 longest neurites crossing the scratch border was evaluated and the mean length was calculated. The results are displayed in figure $3.3 / 3 \mathrm{~d}$. 


\section{a}

$\begin{array}{llll}\text { DOP } & \text { DIV1 DIV2 } & \text { DIV4 }\end{array}$

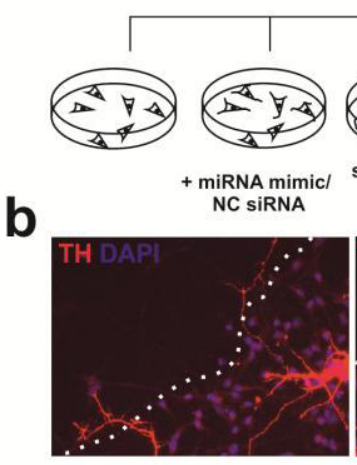

NC
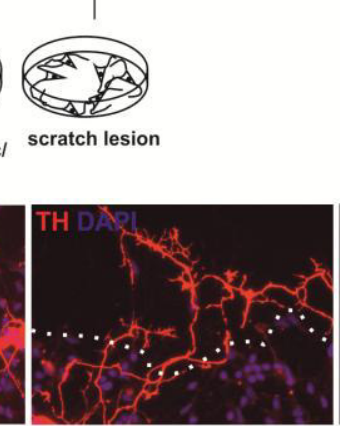

miR-182 mimic

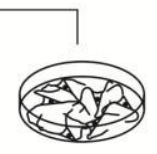

ICC

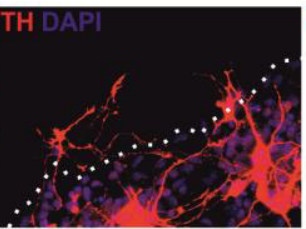

miR-183 mimic

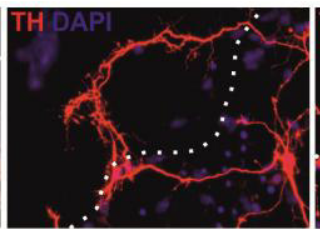

miR-182/ miR-183 mimic

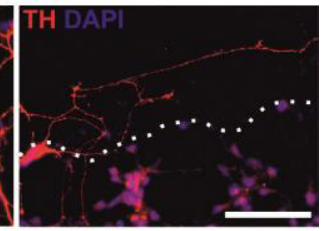

$N C+G D N F$

C
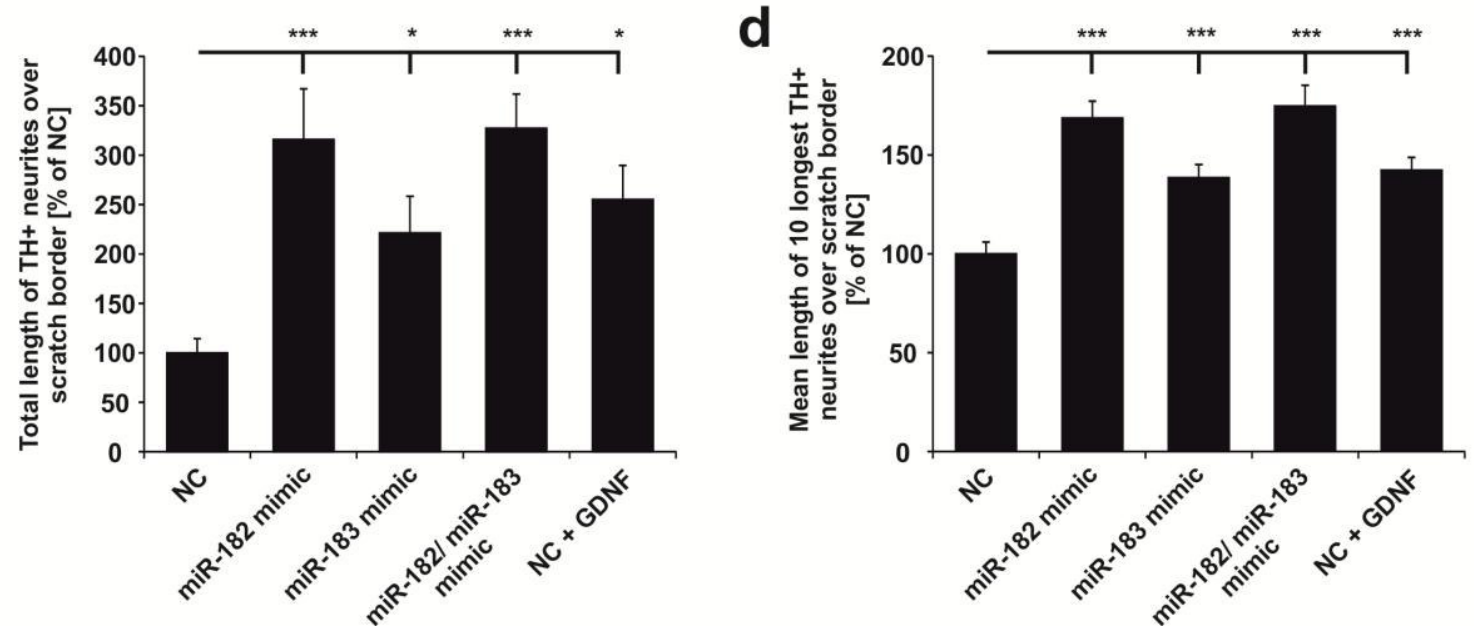

Figure 3.3/3: miR-182 and miR-183 transfection results in increased regeneration of $\mathrm{TH}+$ neurites after mechanical scratch lesion. (a) Experimental layout. (b) Representative micrographs of TH immunostained and DAPI counter stained miRNA mimic, NC or GDNF treated PMN cultures after mechanical scratch lesion. The scratch border is indicated by the white dotted line $($ scale bar $=50 \mu \mathrm{m}$ ). (c) Quantification of the total length of $\mathrm{TH}+$ neurites over the scratch border relative to NC transfected PMNs ( $\mathrm{n}=3$ independent cultures; 2 coverslips per condition; 2 scratch lesions per coverslip). (d) Quantification showing the mean length of the 10 longest $\mathrm{TH}+$ neurites crossing the scratch border relative to NC transfected PMN cultures ( $n=3$ independent cultures; 2 coverslips per condition; 2 scratch lesions per coverslip). Data is given as mean $\pm \mathrm{SEM}$; one-way ANOVA and Dunnett's test post-hoc test.

Compared to NC siRNA, miR-182 and miR-183 mimic transfection significantly increased the length of regenerating dopaminergic neurites (miR-182: $168.6 \pm 8.5 \%$; miR183: $138.5 \pm 6.7 \%)$. The combined transfection of both miRNAs led to no further increase $(174.6 \pm 10.6 \%)$ in regenerating neurite length compared to the singular transfection of miR-182. The effect of GDNF treatment was not as strong (142.3 $\pm 6.4 \%)$ as miR-182 transfection but was nevertheless significant compared to NC siRNA-transfected PMNs. 


\subsection{Neurite outgrowth, neuroprotection and neurite regeneration - Mechanisms underlying the beneficial effects of miR-182 and miR-183 increase in dopaminergic PMNs}

Taken together the present results suggest that GDNF-regulated miR-182 and miR183 play an important role in neurite outgrowth and regeneration of dopaminergic neurons in vitro. Furthermore, the results of the $\mathrm{MPP}^{+}$-intoxication experiment demonstrate that those miRNAs also exert dopaminergic neuroprotection. The upcoming chapter aims to elucidate the mechanisms behind the observed effects. In order to show that the observed effects are not caused by mere transfection of miRNA mimics per se, but are indeed specific for miR-182 and miR-183 increase, PMNs were transfected with miR-1a-3p. miR-1a-3p is a heart-specific miRNA that was already used as a control and showed no effect on neurite outgrowth in cortical neurons (Vo et al. 2006). For further investigation of the signaling pathways involved in miR-182 and miR-183 mediated effects, protein levels of their already experimentally validated target genes as well as of key mediators of survival and growth associated signaling pathways were analyzed.

\subsubsection{Increased neurite growth, regeneration and neuronal survival are not an effect of miRNA increase per se}

To exclude the possibility that the observed effects of miR-182 and miR-183 increase on neuronal survival and neurite growth are not an effect of miRNA overexpression per se, all experiments were repeated according to the previously described protocols transfecting miR-1a-3p.

In figure 3.4/1 a the increase in miR-1a-3p detection by qRT-PCR $24 \mathrm{~h}$ after transfection with $5 \mathrm{nM}$ miRNA mimic is displayed. In order to analyze the effect of miR$1 a-3 p$ on neurite outgrowth of dopaminergic neurons transfected PMN cultures were fixed at DIV 4 and immunolabeled for TH. There was no significant difference in neurite length of TH positive dopaminergic neurons between cells transfected with miR-1a-3p mimic (100.7 $\pm 4.7 \%)$ and cells transfected with a NC siRNA as shown in figure 3.4/1 b. Furthermore the effect of miR-1a-3p on dopaminergic neuronal survival after MPP ${ }^{+}$ treatment was evaluated. 
a

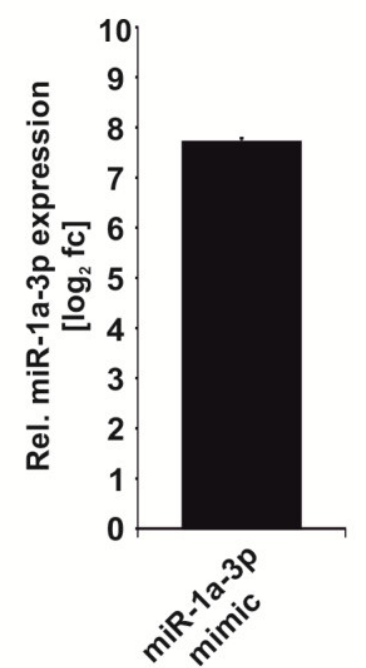

C

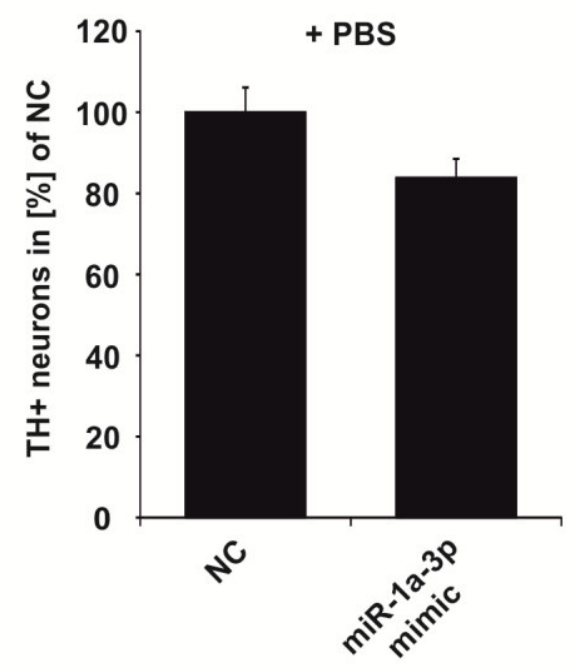

e

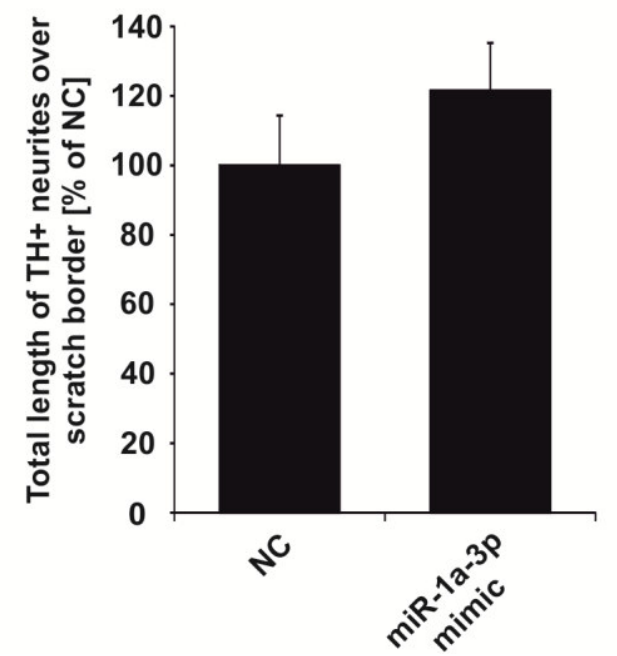

b
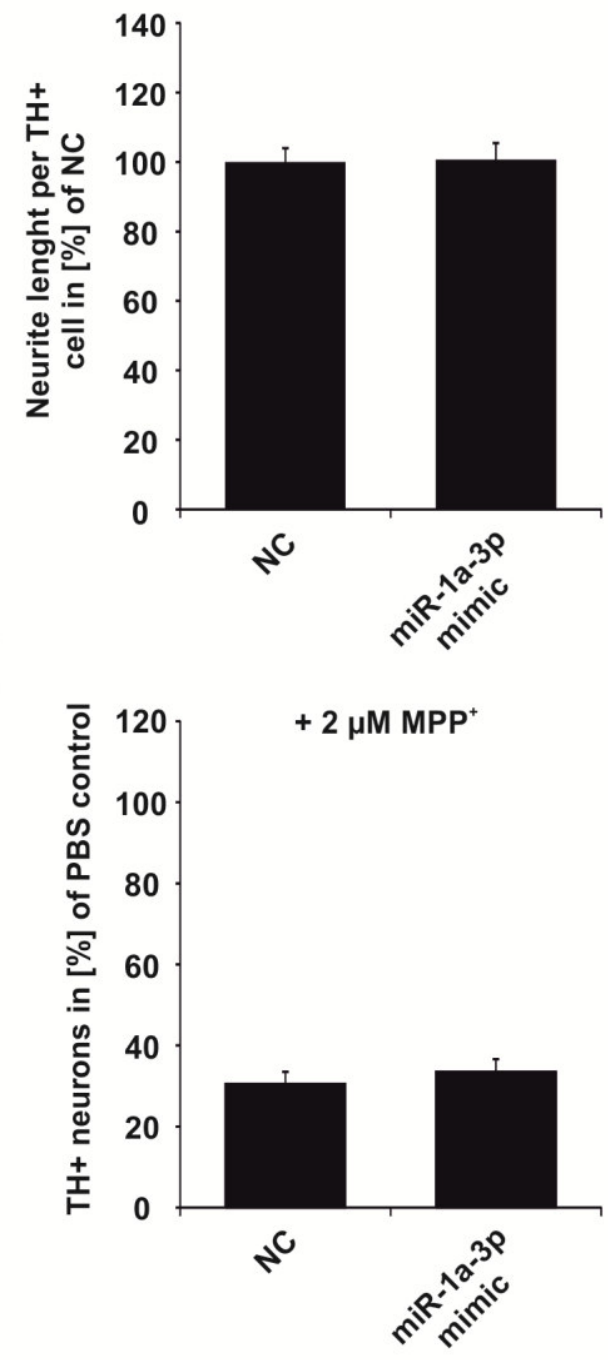

f

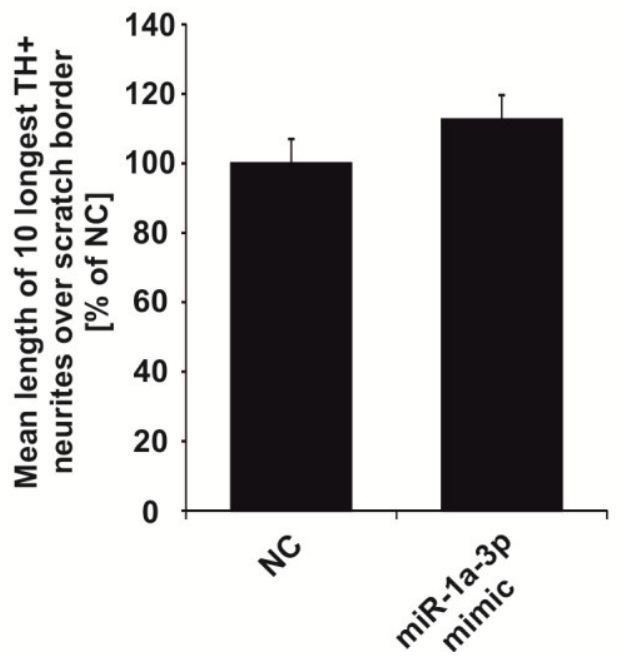

Figure 3.4/1: Effects on neuronal survival and neurite growth and regeneration are not caused by miRNA overexpression per se. (a) Relative miR-1a-3p expression in cultures transfected with 5 nM miRNA mimic normalized to expression levels in cultures transfected with NC ( $n=3$ independent cultures). (b) Quantification of neurite length of dopaminergic neurons in PMN cultures transfected with miR-1a-3p and NC ( $\mathrm{n}=3$ independent cultures; 2 coverslips per condition; 2 areas of $2000 \mu^{2}$ per coverslip; at least $50 \mathrm{TH}+$ neurons per coverslip). (c) Quantification of TH + PMNs 
after transfection with miR-1a-3p mimic or NC and with addition of PBS for $24 \mathrm{~h}$ as an experimental control. (d) Relative quantification of surviving TH + PMNs after transfection with miR-1a-3p or NC and addition of $2 \mu \mathrm{M}$ MPP+ for $24 \mathrm{~h}$ normalized to PBS treated cells ( $n=3$ independent cultures; 2 coverslips per condition; 2 areas of $2000 \mu m^{2}$ per coverslip). (e) Quantification of the total length of TH + neurites over the scratch border relative to NC transfected PMNs ( $\mathrm{n}=3$ independent cultures; 2 coverslips per condition; 2 scratch lesions per coverslip). (f) Quantification showing the mean length of the 10 longest TH + neurites crossing the scratch border relative to NC transfected PMN cultures ( $n$ = 3 independent cultures; 2 coverslips per condition; 2 scratch lesions per coverslip). Data is given as mean \pm SEM; oneway ANOVA and Dunnett's test post-hoc test.

One day after transfection, cultures were treated with $2 \mu \mathrm{M} \mathrm{MPP}{ }^{+}$for $24 \mathrm{~h}$. At DIV 4 cells were fixed and immunolabeled for TH to analyze dopaminergic cell numbers. Figure 3.10 c shows the effect of miR-1a-3p on dopaminergic cell numbers without additional treatment. There is no significant difference between NC siRNA-treated cultures and miR1a-3p mimic-transfected PMNs in TH-positive cell numbers. After $\mathrm{MPP}^{+}$treatment dopaminergic cell numbers in NC siRNA-transfected cells decreased to $30.5 \pm 2.9 \%$ and in miR-1a-3p mimic-transfected cultures to $33.5 \pm 3.0 \%$ (figure $3.4 / 1 \mathrm{~d}$ ), there is no significant difference in survival of dopaminergic neurons after $\mathrm{MPP}^{+}$treatment in miR1a-3p transfected PMNs compared to NC siRNA transfected PMNs.

Regarding neurite regeneration of dopaminergic neurons two days after mechanical scratch lesion, there was no significant difference between NC siRNA- and miR-1a-3p mimic-transfected cultures (figure 3.4/1 e and f). The total length of regenerating dopaminergic neurons over the scratch border in miR-1a-3p mimic transfected cells was $121.5 \pm 13.7 \%$ compared to NC siRNA transfected PMNs. The mean length of the 10 longest dopaminergic neurites over the scratch border in miR-1a-3p transfected cells was $112.6 \pm 7.0 \%$ of the NC.

Taken together, the miR-1a-3p transfection experiments indicate that the observed effects of miR-182 and miR-183 mimic transfection on dopaminerigc neurons are specific effects and not simply caused by miRNA mimic transfection per se. In the following chapter the possible mechanisms underlying the pro-dopaminergic effects of miR-182 and miR-183 will be further investigated. 


\subsubsection{Increased miR-182 and miR-183 levels lead to a reduction in Foxo3 and Foxo1 protein expression}

For further investigation of the mechanisms underlying miR-182 and miR-183 increase-mediated beneficial effects on dopaminergic neurons, the protein expression levels of already experimentally validated target genes for miR-182 and miR-183 were analyzed. It was previously reported that miR-182 regulates the protein expression levels of the transcription factors Foxo1 and Foxo3 (Guttilla \& White 2009; Segura et al. 2009). Taok1 was previously described as regulated by miR-183 (Patel et al. 2013), but also Foxo1 was predicted to be a target of miR-183 (TargetScan Mouse 6.2). All of these target proteins were previously reported to have an impact on neuronal survival and / or development (Chong et al. 2008; Tavares et al. 2013; Yuan et al. 2009). Protein levels were analyzed by Western blot in lysates prepared from PMN cultures transfected at DIV1 with miR-182, miR-183 or NC siRNA. Lysates were prepared $24 \mathrm{~h}$ post-transfection.

Western blot analyses of Foxo3 protein levels revealed a significant downregulation of Foxo3 in miR-182 transfected cultures $24 \mathrm{~h}$ post-transfection (Figure 3.4/2 a). Foxo3 levels were decreased to $75.9 \pm 5.6 \%$ in cells transfected with miR-182. Although not significant, a similar trend could be observed in cultures treated with GDNF $(81.7 \pm 5.8 \%)$. Transfection of miR-183 mimic had no effect on the protein levels of Foxo3 in PMN cultures $(88.6 \pm 7.8 \%)$. Regarding the protein levels of Foxo1 after transfection with miRNA mimics or treatment with GDNF (figure $3.4 / 2$ b), quantification of 5 independent cultures showed a significant decrease in Foxo1 levels in all three treatment groups (miR-182: $63.4 \pm 2.4 \%$; miR-183: $68.0 \pm 6.4 \%$; NC + GDNF: $53.4 \pm 5.7 \%$ ). Analyses of 5 independent cultures revealed no significant effect of miR-182 or miR-183 transfection, as well as GDNF treatment on Taok1 protein expression in PMNs, although there was a trend to decreased Taok1 levels in cells transfected with miR-183 (66.8 5.6 $\%$ ) and in PMNs treated with GDNF (72.4 $\pm 11.3 \%$ ) (figure 3.4/2 c). 
a
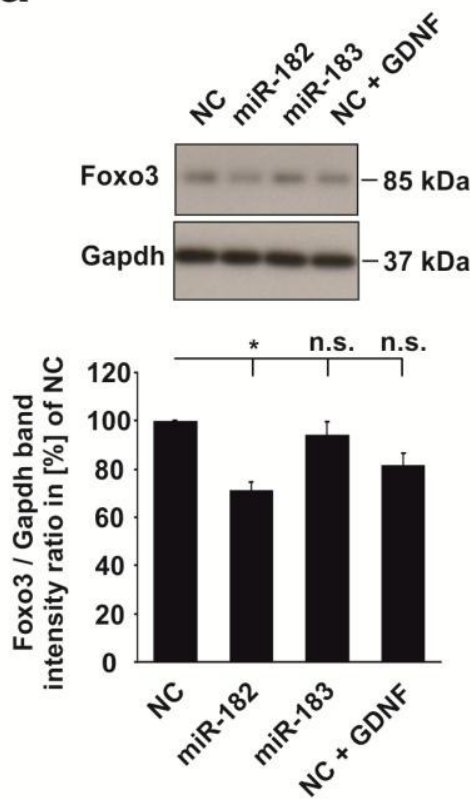

b

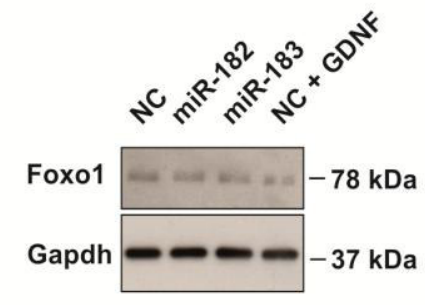

C

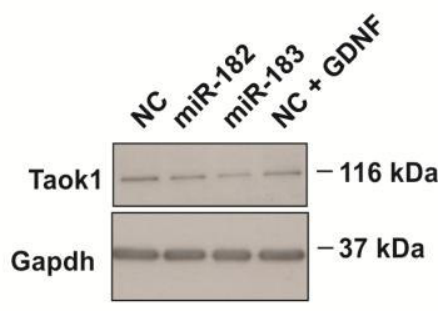

Figure 3.4/2: Increased levels of miR-182 and miR-183 lead to a decrease in Foxo protein levels. Western blot analyses of Foxo3 (a), Foxo1 (b) and Taok1 (c) protein levels in miR-182, miR-183 or GDNF treated PMNs 24 h post-transfection. Band intensities for Foxo3 show a reduction in miR-182 transfected cells that is also confirmed by the quantification below (a) ( $n=5$ independent cultures). Foxo1 protein levels (b) are decreased in PMNs transfected with miR-182, miR183 or treated with GDNF ( $\mathrm{n}=4$ independent cultures). There is a trend for decreased Taok1 levels in PMNs transfected with miR-183, although this is not significant (c) ( $n=4$ independent cultures). Data is given as mean \pm SEM; one-way ANOVA and Dunnett's test post-hoc test.

\subsubsection{Effects of increased miR-182 and miR-183 on survival and growth associated signaling pathways}

For further elucidation of the mechanisms underlying miR-182 and miR-183 mediated beneficial effects on dopaminergic neurons, the levels of proteins involved in survival and growth associated signaling pathways were evaluated in miR-182 and miR183 transfected PMNs. Lysates were prepared $24 \mathrm{~h}$ post-transfection and Western blot analyses were performed for the known survival / growth associated proteins Akt, pAkt, Erk, pErk, Bcl2, Stat3 and phospho-S6. 


\subsubsection{1 miR-182 and miR-183 mimic transfection lead to increased phosphorylation of} Akt

Akt is a protein involved in the PI3K-Akt signaling pathway, a signal transduction pathway that mediates cellular survival and growth in response to external stimuli and plays an important role also in neuronal cells (Brunet et al. 2001; Kennedy et al. 1997). Here, the levels of Akt and its phosphorylated active form pAkt were analyzed in miR-182 and miR-183 transfected PMNs 24 h post-transfection.

Transfection of miR-182 and miR-183, as well as GDNF treatment had no effect on Akt protein levels as displayed in figure 3.4/3 a (miR-182: $102.8 \pm 2.5 \%$; miR-183: $91.6 \pm$ $3.2 \%$; NC + GDNF: $96.3 \pm 2.3 \%$ ). Nevertheless, miR-182 and miR-183 mimic transfected PMNs showed a significant increase in pAkt levels and therefore a higher activation of the PI3K-Akt signaling pathway (figure $3.4 / 3$ b).

a

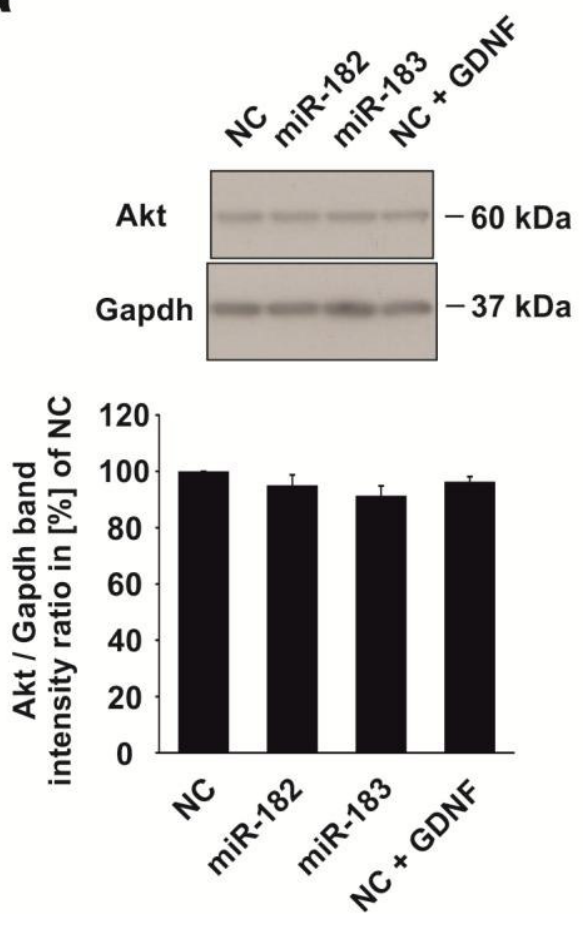

b

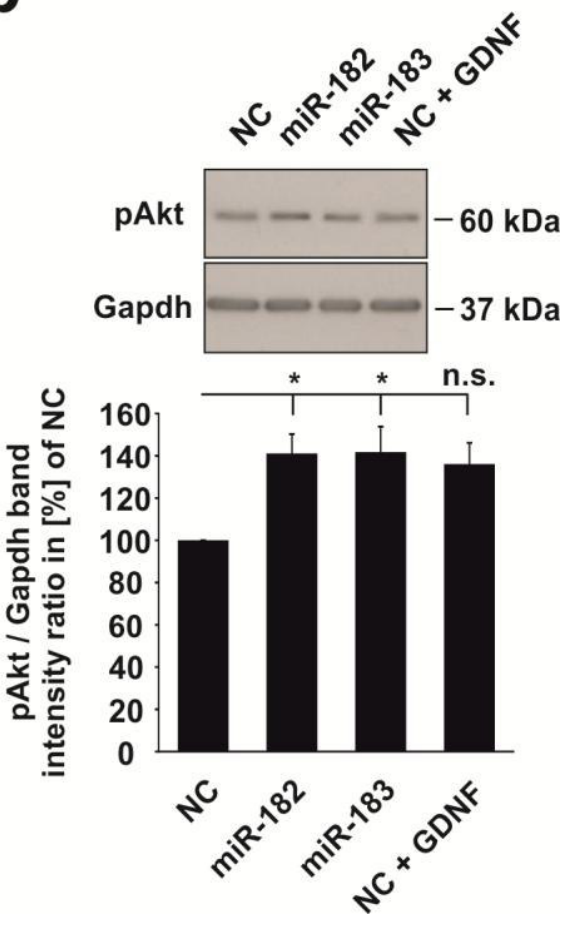

Figure 3.4/3: Increased miR-182 and miR-183 lead to increased phosphorylation of Akt. Western blot analyses of Akt (a) and phospho-Akt (b) (pAkt) protein levels in miR-182, miR-183 or GDNF treated PMNs $24 \mathrm{~h}$ post-transfection. Band intensities for Akt show no effect of miRNA increase or GDNF treatment that is also displayed in the quantification below (a) ( $n=5$ independent cultures). In miR-182 and miR-183 transfected PMNs there is a significant increase in pAkt detectable (b) ( $n=5$ independent cultures). Data is given as mean \pm SEM; one-way ANOVA and Dunnett's test post-hoc test. 
In miR-182 mimic-transfected cells the pAkt levels reached on average $141.1 \pm 10.1 \%$ of controls. Transfection of miR-183 led to an increase to $141.8 \pm 13.2 \%$ in pAkt levels. Transfection with the NC siRNA followed by GDNF treatment resulted in a non-significant trend to increased Akt phosphorylation (136.0 $\pm 12.3 \%)$.

\subsubsection{Transfection of miR-182 and miR-183 lead to a trend of increased MAPK (Erk) signaling}

The MAPK signaling pathway plays a critical role in mediation of cell survival and growth and is also important for neuronal plasticity (Bonni et al. 1999; Impey et al. 1999). The effect of miR-182 and miR-183 increase on the MAPK signaling pathway in PMNs was investigated by evaluation of Erk1 and Erk2 (Mapk) levels, as well as the evaluation of the levels of their activated phosphorylated forms pErk1 and pErk2.

Increased miR-182 levels in PMNs resulted in a trend for increased Erk1 and Erk2 expression as shown in figure 3.4/4 a, which was not significant (Erk1: $134.1 \pm 10.0 \%$; Erk2: $121.6 \pm 8.2 \%)$. Furthermore, miR-182 transfection led to an even stronger, yet insignificant trend of increased pErk1 (292.0 $\pm 88.2 \%)$ and pErk2 (180.2 $\pm 23.6 \%)$ in PMNs (figure 3.4/4 b). miR-183 transfection led also to a small increase in Erk1 (126.3 $4.7 \%$ ) and Erk2 (118.2 $\pm 5.9 \%$ ) levels, as well as to a higher phosphorylation of Erk1/2 (pErk1: $205.1 \pm 38.7 \%$; pErk2: $141.7 \pm 20.9 \%$ ). Transfection with NC siRNA and subsequent treatment with GDNF resulted in comparable increased Erk1/2 and even higher increase in pErk1/2 levels (Erk1: $115.2 \pm 12.8 \%$; Erk2: $117.6 \pm 10.3 \%$; pErk1: $404.5 \pm 42.2 \%$; pErk2: $185.1 \pm 52.6 \%)$. 
a
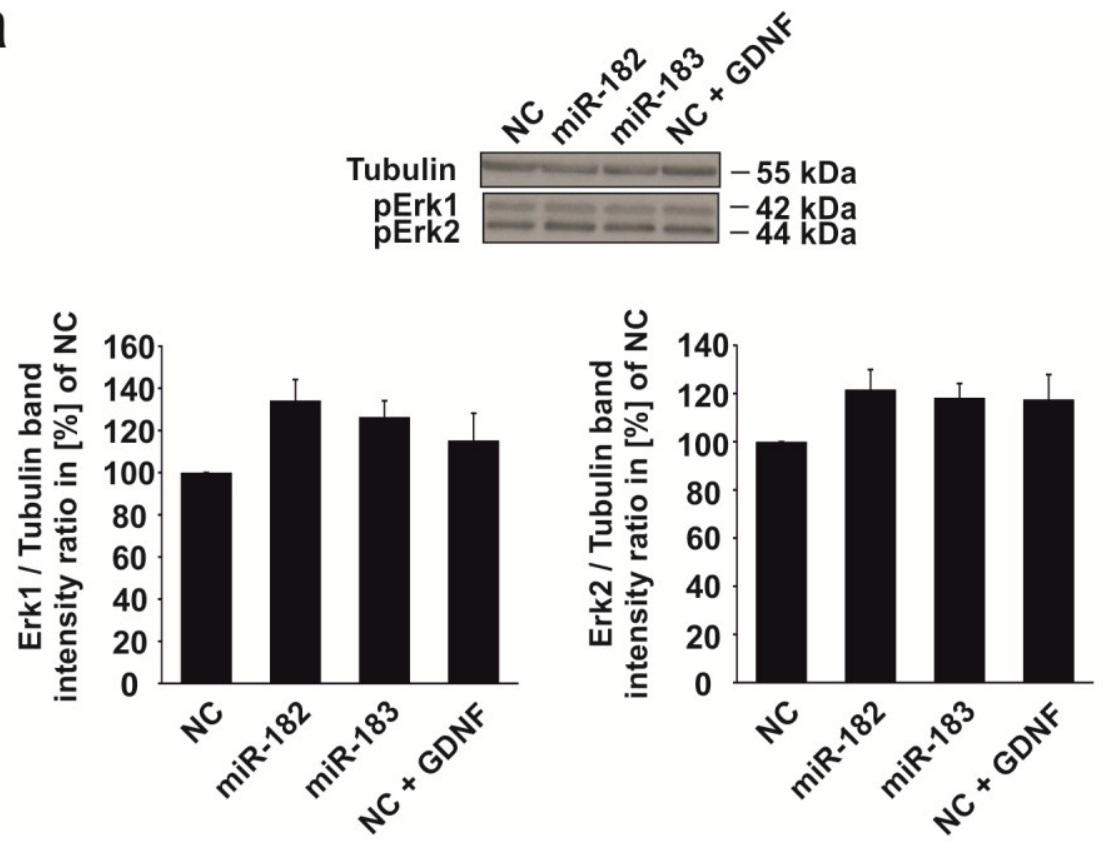

b
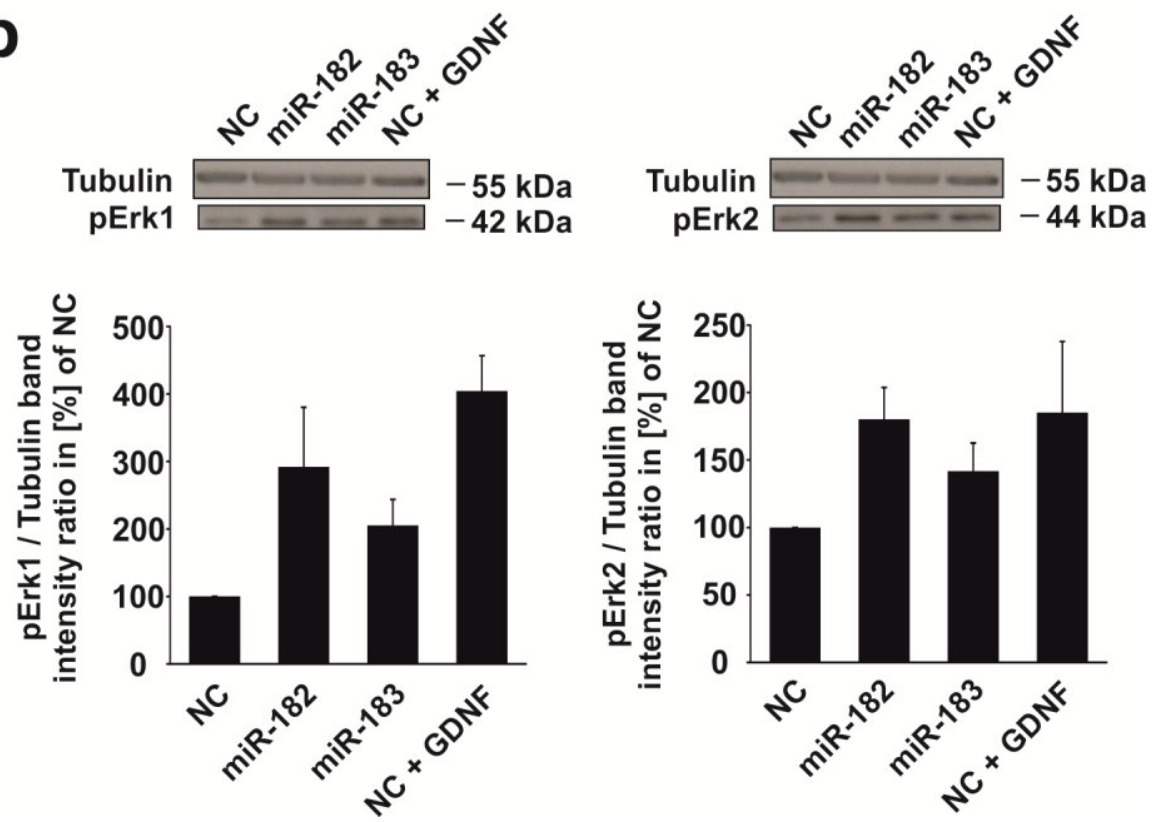

Figure 3.4/4: Higher miR-182 and miR-183 levels lead to trend of increased Mapk (Erk) signaling. Western blot analyses of Erk (a) and phospho-Erk (b) (pErk) protein levels in miR-182, miR-183 or GDNF treated PMNs $24 \mathrm{~h}$ posttransfection. Band intensities for Erk show a trend for higher Erk1 and Erk2 levels in PMNs treated with miRNA mimics or GDNF (a) ( $n=5$ independent cultures). Also in all three treatment groups there is a trend for an increased phosphorylation of Erk1 (pErk1) and Erk2 (pErk2) detectable (b) ( $n=5$ independent cultures). Data is given as mean \pm SEM; one-way ANOVA and Dunnett's test post-hoc test. 


\subsubsection{Influence of increased miR-182 and miR-183 on Stat3, phospho-S6 and Bcl2 expression in PMNs}

In addition to the miR-182 and miR-183 mediated influence on the Akt and MAPK signaling pathways, the effect of those miRNAs on other growth associated proteins was investigated. Protein expression of Stat3, phospho-S6 and Bcl2 in miR-182 and miR-183 mimic transfected PMNs was analyzed $24 \mathrm{~h}$ post-transfection. Stat3 is a transcription factor that is important for cell growth and apoptosis (Zhong et al. 1994). Phospho-S6 is the activated form of the ribosomal component S6 and a downstream target of the mammalian target of rapamycin (mTOR) and is therefore involved in protein translation and associated with cell growth and survival (Jeon et al. 2008). The anti-apoptotic protein $\mathrm{Bcl} 2$ is associated with the mitochondrial membrane and protects cells from oxidative stress induced cell death by prevention of cytochrome c release or binding of the apoptosis-activating factor (Czabotar et al. 2013). Lysates of transfected PMNs were prepared $24 \mathrm{~h}$ post-transfection and Western blotting was performed.

a
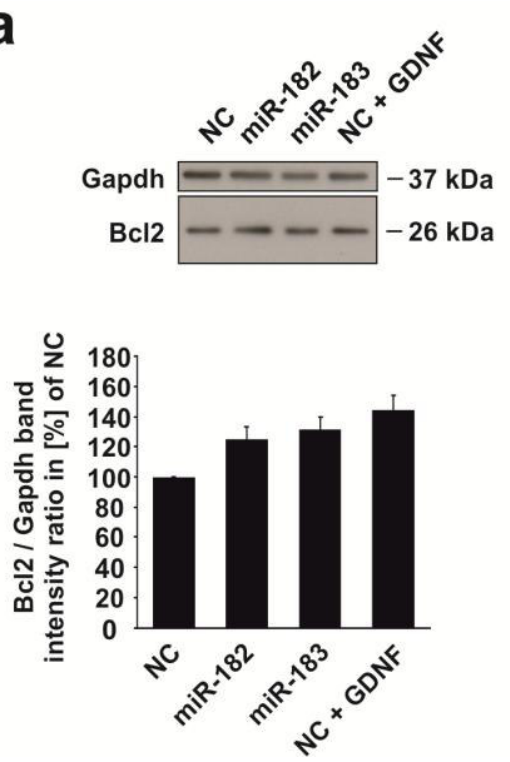

b

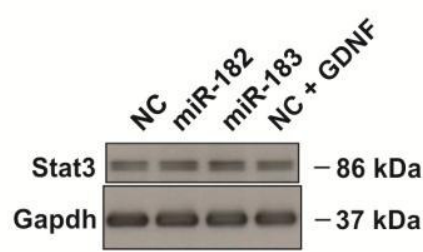

C
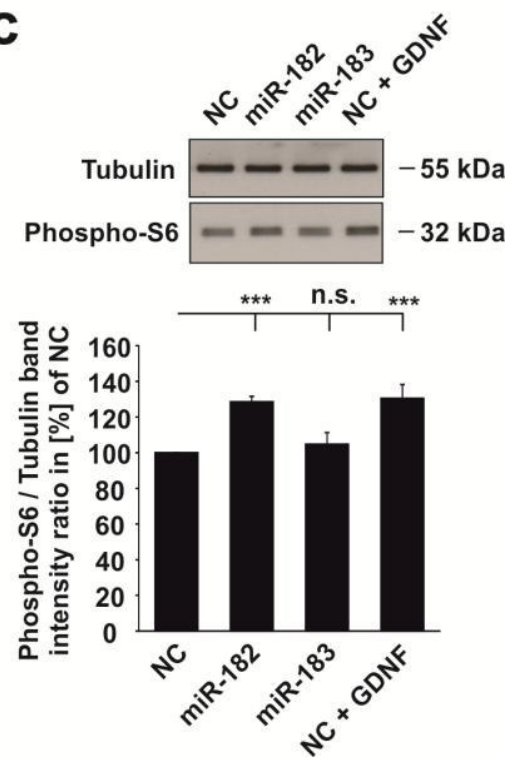

Figure 3.4/5: Effect of miR-182 and miR-183 increase on Bcl2, Stat3 and phospho-S6 protein levels. Western blot analyses of $\mathrm{Bcl} 2$ (a), Stat3 (b) and phospho-S6 (c) protein levels in miR-182, miR-183 or GDNF treated PMNs 24 h posttransfection. Band intensities for Bcl2 show a trend for higher Bcl2 levels in PMNs treated with miRNA mimics or GDNF (a) ( $n=5$ independent cultures). Also in all three treatment groups there is a trend for an increased Stat3 detectable (b) ( $n=5$ independent cultures). Increased miR-182 levels lead to a significant increase in phospho-S6 expression (c) ( $\mathrm{n}=5$ independent cultures). Data is given as mean \pm SEM; one-way ANOVA and Dunnett's test post-hoc test. 
Bcl2 expression increased in miR-182 (131.9 $\pm 9.1 \%)$ and miR-183 (134.9 $\pm 12.2 \%)$ transfected PMNs, as well as after GDNF treatment of the cells $(157.8 \pm 26.7 \%)$, although none of these changes were significant (displayed in figure 3.4/5 a). A similar alteration was detectable in Stat3 protein levels after transfection with miR-182 or miR-183 and treatment with GDNF. miR-182 increase resulted in increased Stat3 levels of $139.7 \pm 14.2$ $\%$, for miR-183 transfection the Stat3 levels were at $130.7 \pm 16.6 \%$ and GDNF treatment increased Stat3 expression to $121.8 \pm 10.7 \%$. These changes did not reach significance (figure 3.4/5 b). Phospho-S6 levels were significantly altered in two of three experimental groups. Transfection of miR-182 mimic increased phospho-S6 levels to $128.6 \pm 3.3 \%$, whereas miR-183 transfection led to no significant changes in phospho-S6 expression (104.8 $\pm 7.2 \%)$. GDNF treatment of NC siRNA transfected PMNs resulted in significantly increased phospho-S6 levels of $130.4 \pm 8.7 \%$ (figure $3.4 / 5$ c). 


\subsection{Effects of increased miR-182 and miR-183 levels in the acute MPTP mouse model for PD}

After demonstrating the beneficial effects of GDNF-regulated miR-182 and miR183 on dopaminergic neurons in culture, the present analysis aimed to elucidate if similar pro-dopaminergic effects could be observed in the adult murine brain in vivo. Therefore, the acute MPTP mouse model for PD was applied. Animals received a stereotactical injection of miR-182 or miR-183 mimics or NC siRNA into the right SNpc and were treated with MPTP for 5 consecutive days beginning at day two post-injection.

The first parts of this chapter are dealing with the optimization of the nigral miRNA mimic injection, whereas the following chapters cover the effects of miR-182 and miR-183 increase in the MPTP mouse model for PD.

\subsubsection{Stereotactical injection of fluorescently labeled NC siRNA leads to transfection of dopaminergic neurons in the murine SNpc}

In order to verify the transfection of dopaminergic neurons in the SNpc by stereotactic injection of miRNA mimics / NC siRNA, a NC siRNA fluorescently labeled with AF-546 in two different concentrations (100 pmol and 500 pmol) with or without lipidbased HiPerfect transfection (HiP) reagent was injected into the right SNpc of mice. 48 hours post-injection animals were perfused and brain tissue was processed for cryosectioning and immunohistochemistry. Sections were immunolabeled for TH to show the transfection of dopaminergic neurons in the SN (figure 3.5/1 a). In figure 3.5/1 b representative micrographs of the ipsilateral and contralateral SNpc of injected animals are shown. Compared to animals injected only with PBS and HiP, the animals injected with fluorescently labeled NC siRNA (AF-546) showed a clear AF-546 signal in SNpc cells. 
a

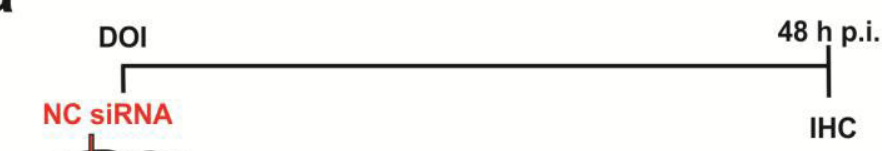

b

\section{uninjected}

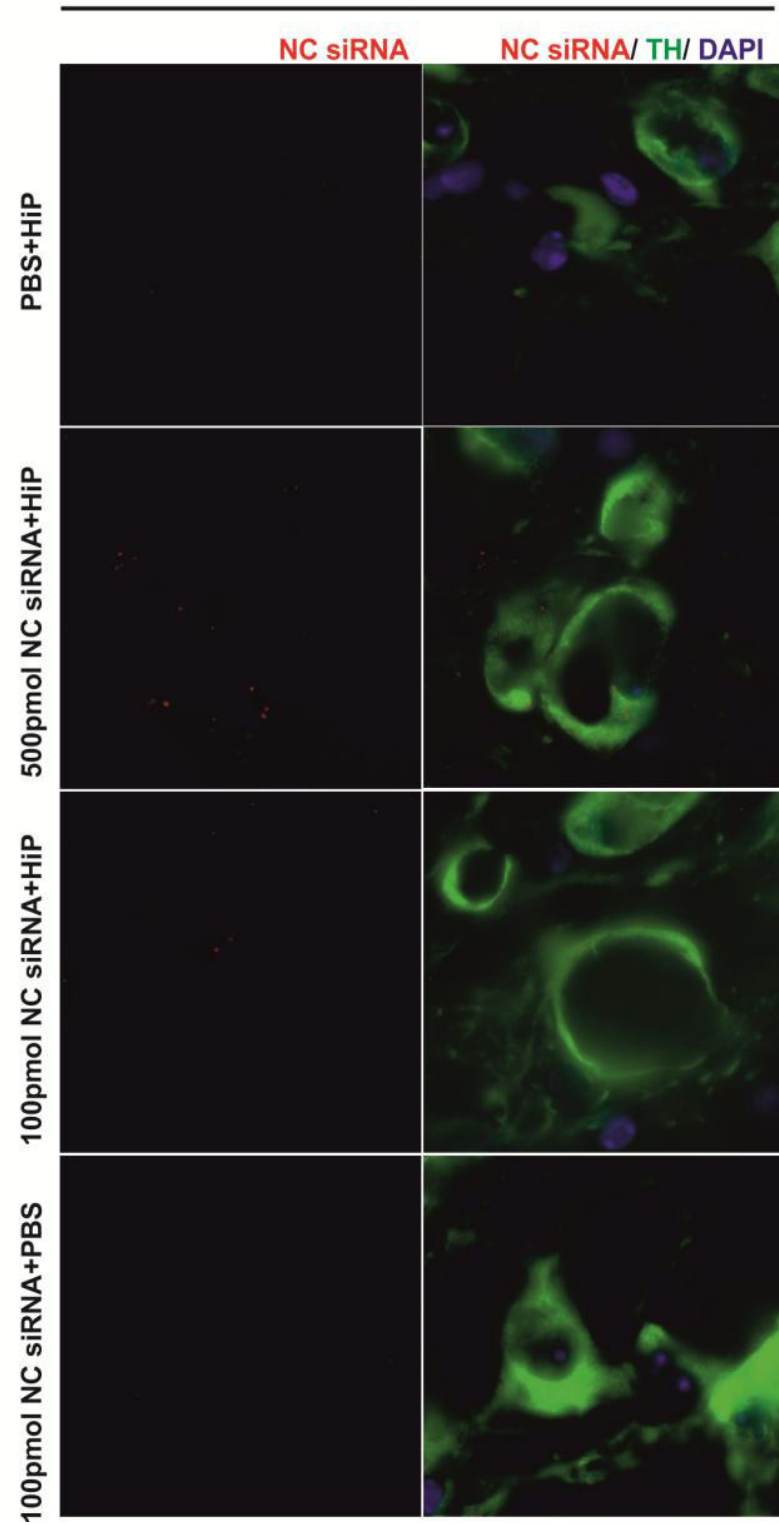

injected

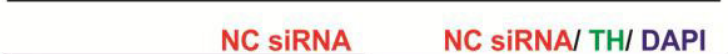

Figure 3.5/1: Transfection of SN dopaminergic neurons of mice with fluorescently labeled NC siRNA in vivo. (a) Experimental layout. Fluorescently labeled NC siRNA at different concentrations and either with or without addition of the lipid-based transfection reagent HiPerfect (HiP) was injected into the right SN of 8 weeks old male C57BI/6J mice (DOI = day of injection). 48 post-injection animals were sacrificed and tissues were prepared for IHC. (b) Representative micrographs of SN in injected animals. Left panels: Contralateral uninjected side showing in the left panel the channel for NC siRNA and in the right panel the merge of NC siRNA, TH and DAPI signals. Right panels: Ipsilateral injected side showing a colocalization of the NC siRNA signal and TH + neuronal cells (scale bar $=20 \mu \mathrm{m}$ ). 
Independent of the transfected amount of siRNA, there was a clear co-localization of the AF-546 signal of the NC siRNA and the TH signal that was used as a marker for dopaminergic neurons, leading to the conclusion that the nigral injection of small RNA species resulted in a transfection of ipsilateral dopaminergic SNpc neurons $48 \mathrm{~h}$ postinjection. At this time point no spreading of the AF-546 labeled siRNA to the contralateral side was detectable. Animals that were injected with a mixture of NC siRNA and HiP seem to show higher transfection efficiencies compared to animals injected just with the siRNA in PBS. Based on these results further experiments were conducted using $250 \mathrm{pmol}$ miRNA mimic or NC siRNA.

\subsubsection{Lipid-based transfection reagent does not lead to increased microglial invasion in the murine SNpc}

With the intention of excluding potential side effects of the used transfection reagent $(\mathrm{HiP})$ on microglial invasion the microglia numbers were analyzed in animals injected with NC siRNA without HiP and in animals injected with NC siRNA with the use of HiP. To this, animals were sacrificed $48 \mathrm{~h}$ post-injection, SNpc sections were immunolabeled for the microglial marker lba 1 and stereological counting of microglial numbers around the injection site was performed (figure 3.5/2 a and b).

In figure $3.5 / 2 \mathrm{c}$ the results of the stereological analyses are displayed. Animals injected with NC siRNA and HiP showed no significant increase in microglial numbers around the injection site compared to animals injected with NC siRNA in PBS only. Consequently, it was concluded that the lipid-based transfection reagent HiP does not lead to an increased microglial invasion around the injection site. On the basis of this result it was decided that injections of small RNA species in further experiments were performed with addition of HiP to the oligo mixture. 
a
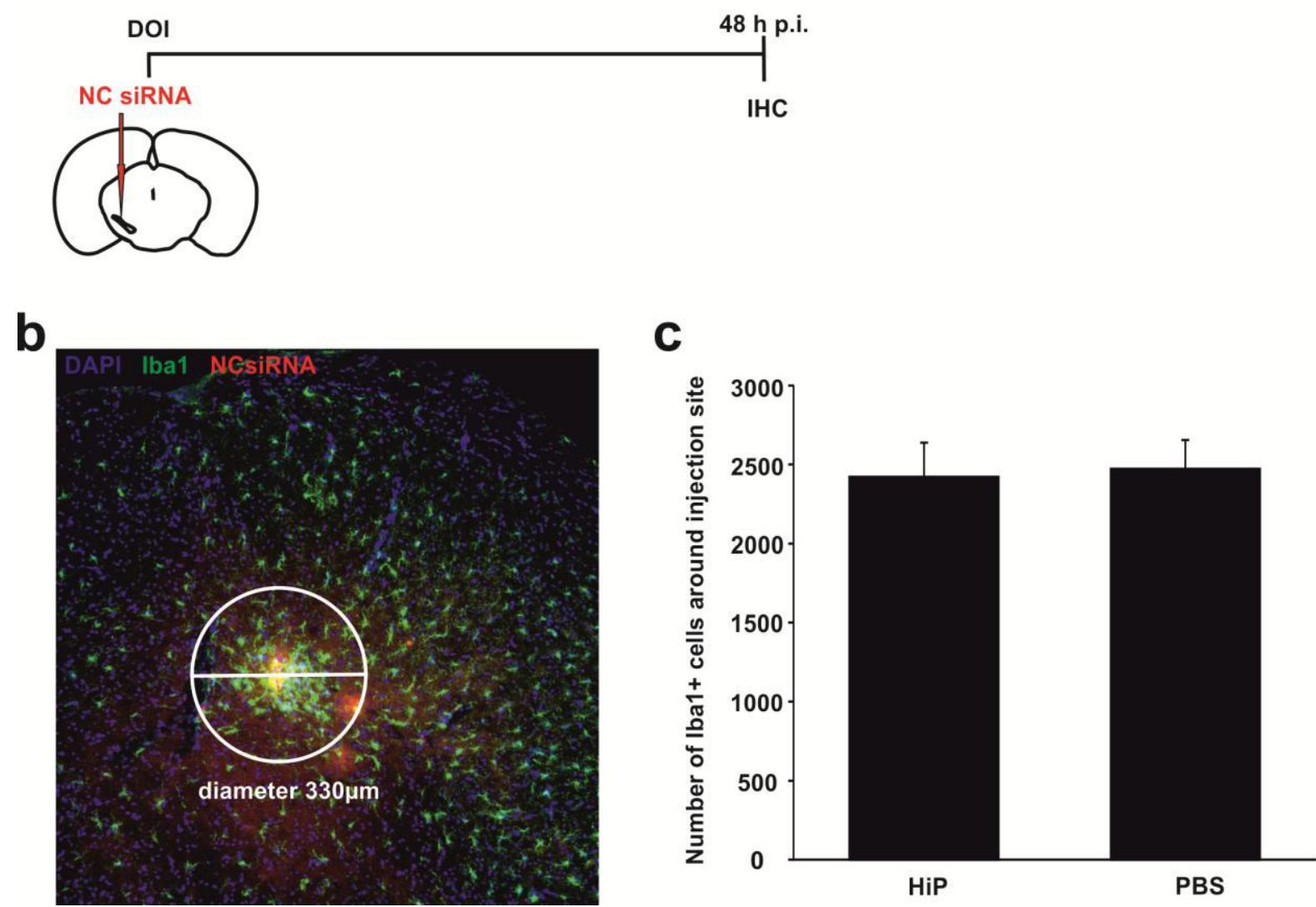

Figure 3.5/2: Transfection using a lipid-based transfection reagent is not increasing microglial invasion around the injection site. (a) Experimental layout. A fluorescently labeled NC siRNA mixed with PBS or HiP was injected in the right SN of 8 weeks old male C57BI/6J mice, $48 \mathrm{~h}$ post-injection animals were sacrificed and tissues prepared for IHC analysis of Iba1 + cells around the injection site (DOI = day of injection). (b) Exemplary micrograph of the SN injection site. Sections were stained for Iba1 and nuclear staining was performed using DAPI. The white circle $(Q 330 \mu \mathrm{m})$ is indicating the site for stereological counting of Iba1 + cells around the injection site. (c) Stereological quantification of Iba $1+$ microglial cells around the injection site for animals injected with NC siRNA + HiP or NC siRNA + PBS ( $n=4$ animals per condition).

\subsubsection{Injection of miR-182 and miR-183 mimics into the murine SNpc leads to a stable increase in miR-182 and miR-183 levels detected by qRT-PCR}

For the assessment of transfection efficiency and stability of miRNA mimics in vivo, 250 pmol miR-182 or miR-183 mimic or NC siRNA were injected in the SNpc of mice. $6 \mathrm{~h}$, $48 \mathrm{~h}$ and $7 \mathrm{~d}$ post-injection animals were sacrificed, total RNA from the substantia nigra was isolated and miR-182 and miR-183 levels were detected by qRT-PCR (figure 3.5/3 a). 
Six hours after nigral injection of miR-182 and miR-183 mimics a strong increase in miR-182 and miR-183 levels in the SNpc compared to NC siRNA injected animals was detected by qRT-PCR (figure $3.14 \mathrm{~b}$ and c first column; miR-182 $13.3 \pm 0.5 \log _{2} \mathrm{fc}$; miR-183 $\left.13.5 \pm 0.3 \log _{2} \mathrm{fc}\right)$. After $48 \mathrm{~h} \mathrm{miR-182}\left(9.8 \pm 0.2 \log _{2} \mathrm{fc}\right)$ and miR-183 (10.1 $\left.\pm 1.2 \log _{2} \mathrm{fc}\right)$ levels in miRNA mimic injected animals decreased slightly compared to animals $6 \mathrm{~h}$ postinjection and remained stable 7 days (miR-182 $10.0 \pm 1.5 \log _{2} \mathrm{fc} ; \mathrm{miR}-1838.0 \pm 0.3 \log _{2} \mathrm{fc}$ ) after injection. However, compared to NC siRNA injected animals the miR-182 and miR183 levels in miRNA mimic transfected mice were strongly increased even 7 days postinjection.

a
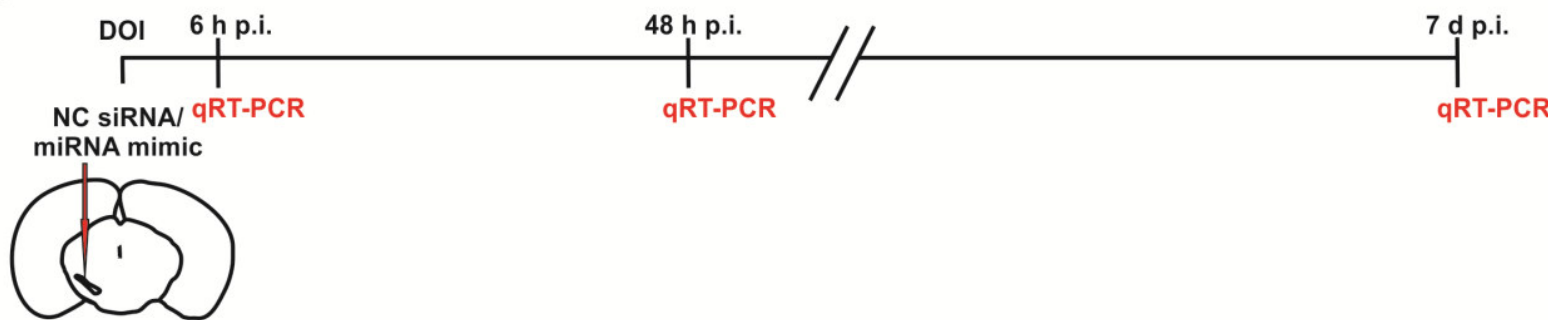

b

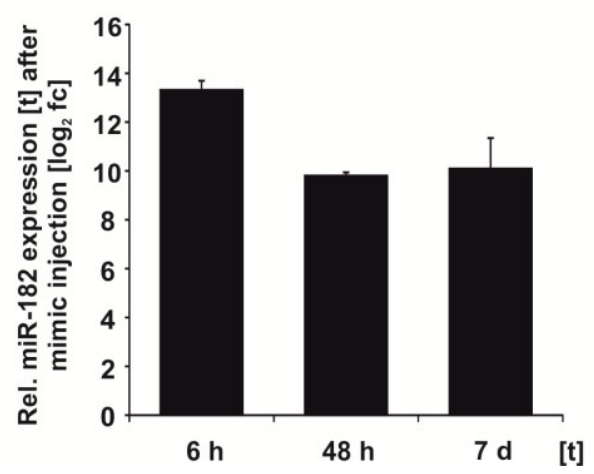

C

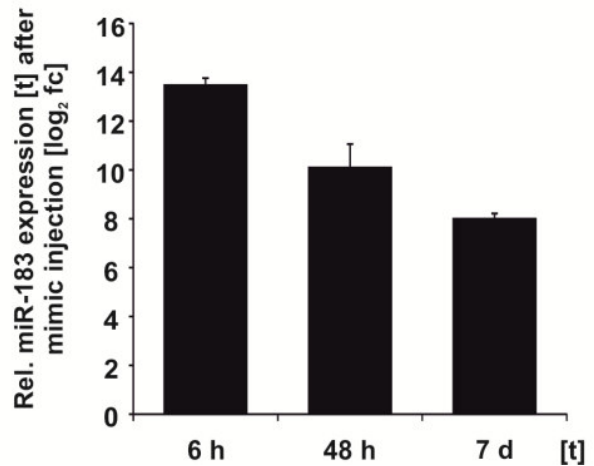

Figure 3.5/3: Increase in miRNA levels is stable several days after injection of synthetic miRNA mimics into the murine SN in vivo. (a) Experimental layout for analysis of transfection efficiency and stability. The respective synthetic miRNA mimic or NC (250 pmol) was injected in the right SN of 8 weeks old male C57BI/6J mice. $6 \mathrm{~h}, 48 \mathrm{~h}$ or $7 \mathrm{~d}$ postinjection animals were sacrificed, the midbrain containing the SN region was dissected and total RNA was isolated for qRT-PCR to evaluate miRNA expression levels. (b) and (c) Relative miR-182 (b) and miR-183 (c) expression levels 6 h, 48 $\mathrm{h}$ and $7 \mathrm{~d}$ after injection of miR-182 mimic or miR-183 mimic respectively ( $\mathrm{n}=3$ animals per condition; mean \pm SEM; data evaluation according to the $\Delta \Delta$ Ct-method). 


\subsubsection{Increased miR-182 levels lead to a higher survival of dopaminergic neurons in the SNpc of mice after MPTP intoxication}

In order to investigate the potential neuroprotective effects of increased miR-182 and miR-183 levels in an in vivo model for PD, animals received stereotactical injections of miR-182 or miR-183 mimics in the right SNpc. Control animals were injected with a NC siRNA. Beginning $48 \mathrm{~h}$ after stereotactical injection animals received intra-peritoneal MPTP (30 mg / kg body weight) injections at five consecutive days. At day 14 after the first MPTP injection animals were sacrificed and tissues were processed for stereological analysis of the SNpc dopaminergic neuron numbers as well as Nissl positive cells (figure 3.5/4 a).

After MPTP intoxication, NC siRNA injected animals showed a dramatic loss of TH positive dopaminergic neurons in the SNpc (figure 3.5/4 b $+c$ ). The stereological analysis of the ipsilateral SNpc resulted in a loss of about $45 \%$ in NC siRNA injected MPTP lesioned animals (NC + MPTP $8633.5 \pm 753.8 \mathrm{TH}+$ neurons per SN) compared to non-lesioned controls (PBS $15533.8 \pm 976.7 \mathrm{TH}+$ neurons per SN). Animals that were injected with 250 pmol of miR-182 mimic and intoxicated with MPTP showed a significantly higher number (14424.4 \pm 1165.8$)$ of TH-positive dopaminergic neurons in the SNpc than MPTP intoxicated animals injected with NC siRNA. A similar trend was observable in MPTPintoxicated mice that received an injection of miR-183 mimics $(11168.8 \pm 457.2 \mathrm{TH}+$ neurons), although these results were not significant.

In order to identify the total number of neuronal cells in the SNpc, Nissl staining was performed. The total number of neurons in the SNpc allows the conclusion if the loss of TH-positive dopaminergic neurons resulted from dopaminergic cell death or from a MPTP-induced downregulation of TH expression. As displayed in figure 3.5/4 d MPTP intoxication led to a severe loss of Nissl positive cells in the SNpc (PBS 16538.6 \pm 850.5 ; NC + MPTP $9015.8 \pm 833.6$ ) that was comparable to the loss of TH-positive dopaminergic neurons, indicating a robust dopaminergic neurodegeneration upon MPTP treatment. In miR-182 mimic injected MPTP-intoxicated animals the loss of Nissl positive cells was significantly less $(15815.9 \pm 1264.4)$ than in NC siRNA injected animals. Also in miR-183 injected animals (12742.3 \pm 688.9$)$ an attenuation of Nissl positive cell loss is detectable, although this was not statistically significant. 
a

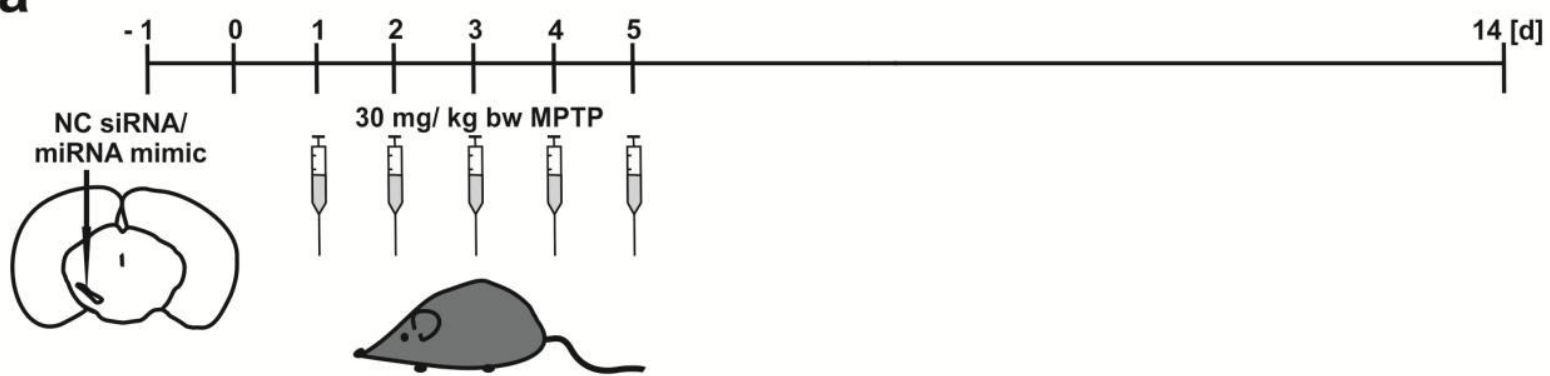

b

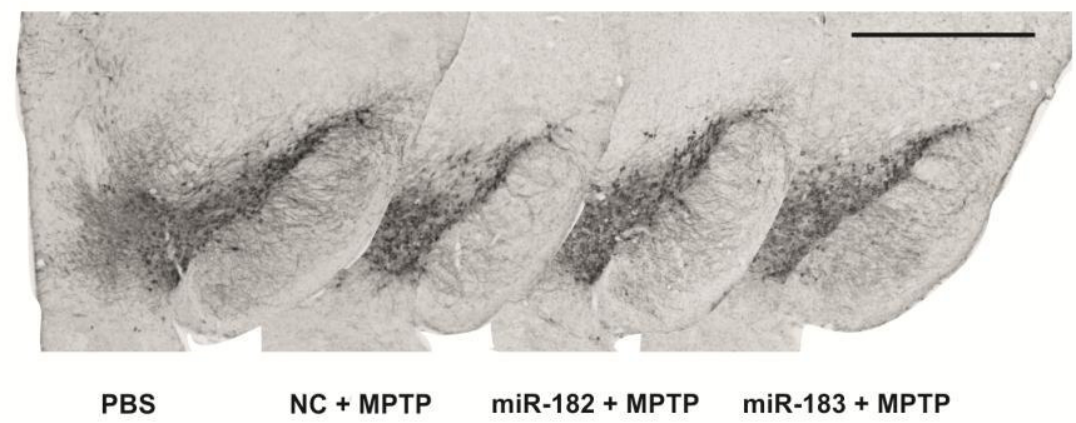

C

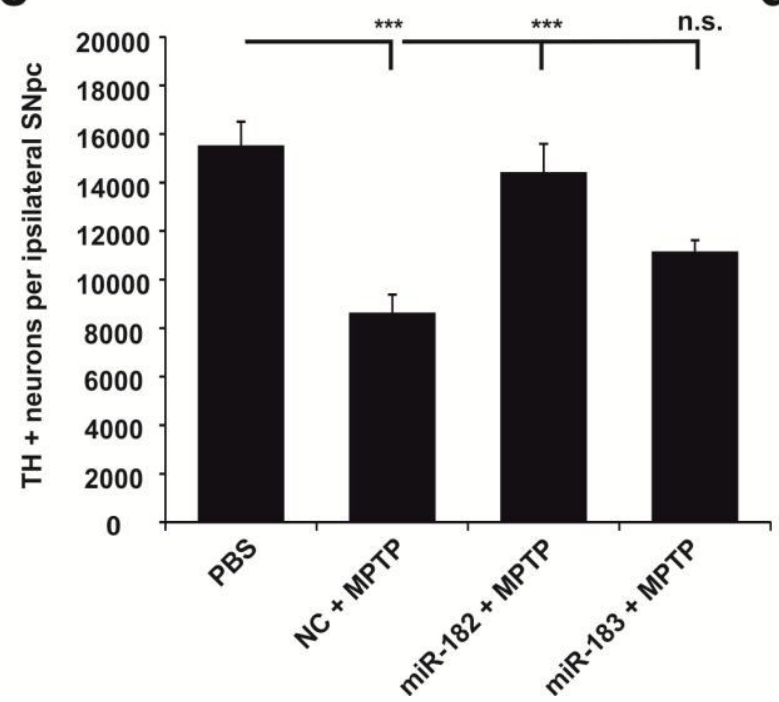

d

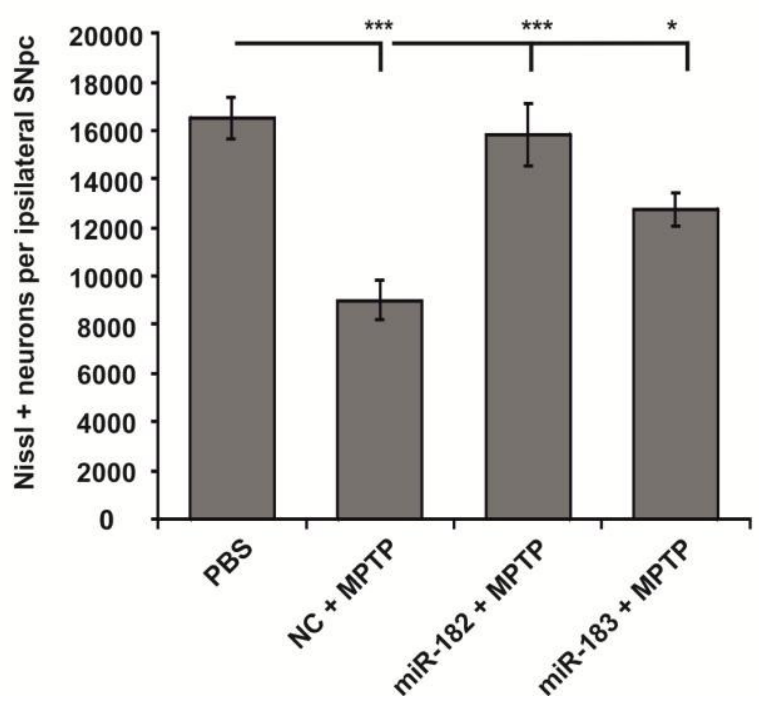

Figure 3.5/4: Increase in miR-182 levels leads to an increase in survival of dopaminergic TH+ neurons in the MPTP mouse model. (a) Experimental layout. 8 weeks old male C57BI/6J mice received a stereotactic injection of 250 pmol miRNA mimic or NC mixed with HiP (d-1) into the right SN. $48 \mathrm{~h}$ later (d1) intra-peritoneal MPTP injections (30 mg/ kg bw) were started and repeated for 5 consecutive days. $14 \mathrm{~d}$ after the first MPTP injection animals were sacrificed and tissues were harvested for IHC analysis. (b) Representative micrographs of SN sections of animals in experimental groups as indicated and immunolabeled against TH (scale bar $=1 \mathrm{~mm}$ ). (c, d) Quantitative stereological analysis of TH + (c) and Nissl + (d) neurons in the ipsilateral SNpc of experimental animals (PBS $n=5$, all other groups $n=6$; mean \pm SEM; one-way ANOVA with Dunnett's test post-hoc test). 
In non-MPTP treated animals the injection of NC siRNA or miRNA mimics did not lead to a change in TH positive dopaminergic neuron numbers (PBS: $15533.8 \pm 976.7$; NC siRNA + PBS: $15064.4 \pm$ 1357.6; miR-182 + PBS: $16504.9 \pm 1057.3 ;$ miR-183 +PBS: 16747.1 \pm 322.6 ) or Nissl positive neurons (PBS: $16538.6 \pm 850.5$; NC siRNA + PBS: $16279.4 \pm$ 1154.9; miR-182 + PBS: $17746.2 \pm 976.1 ; \operatorname{miR}-183$ +PBS: $18200.7 \pm 331.2 ; n=5$ in all groups).

\subsubsection{Increased nigral miR-182 levels lead to attenuated dopaminergic fiber loss and increased dopamine levels in the striatum of MPTP-intoxicated mice}

To investigate the potential protective effects of increased miR-182 and miR-183 levels on the nigral dopaminergic nerve terminals in the striatum after MPTP lesion, the density of DAT-positive dopaminergic fibers in the striatum was analyzed 14 days after the first MPTP injection (figure 3.5/5 a).

The representative micrographs in figure $3.5 / 5$ b show a strong reduction in DAT immunoreactive fiber density in the striatum of NC siRNA injected animals after MPTP lesion. In comparison to this the micrographs of miR-182 mimic injected animals show a less extensive reduction of dopaminergic fibers. A similar trend was observed in miR-183 injected animals. The quantification of DAT-positive fiber density (figure $3.5 / 5 \mathrm{c}$ ) showed a significant reduction of $57.0 \pm 6.8 \%$ in NC siRNA injected animals after MPTP lesion. Animals treated with miR-182 mimic showed significantly less fiber decline after MPTP treatment $(36.9 \pm 8.4 \%)$. A trend for decreased degeneration of dopaminergic fibers in the striatum was observed also in miR-183 injected animals, here $46.8 \pm 6.0 \%$ of the fibers degenerated, although this was not significant.

The injection of NC siRNA and miRNA mimics had no effect on striatal dopaminergic fiber density in animals that were not treated with MPTP (PBS: $100.0 \pm 6.6$ \%; NC siRNA + PBS: $102.2 \pm 11.1 \%$; miR-182 + PBS: $103.5 \pm 6.7 \%$; miR-183 + PBS: $102.7 \pm$ $2.3 \% ; n=5$ in all groups). 
a

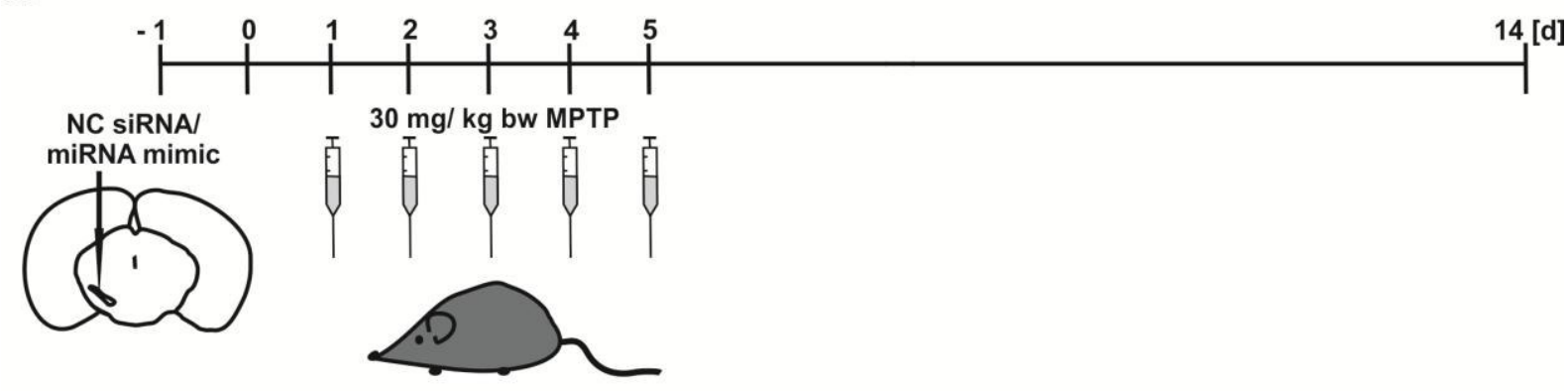

b

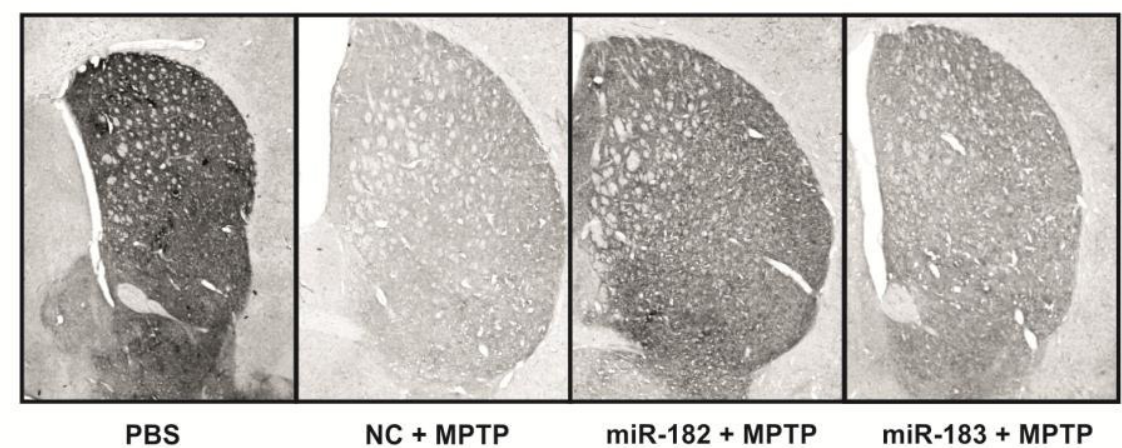

C

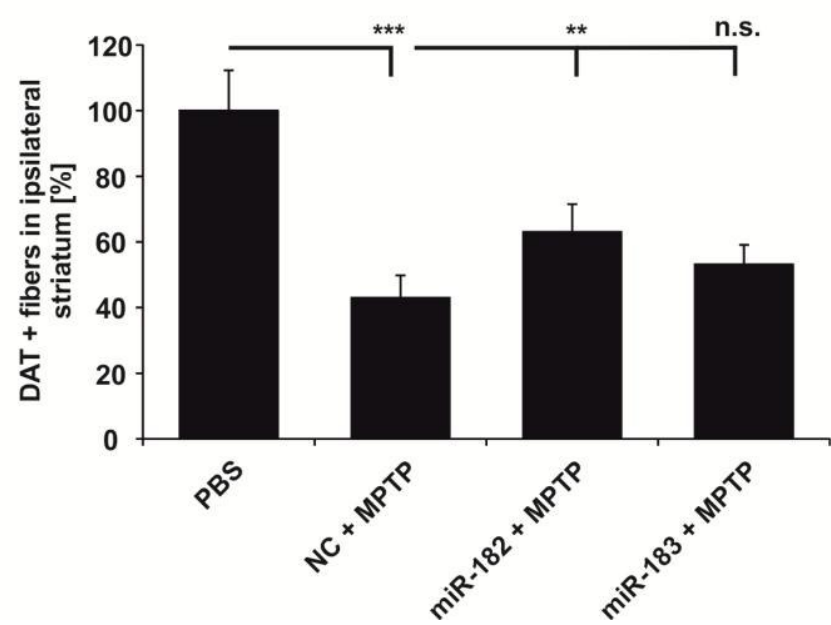

Figure 3.5/5: Increase in miR-182 levels leads to a higher dopaminergic striatal fiber density in the MPTP mouse model. (a) Experimental layout. 8 weeks old male C57BI/6J mice received a stereotactic injection of 250 pmol miRNA mimic or NC mixed with HiP (d-1) into the right SN. 48 h later (d1) intra-peritoneal MPTP injections (30 mg/ kg bw) were started and repeated for 5 consecutive days. $14 \mathrm{~d}$ after the first MPTP injection animals were sacrificed and tissues were obtained for IHC analysis. (b) Representative micrographs of striatal sections of animals in experimental groups as indicated and immunolabeled against DAT (scale bar $=1 \mathrm{~mm}$ ). (c) Relative quantification of DAT + fiber density normalized to PBS injected animals (PBS $n=5$, all other groups $n=6$; mean \pm SEM; one-way ANOVA with Dunnett's test post-hoc test).

In order to determine whether the observed morphological preservation of dopaminergic striatal fibers correlates with functional maintenance of these axonal nerve 
terminals the content of dopamine and its metabolites DOPAC and HVA in the striatum were measured by HPLC. To this, fresh striatal tissue was prepared 14 days after first MPTP injection to purify dopamine and its metabolites for neurochemical analysis. The results of the neurochemical analysis are displayed in figure 3.5/6. MPTP intoxication led in all experimental conditions to a massive decrease in striatal dopamine contents (figure 3.5/6 a). Compared to PBS injected control animals (18.0 $\pm 1.0 \mathrm{ng} / \mathrm{mg}$ wet tissue) striatal dopamine decreased about approximately $72 \%$ in NC siRNA treated and MPTP intoxicated mice (NC + MPTP: $5.1 \pm 0.2 \mathrm{ng} / \mathrm{mg}$ wet tissue). Striatal dopamine levels in miR-182 mimic-injected animals (miR-182 + MPTP: $6.7 \pm 0.4 \mathrm{ng} / \mathrm{mg}$ wet tissue) were significantly higher compared to NC siRNA treated animals. These results correlate partially with the morphological preservation of dopaminergic fibers in the striatum. In miR-183 mimic-injected animals (5.4 $\pm 0.2 \mathrm{ng} / \mathrm{mg}$ wet tissue) no difference in the dopamine levels compared to NC siRNA injected and MPTP treated animals could be observed.

a

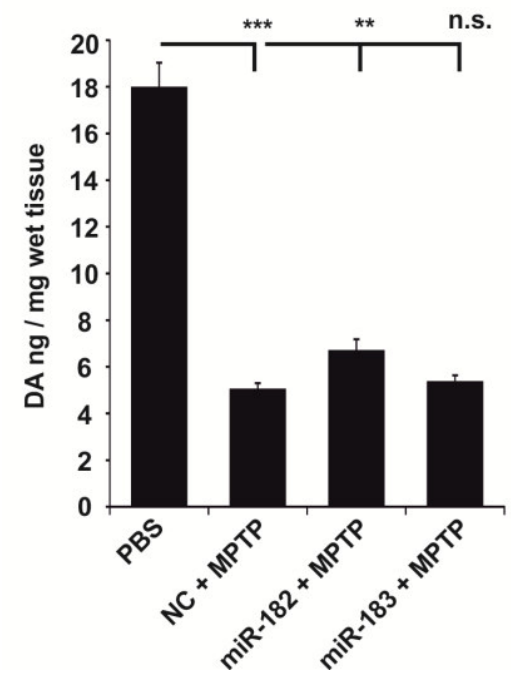

b

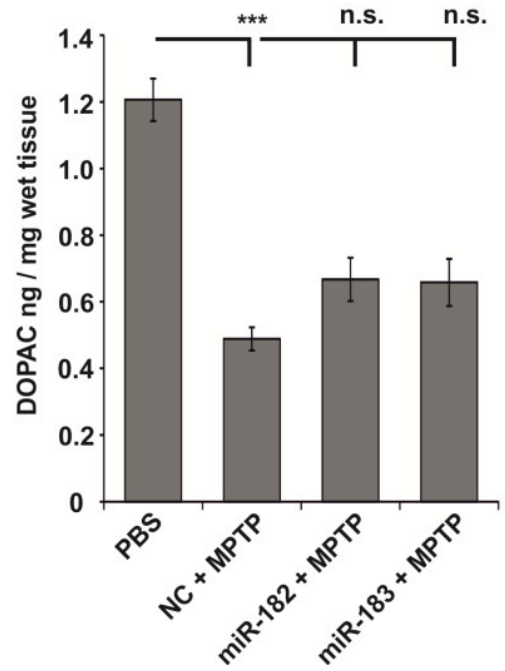

C

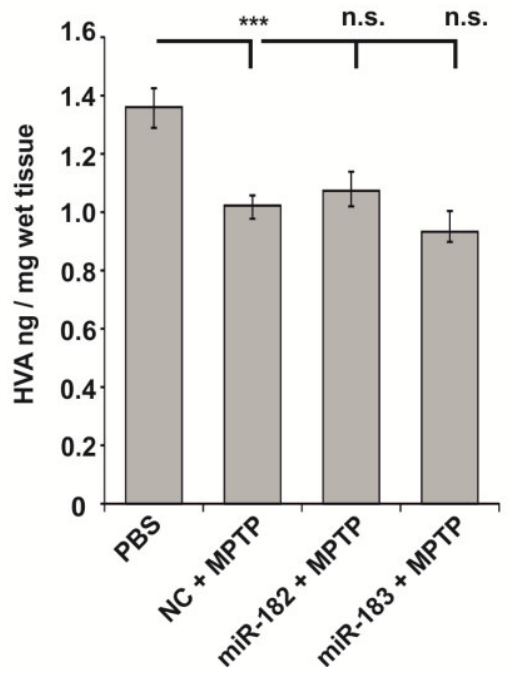

Figure 3.5/6: miR-182 increase leads to higher levels of striatal dopamine (DA) in the MPTP mouse model. Quantification of striatal DA (a), (3,4-dihydrophenylacetic acid (DOPAC) (b) and homovanillic acid (HVA) (c) contents in ipsilateral striata of experimental animals $($ PBS $n=5 ; N C+$ MPTP $n=9 ; \operatorname{miR}-182+$ MPTP $n=8 ; \operatorname{miR}-183+$ MPTP $n=9$; mean \pm SEM; one-way ANOVA with Dunnett's test post-hoc test).

Regarding the dopamine metabolites DOPAC and HVA a similar but not as pronounced depletion could be observed (figure 3.5/6 b +c). In NC siRNA injected MPTP 
intoxicated animals the striatal DOPAC levels decreased to $0.5 \pm 0.0 \mathrm{ng} / \mathrm{mg}$ wet tissue (PBS: $1.2 \pm 0.1 \mathrm{ng} / \mathrm{mg}$ wet tissue) as displayed in figure 3.5/6 b. Injection of miR-182 or miR-183 mimics had no significant effect on striatal DOPAC contents (miR-182 + MPTP: $0.7 \pm 0.1 \mathrm{ng} / \mathrm{mg}$ wet tissue; miR-183 + MPTP: $0.7 \pm 0.1 \mathrm{ng} / \mathrm{mg}$ wet tissue). Striatal HVA levels decreased in all MPTP treated groups (figure 3.5/6 c). Again miR-182 or miR-183 increase led to no significant differences in striatal HVA compared to NC siRNA treated animals (PBS: $1.4 \pm 0.1 \mathrm{ng} / \mathrm{mg}$ wet tissue; NC + MPTP: $1.0 \pm 0.0 \mathrm{ng} / \mathrm{mg}$ wet tissue; miR182 + MPTP: $1.1 \pm 0.0 \mathrm{ng} / \mathrm{mg}$ wet tissue; miR-183 + MPTP: $0.9 \pm 0.0 \mathrm{ng} / \mathrm{mg}$ wet tissue).

In animals not intoxicated with MPTP the nigral injection of NC siRNA or miRNA mimics had no effect on striatal dopamine, DOPAC or HVA contents (NC + PBS: $17.4 \pm 0.7$ ng dopamine / mg wet tissue; $1.5 \pm 0.1 \mathrm{ng}$ DOPAC / mg wet tissue; $1.3 \pm 0.1 \mathrm{ng} \mathrm{HVA} / \mathrm{mg}$ wet tissue; miR-182 + PBS: $16.9 \pm 0.7 \mathrm{ng}$ dopamine / mg wet tissue; $1.1 \pm 0.0 \mathrm{ng}$ DOPAC / mg wet tissue; $1.2 \pm 0.1 \mathrm{ng}$ HVA / mg wet tissue; miR-183 + PBS: $16.2 \pm 0.8 \mathrm{ng}$ dopamine / mg wet tissue; $1.2 \pm 0.1 \mathrm{ng}$ DOPAC / mg wet tissue; $1.3 \pm 0.1 \mathrm{ng}$ HVA / mg wet tissue; NC + PBS $n=5$; all other groups $n=6$ ).

\subsubsection{Increased miR-182 and miR-183 levels lead to a partial restoration of motor deficits}

In addition to the morphological and neurochemical analysis, animal behavior was assessed 13 days after the first MPTP injection. For analysis of motor coordination the cylinder rearing test was utilized. General motor behavior was tested in the open field test.

In the cylinder test the mouse natural exploratory behavior is used to study motor coordination. Not intoxicated animals (PBS) mainly performed free rears (45.5 $\pm 3.5 \%$ ) and rears assisted with both paws $(42.4 \pm 3.4 \%)$, whereas lateralized assisted rears were exceptions (left: $8.6 \pm 1.8 \%$; right: $3.5 \pm 1.9 \%$ ) as displayed in figure $3.5 / 7 \mathrm{~b}$ (black bars). In animals treated with NC siRNA and injected with MPTP these proportions changed significantly. NC siRNA-treated and MPTP-intoxicated mice showed an increase in rears assisted with both paws $(64.8 \pm 3.4 \%)$ and a corresponding decrease in free rears $(25.3 \pm$ 
3.3 \%). Left and right paw assisted rears were not significantly different from non-MPTP intoxicated animals (figure 3.5/7 b dark grey bars).

a

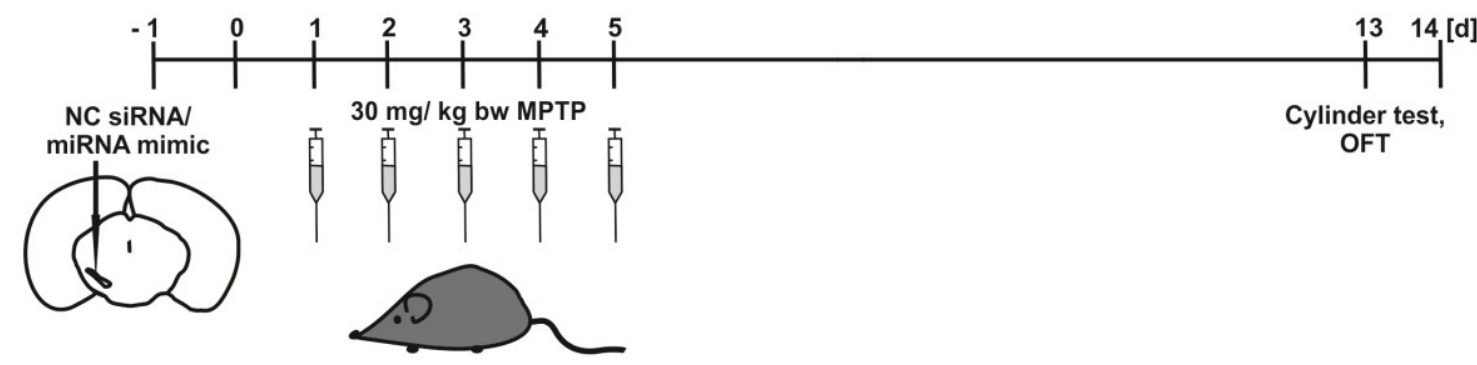

b

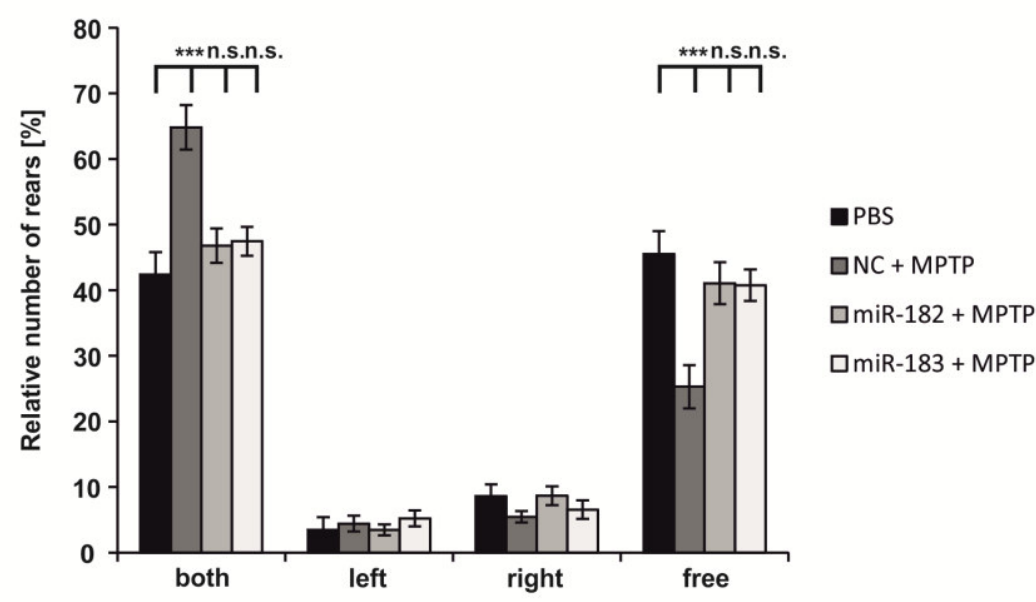

C

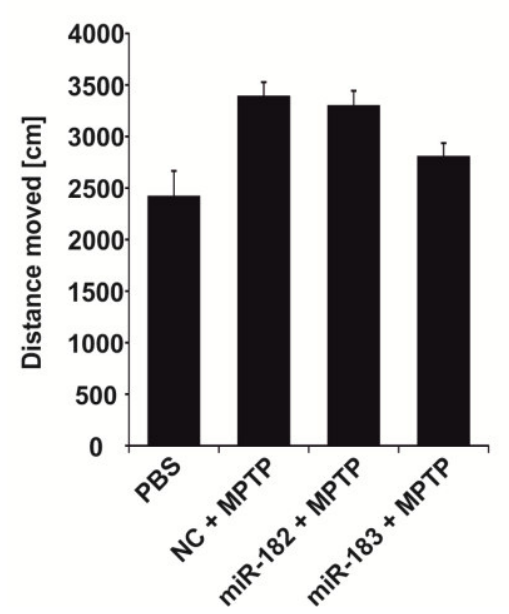

d

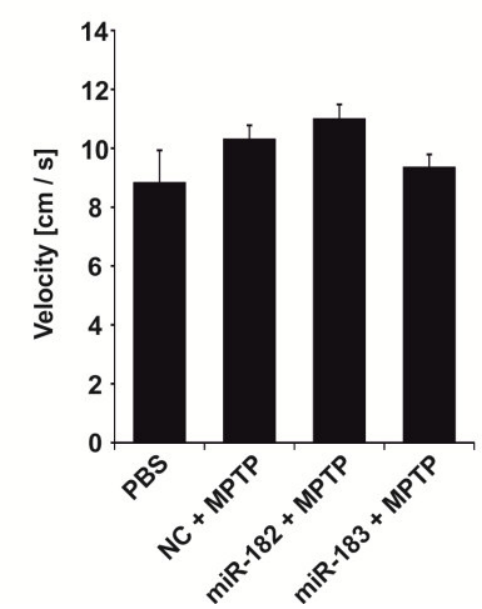

$\mathbf{e}$

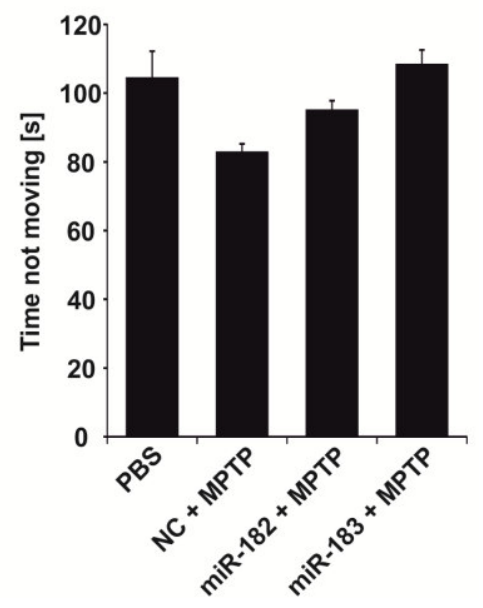

Figure 3.5/7: Effect of increased miR-182 and miR-183 levels on motor behavior in the MPTP mouse model. (a) Experimental layout. 8 weeks old male C57BI/6J mice received a stereotactic injection of 250 pmol miRNA mimic or NC mixed with HiP (d-1) into the right SN. $48 \mathrm{~h}$ later (d1intra-peritoneal MPTP injections $(30 \mathrm{mg} / \mathrm{kg} \mathrm{bw}$ ) were started and repeated on 5 consecutive days. On day 13 after the first MPTP injection the animals' motor behavior was tested in the cylinder test (coordination) and the open field test (OFT; general motor behavior). Animals were sacrificed on day 14 after the first MPTP injection. (b) Quantification of the proportion of rears the animals performed with the aid of both 
paws (both), the left (left) or the right (right) paw, and without the use of their paws (free). (c, d, e) General motor behavior was assessed using the OFT. (c) Quantification of the total distance the animal moved within the 5 min testing time. (d) Analysis of the mean velocity of the animals during the 5 min testing time. (e) Quantification of the average time the animals were not moving during the testing period (PBS $n=5$; all other groups $n=15$ animals per group; mean \pm SEM; one-way ANOVA with Dunnett's test post-hoc test).

Animals treated with miR-182 showed an effective restoration of the observed deficits in rearing behavior in NC siRNA-treated and MPTP-intoxicated animals (figure 3.5/7 b middle grey bars). After MPTP intoxication miR-182-treated animals performed $46.8 \pm 2.6$ $\%$ of the total rears with assistance of both paws and $41.0 \pm 3.2 \%$ as free rears without assistance. Thus, these animals did not perform significantly different from non-MPTP intoxicated animals (PBS). The left and right paw assisted rear numbers were similar to all other treatment groups (left: $8.7 \pm 1.4 \%$; right: $3.5 \pm 0.8 \%$ ). In miR-183 injected animals comparable results were observable. After MPTP intoxication they still performed $40.8 \pm$ $2.4 \%$ of the rears as free rears and $47.4 \pm 2.2 \%$ under assistance of both paws. Left paw assisted rears $(6.6 \pm 1.4 \%)$ and right paw assisted rears $(5.2 \pm 1.2 \%)$ occurred in similar proportions as in all other groups (figure 3.5/7 b light grey bars). miR-183 treated animals performed not significantly different than non-lesioned animals.

The injection of miRNA mimics or NC siRNA without intoxication with MPTP had no effect on the rearing behavior of the animals (NC siRNA: both $44.6 \pm 1.4 \%$; right $2.8 \pm$ $0.5 \%$; left $4.7 \pm 1.2 \%$; free $47.9 \pm 1.4 \%$; miR-182: both $47.9 \pm 2.9 \%$; right $3.9 \pm 0.9 \%$; left $7.3 \pm 1.5 \%$; free $40.9 \pm 3.3 \%$; miR-183: both $42.1 \pm 1.8 \%$; right $3.9 \pm 1.0 \%$; left $9.4 \pm 1.1$ $\%$; free $44.4 \pm 1.2 \%)$.

In the open field test the evaluation was focused on the parameters 'distance moved', 'velocity' and 'time not moving' (figure 3.5/7 c, d + e). Non-intoxicated animals (PBS) moved a distance of $2416.5 \pm 248.4 \mathrm{~cm}$ in total. MPTP intoxication and treatment with the NC siRNA led to no significant change in this parameter $(3385.8 \pm 145.1 \mathrm{~cm})$. In the miRNA mimic treated animals there was also no significant difference observable (miR-182 + MPTP: $3294.3 \pm 126.3 \mathrm{~cm} ;$ miR-183 + MPTP: $2800.8 \pm 115.9 \mathrm{~cm})$. Similar results were obtained for the parameters 'velocity' and 'time not moving', again no significant difference between treatment groups could be shown. On average, nonintoxicated animals (PBS) moved with a velocity of $8.8 \pm 1.1 \mathrm{~cm} / \mathrm{s}$. All MPTP-intoxicated animals showed similar velocities independent if treated with the NC siRNA or the miRNA 
mimics (NC + MPTP: $10.3 \pm 0.5 \mathrm{~cm} / \mathrm{s} ; \operatorname{miR}-182+$ MPTP: $11.0 \pm 0.5 \mathrm{~cm} / \mathrm{s} ; \mathrm{miR}-183 \pm$ MPTP: $9.3 \pm 0.4 \mathrm{~cm} / \mathrm{s})$. PBS injected animals spent on average $104.3 \pm 7.8 \mathrm{~s}$ without movement in the arena. This time was not significantly different in the MPTP lesioned animals independent of whether they received the NC siRNA or the miRNA mimics (NC + MPTP: $82.7 \pm 2.5$ s; miR-182 + MPTP: $95.0 \pm 2.8$ s; miR-183 + MPTP: $108.2 \pm 4.3$ s) Therefore, the lesion itself as well as the treatment with miRNA mimics or NC siRNA did not affect the chosen parameters in the open field test. 


\section{Discussion}

Today, PD is diagnosed with the appearance of motor symptoms; these occur late in disease progression when the majority of nigrostriatal projections are already degenerated. Other major obstacles in the treatment of PD are the lack of knowledge regarding the cause for the formation of the disease and missing treatment options that prevent further degeneration of the nigrostriatal system and induce regeneration of dopaminergic neurons. Although PD is a system disorder involving most parts of the brain, the malfunction of the nigrostriatal dopaminergic projections is of prime clinical importance. So far, several pathomechanisms for the degeneration of dopaminergic neurons have been proposed and studied; among them protein mishandling and aggregation, oxidative stress, mitochondrial dysfunction and energy failure. All mentioned mechanisms seem to be involved in PD pathogenesis, however, whether they are cause or consequence is still not known.

Since their discovery, miRNAs have been extensively studied and their importance for the development and maintenance of the CNS has become clear. Even though in recent years it was shown that miRNAs are also involved in the formation of neurodegenerative diseases, their role in PD is still under debate. Understanding miRNAdependent regulations in dopaminergic neuron biology and PD can provide valuable insights into PD pathogenesis and also might lead to new therapeutic strategies for neuroprotection and regeneration of the nigrostriatal system. The present work aims to elucidate miRNA expression changes in dopaminergic neurons upon development and neurite outgrowth, as well as in the degenerating and regenerating substantia nigra. Furthermore, it should be exploited if specific miRNAs have a neuroprotective or neuroregenerative potential in dopaminergic neurons in vitro and in vivo.

\section{1 miRNAs in maturation and neurite outgrowth of PMNs}

In order to analyze miRNA expression changes upon development and neurite outgrowth of PMNs, small RNA sequencing of PMN cultures at different time points of maturation was performed. Additionally, the effect of the pro-dopaminergic growth factor GDNF on miRNA expression in PMNs was evaluated. PMN cultures are mixed 
neuronal cultures containing 5-8\% dopaminergic neurons together with GABAergic and serotonergic neurons. Therefore, it is important to mention that the sequencing results do not reflect the miRNA expression changes of a pure dopaminergic neuron population, but represent the miRNA expression changes in developing midbrain neurons. We assume that the cellular mechanisms that drive neuronal fate and neurite outgrowth are global and thus similar among different neuronal subtypes. Therefore, the present study gives important insights into miRNA regulations upon neuronal development and neuron outgrowth. However, because the growth factor GDNF acts specifically on dopaminergic neurons and has no effect on the differentiation and survival of GABAergic or serotonergic neurons (Lin et al. 1993) it is very likely that the observed effects of GDNF on PMN miRNA expression are specific for dopaminergic neurons.

\subsection{1 miRNA expression in developing PMNs - major changes occur during early development}

Sequencing of small RNA libraries derived from PMN cultures at DIV 1, DIV 5 and DIV 10 detected 848 out of 1410 known murine mature miRNAs. Differential expression analysis revealed that the major changes in miRNA expression occur during early development of PMNs, between DIV 1 and DIV 5. Out of the 848 detected miRNAs the expression of 163 miRNAs was significantly regulated between DIV 1 and DIV 5, with a very pronounced regulation ( $\geq 4$-fold) of 47 miRNAs. The massive regulation in miRNA expression between DIV 1 (E 13.5) and DIV 5 (E 17.5) and the lack of major miRNA expression changes between DIV 5 and DIV 10 (P 0 ) indicate that the fate of PMNs including dopaminergic neurons is already determined at DIV 5. This is confirmed by different studies analyzing differentiation and maturation of rodent midbrain dopaminergic neurons in vitro and in vivo (reviewed in Perrone-Capano \& Porzio 2000). Perrone-Capano \& Porzio propose a model in which differentiation (requiring global changes in gene expression) of dopaminergic midbrain neurons occurs between E 11 and E 14, whereas the final maturation (requiring moderate 'fine tuning') takes place between $E 15$ and E 18. These findings were confirmed by analyses of DA uptake and expression of specific transcription factors. The importance of miRNAs for the final maturation of dopaminergic neurons was also shown by Kim and colleagues in 2000, where Dicer 
depletion led to strongly reduced numbers of dopaminergic neurons differentiated from ES cells whereas other neuron classes like GABAergic neurons were not affected in the same extent. This phenotype could be rescued by transfection of the small RNA fraction isolated from rodent embryonic midbrains. The target prediction analyses for regulated miRNAs between DIV 1 and DIV 5 and the functional annotation of these target genes also indicate important effects of miRNA expression changes on PMN differentiation and maturation. Taken together, the major changes in miRNA expression occur during differentiation of PMNs, whereas maturation and neurite outgrowth require milder changes in miRNA expression.

\subsubsection{The regulated miRNAs are important for neuronal differentiation and function}

Among the highly regulated miRNAs between DIV 1 and DIV 5 (with more than 500 reads per condition) there are several miRNAs that are known to play a role in neuronal development. miR-92a is down regulated at DIV 5 and is important for neural stem cell expansion (Bian et al. 2013). Beveridge and colleagues could show that neuronal differentiation lead to a down-regulation of the miR-17 family including miR-92a (Beveridge et al. 2009), underlining the present results that show that miR-92a is down regulated in differentiated PMNs. In contrast to this decreased expression of miRNAs involved in differentiation, there are several miRNAs significantly up-regulated in PMNs at DIV 5 that play a role in neurite growth, spine formation and synaptic plasticity. Among them is miR-138; a miRNA that is involved in regulation of neurite outgrowth by targeting SIRT1 mRNA (Liu et al. 2013). Interestingly, Shi and colleagues could demonstrate that miR-138 is also involved in synaptic plasticity, as it regulates synaptic Lypla1 protein levels (Shi et al. 2010). Another miRNA important for neurite growth, spine formation and synapse formation and function is miR-132 that is up regulated in PMNs at DIV 5. miR-132 overexpression lead to an increase in dendritic branching and increased dendritic protrusion width in primary hippocampal cultures (Edbauer et al. 2010). Pathania and colleagues could show that miR-132 enhances dendritic morphogenesis, synaptic integration and survival of newborn olfactory bulb and hippocampal neurons (Magill et al. 2010; Pathania et al. 2012). Equally up-regulated at DIV 5 are the two miRNAs from the 
miR-129 family: miR-129-5p and miR-129-2-3p. The precise functions of these miRNAs are not fully understood yet, but it is known that they play a role in cell proliferation, are important for synaptic functions and neuronal excitability (Cao et al. 2012; Sosanya et al. 2013; Wu et al. 2014). There are also indications for a dysregulation of these miRNAs in neurodegenerative diseases like $A D$ and $P D$, even though their role in the formation of these diseases is not elucidated yet (Briggs et al. 2015; Satoh 2012). The up-regulated miR-7b has been shown to regulate neurite length in developing hippocampal neurons and controls the growth of the cortex in mice (Pollock et al. 2014; van Spronsen et al. 2013). Interestingly, other miRNAs known to be regulated in neuronal development and function were not significantly regulated in the present study. There is evidence that miR9 and miR-124 expression is regulated during neuronal differentiation (reviewed in Gao 2010). However, in the present study the expression of those miRNAs was not significantly altered. One possible explanation is that the selected time intervals were too long to detect more subtle changes. It also might be that the effects of these miRNAs in PMNs are exerted at earlier time points during development.

In order to find potential candidate miRNAs that might enhance neuroregeneration and act neuroprotectively among the regulated miRNAs in this study $I$ performed target prediction analyses and functional annotation of these target genes. Additionally, I searched for already experimentally validated target genes. Based on these analyses, a list with appropriate candidate miRNAs was assembled; these miRNAs will be tested for their effects in PMNs in other experiments that are not part of this thesis.

\subsubsection{GDNF treatment leads to a specific regulation of miR-182 and miR-183 expression}

In order to analyze the influence of the pro-dopaminergic growth factor GDNF on miRNA expression in PMNs, a small RNA sequencing was performed. The small RNA sequencing and differential expression analyses comparing the miRNA expression in GDNF-treated PMN cultures with control-treated PMN cultures showed that out of the 848 detected miRNAs only two were significantly changed in their expression levels upon GDNF treatment. At DIV 5, miR-182 and miR-183 expression was significantly higher in GDNF-treated cultures than in control-treated PMNs, indicating that in untreated cultures the miR-182 and miR-183 expression declines with time. In order to verify the sequencing 
results we performed $\mathrm{qRT}-\mathrm{PCR}$ to determine the miR-182 and miR-183 expression in GDNF and control-treated PMNs at DIV 1, DIV 3, DIV 5 and DIV 7. In contrast to the sequencing data, the qRT-PCR showed that GDNF treatment lead to a constant increase in miR-182 and miR-183 expression at all time points, including DIV 1. The very specific effect of GDNF on the miRNAome of PMNs indicates that the regulated miRNAs are involved in mediating the beneficial effects of GDNF on dopaminergic neurons. Furthermore, it could be speculated that the regulation of these miRNAs is specific for dopaminergic PMNs as GDNF acts particularly on dopaminergic neurons. In order to even further verify this hypothesis it would be necessary to perform miR-182 and miR-183 expression analyses exclusively from GDNF-treated dopaminergic PMNs. Therefore, dopaminergic neurons would have to be isolated from other neuronal cells in the mixed PMN culture. This, however, is difficult to achieve, because cell sorting of adherent cultures requires trypsinization; a process that is very likely to change gene expression and thus also miRNA expression. Another possibility would be single cell PCR, a technique that allows the isolation and expression analysis of single cells. Recently, the first kits were developed that allow miRNA expression analysis from single cells or with minimal RNA amounts. These might give insight if the GDNF-mediated increase of miR-182 and miR-183 is specific for dopaminergic neurons.

A mutual regulation of neurotrophic factors and miRNAs has been previously reported, even though the finding that GDNF regulates the expression of certain miRNAs in neurons is new. There is only one previous study, which reports that acute treatment with high doses of GDNF leads to a differential expression of miRNAs in 6-OHDA treated MN9D cells (Li et al. 2013). The effects of chronic GDNF treatment on miRNA expression in neurons are not discussed there and the results are not comparable to the present study, because of a totally different methodology and the use of the mouse neuroblastoma fusion cell line MN9D, which is immortalized and thus its comparability to primary neurons is very limited. One of the best studied examples for neurotrophin regulated miRNA expression is the interaction between brain derived neurotrophic factor (BDNF) and the miR-212/132 cluster. A deep sequencing experiment revealed that BDNF induces the expression of the miR-212/132 cluster via the ERK1/2 pathway in primary cortical neurons (Remenyi et al. 2010). These findings led to the assumption that some of the effects of BDNF are mediated by these miRNAs, which were known to be important 
regulators of neurite growth, dendritic development and synapse function. This hypothesis was supported by the discovery that MeCP2 expression, a protein that is crucial for neuronal development, is regulated by miR-132 and miR-212. Interestingly, MeCP2 takes part in the control of BDNF expression, thereby creating a feedback loop for homeostatic control of MeCP2, BDNF and miR-132/miR-212 respectively (Im et al. 2010; Klein et al. 2007). Another example for neurotrophin-mediated miRNA expression is the induction of miR-221 and miR-222 by nerve growth factor (NGF) due to sustained activation of ERK1/2 (Terasawa et al. 2009). In both mentioned examples neurotrophininduced miRNA expression is mediated via increased ERK1/2 signaling. GDNF can also activate the ERK1/2 pathway which in turn activates several transcription factors (Stamboulian et al. 2010; Yang et al. 2001), among them SP1, a transcription factor that was shown to mediate expression of miR-182 and miR-183 (Yang et al. 2014). Thus, it is possible that GDNF induces miR-182 and miR-183 expression by activation of ERK1/2 and subsequent phosphorylation of SP1. However, by now there is no experimental evidence for this hypothesis. Nevertheless, there is accumulating evidence that miRNAs and neurotrophic factors regulate each other, a fact that is of major importance for the understanding of neuronal development, function and maintenance.

\section{2 miRNA expression changes in the degenerating and regenerating murine $\mathrm{SN}$}

In order to reveal the miRNAome of the midbrain and analyze the changes in miRNA expression levels upon degeneration and regeneration of $\mathrm{SN}$ dopaminergic neurons in vivo, the unilateral striatal 6-OHDA mouse model was employed and small RNA sequencing of the midbrain was performed 4 weeks (degeneration) and 12 weeks (regeneration) after intrastriatal injection. By small RNA sequencing 780 mature miRNAs were detected in the murine midbrain. In contrast to a deep sequencing analysis of the whole mouse brain where miR-9 was found to be most abundant (Chiang et al. 2010), in the unlesioned murine midbrain miR-181a showed the highest expression. Nonetheless, also other abundant brain miRNAs like miR-127, miR-9, miR-99b and miR-125b were expressed at high levels in the murine midbrain. 
Four weeks after intrastriatal 6-OHDA application - at the time point when the degeneration of the nigrostriatal system is at its maximum - out of the 780 detected miRNAs 5 showed significantly changed expression levels. Upon spontaneous recovery 12 weeks after the striatal 6-OHDA lesion the expression of 12 miRNAs was significantly regulated. Even though the analysis was done with total midbrain samples, the observed miRNA expression changes are very specific, indicating that the regulated miRNAs play important roles upon degenerative and regenerative processes of $\mathrm{SN}$ dopaminergic neurons. Additionally, it has to be mentioned that the midbrain used for the analysis naturally contains all types of neuronal and glial cells. Thus, it is possible that the observed changes in miRNA expression derive from different cell types, although the striatal 6-OHDA application excludes a mechanical lesion of the substantia nigra and the selective catecholaminergic toxicity of 6-OHDA-induced retrograde axonal degeneration imply a predominant lesion of dopaminergic neurons in the SNpc.

\subsection{1 miRNA expression changes in the degeneration model}

Only $0.6 \%$ of all detected miRNAs were regulated 4 weeks after striatal 6-OHDA injection. This reflects that the analysis was done at a time point were the degeneration is maximal and not in the acute phase shortly after the 6-OHDA injection, where a much stronger regulation might take place. Thus, this very specific pattern indicates that the regulated miRNAs play a role in progressive degeneration of dopaminergic neurons. From the 5 miRNAs with differential expression levels in the midbrain miR-501-3p, miR-100 and miR-331-3p were significantly up-regulated, whereas miR-486 and miR-3107 showed a decreased expression. The functional annotation of the target genes of these miRNAs revealed that they are important regulators of the MAPK, Wnt and mTor signaling pathways, reflecting the impact of 6-OHDA on the physiology of dopaminergic neurons. Until now there is no other study published that investigates miRNA expression in a mouse model for PD, neither for 6-OHDA nor for MPTP. Thus, the present data cannot be compared to other studies. Even the effect of the mentioned neurotoxins on PMNs or other neuronal cultures with respect to miRNA expression is not studied so far. There is one study published analyzing the effect of 6-OHDA on miRNA expression in MN9D cells, 
but this is obviously not comparable to the present data and none of miRNAs reported to be regulated in this paper (Li et al. 2013b) were regulated in our study.

Some of the regulated miRNAs have been reported to be involved in neurodegeneration in other contexts than PD. The up-regulated miR-501-3p is also increased in animals infected with Venezuelan equine encephalitis virus (VEEV), a virus that primarily affects neurons and glial cells and results in neurodegeneration (Bhomia et al. 2010). Interestingly, miR-501-3p as well as miR-100 and miR-331-3p were also increased in a mouse model for $A D$, here the authors discuss the function of this regulation as maintenance of cell homeostasis (Luo et al. 2014). miR-100 is also upregulated in the cerebellum and the medial frontal gyrus of $A D$ patients (Cogswell et al. 2008) and miR-331-3p levels are elevated in a mouse model for prion-induced neurodegeneration (Saba et al. 2008). In the present study miR-486 levels are decreased in the SN 4 weeks after 6-OHDA lesion, this is interesting, because it was shown that upregulation of miR-486 prevents neurons from regeneration in spinal cord injury by repression of NeuroD6, a factor that protects neurons from oxidative stress (Jee et al. 2012). Thus, decreased miR-486 levels in our degeneration model might lead to protection from oxidative stress by increased NeuroD6 expression, which is important as 6-OHDA toxicity is mediated via ROS formation. The comparable miRNA expression changes in different models for neurodegeneration and different neurodegenerative diseases indicate once more that the molecular mechanisms underlying neurodegeneration are similar, independent from the affected neuron type and the underlying disease.

Unfortunately, there are only few studies analyzing global miRNA expression changes in brains of PD patients and none of them reports changes in expression levels of the miRNAs that were regulated here. However, as miRNA analysis from post-mortem samples is difficult, it is not clear if the results are comparable to well-controlled animal studies. 


\subsection{2 miRNAs involved in spontaneous recovery of SNpc dopaminergic neurons after 6- OHDA lesion}

In the regeneration model 12 weeks after the striatal 6-OHDA lesion 12 out of 780 miRNAs were significantly regulated in the midbrain. Thereof three miRNAs were upregulated, including miR-132*, miR-128-1* and miR-146b, whereas 9 miRNAs were downregulated. Among the down-regulated miRNAs were miR-100, which is up-regulated upon degeneration, miR-199a, miR-199b and miR-99a. The KEGG pathway analysis of the target genes of these miRNAs revealed that especially proteins involved in growth-associated pathways as ErbB signaling pathway, glioma and neurotrophin signaling pathway are affected by the regulated miRNAs; pathways that are likely to play a role in a regenerative response. So far, except for a few reports on spinal cord injury and stroke there are no studies investigating the role of miRNAs in the spontaneous recovery of central nervous system structures. However, some of the regulated miRNAs in the present study have been previously reported to be involved in neuroregenerative and growth-associated processes. The up-regulated miR-146b targets interleukin-1 receptor-associated kinase 1 (IRAK1), a kinase that regulates a pro-inflammatory response, and thus might prevent excessive inflammation of brain tissue, which is also present in the SN of PD patients (Hirsch \& Hunot 2009; Urdinguio et al. 2010). This indicates a primary role of this miRNA in glial and especially microglial function. In the neuronal context, an increase of this miRNA is associated with neurogenesis and neural stem cells (Liu et al. 2011; Stevanato \& Sinden 2014). It is interesting that miR-100, a miRNA up-regulated in the degeneration model, is down-regulated with spontaneous recovery. One miR-100 target is mTOR, a kinase that has different functions in cell growth, autophagy, protein synthesis and transcription (Torres et al. 2012). There is evidence that inhibition of the mTOR pathway is neuroprotective in acute toxin-induced models for PD (Malagelada et al. 2010); elevated miR-100 levels leading to a decrease in mTOR protein levels might be involved in this neuroprotective mechanism. In contrast, it has been shown that increased mTOR levels and activity, as expected with decreased miR-100, are promoting axon regeneration in the CNS (Park et al. 2008). Interestingly, also two other down-regulated miRNAs in the regeneration model, miR-199b and miR-99a, target mTOR (Torres et al. 2012). This indicates that the spontaneous regenerative response of the nigrostriatal system in the 6OHDA mouse model is mediated by elevated mTOR levels. Another target of miR-199b 
and miR-99a is sirtuin1 (Sirt1) (Saunders et al. 2010). Sirt1 is a NAD-dependent histone deacetylase (HDAC), which in neurons is involved in neuroprotection, longevity and axon growth. Decreased levels in miR-199b and miR-99a might lead to an increase in Sirt1 protein levels. There is accumulating evidence that increased Sirt1 levels and activation promote CNS axon regeneration and protect against neurodegeneration in vivo and in vitro (Cho \& Cavalli 2014; Kim et al. 2007; Li et al. 2013; Liu et al. 2013).

Taken together, the present data suggests that the spontaneous recovery in the 6OHDA mouse model for PD 12 weeks after striatal lesion could be mediated by a decreased microglial inflammatory response through increased miR-146b levels and increased mTOR and Sirt1 levels through decreased miR-100, miR-199b and miR-99a levels. In order to prove this hypothesis further studies analyzing the expression levels of the discussed proteins in the midbrain of this model are necessary.

\subsection{Effects of increased miR-182 and miR-183 levels on dopaminergic PMNs}

The manipulation of miRNA levels in cell culture by transfection of either synthetic miRNA mimics or miRNA inhibitors is a promising tool for the functional analysis of specific miRNAs. The small RNA sequencing of GDNF treated PMNs revealed the specific up-regulation of miR-182 and miR-183 upon GDNF treatment. miR-182 and miR-183 belong to the miR-96 182 183 cluster, an intergenic miRNA cluster that is located on the murine chromosome 6 and leads to the simultaneous expression of miR-96, miR-182 and miR-183. The regulation of miR-182 and miR-183 by GDNF indicates that GDNF might execute some of its effects via miR-182 and miR-183. The functional annotation of predicted target genes of these miRNAs also suggests that they might be involved in GDNF-mediated effects like neuroprotection and survival of dopaminergic neurons. PMN cultures were transfected with synthetic miR-182 or miR-183 mimics or NC siRNA, followed by analyses of neurite length, neurite regeneration and neuroprotection in different experimental setups. As mentioned before, PMNs are mixed neuronal cultures and the miRNA mimic transfection affected all present cell types, but for the analyses only dopaminergic neurons were included. Thus, the observed effects are experimentally validated for dopaminergic PMNs, but the possibility that also other neuronal cell types are affected cannot be excluded and need further investigation. Nevertheless, GDNF acts 
in a highly specific manner on dopaminergic neurons and thus the initial GDNF-mediated up-regulation of miR-182 and miR-183 is very likely to occur specifically in these cells.

\subsubsection{Increased miR-182 and miR-183 levels lead to longer neurites and increased neurite regeneration in dopaminergic PMNs}

Transfection of PMN cultures with synthetic miR-182 or miR-183 mimics led to an increased neurite growth and regeneration of dopaminergic neurons. Interestingly, the effect of the miRNA mimics was comparable to the effect of GDNF treatment in NC siRNA transfected cells. This indicates that miR-182 and miR-183 are mediating at least some of the beneficial effects of GDNF. The possibility that the observed increase in neurite length and regeneration was caused by the mere transfection of miRNA mimics can be excluded, because transfection of PMNs with miR-1a-3p mimics, a miRNA that was previously shown to have no effect on neurite growth, did not lead to longer neurites or increased neurite regeneration in dopaminergic neurons. In general, the effects of miR-182 mimics were a little more pronounced than the effects of miR-183 mimic transfection. Another noticeable fact is that a combined transfection of miR-182 and miR-183 mimics did not lead to a cumulative effect. One possible explanation for this is a saturation of the miRNA machinery like dicer (Castanotto \& Rossi 2009), another possibility is the mutual regulation of miR-182 and miR-183 mimic-mediated effects. As the neurite length in all experimental conditions increased between $70.4 \%$ and $83.9 \%$ compared to NC siRNA transfected cells one could also speculate that there might be a physiological limit to neurite outgrowth in dopaminergic PMNs, e.g. by limited growth substrate supply or speed of substrate production.

Until now there is no study published reporting the influence of miR-182 and miR183 on neurite outgrowth of dopaminergic neurons. Nevertheless, inhibition of the miR182 target genes Foxo3 and Foxo1 as well as the miR-183 target gene Taok1 are reported to be involved in neurite outgrowth (Gu et al. 2014; Schubert et al. 2003; Tavares et al. 2013; Wang et al. 2013). The present work shows a decrease in Foxo3 and Foxo1 expression in miR-182 mimic transfected PMN cultures. Interestingly, also in GDNF treated and miR-183 transfected PMNs a similar decrease in Foxo protein levels can be observed. It was previously reported for neuroblasts and enteric neurons that GDNF leads 
to a decrease in Foxo1 and Foxo3 protein expression and activation (Anitha et al. 2006; Srinivasan et al. 2005). In contrast, Foxo1 until now was not validated as a target for miR183 in mice, but in humans. Thus, it is very likely that miR-182 and miR-183 induced increase in neurite length is mediated among others via decreased expression of Foxo transcription factors. There was a trend of decreased Taok1 protein levels in miR-183 transfected as well as in GDNF treated PMNs, even though this did not reach statistical significance. This indicates that also a decrease in Taok1 protein levels might be involved in the miR-182/miR-183/GDNF mediated increase in neurite length and regeneration. Additionally to the direct targets of miR-182 and miR-183 the levels and activation of different growth-associated proteins were analyzed. Transfection of PMNs with miR-182 and miR-183 mimics led to no changes in Akt levels. Nevertheless, the increase in miR-182 and miR-183 levels resulted in a significantly higher amount of pAkt in PMNs, giving evidence for an increased activation of the PI3K-Akt pathway. A similar trend was observed in PMNs treated with GDNF. Furthermore, our results show a trend for an increased activation of the Mapk pathway in miR-182, miR-183 and GDNF treated cells, shown by the increased phosphorylation of Erk1 and Erk2. Again, the unphosphorylated isoforms were not affected by the different treatments. It was previously shown that GDNF leads to an increased activation of PI3K-Akt and Mapk signaling in dopaminergic cells and that this is required to mediate the GDNF effects (Carnicella et al. 2008; Pong et al. 2002). Both pathways are involved in mediating neurite growth and regeneration, thus it is not surprising that the increased neurite length and regeneration in miR-182 and miR183 transfected dopaminergic PMNs are accompanied by increased PI3K-Akt and Mapk signaling pathway activity, although the relation between miR-182/miR-183 and increased Akt and Mapk activity is not described in the literature yet. Interestingly, the increased Akt activation might lead to an additional inhibition of Foxo transcription factors by phosphorylation, since Foxo is a downstream target of the PI3K-Akt signaling and thus amplifies the miRNA-mediated effects (Brunet et al. 2001). In miR-182 and miR183 mimic transfected cells an increase in $\mathrm{Bcl}-2$ and phospho-S6 proteins was observable that can also be explained by an increase in PI3K-Akt signaling, since Bcl-2 and mTor (upstream of phospho-S6) are both downstream targets of Akt. The present data therefore suggest that the observed effects on neurite length and regeneration in 
dopaminergic PMNs are mediated via down-regulation of Foxo transcription factors and increased signaling via the PI3K-Akt and Mapk pathways.

\subsubsection{Increased miR-182 and miR-183 levels protect dopaminergic PMNs from MPP ${ }^{+}$ induced degeneration}

In order to validate if increased miR-182 and miR-183 levels protect dopaminergic $\mathrm{PMNs}$ from $\mathrm{MPP}^{+}$induced toxicity in the same way as GDNF, $2 \mu \mathrm{M} \mathrm{MPP}^{+}$were applied 24 $\mathrm{h}$ post-transfection. Application of $\mathrm{MPP}^{+}$for $24 \mathrm{~h}$ led to a significant cell death of dopaminergic PMNs in all treatment groups. Nevertheless, in miR-182 and miR-183 mimic transfected PMNs as well as in GDNF treated cultures a significantly higher amount of surviving dopaminergic neurons was detected compared to NC siRNA transfected PMNs. Again, the miRNA effects were comparable to the effects of GDNF treatment. As an additional control the dopaminergic survival in miR-1a-3p transfected PMNs was analyzed: there the survival was comparable to NC siRNA transfected PMNs. Consequently, the observed neuroprotective effect of miR-182 and miR-183 transfection is not caused by a mere miRNA increase.

The fact that increased miR-182 and miR-183 levels protect dopaminergic neurons from $\mathrm{MPP}^{+}$induced neurotoxicity is not published so far. $\mathrm{MPP}^{+}$mediates its toxicity via inhibition of complex I of the respiratory chain and leads to increased ROS production and oxidative stress. Interestingly, there are several studies on other cell types that report that miR-182 and miR-183 protect from apoptosis caused by oxidative stress via inhibition of Foxo transcription factors (Gheysarzadeh \& Yazdanparast 2014; Tang et al. 2015). Beside the inhibition of Foxo transcription factors also the up regulation of PI3K-Akt and Mapk signaling and subsequent increase in Bcl-2 expression are known to be involved in the GDNF-mediated protection of dopaminergic neurons from toxin-induced cell death (Sawada et al. 2000; Ugarte et al. 2003). Thus, it is likely that miR-182 and miR-183 induced protection of dopaminergic PMNs is mediated by a decreased Foxo-induced apoptosis and increase in survival signaling.

The precise mechanism how GDNF regulates miR-182 and miR-183 expression and if there is possibly a concerted action or a feedback mechanism between GDNF and the miRNAs is not yet known and requires further investigation. There are reports showing 
that the transcription of the GDNF gene and the miR-96 182 183 cluster are, among others, initiated by the transcription factor Sp1 (He \& Ron 2006; Hung 2013; Yang et al. 2014). It is also known that this transcription factor is involved in GDNF-regulated autoexpression (He \& Ron 2006). Foxo1 reduces the transcriptional capacity of Sp1. Consequently decreased Foxo1 through miR-182 or miR-183 increase might lead to a higher transcriptional activity of Sp1 (Deng et al. 2012), which in turn might lead to an increased neuronal GDNF expression. Another point that favors this hypothesis is the increased activation of the PI3K-Akt and Mapk signaling pathways upon miR-182/miR-183 increase that could easily be induced by secreted GDNF binding to its receptor. Additionally, the activation of PI3K-Akt and Mapk signaling pathways leads to a phosphorylation and thus higher transcriptional activity of Sp1 (Merchant et al. 1999).

In order to prove this hypothetical feedback mechanism between GDNF, miR182/miR-183 and Sp1 (see figure 4.3/1), a series of experiments needs to be performed investigating if increased miR-182 and miR-183 induce GDNF expression via Foxo inhibition and activation of Sp1. Furthermore, experimental evidence is needed to prove that $\mathrm{Sp} 1$ is the transcription factor responsible for GDNF-induced miR-182 and miR-183 expression.

Taken together, the present results show that increased levels of miR-182 and miR-183 mimic GDNF effects with respect to neurite length, regeneration and protection from MPP ${ }^{+}$-induced cell death in dopaminergic PMNs. Furthermore, miR-182 and miR-183 increase as well as GDNF treatment lead to a decreased expression of Foxo transcription factors and an increased activation of PI3K-Akt and Mapk signaling pathways that might mediate the observed effects.

Figure 4.3/1 (following page): Proposed model for GDNF and miR-182/miR-183 interactions and their effects on dopaminergic neurons. (a) Without GDNF treatment or miR-182/miR-183 transfection (Normal state) miR-182/miR-183 are expressed at normal levels, inhibiting Foxo1 and Foxo3 translation. Nuclear Foxo inhibits Sp1 activation, resulting in a limited expression of GDNF and miR-182/miR-183. (b) Binding of GDNF to GDNF family receptor $\alpha-1$ (GFRA1) leads to an activation of PI3K-Akt and Mapk signaling which in turn leads to activation of growth and survival pathways and to nuclear import of Sp1, resulting in increased GDNF and miR-182/miR-183 expression. Increased miR-182/miR-183 levels result in increased translational repression of Foxo and lead subsequently to activation of Sp1. 


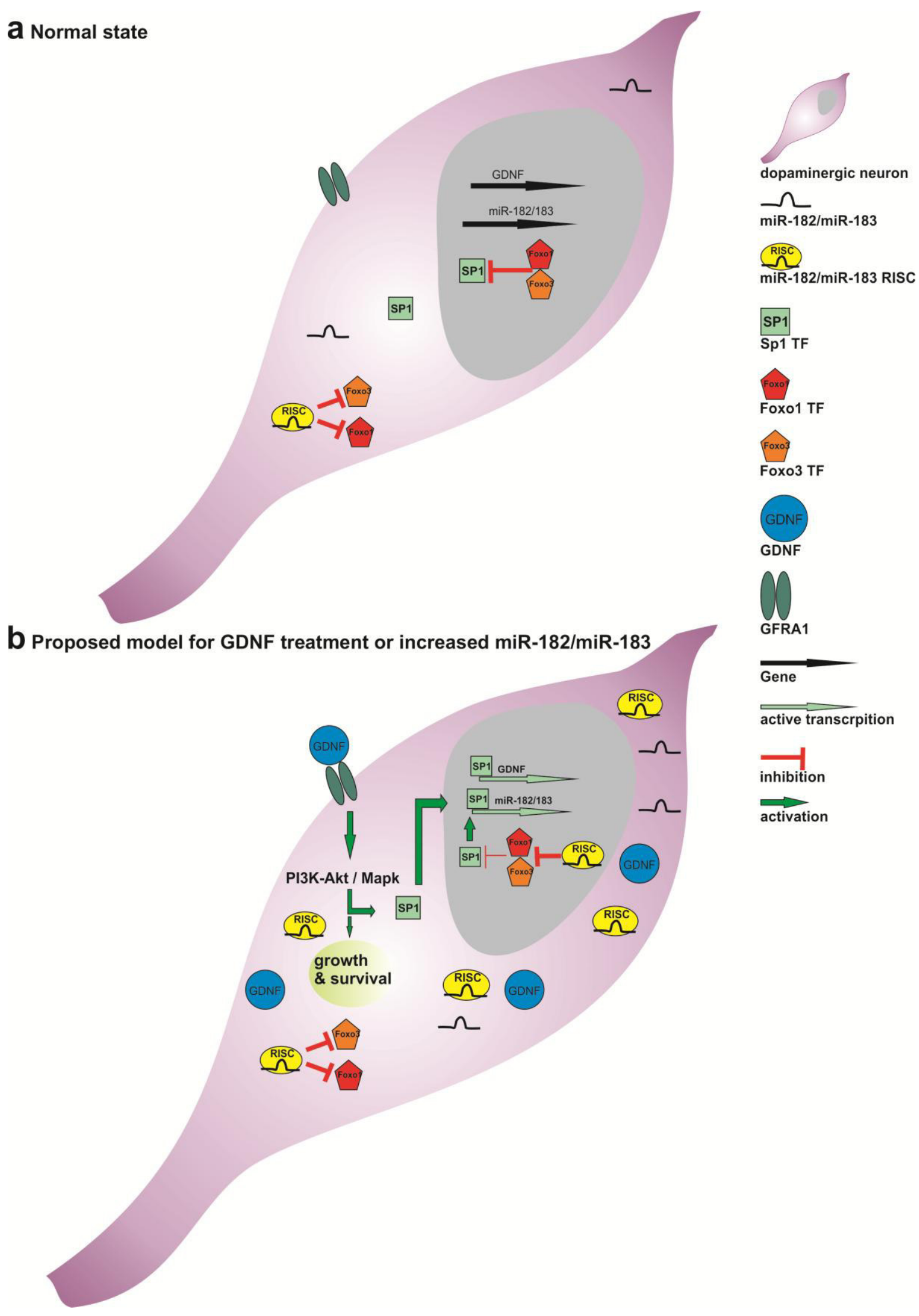




\subsection{Effects of increased miR-182 and miR-183 levels in the MPTP mouse model for PD}

The previous results revealed that increased miR-182 and miR-183 levels lead to an increased neurite growth and regeneration and protect dopaminergic PMNs from $\mathrm{MPP}^{+}$induced cell death, indicating that these miRNAs might be a valuable target for the treatment of PD. Consequently, it was necessary to analyze the effect of increased miR182 and miR-183 in an in vivo setting. In order to test the neuroprotective effect of miR182 and miR-183 in a mouse model for PD the acute MPTP paradigm was applied. The MPTP mouse model has been used for over 20 years and is well characterized (Schober 2004). This model was used in numerous studies for testing of neuroprotective effects of different substances. In the present work the miRNA mimics were injected into the right substantia nigra of mice $48 \mathrm{~h}$ prior to the first MPTP injection to make sure that a sufficient amount of miRNA mimics enters the dopaminergic neurons of the $\mathrm{SN}$ and to have a comparability to the previous in vitro study, where the transfection took place 24 $\mathrm{h}$ before $\mathrm{MPP}^{+}$addition. Additionally, since MPTP-injected animals excrete the toxin and have to be handled with special precautions, stereotactic surgery on MPTP-injected animals is not advised. As miRNAs are highly charged molecules they cannot cross the blood brain barrier and thus have to be injected directly into the brain. The miRNA mimics were mixed with HiPerfect transfection reagent according to a protocol that was already used for hippocampal injection (Banzhaf-Strathmann et al. 2014; Zovoilis et al. 2011). Concentration, injection parameters and coordinates were adapted for injections in the $\mathrm{SN}$ and transfection of dopaminergic neurons in the $\mathrm{SN} 48 \mathrm{~h}$ post-injection was verified by injection of a fluorescently labeled siRNA. Interestingly, the transfected cells were almost exclusively neurons and only very small number of glial cells showed siRNA-mediated fluorescence. This is in accordance with previous studies reporting that the transfection of neurons with small oligonucleotides is very potent and outclasses the transfection of glial cells (Sommer et al. 1998). Quantitative PCR of the SN midbrain region of injected animals demonstrated that the injection of $250 \mathrm{pmol}$ miRNA mimic led to an increase in detected mature miRNAs that was stable even 7 days after injection. 


\subsubsection{Increased levels of miR-182 lead to protection of the nigrostriatal system in MPTP- intoxicated mice and partially rescue behavioral deficits}

Injection of miRNA mimics or NC siRNA in non-intoxicated PBS-injected control animals had no significant effect on TH-positive cell numbers in the ipsilateral SN compared to animals that were systemically treated with PBS but had no stereotactic surgery. Furthermore, the obtained numbers of TH-positive cells in control animals were comparable to previous studies (Saal et al. 2015; Tönges et al. 2012). Thus, the mechanical lesion caused by the nigral injection as well as the injected oligonucleotides have no effect on dopaminergic cell survival in the ipsilateral SNpc. After MPTP intoxication a massive decrease in TH-positive cell numbers of animals injected with NC siRNA was observed and the obtained cell numbers were comparable to previous studies employing the MPTP mouse model (Tönges et al. 2012). Quantification of Nissl positive cells showed a similar decline in neuronal cells, giving evidence that MPTP induced a specific dopaminergic cell death.

Dopaminergic cell survival was significantly higher in animals injected with miR182 mimics and a similar trend was observed in animals that received nigral injections of miR-183 mimics. Compared to the previously performed in vitro study on dopaminergic PMNs, the dopaminergic survival after MPTP-intoxication in vivo is much better than after $\mathrm{MPP}^{+}$intoxication in vitro. A possible reason for this finding is that the brain in a living animal has a higher capacity to cope with toxic insults than isolated neurons in a pure primary cell culture without the support of glial cells. In control PMN cultures 70 percent of the dopaminergic neurons degenerated after $\mathrm{MPP}^{+}$treatment, in comparison the MPTP effect in control mice was milder and led to a degeneration of $\sim 45$ percent of THpositive cells. Interestingly, the rescue effect of miR-182 and miR-183 was similar in MPP ${ }^{+}$ treated PMN cultures, whereas the miR-182 effect exceeded the miR-183 effect in vivo. The protein expression analysis in vitro also revealed a higher effect of miR-182 on the activation of PI3K-Akt and Mapk signaling compared to miR-183, which might reflect the stronger effect of miR-182 in vivo. In the present study an additional GDNF-treated group was not included. Nevertheless, previous studies of other groups using nigral injections of recombinant GDNF in the MPTP mouse model can be utilized for comparison. Injection of recombinant GDNF to the SNpc $24 \mathrm{~h}$ prior to MPTP treatment for 2 consecutive days (40 $\mathrm{mg} / \mathrm{kg} \mathrm{BW}$ ) led to a significant increase in surviving TH-positive neurons in the SNpc. 
Approximately 90 percent of the dopaminergic neurons in the SNpc survived the MPTP intoxication after GDNF treatment (Tomac et al. 1995). Thus, the miR-182 mediated effect on dopaminergic neuron survival ( 92 \% surviving $\mathrm{TH}+$ neurons) is comparable to the effects after GDNF treatment, reflecting the results of the in vitro studies on PMNs. The results from the neuronal PMN cultures showing a specific effect of GDNF on dopaminergic neurons leading to an upregulation of the analyzed miRNAs indicate that miR-182 and miR-183 act on dopaminergic neurons. This is further supported by the preferential transfection of neurons in the brain (Sommer et al. 1998). Of course it cannot be excluded that other neuronal cells in the SNpc mediate the neuroprotective effect of miR-182/miR-183 on dopaminergic cells as a secondary effect, but as the majority of neurons in the SNpc are dopaminergic this is not very likely. The results of the PMN study revealed that the down-regulation of Foxo transcription factors and an increased activity of pathways involved in survival and growth after miR-182 and miR-183 transfection are involved in mediation of the miRNA effects.

The histological analysis of DAT-positive striatal fibers revealed a more pronounced degeneration of striatal dopaminergic terminals than cell bodies in the SNpc. This reflects the situation in PD patients where with onset of motor symptoms already $\sim 70$ to 80 percent of the nigrostriatal injections are degenerated but only $\sim 30$ percent of nigral dopaminergic neurons are missing (Burke \& O'Malley 2013). This suggests that the degenerative process in PD starts at the nerve terminals and that a so called 'die back' pathology leads to loss of nigral neurons (Dauer \& Przedborski 2003). This phenomenon is also occurring in the MPTP mouse model for PD, explaining the larger impact on the striatal terminals (Li et al. 2009; Tomac et al. 1995). Nevertheless, nigral miR-182 mimic injection led to a significant decrease in dopaminergic fiber degeneration in the striatum. A similar trend was observed in miR-183-treated animals, although this was not significant. The extent of the observed effects is comparable to findings in GDNF-treated animals (Tomac et al. 1995), indicating again that miR-182/miR-183 are involved in GDNFmediated protection of dopaminergic neurons. In order to determine whether the observed morphological preservation of dopaminergic striatal fibers correlates with functional maintenance of these axonal nerve terminals, the content of dopamine and its metabolites DOPAC and HVA in the striatum were measured by HPLC. The extent of DA, DOPAC and HVA depletion was more severe than the reduction of striatal fibers. 
Nevertheless, miR-182-injected animals showed a significant higher amount of striatal DA compared to NC siRNA-injected animals. In miR-183-injected mice there was no increase in striatal DA observable. The amount of DA metabolites was not significantly changed in the miRNA-injected animals compared to NC siRNA-injected animals. Thus, there is functional preservation of dopaminergic terminals in miR-182 injected animals but the morphological preservation is much more pronounced. Interestingly, again the present data is comparable to the results of the study analyzing the effect of nigral GDNF injection in the MPTP mouse model. There, the depletion of DA and its metabolites in the striatum was also more extensive than the morphological degeneration and GDNF application led only to a small but significant increase in striatal DA (Tomac et al. 1995). Taken together, nigral injections of miR-182 (and to a lesser extent of miR-183) led to a preservation of dopaminergic neurons in the SNpc and their nigrostriatal projections in MPTP-intoxicated animals that is comparable to the effect of nigral GDNF application. Thereby, the morphological preservation was more pronounced than the functional preservation similar to previous studies investigating the neuroprotective effects of different substances (Saal et al. 2015; Tomac et al. 1995; Tönges et al. 2012).

The neuroprotective effects of miR-182 and miR-183 injections could also be visualized in a behavioral analysis of motor functions utilizing the cylinder test. The cylinder test gives information about whole body coordination and forelimb use. A bias towards a higher number of rears assisted with both paws observed in NC siRNA treated and MPTP-intoxicated animals was rescued in miR-182 and miR-183 injected MPTPintoxicated mice, showing no significant difference to PBS treated control animals. Keeping in mind the mild increase in striatal DA in miR-182 injected animals, the observed clear behavioral response is somewhat surprising. This could either indicate that the behavioral paradigm was not sensitive enough, even though it was demonstrated in other studies that it is adequate for investigation of motor behavior in the MPTP mouse model (Tönges et al. 2012), or that the moderate increase in striatal DA was sufficient to restore motor behavior in this task. Interestingly, the study by Tomac et al. (1995), investigating the effect of nigral injections of recombinant GDNF on the MPTP mouse model, shows similar findings. As mentioned above, GDNF application led to a morphological preservation of dopaminergic neurons and striatal terminals and to a mild increase in striatal DA after MPTP intoxication compared to control mice and this was accompanied 
by a strong rescue effect on the rearing behavior. Additionally, the present results show that the cylinder test is sensitive enough to detect motor impairment after MPTP treatment, indicating that the test is sufficient for testing motor behavior after MPTP treatment. Considering the discrepancy between onset of motor symptoms and loss of nigrostriatal projections in PD it would be reasonable that a slight improvement of striatal DA content could lead to pronounced behavioral effects. Furthermore, in comparison with humans, rodents with a damaged nigrostriatal sytem show very pronounced compensatory mechanisms that rescue behavioral deficits.

Taken together, the present study gives evidence that increased levels of miR-182 and miR-183 in the SN lead to a rescue of MPTP-mediated behavioral deficits.

\section{5 miRNAs as therapeutic targets}

Since their discovery miRNAs have been extensively studied and there is a growing body of evidence that they might be viable therapeutic targets for different diseases, including neurodegenerative diseases, cancer and cardiovascular diseases. As a single miRNA modulates the expression of a high number of different genes, they simultaneously control multiple cellular pathways. This entails different risks and chances. Of course, it would be valuable to replace or inhibit a miRNA that is deregulated. On the other hand, several safety concerns are important to consider. In order to avoid off-target side effects it would be important to restrict the replacement/inhibition to the cell type and organ of interest and to clearly understand the pharmacokinetics of miRNA (Bouchie 2013). Thus, the application mode of miRNA-based therapeutics is crucial. There are different possibilities to replace or inhibit miRNAs, such as expression of miRNA or miRNA inhibitor constructs by viral vectors as well as application of synthetic miRNA mimics or antisense oligonucleotides (inhibitors).

Viral vectors expressing shRNA- or miRNA-constructs lead to a constant expression of their construct. The possibility to use different serotypes in combination with tissue specific promoters allows a relatively precise transduction of the cell type/tissue of interest (van Rooij et al. 2012). However, there is a growing body of evidence that AAVmediated shRNA/miRNA overexpression has toxic side effects, presumably due to a saturation of the endogenous miRNA machinery (Ehlert et al. 2010; Grimm 2011). Thus, 
the development and application of synthetic miRNA mimics or inhibitors has become more important. Several modifications of synthetic oligonucleotides have been employed to improve their cellular uptake and affinity. The major challenges in synthetic miRNA therapeutics are a targeted delivery and prevention of introduction of supraphysiological levels (E. van Rooij \& Kauppinen 2014). Possible strategies are conjugation of the nucleic acid with targeting molecules or encapsulation to enhance cell specific uptake. Until a more specific application is possible, local device based application by catheters or local injection could be applied, which of course implies the risk of all invasive procedures, such as bleeding and infection. So far many miRNA based therapeutics were tested in preclinical studies but only few entered clinical trials. Nevertheless, clinical phase I and II trials with miRNA mimics (miR-34; liver cancer (Bouchie 2013)) or inhibitors (miR-122; Hepatitis C (Janssen et al. 2013)) demonstrated their promising therapeutic potential and implied that miRNA-based therapeutics can indeed become reality. 


\section{Concluding remarks}

All experiments performed in the context of this thesis underline the importance of miRNAs in dopaminergic neurons and the nigrostriatal system. The present study gives evidence that a pronounced regulation of miRNA expression is involved in the maturation of PMNs. Additionally, two novel GDNF-regulated miRNAs were detected and their effect on dopaminergic neurons in vitro and in vivo was extensively studied. Furthermore, a novel set of miRNAs involved in the degeneration and spontaneous recovery of dopaminergic neurons was presented.

The investigation of GDNF-regulated miR-182 and miR-183 revealed that higher levels of these miRNAs lead to increased neurite outgrowth and regeneration in dopaminergic PMNs and protect those cells from $\mathrm{MPP}^{+}$toxicity. These effects were mediated by the down-regulation of Foxo transcription factors and increased activation of PI3K-Akt and Mapk signaling pathways. Interestingly, the miRNA effects were in most cases comparable to GDNF-mediated effects, leading to the assumption that GDNF acts at least partially via miR-182 and miR-183. In the MPTP mouse model for PD nigral injection of miR-182 mimics led to morphological and, to a lesser extent, functional preservation of dopaminergic neurons and their nigrostriatal projections. Furthermore, the MPTPmediated behavioral deficits were restored by miR-182 or miR-183 mimic injections in the SN. Further experiments are needed to completely understand the interplay between GDNF and miR-182/miR-183.

The present work underscores that miRNAs are important regulators in dopaminergic neuron development and function in health and disease and that they have the potential to act neuroprotectively or induce neuroregeneration in models for PD. Thus, miRNAs are auspicious targets to find new therapeutic strategies for the treatment of PD and a valuable tool to obtain a better understanding of the underlying disease mechanisms. 


\section{Summary}

$\mathrm{PD}$ is the second most frequent neurodegenerative disorder affecting over $3 \%$ of the population older than 80 years of age. Although PD is a system disorder which affects most regions of the brain, the progressive demise of the nigrostriatal projections and the inability of this system to regenerate are mainly responsible for the functional deficits observed. The etiology underlying PD is not finally resolved. Recent studies suggest that gene expression regulators might contribute to a large variety of disease states. miRNAs are small non-coding RNAs that are important for the post-transcriptional regulation of gene expression. Alterations in miRNA function have been reported in different neurodegenerative diseases including PD. Furthermore, miRNAs are auspicious therapeutic targets, as the manipulation of their expression might be neuroprotective or could induce the regeneration of neurons.

In the present study the miRNAome of developing PMN cultures was analyzed and it was demonstrated that the major changes occur during early development. Furthermore, the influence of GDNF on the PMN miRNAome was investigated and a specific up-regulation of miR-182 and miR-183 upon GDNF treatment was discovered. In order to analyze miRNA expression changes during degeneration and spontaneous regeneration of the murine nigrostriatal system a small RNA sequencing of the midbrain of the 6-OHDA mouse model for PD was performed and revealed novel sets of miRNAs involved in degeneration and regeneration of DA neurons in vivo.

With the objective to investigate the effect of increased GDNF-regulated miR-182 and miR-183 levels on DA neurons, transfection of PMNs with miRNA mimics was performed and revealed increased neurite growth and regeneration, as well as protection of DA PMNs from MPP ${ }^{+}$toxicity. Increased miR-182 and miR-183 led to decreased levels of Foxo transcription factors and increased PI3K-Akt and MAPK signaling, that presumably mediate the reported effects. Application of miR-182/miR-183 mimics in vivo revealed morphological and partially functional preservation of DA neurons, as well as restoration of rearing behavior after MPTP treatment.

Taken together, miRNAs play an important role in development, maintenance and degeneration of DA neurons in vivo and in vitro and thus are promising targets for a better understanding of PD pathophysiology and the development of new therapeutic strategies. 


\section{References}

Alexandrov, P. N., Dua, P., Hill, J. M., Bhattacharjee, S., Zhao, Y., \& Lukiw, W. J. (2012). microRNA (miRNA) speciation in Alzheimer's disease (AD) cerebrospinal fluid (CSF) and extracellular fluid (ECF). International Journal of Biochemistry and Molecular Biology, 3(4), 365-73.

Alvarez-Fischer, D., Henze, C., Strenzke, C., Westrich, J., Ferger, B., Höglinger, G. U., ... Hartmann, A. (2008). Characterization of the striatal 6-OHDA model of Parkinson's disease in wild type and $\alpha$-synuclein-deleted mice. Experimental Neurology.

Anitha, M., Gondha, C., Sutliff, R., Parsadanian, A., Mwangi, S., Sitaraman, S. V, \& Srinivasan, S. (2006). GDNF rescues hyperglycemia-induced diabetic enteric neuropathy through activation of the PI3K/Akt pathway. The Journal of Clinical Investigation, 116(2), 344-56.

Arasu, P., Wightman, B., \& Ruvkun, G. (1991). Temporal regulation of lin-14 by the antagonistic action of two other heterochronic genes, lin-4 and lin-28. Genes \& Development, 5(10), 1825-1833.

Bankiewicz, K. S., Plunkett, R. J., Jacobowitz, D. M., Porrino, L., di Porzio, U., London, W. T., ... Oldfield, E. H. (1990). The effect of fetal mesencephalon implants on primate MPTP-induced parkinsonism. Histochemical and behavioral studies. Journal of Neurosurgery, 72(2), 231-44.

Banzhaf-Strathmann, J., Benito, E., May, S., Arzberger, T., Tahirovic, S., Kretzschmar, H., ... Edbauer, D. (2014). MicroRNA-125b induces tau hyperphosphorylation and cognitive deficits in Alzheimer's disease. The EMBO Journal, 33(15), 1667-80.

Benabid, A. L., Chabardes, S., Mitrofanis, J., \& Pollak, P. (2009). Deep brain stimulation of the subthalamic nucleus for the treatment of Parkinson's disease. The Lancet. Neurology, 8(1), 67-81.

Beveridge, N. J., Tooney, P. A., Carroll, A. P., Tran, N., \& Cairns, M. J. (2009). Downregulation of miR-17 family expression in response to retinoic acid induced neuronal differentiation. Cellular Signalling, 21(12), 1837-45.

Bezard, E., \& Przedborski, S. (2011). A tale on animal models of Parkinson's Disease. Movement Disorders, 26(6), 993-1002.

Bhomia, M., Balakathiresan, N., Sharma, A., Gupta, P., Biswas, R., \& Maheshwari, R. (2010). Analysis of microRNAs induced by Venezuelan equine encephalitis virus infection in mouse brain. Biochemical and Biophysical Research Communications, 395(1), 11-16. 
Bian, S., Hong, J., Li, Q., Schebelle, L., Pollock, A., Knauss, J. L., ... Sun, T. (2013). MicroRNA Cluster miR-17-92 Regulates Neural Stem Cell Expansion and Transition to Intermediate Progenitors in the Developing Mouse Neocortex. Cell Reports, 3(5), 1398-1406.

Bjorklund, L. M., Sánchez-Pernaute, R., Chung, S., Andersson, T., Chen, I. Y. C., McNaught, K. S. P., ... Isacson, O. (2002). Embryonic stem cells develop into functional dopaminergic neurons after transplantation in a Parkinson rat model. Proceedings of the National Academy of Sciences of the United States of America, 99(4), 2344-9.

Bonni, A., Brunet, A., West, a E., Datta, S. R., Takasu, M. a, \& Greenberg, M. E. (1999). Cell survival promoted by the Ras-MAPK signaling pathway by transcription-dependent and -independent mechanisms. Science (New York, N.Y.), 286, 1358-1362.

Borek, L. L., Kohn, R., \& Friedman, J. H. (2007). Phenomenology of dreams in Parkinson's disease. Movement Disorders, 22(2), 198-202.

Bouchie, A. (2013). First microRNA mimic enters clinic. Nature Biotechnology, 31(7), 577577.

Braak, H., Rüb, U., Gai, W. P., \& Del Tredici, K. (2003). Idiopathic Parkinson's disease: Possible routes by which vulnerable neuronal types may be subject to neuroinvasion by an unknown pathogen. Journal of Neural Transmission, 110(5), 517-536.

Braak, H., Tredici, K. Del, Rüb, U., de Vos, R. A. ., Jansen Steur, E. N. ., \& Braak, E. (2003). Staging of brain pathology related to sporadic Parkinson's disease. Neurobiology of Aging, 24(2), 197-211.

Bredesen, D. E., Rao, R. V., \& Mehlen, P. (2006). Cell death in the nervous system. Nature, 443(7113), 796-802.

Briggs, C. E., Wang, Y., Kong, B., Woo, T.-U. W., lyer, L. K., \& Sonntag, K. C. (2015). Midbrain dopamine neurons in Parkinson's disease exhibit a dysregulated miRNA and target-gene network. Brain Research, 1618, 111-21.

Bronstein, J. M., Tagliati, M., Alterman, R. L., Lozano, A. M., Volkmann, J., Stefani, A., ... DeLong, M. R. (2011). Deep Brain Stimulation for Parkinson Disease. Archives of Neurology, 68(2), 165-171.

Brunet, a, Datta, S. R., \& Greenberg, M. E. (2001). Transcription-dependent and independent control of neuronal survival by the PI3K-Akt signaling pathway. Curr.Opin.Neurobiol., 11(3), 297-305.

Brunet, A., Datta, S. R., \& Greenberg, M. E. (2001). Transcription-dependent and independent control of neuronal survival by the PI3K-Akt signaling pathway. Current Opinion in Neurobiology, 11(3), 297-305. 
Burke, R. E. (2007). Inhibition of mitogen-activated protein kinase and stimulation of Akt kinase signaling pathways: Two approaches with therapeutic potential in the treatment of neurodegenerative disease. Pharmacology \& Therapeutics, 114(3), 261-77.

Burke, R. E., \& O'Malley, K. (2013). Axon degeneration in Parkinson's disease. Experimental Neurology, 246, 72-83.

Cao, J., Shen, Y., Zhu, L., Xu, Y., Zhou, Y., Wu, Z., ... Zhu, X. (2012). miR-129-3p controls cilia assembly by regulating CP110 and actin dynamics. Nature Cell Biology, 14(7), 697706.

Carnicella, S., Kharazia, V., Jeanblanc, J., Janak, P. H., \& Ron, D. (2008). GDNF is a fastacting potent inhibitor of alcohol consumption and relapse. Proceedings of the National Academy of Sciences of the United States of America, 105(23), 8114-9.

Castanotto, D., \& Rossi, J. J. (2009). The promises and pitfalls of RNA-interference-based therapeutics. Nature, 457(7228), 426-33.

Cheng, F.-C., Ni, D.-R., Wu, M.-C., \& Kuo, J.-S. (1998). Glial cell line-derived neurotrophic factor protects against 1-methyl-4-phenyl-1,2,3,6-tetrahydropyridine (MPTP)induced neurotoxicity in C57BL/6 mice. Neuroscience Letters, 252(2), 87-90.

Chiang, H. R., Schoenfeld, L. W., Ruby, J. G., Auyeung, V. C., Spies, N., Baek, D., ... Bartel, D. P. (2010). Mammalian microRNAs: Experimental evaluation of novel and previously annotated genes. Genes and Development, 24(10), 992-1009.

Cho, Y., \& Cavalli, V. (2014). HDAC signaling in neuronal development and axon regeneration. Current Opinion in Neurobiology, 27, 118-126.

Chong, Z. Z., Li, F., \& Maiese, K. (2008). Group I Metabotropic Receptor Neuroprotection Requires Akt and Its Substrates that Govern FOXO3a, Bim, and $\beta$-Catenin During Oxidative Stress. Curr Neurovasc Res., 15(10), 1203-1214. http://doi.org/10.1016/j.drugalcdep.2008.02.002.A

Cogswell, J. P., Ward, J., Taylor, I. a, Waters, M., Shi, Y., Cannon, B., ... Richards, C. a. (2008). Identification of miRNA changes in Alzheimer's disease brain and CSF yields putative biomarkers and insights into disease pathways. Journal of Alzheimer's Disease : JAD, 14(1), 27-41.

Connolly, B. S., \& Lang, A. E. (2014). Pharmacological Treatment of Parkinson Disease. Jama, 311(16), 1670.

Czabotar, P. E., Lessene, G., Strasser, A., \& Adams, J. M. (2013). Control of apoptosis by the BCL-2 protein family: implications for physiology and therapy. Nature Reviews Molecular Cell Biology, 15(1), 49-63. 
Dauer, W., \& Przedborski, S. (2003). Parkinson's Disease: Mechanisms and Models. Neuron, 39, 889-909.

Davis, T. H., Cuellar, T. L., Koch, S. M., Barker, A. J., Harfe, B. D., McManus, M. T., \& Ullian, E. M. (2008). Conditional loss of Dicer disrupts cellular and tissue morphogenesis in the cortex and hippocampus. The Journal of Neuroscience : The Official Journal of the Society for Neuroscience, 28(17), 4322-30.

Dawson, T. M., \& Dawson, V. L. (2002). Neuroprotective and neurorestorative strategies for Parkinson's disease. Nature Neuroscience, 5(Supp), 1058-1061.

de Lau, L. M. L., \& Breteler, M. M. B. (2006). Epidemiology of Parkinson's disease. The Lancet. Neurology, 5(6), 525-35.

De Pietri Tonelli, D., Pulvers, J. N., Haffner, C., Murchison, E. P., Hannon, G. J., \& Huttner, W. B. (2008). miRNAs are essential for survival and differentiation of newborn neurons but not for expansion of neural progenitors during early neurogenesis in the mouse embryonic neocortex. Development (Cambridge, England), 135(23), 3911-21.

Deng, X., Dzamko, N., Prescott, A., Davies, P., Liu, Q., Yang, Q., ... Gray, N. S. (2011). Characterization of a selective inhibitor of the Parkinson's disease kinase LRRK2. Nature Chemical Biology, 7(4), 203-5.

Deng, X., Zhang, W., O-Sullivan, I., Williams, J. B., Dong, Q., Park, E. A., ... Elam, M. B. (2012). FoxO1 inhibits sterol regulatory element-binding protein-1c (SREBP-1c) gene expression via transcription factors Sp1 and SREBP-1c. The Journal of Biological Chemistry, 287(24), 20132-43.

Dexter, D. T., \& Jenner, P. (2013). Parkinson disease: from pathology to molecular disease mechanisms. Free Radical Biology \& Medicine, 62, 132-44.

Doxakis, E. (2010). Post-transcriptional regulation of alpha-synuclein expression by mir-7 and mir-153. The Journal of Biological Chemistry, 285(17), 12726-34.

Edbauer, D., Neilson, J. R., Foster, K. A., Wang, C.-F., Seeburg, D. P., Batterton, M. N., ... Sheng, M. (2010). Regulation of synaptic structure and function by FMRP-associated microRNAs miR-125b and miR-132. Neuron, 65(3), 373-84.

Eddy, S. R. (2001). Non-coding RNA genes and the modern RNA world. Nature Reviews. Genetics, 2(12), 919-29.

Ehlert, E. M., Eggers, R., Niclou, S. P., \& Verhaagen, J. (2010). Cellular toxicity following application of adeno-associated viral vector-mediated RNA interference in the nervous system. BMC Neuroscience, 11(1), 20.

Elsworth, J. D., Taylor, J. R., Sladek, J. R., Collier, T. J., Redmond, D. E., \& Roth, R. H. (1999). Striatal dopaminergic correlates of stable parkinsonism and degree of recovery in 
old-world primates one year after MPTP treatment. Neuroscience, 95(2), 399-408.

Exner, N., Lutz, A. K., Haass, C., \& Winklhofer, K. F. (2012). Mitochondrial dysfunction in Parkinson's disease: molecular mechanisms and pathophysiological consequences. The EMBO Journal, 31(14), 3038-3062.

Fabian, M., Sonenberg, N., \& Filipowicz, W. (2010). Regulation of mRNA translation and stability by microRNAs. Annu Rev Biochem., 79, 351-379.

Finkelstein, D. I., Stanic*, D., Parish, C. L., Tomas, D., Dickson, K., \& Horne, M. K. (2000). Axonal sprouting following lesions of the rat substantia nigra. Neuroscience, 97(1), 99-112. http://doi.org/10.1016/S0306-4522(00)00009-9

Fitch, M. T., \& Silver, J. (2008). CNS injury, glial scars, and inflammation: Inhibitory extracellular matrices and regeneration failure. Experimental Neurology, 209(2), 294-301.

Forman, M. S., Trojanowski, J. Q., \& Lee, V. M. (2004). Neurodegenerative diseases: a decade of discoveries paves the way for therapeutic breakthroughs. Nature Medicine, 10(10), 1055-1063.

Freed, C. R., Greene, P. E., Breeze, R. E., Tsai, W.-Y., DuMouchel, W., Kao, R., ... Fahn, S. (2001). Transplantation of Embryonic Dopamine Neurons for Severe Parkinson's Disease. The New England Journal of Medicine, 344(10), 710-719.

Gao, F.-B. (2010). Context-dependent functions of specific microRNAs in neuronal development. Neural Development, 5(1), 25.

Gaughwin, P. M., Ciesla, M., Lahiri, N., Tabrizi, S. J., Brundin, P., \& Björkqvist, M. (2011). Hsa-miR-34b is a plasma-stable microRNA that is elevated in pre-manifest Huntington's disease. Human Molecular Genetics, 20(11), 2225-37.

Gehrke, S., Imai, Y., Sokol, N., \& Lu, B. (2010). Pathogenic LRRK2 negatively regulates microRNA-mediated translational repression. Nature, 466(7306), 637-641.

Gerlach, M., Riederer, P., Przuntek, H., \& Youdim, M. B. H. (1991). MPTP mechanisms of neurotoxicity and their implications for Parkinson's disease. European Journal of Pharmacology: Molecular Pharmacology, 208(4), 273-286.

Gheysarzadeh, A., \& Yazdanparast, R. (2014). STAT5 Reactivation by Catechin Modulates H2O2-Induced Apoptosis Through miR-182/FOXO1 Pathway in SK-N-MC Cells. Cell Biochemistry and Biophysics, 71(2), 649-656.

Ghildiyal, M., \& Zamore, P. D. (2009). Small silencing RNAs: an expanding universe. Nature Reviews. Genetics, 10(2), 94-108.

Giasson, B. I., \& Lee, V. M.-Y. (2003). Are Ubiquitination Pathways Central to Parkinson's Disease? Cell, 114(1), 1-8. 
Grimm, D. (2011). The dose can make the poison: lessons learned from adverse in vivo toxicities caused by RNAi overexpression. Silence, $2(1), 8$.

Gu, T., Zhao, T., \& Hewes, R. S. (2014). Insulin signaling regulates neurite growth during metamorphic neuronal remodeling. Biology Open, 3(1), 81-93.

Guttilla, I. K., \& White, B. A. (2009). Coordinate Regulation of FOXO1 by miR-27a, miR-96, and miR-182 in Breast Cancer Cells. Journal of Biological Chemistry, 284(35), 2320423216.

Hanisch, U.-K., \& Kettenmann, H. (2007). Microglia: active sensor and versatile effector cells in the normal and pathologic brain. Nature Neuroscience, 10(11), 1387-1394.

Harraz, M. M., Eacker, S. M., Wang, X., Dawson, T. M., \& Dawson, V. L. (2012). MicroRNA223 is neuroprotective by targeting glutamate receptors. Proceedings of the National Academy of Sciences of the United States of America, 109(46), 18962-7.

He, D.-Y., \& Ron, D. (2006). Autoregulation of glial cell line-derived neurotrophic factor expression: implications for the long-lasting actions of the anti-addiction drug, Ibogaine. FASEB Journal : Official Publication of the Federation of American Societies for Experimental Biology, 20(13), 2420-2.

Hébert, S. S., Horré, K., Nicolaï, L., Papadopoulou, A. S., Mandemakers, W., Silahtaroglu, A. N., ... De Strooper, B. (2008). Loss of microRNA cluster miR-29a/b-1 in sporadic Alzheimer's disease correlates with increased BACE1/beta-secretase expression. Proceedings of the National Academy of Sciences of the United States of America, 105(17), 6415-20.

Henchcliffe, C., \& Beal, M. F. (2008). Mitochondrial biology and oxidative stress in Parkinson disease pathogenesis. Nature Clinical Practice Neurology, 4(11), 600-609.

Heyer, M. P., Pani, A. K., Smeyne, R. J., Kenny, P. J., \& Feng, G. (2012). Normal midbrain dopaminergic neuron development and function in miR-133b mutant mice. The Journal of Neuroscience: The Official Journal of the Society for Neuroscience, 32(32), 10887-94.

Hirsch, E. C., \& Hunot, S. (2009). Neuroinflammation in Parkinson's disease: a target for neuroprotection? The Lancet. Neurology, 8(4), 382-97.

Höglinger, G. U., Rizk, P., Muriel, M. P., Duyckaerts, C., Oertel, W. H., Caille, I., \& Hirsch, E. C. (2004). Dopamine depletion impairs precursor cell proliferation in Parkinson disease. Nature Neuroscience, 7(7), 726-735.

Hou, J.-G. G., Lin, L.-F. H., \& Mytilineou, C. (1996). Glial Cell Line-Derived Neurotrophic Factor Exerts Neurotrophic Effects on Dopaminergic Neurons In Vitro and Promotes Their Survival and Regrowth After Damage by 1-Methyl-4-Phenylpyridinium. Journal 
of Neurochemistry, 66(1), 74-82.

Hsu, S. Da, Tseng, Y. T., Shrestha, S., Lin, Y. L., Khaleel, A., Chou, C. H., ... Huang, H. Da. (2014). MiRTarBase update 2014: An information resource for experimentally validated miRNA-target interactions. Nucleic Acids Research, 42(D1), 78-85.

Huang, D. W., Lempicki, R. A., \& Sherman, B. T. (2009). Systematic and integrative analysis of large gene lists using DAVID bioinformatics resources. Nature Protocols, 4(1), 4457.

Huang, T., Liu, Y., Huang, M., Zhao, X., \& Cheng, L. (2010). Wnt1-cre-mediated conditional loss of Dicer results in malformation of the midbrain and cerebellum and failure of neural crest and dopaminergic differentiation in mice. Journal of Molecular Cell Biology, 2(3), 152-63.

Hung, J.-J. (2013). Enhancement of miR-182 expression by Sp1 promotes lung cancer cell progression. FASEB J, 27(1_MeetingAbstracts), 550.13.

Hurtig, H. I. (1997). Problems with current pharmacologic treatment of Parkinson's disease. Experimental Neurology, 144(1), 10-6.

Hwang, D., Ardayfio, P., Kang, U., Semina, E., \& Kim, K.-S. (2003). Selective loss of dopaminergic neurons in the substantia nigra of Pitx3-deficient aphakia mice. Molecular Brain Research, 114(2), 123-131.

Im, H.-I., Hollander, J. A., Bali, P., \& Kenny, P. J. (2010). MeCP2 controls BDNF expression and cocaine intake through homeostatic interactions with microRNA-212. Nature Neuroscience, 13(9), 1120-7.

Impey, S., Obrietan, K., \& Storm, D. R. (1999). Making new connections: Role of ERK/MAP kinase signaling in neuronal plasticity. Neuron, 23(1), 11-14.

Janetzky, B., Hauck, S., Youdim, M. B. H., Riederer, P., Jellinger, K., Pantucek, F., ... Reichmann, H. (1994). Unaltered aconitase activity, but decreased complex I activity in substantia nigra pars compacta of patients with Parkinson's disease. Neuroscience Letters, 169(1-2), 126-128.

Jankovic, J. (2008). Parkinson's disease: clinical features and diagnosis. Journal of Neurology, Neurosurgery \& Psychiatry, 79(4), 368-376.

Janssen, H. L. A., Reesink, H. W., Lawitz, E. J., Zeuzum, S., Rodriguez-Torres, M., Patel, K., ... Hodges, M. R. (2013). Treatment of HCV Infection by Targeting MicroRNA. The New England Journal of Medicine, (386), 1685-1694.

Jee, M. K., Jung, J. S., Choi, J. I., Jang, J. A., Kang, K. S., Im, Y. Bin, \& Kang, S. K. (2012). MicroRNA 486 is a potentially novel target for the treatment of spinal cord injury. Brain : A Journal of Neurology, 135(Pt 4), 1237-52. 
Jegga, A. G., Schneider, L., Ouyang, X., \& Zhang, J. (2011). Systems biology of the autophagy-lysosomal pathway. Autophagy, 7(5), 477-489.

Jellinger, K. a. (2012). Neuropathology of sporadic Parkinson's disease: Evaluation and changes of concepts. Movement Disorders, 27(1), 8-30.

Jeon, Y.-J., Kim, I. K., Hong, S.-H., Nan, H., Kim, H.-J., Lee, H.-J., ... Jung, Y.-K. (2008). Ribosomal protein $\mathrm{S} 6$ is a selective mediator of TRAIL-apoptotic signaling. Oncogene, $27(31), 4344-4352$.

Jimenez-Mateos, E. M., Engel, T., Merino-Serrais, P., McKiernan, R. C., Tanaka, K., Mouri, G., ... Henshall, D. C. (2012). Silencing microRNA-134 produces neuroprotective and prolonged seizure-suppressive effects. Nature Medicine, 18(7), 1087-94.

Johnson, R., Zuccato, C., Belyaev, N. D., Guest, D. J., Cattaneo, E., \& Buckley, N. J. (2008). A microRNA-based gene dysregulation pathway in Huntington's disease. Neurobiology of Disease, 29(3), 438-45.

Jomova, K., Vondrakova, D., Lawson, M., \& Valko, M. (2010). Metals, oxidative stress and neurodegenerative disorders. Molecular and Cellular Biochemistry, 345(1-2), 91-104.

Jovicic, A., Zaldivar Jolissaint, J. F., Moser, R., Silva Santos, M. de F., \& Luthi-Carter, R. (2013). MicroRNA-22 (miR-22) overexpression is neuroprotective via general antiapoptotic effects and may also target specific Huntington's disease-related mechanisms. PloS One, 8(1), e54222.

Junn, E., Lee, K.-W., Jeong, B. S., Chan, T. W., Im, J.-Y., \& Mouradian, M. M. (2009). Repression of alpha-synuclein expression and toxicity by microRNA-7. Proceedings of the National Academy of Sciences of the United States of America, 106(31), 13052-7.

Kapsimali, M., Kloosterman, W. P., de Bruijn, E., Rosa, F., Plasterk, R. H. A., \& Wilson, S. W. (2007). MicroRNAs show a wide diversity of expression profiles in the developing and mature central nervous system. Genome Biology, 8(8), R173.

Kearns, C. M., \& Gash, D. M. (1995). GDNF protects nigral dopamine neurons against 6hydroxydopamine in vivo. Brain Research, 672(1-2), 104-111.

Kennedy, S. G., Wagner, A. J., Conzen, S. D., Jordán, J., Bellacosa, A., Tsichlis, P. N., \& Hay, N. (1997). The PI 3-kinase/Akt signaling pathway delivers an anti-apoptotic signal. Genes and Development, 11(6), 701-713.

Kettenmann, H., Hanisch, U., Noda, M., \& Verkhratsky, A. (2011). Physiology of Microglia, 461-553.

Kim, D., Nguyen, M. D., Dobbin, M. M., Fischer, A., Sananbenesi, F., Rodgers, J. T., ... Tsai, L.-H. (2007). SIRT1 deacetylase protects against neurodegeneration in models for Alzheimer's disease and amyotrophic lateral sclerosis. The EMBO Journal, 26(13), 
3169-79.

Kim, J., Inoue, K., Ishii, J., Vanti, W. B., Voronov, S. V, Murchison, E., ... Abeliovich, A. (2007). A MicroRNA feedback circuit in midbrain dopamine neurons. Science (New York, N.Y.), 317(5842), 1220-4. http://doi.org/10.1126/science.1140481

Kim, Y., \& Kim, V. N. (2012). MicroRNA factory: RISC assembly from precursor microRNAs. Molecular Cell, 46(4), 384-6.

Kitada, T., Asakawa, S., Hattori, N., Matsumine, H., Yamamura, Y., Minoshima, S., ... Shimizu, N. (1998). Mutations in the parkin gene cause autosomal recessive juvenile parkinsonism. Nature, 392(6676), 605-8.

Klein, M. E., Lioy, D. T., Ma, L., Impey, S., Mandel, G., \& Goodman, R. H. (2007). Homeostatic regulation of MeCP2 expression by a CREB-induced microRNA. Nature Neuroscience, 10(12), 1513-4.

Kopin, I. J. (1992). Features of the dopaminergic neurotoxin MPTP. Annals of the New York Academy of Sciences.

Kordower, J. H., Emborg, M. E., Bloch, J., Shuang, Y. M., Chu, Y., Leventhal, L., ... Aebischer, P. (2000). Neurodegeneration Prevented by Lentiviral Vector Delivery of GDNF in Primate Models of Parkinson's Disease. Science, 290, 767-773.

Krieglstein, K., Suter-Crazzolara, C., Fischer, W. H., \& Unsicker, K. (1995). TGF-beta superfamily members promote survival of midbrain dopaminergic neurons and protect them against MPP+ toxicity. The EMBO Journal, 14(4), 736-42.

Lang, A. E., Gill, S., Patel, N. K., Lozano, A., Nutt, J. G., Penn, R., ... Traub, M. (2006). Randomized controlled trial of intraputamenal glial cell line-derived neurotrophic factor infusion in Parkinson disease. Annals of Neurology, 59(3), 459-66.

Langston, J. W., Forno, L. S., Tetrud, J., \& Reeves, a G. (1999). Evidence of Active Nerve Cell Degeneration in the Substantia Nigra of Humans Years after 1-methyl-phenyl1,2,3,6-tetrahydrophyridine exposure. Annals of Neurology, 46, 598-605.

Lázaro, D. F., Rodrigues, E. F., Langohr, R., Shahpasandzadeh, H., Ribeiro, T., Guerreiro, P., ... Outeiro, T. F. (2014). Systematic Comparison of the Effects of Alpha-synuclein Mutations on Its Oligomerization and Aggregation. PLoS Genetics, 10(11), e1004741.

Le, W., Rowe, D., Xie, W., Ortiz, I., He, Y., \& Appel, S. H. (2001). Microglial activation and dopaminergic cell injury: an in vitro model relevant to Parkinson's disease. The Journal of Neuroscience: The Official Journal of the Society for Neuroscience, 21(21), 8447-8455.

Lee, R. C., Feinbaum, R. L., \& Ambros, V. (1993). The C. elegans heterochronic gene lin-4 encodes small RNAs with antisense complementarity to lin-14. Cell, 75(5), 843-854. 
Lees, A. J. (2007). Unresolved issues relating to the shaking palsy on the celebration of James Parkinson's 250th birthday. Movement Disorders: Official Journal of the Movement Disorder Society, 22 Suppl 1, S327-34.

Lees, A. J., Selikhova, M., Andrade, L. A., \& Duyckaerts, C. (2008). The black stuff and Konstantin Nikolaevich Tretiakoff. Movement Disorders, 23(6), 777-783.

Lewis, B. P., Burge, C. B., \& Bartel, D. P. (2005). Conserved seed pairing, often flanked by adenosines, indicates that thousands of human genes are microRNA targets. Cell, 120(1), 15-20.

Li, L., Chen, H., Chen, F., Li, F., Wang, M., Wang, L., ... Gao, D. (2013a). Effects of glial cell line-derived neurotrophic factor on microRNA expression in a 6-hydroxydopamineinjured dopaminergic cell line. Journal of Neural Transmission (Vienna, Austria: 1996), 120(11), 1511-23.

Li, L., Chen, H.-Z., Chen, F.-F., Li, F., Wang, M., Wang, L., ... Gao, D.-S. (2013b). Global microRNA expression profiling reveals differential expression of target genes in 6hydroxydopamine-injured MN9D cells. Neuromolecular Medicine, 15(3), 593-604.

Li, L.-H., Qin, H.-Z., Wang, J.-L., Wang, J., Wang, X.-L., \& Gao, G.-D. (2009). Axonal Degeneration of Nigra-striatum Dopaminergic Neurons Induced by 1-Methyl-4phenyl-1,2,3, 6-tetrahydropyridine in Mice. Journal of International Medical Research, 37(2), 455-463.

Li, X., Chen, C., Tu, Y., Sun, H., Zhao, M., Cheng, S., ... Zhang, S. (2013). Sirt1 Promotes Axonogenesis by Deacetylation of Akt and Inactivation of GSK3. Molecular Neurobiology, 48(3), 490-499.

Liberatore, G. T., Jackson-Lewis, V., Vukosavic, S., Mandir, A. S., Vila, M., McAuliffe, W. G., ... Przedborski, S. (1999). Inducible nitric oxide synthase stimulates dopaminergic neurodegeneration in the MPTP model of Parkinson disease. Nature Medicine, 5(12), 1403-9.

Lin, L., Doherty, D., Lile, J., Bektesh, S., \& Collins, F. (1993). GDNF: a glial cell line-derived neurotrophic factor for midbrain dopaminergic neurons. Science, 260(5111), 11301132.

Lin, M. T., \& Beal, M. F. (2006). Mitochondrial dysfunction and oxidative stress in neurodegenerative diseases. Nature, 443(7113), 787-795.

Liu, C.-M., Wang, R.-Y., Saijilafu, Jiao, Z.-X., Zhang, B.-Y., \& Zhou, F.-Q. (2013). MicroRNA138 and SIRT1 form a mutual negative feedback loop to regulate mammalian axon regeneration. Genes \& Development, 27(13), 1473-83. 
Liu, X. S., Chopp, M., Zhang, R. L., Tao, T., Wang, X. L., Kassis, H., ... Zhang, Z. G. (2011). MicroRNA profiling in subventricular zone after stroke: MiR-124a regulates proliferation of neural progenitor cells through Notch signaling pathway. PloS One, 6(8), e23461.

Lu, L., Neff, F., Alvarez-Fischer, D., Henze, C., Xie, Y., Oertel, W. H., ... Hartmann, A. (2005). Gene expression profiling of Lewy body-bearing neurons in Parkinson's disease. Experimental Neurology, 195(1), 27-39.

Luo, H., Wu, Q., Ye, X., Xiong, Y., Zhu, J., Xu, J., ... Wan, J. (2014). Genome-wide analysis of miRNA signature in the APPswe/PS1 1 E9 mouse model of alzheimer's disease. PloS One, 9(8), e101725.

Magill, S. T., Cambronne, X. A., Luikart, B. W., Lioy, D. T., Leighton, B. H., Westbrook, G. L., ... Goodman, R. H. (2010). microRNA-132 regulates dendritic growth and arborization of newborn neurons in the adult hippocampus. Proceedings of the National Academy of Sciences of the United States of America, 107(47), 20382-7.

Makeyev, E. V, Zhang, J., Carrasco, M. A., \& Maniatis, T. (2007). The MicroRNA miR-124 promotes neuronal differentiation by triggering brain-specific alternative pre-mRNA splicing. Molecular Cell, 27(3), 435-48.

Malagelada, C., Jin, Z. H., Jackson-Lewis, V., Przedborski, S., \& Greene, L. A. (2010). Rapamycin Protects against Neuron Death in In Vitro andln Vivo Models of Parkinson's Disease. Journal of Neuroscience, 30(3), 1166-1175.

Mann, V. M., Cooper, J. M., Daniel, S. E., Srai, K., Jenner, P., Marsden, C. D., \& Schapira, a. H. V. (1994). Complex I, iron, and ferritin in Parkinson's disease substantia nigra. Annals of Neurology, 36(6), 876-881.

McGeer, P. L., \& McGeer, E. G. (2008). Glial reactions in Parkinson's disease. Movement Disorders, 23(4), 474-483.

McKiernan, R. C., Jimenez-Mateos, E. M., Sano, T., Bray, I., Stallings, R. L., Simon, R. P., \& Henshall, D. C. (2012). Expression profiling the microRNA response to epileptic preconditioning identifies miR-184 as a modulator of seizure-induced neuronal death. Experimental Neurology, 237(2), 346-54.

McNaught, K. S. P., Mytilineou, C., Jnobaptiste, R., Yabut, J., Shashidharan, P., Jennert, P., \& Olanow, C. W. (2002). Impairment of the ubiquitin-proteasome system causes dopaminergic cell death and inclusion body formation in ventral mesencephalic cultures. Journal of Neurochemistry, 81(2), 301-6. 
McNaught, K. S. P., Perl, D. P., Brownell, A. L., \& Olanow, C. W. (2004). Systemic exposure to proteasome inhibitors causes a progressive model of Parkinson's disease. Annals of Neurology, 56(1), 149-162.

Mendes, N. D., Freitas, A. T., \& Sagot, M.-F. (2009). Current tools for the identification of miRNA genes and their targets. Nucleic Acids Research, 37(8), 2419-33.

Merchant, J. L., Du, M., \& Todisco, A. (1999). Sp1 phosphorylation by Erk 2 stimulates DNA binding. Biochemical and Biophysical Research Communications, 254(2), 45461.

Miñones-Moyano, E., Porta, S., Escaramís, G., Rabionet, R., Iraola, S., Kagerbauer, B., ... Martí, E. (2011). MicroRNA profiling of Parkinson's disease brains identifies early downregulation of miR-34b/c which modulate mitochondrial function. Human Molecular Genetics, 20(15), 3067-78.

Miska, E. A., Alvarez-Saavedra, E., Townsend, M., Yoshii, A., Sestan, N., Rakic, P., ... Horvitz, H. R. (2004). Microarray analysis of microRNA expression in the developing mammalian brain. Genome Biology, 5(9), R68.

Mitsumoto, Y., Watanabe, A., Mori, A., \& Koga, N. (1998). Spontaneous Regeneration of Nigrostriatal Dopaminergic Neurons in MPTP-Treated C57BL/6 Mice. Biochemical and Biophysical Research Communications, 248(3), 660-663.

Moore, D. J., Dawson, V. L., \& Dawson, T. M. (2003). Role for the ubiquitin-proteasome system in Parkinson's disease and other neurodegenerative brain amyloidoses. Neuromolecular Medicine, 4(1-2), 95-108.

Müller, M., Kuiperij, H. B., Claassen, J. A., Küsters, B., \& Verbeek, M. M. (2014). MicroRNAs in Alzheimer's disease: differential expression in hippocampus and cellfree cerebrospinal fluid. Neurobiology of Aging, 35(1), 152-8.

Nelson, P. T., \& Wang, W.-X. (2010). MiR-107 is reduced in Alzheimer's disease brain neocortex: validation study. Journal of Alzheimer's Disease : JAD, 21(1), 75-9.

Nielsen, J. A., Lau, P., Maric, D., Barker, J. L., \& Hudson, L. D. (2009). Integrating microRNA and mRNA expression profiles of neuronal progenitors to identify regulatory networks underlying the onset of cortical neurogenesis. BMC Neuroscience, 10(1), 98.

Nuytemans, K., Theuns, J., Cruts, M., \& Van Broeckhoven, C. (2010). Genetic etiology of Parkinson disease associated with mutations in the SNCA, PARK2, PINK1, PARK7, and LRRK2 genes: a mutation update. Human Mutation, 31(7), 763-780.

Obeso, J. A., Rodriquez-Oroz, M. C., Radriquez, M., Lanciego, J. L., Artieda, J., Gonzalo, N., \& Olanow, C. W. (2000). Pathophysiology of the basal ganglia in Parkinson's disease. 
Trends Neurosciences, 23(Box 1), 9-19.

Olde Loohuis, N. F. M., Kos, A., Martens, G. J. M., Van Bokhoven, H., Nadif Kasri, N., \& Aschrafi, A. (2012). MicroRNA networks direct neuronal development and plasticity. Cellular and Molecular Life Sciences : CMLS, 69(1), 89-102.

Packer, A. N., Xing, Y., Harper, S. Q., Jones, L., \& Davidson, B. L. (2008). The bifunctional microRNA miR-9/miR-9* regulates REST and COREST and is downregulated in Huntington's disease. The Journal of Neuroscience : The Official Journal of the Society for Neuroscience, 28(53), 14341-6.

Papadopoulos, G. L., Alexiou, P., Maragkakis, M., Reczko, M., \& Hatzigeorgiou, a G. (2009). DIANA-mirPath: Integrating human and mouse microRNAs in pathways. Bioinformatics (Oxford, England), 25(15), 1991-3.

Park, K. K., Liu, K., Hu, Y., Smith, P. D., Wang, C., Cai, B., ... He, Z. (2008). Promoting Axon Regeneration in the Adult CNS by Modulation of the PTEN/mTOR Pathway. Science, 322(5903), 963-966.

Parkkinen, L., O’Sullivan, S. S., Collins, C., Petrie, A., Holton, J. L., Revesz, T., \& Lees, A. J. (2011). Disentangling the relationship between lewy bodies and nigral neuronal loss in Parkinson's disease. Journal of Parkinson's Disease, 1(3), 277-86.

Patel, M., Cai, Q., Ding, D., Salvi, R., Hu, Z., \& Hu, B. H. (2013). The miR-183/Taok1 target pair is implicated in cochlear responses to acoustic trauma. PloS One, 8(3), e58471.

Patel, N. K., Bunnage, M., Plaha, P., Svendsen, C. N., Heywood, P., \& Gill, S. S. (2005). Intraputamenal infusion of glial cell line-derived neurotrophic factor in PD: a twoyear outcome study. Annals of Neurology, 57(2), 298-302.

Pathania, M., Torres-Reveron, J., Yan, L., Kimura, T., Lin, T. V, Gordon, V., ... Bordey, A. (2012). miR-132 enhances dendritic morphogenesis, spine density, synaptic integration, and survival of newborn olfactory bulb neurons. PloS One, 7(5), e38174.

Paxinos, G., \& Franklin, K. B. J. (2004). The Mouse Brain in Stereotaxic Coordinates (2nd ed.). Elsevier Science.

Pereira, J. B., Ibarretxe-Bilbao, N., Marti, M.-J., Compta, Y., Junqué, C., Bargallo, N., \& Tolosa, E. (2012). Assessment of cortical degeneration in patients with Parkinson's disease by voxel-based morphometry, cortical folding, and cortical thickness. Human Brain Mapping, 33(11), 2521-34.

Perrone-Capano, C., \& Porzio, U. D. I. (2000). Genetic and epigenetic control of midbrain dopaminergic neuron development. Int. J. Dev. Biol., 44, 679-687.

Piccini, P., Pavese, N., Hagell, P., Reimer, J., Björklund, A., Oertel, W. H., ... Lindvall, O. (2005). Factors affecting the clinical outcome after neural transplantation in 
Parkinson's disease. Brain : A Journal of Neurology, 128(Pt 12), 2977-86.

Pollock, A., Bian, S., Zhang, C., Chen, Z., \& Sun, T. (2014). Growth of the developing cerebral cortex is controlled by microRNA-7 through the p53 pathway. Cell Reports, 7(4), 1184-96.

Pong, K., Xu, R. Y., Baron, W. F., Louis, J.-C., \& Beck, K. D. (2002). Inhibition of Phosphatidylinositol 3-Kinase Activity Blocks Cellular Differentiation Mediated by Glial Cell Line-Derived Neurotrophic Factor in Dopaminergic Neurons. Journal of Neurochemistry, 71(5), 1912-1919.

Ponsen, M. M., Stoffers, D., Booij, J., van Eck-Smit, B. L. F., Wolters, E. C., \& Berendse, H. W. (2004). Idiopathic hyposmia as a preclinical sign of Parkinson's disease. Annals of Neurology, 56(2), 173-181.

Proukakis, C., Houlden, H., \& Schapira, A. H. (2013). Somatic alpha-synuclein mutations in Parkinson's disease: Hypothesis and preliminary data. Movement Disorders, 28(6), 705-712.

Przedborski, S., \& Jackson-Lewis, V. (1998). Experimental developments in movement disorders: update on proposed free radical mechanisms. Current Opinion in Neurology, 11(4), 335-339.

Przedborski, S., Jackson-Lewis, V., Naini, A. B., Jakowec, M., Petzinger, G., Miller, R., \& Akram, M. (2001). The parkinsonian toxin 1-methyl-4-phenyl-1,2,3,6tetrahydropyridine (MPTP): A technical review of its utility and safety. Journal of Neurochemistry.

REDMOND, D. (1986). FETAL NEURONAL GRAFTS IN MONKEYS GIVEN METHYLPHENYLTETRAHYDROPYRIDINE. The Lancet, 327(8490), 1125-1127.

Remenyi, J., Hunter, C. J., Cole, C., Ando, H., Impey, S., Monk, C. E., ... Arthur, J. S. C. (2010). Regulation of the miR-212/132 locus by MSK and CREB in response to neurotrophins. Biochemical Journal, 428, 281-291.

Saal, K.-A., Koch, J. C., Tatenhorst, L., Szegő, E. M., Ribas, V. T., Michel, U., ... Lingor, P. (2015). AAV.shRNA-mediated downregulation of ROCK2 attenuates degeneration of dopaminergic neurons in toxin-induced models of Parkinson's disease in vitro and in vivo. Neurobiology of Disease, 73, 150-62.

Saba, R., Goodman, C. D., Huzarewich, R. L. C. H., Robertson, C., \& Booth, S. A. (2008). A miRNA signature of prion induced neurodegeneration. PloS One, 3(11), e3652.

Satoh, J. (2012). Molecular network of microRNA targets in Alzheimer's disease brains. Experimental Neurology, 235(2), 436-46. 
Saunders, L. R., Sharma, A. D., Tawney, J., Nakagawa, M., Okita, K., Yamanaka, S., ... Verdin, E. (2010). miRNAs regulate SIRT1 expression during mouse embryonic stem cell differentiation and in adult mouse tissues. Aging, 2(7), 415-31.

Sawada, H., Ibi, M., Kihara, T., Urushitani, M., Nakanishi, M., Akaike, A., \& Shimohama, S. (2000). Neuroprotective Mechanism of Glial Cell Line-Derived Neurotrophic Factor in Mesencephalic Neurons. Journal of Neurochemistry, 74(3), 1175-1184.

Schaefer, A., O'Carroll, D., Tan, C. L., Hillman, D., Sugimori, M., Llinas, R., \& Greengard, P. (2007). Cerebellar neurodegeneration in the absence of microRNAs. The Journal of Experimental Medicine, 204(7), 1553-8.

Schallert, T., Fleming, S. M., Leasure, J. L., Tillerson, J. L., \& Bland, S. T. (2000). CNS plasticity and assessment of forelimb sensorimotor outcome in unilateral rat models of stroke, cortical ablation, parkinsonism and spinal cord injury. Neuropharmacology.

Schapira, A. H., \& Jenner, P. (2011). Etiology and pathogenesis of Parkinson's disease. Movement Disorders, 26(6), 1049-1055.

Schapira, A. H. V, Cooper, T. S. J. M., Clark, J. B., Jenner, P., \& Marsden, T. C. D. (1990). Mitochondria1 Complex I Deficiency in Parkinson's Disease. Journal of Neurochemistry, 54, 823-827.

Schober, A. (2004). Classic toxin-induced animal models of Parkinson's disease: 6-OHDA and MPTP. Cell and Tissue Research, 318(1), 215-24.

Schratt, G. M., Tuebing, F., Nigh, E. A., Kane, C. G., Sabatini, M. E., Kiebler, M., \& Greenberg, M. E. (2006). A brain-specific microRNA regulates dendritic spine development. Nature, 439(7074), 283-9.

Schubert, M., Brazil, D. P., Burks, D. J., Kushner, J. A., Ye, J., Flint, C. L., ... White, M. F. (2003). Insulin receptor substrate-2 deficiency impairs brain growth and promotes tau phosphorylation. The Journal of Neuroscience : The Official Journal of the Society for Neuroscience, 23(18), 7084-92.

Schwartz, M., Butovsky, O., Brück, W., \& Hanisch, U.-K. (2006). Microglial phenotype: is the commitment reversible? Trends in Neurosciences, 29(2), 68-74.

Segura, M. F., Hanniford, D., Menendez, S., Reavie, L., Zou, X., Alvarez-diaz, S., ... Hernando, E. (2009). Aberrant miR-182 expression promotes melanoma metastasis by repressing $\mathrm{FOXO} 3$ and microphthalmia-associated transcription factor. Proceedings of the National Academy of Sciences of the United States of America, 106(6), 1814-9.

Shi, Y., Zhao, X., Hsieh, J., Wichterle, H., Impey, S., Banerjee, S., ... Kosik, K. S. (2010). MicroRNA regulation of neural stem cells and neurogenesis. The Journal of 
Neuroscience : The Official Journal of the Society for Neuroscience, 30(45), 14931-6.

Shioya, M., Obayashi, S., Tabunoki, H., Arima, K., Saito, Y., Ishida, T., \& Satoh, J. (2010). Aberrant microRNA expression in the brains of neurodegenerative diseases: miR-29a decreased in Alzheimer disease brains targets neurone navigator 3. Neuropathology and Applied Neurobiology, 36(4), 320-30.

SIDHU, A., Wersinger, C., Moussa, C. E.-H., \& Vernier, P. (2004). The Role of aplhaSynuclein in Both Neuroprotection and Neurodegeneration. Annals of the New York Academy of Sciences, 1035(1), 250-270.

Simms, D., Cizdziel, P. E., \& Chomczynski, P. (1993). TRIzol: A new reagent for optimal single-step isolation of RNA. FOCUS, 15(4).

Skovronsky, D. M., Lee, V. M.-Y., \& Trojanowski, J. Q. (2006). NEURODEGENERATIVE DISEASES: New Concepts of Pathogenesis and Their Therapeutic Implications. Annual Review of Pathology: Mechanisms of Disease, 1(1), 151-170.

Slevin, J. T., Gerhardt, G. A., Smith, C. D., Gash, D. M., Kryscio, R., \& Young, B. (2005). Improvement of bilateral motor functions in patients with Parkinson disease through the unilateral intraputaminal infusion of glial cell line-derived neurotrophic factor. Journal of Neurosurgery, 102(2), 216-22.

Smith, Y., Wichmann, T., Factor, S. a, \& DeLong, M. R. (2012). Parkinson's Disease Therapeutics: New Developments and Challenges Since the Introduction of Levodopa. Neuropsychopharmacology, 37(1), 213-246.

SOMMER, W., CUI, X., ERDMANN, B., WIKLUND, L., BRICCA, G., HEILIG, M., \& FUXE, K. (1998). The Spread and Uptake Pattern of Intracerebrally Administered Oligonucleotides in Nerve and Glial Cell Populations of the Rat Brain. Antisense and Nucleic Acid Drug Development, 8(2), 75-85.

Sosanya, N. M., Huang, P. P. C., Cacheaux, L. P., Chen, C. J., Nguyen, K., Perrone-Bizzozero, N. I., \& Raab-Graham, K. F. (2013). Degradation of high affinity HuD targets releases Kv1.1 mRNA from miR-129 repression by mTORC1. The Journal of Cell Biology, 202(1), 53-69.

Spillantini, G. M., Schmidt, M. L., Lee, V. M.-Y., Trojanowski, J. Q., Jakes, R., \& Goedert, M. (1997). Alpha-Synuclein in Lewy bodies. Nature, 839-840.

Srinivasan, S., Anitha, M., Mwangi, S., \& Heuckeroth, R. O. (2005). Enteric neuroblasts require the phosphatidylinositol 3-kinase/Akt/Forkhead pathway for GDNFstimulated survival. Molecular and Cellular Neurosciences, 29(1), 107-19.

St. P. McNaught, K., Belizaire, R., Isacson, O., Jenner, P., \& Olanow, C. W. (2003). Altered Proteasomal Function in Sporadic Parkinson's Disease. Experimental Neurology, 
179(1), 38-46.

Stamboulian, S., Choi, J.-S., Ahn, H.-S., Chang, Y.-W., Tyrrell, L., Black, J. A., ... Dib-Hajj, S. D. (2010). ERK1/2 mitogen-activated protein kinase phosphorylates sodium channel $\mathrm{Na}(\mathrm{v}) 1.7$ and alters its gating properties. The Journal of Neuroscience: The Official Journal of the Society for Neuroscience, 30(5), 1637-47.

Stanic, D., Finkelstein, D. I., Bourke, D. W., Drago, J., \& Horne, M. K. (2003). Timecourse of striatal re-innervation following lesions of dopaminergic SNpc neurons of the rat. European Journal of Neuroscience, 18(5), 1175-1188.

Stefani, G., \& Slack, F. J. (2008). Small non-coding RNAs in animal development. Nat Rev Mol Cell Biol, 9(3), 219-230.

Stevanato, L., \& Sinden, J. D. (2014). The effects of microRNAs on human neural stem cell differentiation in two- and three-dimensional cultures. Stem Cell Research \& Therapy, 5(2), 49.

Sullivan, A. M., \& Toulouse, A. (2011). Neurotrophic factors for the treatment of Parkinson's disease. Cytokine \& Growth Factor Reviews, 22(3), 157-65.

Sundberg, M., Bogetofte, H., Lawson, T., Jansson, J., Smith, G., Astradsson, A., ... Isacson, O. (2013). Improved cell therapy protocols for Parkinson's disease based on differentiation efficiency and safety of hESC-, hiPSC-, and non-human primate iPSCderived dopaminergic neurons. Stem Cells (Dayton, Ohio), 31(8), 1548-62.

Takagi, Y., Takahashi, J., Saiki, H., Morizane, A., Hayashi, T., Kishi, Y., ... Hashimoto, N. (2005). Dopaminergic neurons generated from monkey embryonic stem cells function in a Parkinson primate model. The Journal of Clinical Investigation, 115(1), 102-9.

Tang, H., Bian, Y., Tu, C., Wang, Z., Yu, Z., Liu, Q., ... Li, G. (2015). The miR-183/96/182 Cluster Regulates Oxidative Apoptosis and Sensitizes Cells to Chemotherapy in Gliomas. Cell Biochemistry and Biophysics, 71(2), 649-659.

Tatarewicz, S. M., Wei, X., Gupta, S., Masterman, D., Swanson, S. J., \& Moxness, M. S. (2007). Development of a maturing T-cell-mediated immune response in patients with idiopathic Parkinson's disease receiving $r$-metHuGDNF via continuous intraputaminal infusion. Journal of Clinical Immunology, 27(6), 620-7.

Tavares, I. A., Touma, D., Lynham, S., Troakes, C., Schober, M., Causevic, M., ... Morris, J. D. H. (2013). Prostate-derived Sterile 20-like Kinases (PSKs/TAOKs) Phosphorylate Tau Protein and Are Activated in Tangle-bearing Neurons in Alzheimer Disease. Journal of Biological Chemistry, 288(21), 15418-15429. 
Terasawa, K., Ichimura, A., Sato, F., Shimizu, K., \& Tsujimoto, G. (2009). Sustained activation of ERK1/2 by NGF induces microRNA-221 and 222 in PC12 cells. The FEBS Journal, 276(12), 3269-76.

Tillerson, J. ., Caudle, W. ., Reverón, M. ., \& Miller, G. . (2003). Exercise induces behavioral recovery and attenuates neurochemical deficits in rodent models of Parkinson's disease. Neuroscience, 119(3), 899-911.

Tillerson, J. L., Cohen, A. D., Caudle, W. M., Zigmond, M. J., Schallert, T., \& Miller, G. W. (2002). Forced nonuse in unilateral parkinsonian rats exacerbates injury. The Journal of Neuroscience : The Official Journal of the Society for Neuroscience, 22(15), 67906799.

Tomac, A., Lindqvist, E., Lin, L. F., Ogren, S. O., Young, D., Hoffer, B. J., \& Olson, L. (1995). Protection and repair of the nigrostriatal dopaminergic system by GDNF in vivo. Nature, 373(6512), 335-9.

Tompkins, M. M., \& Hill, W. D. (1997). Contribution of somal Lewy bodies to neuronal death. Brain Research, 775(1-2), 24-29.

Tönges, L., Frank, T., Tatenhorst, L., Saal, K. A., Koch, J. C., Szego, É. M., ... Lingor, P. (2012). Inhibition of rho kinase enhances survival of dopaminergic neurons and attenuates axonal loss in a mouse model of Parkinson's disease. Brain.

Torres, A., Torres, K., Pesci, A., Ceccaroni, M., Paszkowski, T., Cassandrini, P., ... Maciejewski, R. (2012). Deregulation of miR-100, miR-99a and miR-199b in tissues and plasma coexists with increased expression of mTOR kinase in endometrioid endometrial carcinoma. BMC Cancer, 12(1), 369.

Ugarte, S. D., Lin, E., Klann, E., Zigmond, M. J., \& Perez, R. G. (2003). Effects of GDNF on 6OHDA-induced death in a dopaminergic cell line: modulation by inhibitors of PI3 kinase and MEK. Journal of Neuroscience Research, 73(1), 105-12.

Urdinguio, R. G., Fernandez, A. F., Lopez-Nieva, P., Rossi, S., Huertas, D., Kulis, M., ... Esteller, M. (2010). Disrupted microRNA expression caused by Mecp2 loss in a mouse model of Rett syndrome. Epigenetics, 5(7), 656-63.

van Rooij, E., \& Kauppinen, S. (2014). Development of microRNA therapeutics is coming of age. EMBO Molecular Medicine, 6(7), 851-864.

van Rooij, E., Purcell, A. L., \& Levin, A. A. (2012). Developing microRNA therapeutics. Circulation Research, 110(3), 496-507.

van Spronsen, M., van Battum, E. Y., Kuijpers, M., Vangoor, V. R., Rietman, M. L., Pothof, J., ... Hoogenraad, C. C. (2013). Developmental and activity-dependent miRNA expression profiling in primary hippocampal neuron cultures. PloS One, 8(10), 
e74907.

Visvanathan, J., Lee, S., Lee, B., Lee, J. W., \& Lee, S.-K. (2007). The microRNA miR-124 antagonizes the anti-neural REST/SCP1 pathway during embryonic CNS development. Genes \& Development, 21(7), 744-9.

Vo, N., Klein, M. E., Varlamova, O., David, M., Yamamoto, T., \& Goodman, R. H. (2006). A cAMP-response element binding protein-induced microRNA regulates neuronal morphogenesis. Proc . Natl . Acad . Sci . USA, 103(3), 16426-16431.

Wang, G., van der Walt, J. M., Mayhew, G., Li, Y.-J., Züchner, S., Scott, W. K., ... Vance, J. M. (2008). Variation in the miRNA-433 binding site of FGF20 confers risk for Parkinson disease by overexpression of alpha-synuclein. American Journal of Human Genetics, 82(2), 283-9.

Wang, H., Duan, X., Ren, Y., Liu, Y., Huang, M., Liu, P., ... Zheng, W. (2013). FoxO3a negatively regulates nerve growth factor-induced neuronal differentiation through inhibiting the expression of neurochondrin in PC12 cells. Molecular Neurobiology, 47(1), 24-36.

Wang, W.-X., Rajeev, B. W., Stromberg, A. J., Ren, N., Tang, G., Huang, Q., ... Nelson, P. T. (2008). The expression of microRNA miR-107 decreases early in Alzheimer's disease and may accelerate disease progression through regulation of beta-site amyloid precursor protein-cleaving enzyme 1. The Journal of Neuroscience: The Official Journal of the Society for Neuroscience, 28(5), 1213-23.

Wightman, B., Ha, I., \& Ruvkun, G. (1993). Posttranscriptional regulation of the heterochronic gene lin-14 by lin-4 mediates temporal pattern formation in $\mathrm{C}$. elegans. Cell, 75(5), 855-862.

Williams, A. H., Valdez, G., Moresi, V., Qi, X., McAnally, J., Elliott, J. L., ... Olson, E. N. (2009). MicroRNA-206 delays ALS progression and promotes regeneration of neuromuscular synapses in mice. Science (New York, N.Y.), 326(5959), 1549-54.

Wu, D. C., Jackson-Lewis, V., Vila, M., Tieu, K., Teismann, P., Vadseth, C., ... Przedborski, S. (2002). Blockade of microglial activation is neuroprotective in the 1-methyl-4-phenyl1,2,3,6-tetrahydropyridine mouse model of Parkinson disease. The Journal of Neuroscience : The Official Journal of the Society for Neuroscience, 22(5), 1763-1771.

Wu, J., Qian, J., Li, C., Kwok, L., Cheng, F., Liu, P., ... Lü, J. (2014). miR-129 regulates cell proliferation by downregulating Cdk6 expression. Cell Cycle, 9(9), 1809-1818.

Yang, F., Feng, L., Zheng, F., Johnson, S. W., Du, J., Shen, L., ... Lu, B. (2001). GDNF acutely modulates excitability and A-type $\mathrm{K}(+)$ channels in midbrain dopaminergic neurons. Nature Neuroscience, 4(11), 1071-8. 
Yang, J.-S., \& Lai, E. C. (2011). Alternative miRNA biogenesis pathways and the interpretation of core miRNA pathway mutants. Molecular Cell, 43(6), 892-903.

Yang, W.-B., Chen, P.-H., Hsu, T., Fu, T.-F., Su, W.-C., Liaw, H., ... Hung, J.-J. (2014). Sp1mediated microRNA-182 expression regulates lung cancer progression. Oncotarget, 5(3), 740-53.

Yeom, K.-H., Lee, Y., Han, J., Suh, M. R., \& Kim, V. N. (2006). Characterization of DGCR8/Pasha, the essential cofactor for Drosha in primary miRNA processing. Nucleic Acids Research, 34(16), 4622-9.

Yu, H., Wu, M., Zhao, P., Huang, Y., Wang, W., \& Yin, W. (2015). Neuroprotective effects of viral overexpression of microRNA-22 in rat and cell models of cerebral ischemiareperfusion injury. Journal of Cellular Biochemistry, 116(2), 233-41.

Yuan, Z., Lehtinen, M. K., Merlo, P., Villén, J., Gygi, S., \& Bonni, A. (2009). Regulation of neuronal cell death by MST1-FOXO1 signaling. The Journal of Biological Chemistry, 284(17), 11285-92.

Zhong, Z., Wen, Z., \& Darnell, J. E. (1994). Stat3: a STAT family member activated by tyrosine phosphorylation in response to epidermal growth factor and interleukin-6. Science (New York, N.Y.), 264(5155), 95-8.

Zhou, S., Shen, D., Wang, Y., Gong, L., Tang, X., Yu, B., ... Ding, F. (2012). microRNA-222 targeting PTEN promotes neurite outgrowth from adult dorsal root ganglion neurons following sciatic nerve transection. PloS One, 7(9), e44768.

Zovoilis, A., Agbemenyah, H. Y., Agis-Balboa, R. C., Stilling, R. M., Edbauer, D., Rao, P., ... Fischer, A. (2011). microRNA-34c is a novel target to treat dementias. The EMBO Journal, 30(20), 4299-308. 


\section{Acknowledgements}

First, I want to thank Prof. Dr. Paul Lingor for the opportunity to conduct my PhD thesis in his laboratory. I am thankful for his support and guidance but especially for the freedom I had during this time to work independently. I learned a lot.

Furthermore, I thank Prof. Dr. Mathias Bähr for giving me the chance to work in his department.

I also want to thank Prof. Dr. André Fischer for being part of my thesis committee, the great cooperation and the helpful suggestions and discussions. Additionally, I want to express my gratitude to Prof. Dr. Tiago Outerio for being part my thesis committee and the inspiring discussions. Furthermore, I want to thank the members of my extended examination board Prof. Dr. Silvio Rizzoli, Prof. Dr. Michael Hörner and Dr. Sebastian Kügler for agreeing to evaluate my work and attend my PhD defense.

Special thanks to Rashi Halder, my contact person in the Fischer lab, for taking care of the sequencing experiments and for answering so many questions.

The GGNB and Neuroscience office: Thanks to all the people who take care of us PhD students there, especially Michael for his support in organizational issues and making so much possible.

My deepest gratitude goes to the AG Lingor, especially to Vivian Dambeck and Lisa Barski for their excellent technical support and the helpful discussions. Also a special thanks goes to Lars Tatenhorst for sharing so many hours of stereotactical surgery with me, I do not know how I should have done this without your help. Kimmi, THANK YOU for sharing craziness and everything else. Additionally, I want to say thank you to my Bachelor student Sören Noack for all the blotting and his curiosity. Great job! And of course all the others, you crazy bunch of people, thank you for the great atmosphere in our lab...

Of course I also thank all other members of the Waldweg Labs for answering questions, technical help and the nice time...

Natürlich danke ich meiner Familie. Mama, Papa, Steffen, Großmütterchen, Kathi, aber natürlich auch Iris und Thomas. Aus tiefstem Herzen Danke für Euren Zuspruch, Eure Unterstützung und für's Dasein. Und dafür, dass Ihr manchmal mehr an mich geglaubt habt, als ich selbst.

Und zu guter Letzt geht ein riesengroßes Dankeschön an all meine Freunde und besonders an alle PhD-Leidensgenossen unter euch. Für's „Ins-Gedächtnis-rufen“, dass es auch andere Dinge im Leben gibt, für unzählige gute Gespräche und durchtanzte Nächte, Ermutigung, Ablenkung und für's Pläne schmieden. 


\section{Curriculum Vitae}

\section{Personal details}

Name

Adress

Date and place of birth

Nationality

\section{Education}

09/2012-04/2016

$10 / 2010-09 / 2012$

$04 / 2008-09 / 2010$

$10 / 2006-09 / 2010$

$04 / 2005-09 / 2006$

$10 / 2004-03 / 2005$

June 2003
Anna-Elisa Roser

Zeppelinstr. 7a

37083 Göttingen

$23^{\text {rd }}$ of April 1984 in Langenhagen

German

PhD Thesis Georg-August-University Göttingen

University Medicine, Department of Neurology

GGNB Program "Molecular Physiology of the Brain"

"miRNAs in protection and regeneration of dopaminergic

midbrain neurons" Supervisor: Prof. Dr. Paul Lingor

Master of Science Georg-August-University Göttingen

M.Sc. Program "Developmental, Neural and Behavioral

Biology"

Master Thesis "Astrocytic influence on microglial TLR

responses" Supervisor: Prof. Dr. Uwe-Karsten Hanisch

University Medicine, Department of Neuropathology

Bachelor of Science Leibniz-University Hannover

B.Sc. Program "Biology"

Bachelor Thesis "Prosodic cues in isolation calls of kitten" Supervisor: Prof. Dr. Elke Zimmermann

University of Veterinary Sciences Hannover, Department of Zoology

Bachelor of Science Leibniz-University Hannover

B.Sc. Program "Biology/German literature and linguistics" Bachelor Thesis "Prosodic cues in isolation calls of kitten" Supervisor: Prof. Dr. Elke Zimmermann

University of Veterinary Sciences Hannover, Department of Zoology

Vocational Training Nursing Practice

Henriettenstiftung Hannover

Dentistry Program

Hannover Medical School

Abitur Integrierte Gesamtschule Roderbruch Hannover 